\title{
Selectivity Enhancement of Gas Sensitive Field Effect Transistors by Dynamic Operation
}

\author{
Christian Bur
}

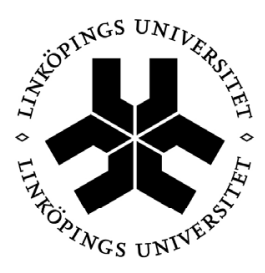

\section{Linköping University INSTITUTE OF TECHNOLOGY}

\author{
Division of Applied Sensor Science \\ Department of Physics, Chemistry, and Biology \\ Linköping University \\ SE-581 83 Linköping, Sweden
}

Linköping 2015

This thesis is the result of a joint $\mathrm{PhD}$ project together with the Lab for Measurement Technology, Saarland University, D-66123 Saarbrücken, Germany. Christian Bur was enrolled in The Joint European Doctoral Programme in Materials Science and Engineering - DocMASE. 
The cover image shows the signal of a dynamically operated SiC-FET gas sensor when both, the operating temperature and the applied gate bias are modulated. Additionally, chemical formulas of typical air pollutants, i.e., naphthalene, benzene, formaldehyde, and nitrogen oxides, are drawn as well as a two-dimensional scatter plot showing the discrimination of three classes.

(c) 2015 Christian Bur

ISBN: 978-91-7519-119-5

ISSN: 0345-7524

Printed by LiU-Tryck

SE-581 83 Linköping, Sweden 
To my family

without whom none of my success would be possible

in loving memory of my grandfathers

Albert Bretz

Bernhard Bur 



\section{Abstract}

Gas sensitive field effect transistors based on silicon carbide, SiC-FETs, have been applied to various applications mainly in the area of exhaust and combustion monitoring. So far, these sensors have normally been operated at constant temperatures and adaptations to specific applications have been done by material and transducer platform optimization.

In this thesis, the methodology of dynamic operation for selectivity enhancement is systematically developed for SiC-FETs. Temperature cycling, which is well known for metal oxide gas sensors, is transferred to SiC-FETs. Additionally, gate bias modulation is introduced increasing the performance further.

The multi-dimensional sensor data are evaluated by use of pattern recognition mainly based on multivariate statistics. Different strategies for feature selection, crossvalidation, and classification methods are studied.

After developing the methodology of dynamic operation, i.e., applying the virtual multi-sensor approach on SiC-FETs, the concept is validated by two different case studies under laboratory conditions: Discrimination of typical exhaust gases and quantification of nitrogen oxides in a varying background is presented. Additionally, discrimination and quantification of volatile organic compounds in the low parts-perbillion range for indoor air quality applications is demonstrated. The selectivity of SiC-FETs is enhanced further by combining temperature and gate bias cycled operation. Stability is increased by extended training. 



\section{Sammanfattning}

Gaskänsliga fält-effekt-transistorer baserade på halvledarmaterialet kiselkarbid (SiC-FET) har redan framgångsrikt använts för olika tillämpningar främst inom området för avgas- och förbränningsövervakning. Normalt har dessa sensorer använts vid konstant temperatur och anpassning till specifika tillämpningar har gjorts av material och sensor optimering.

I denna avhandling har metoden för dynamisk modulering systematiskt utvecklats för att ökaselektiviteten av SiC-FETs. Temperatur-cykling är en välkänd metod för metalloxidsensorer och har nu tillämpats på SiC-FETs för första gången. Likaså har den pålagda gatepotentialen varierats.

Mönsterigenkänningsmetoder baserade på multivariat statistik används för att utvärdera multi-dimensionella sensordata. Olika strategier för urval, korsvalidering och klassificering av okända uppgifter studeras.

Efter att metodiken för dynamiska mätmetoder har beskrivits i detalj, verifieras strategin av virtuella-multisensorer genom två tester under laboratorieförhållanden. Detta visas av exemplet med separering av typiska avgaser och bestämning av koncentrationen av kväveoxider i varierande gasblandningar. Vidare har ett test genomförts där flyktiga organiska föreningar identifieras och kvantifieras för att bestämma kvaliteten på inomhusluft. Dessutom kan man öka selektiviteten av sensorerna genom att kombinera modulering av temperatur och gate-potential. 



\section{Zusammenfassung}

Gassensitive Feldeffekt-Transistoren basierend auf Siliziumkarbid (SiC-FET) werden überwiegend für die Abgasmessung eingesetzt. Üblicherweise werden diese Sensoren bei konstanter Temperatur betrieben. Durch die Auswahl geeigneter Materialien sowie durch die Modifikation der Sensoren können diese für verschiedene Anwendungen optimiert werden.

In der vorliegenden Dissertation wird die Methodik einer dynamischen Betriebsweise zur Selektivitätssteigerung systematisch weiterentwickelt. Temperaturmodulation ist ein bewährtes Verfahren für Halbleitergassensoren, das hier auf SiC-FETs übertragen wird. In ähnlicher Weise wird auch das Gate-Potential zyklisch variiert.

Mustererkennungsverfahren basierend auf multivariater Statistik werden eingesetzt, um die mehrdimensionalen Messdaten auszuwerten. Verschiedene Verfahren zur Merkmalsauswahl, Kreuzvalidierung und Klassifikation unbekannter Daten werden untersucht.

Nachdem die Methodik ausführlich dargelegt wurde, wird der Ansatz des virtuellen Multisensors anhand zweier Anwendungen unter Laborbedingungen verifiziert. Dies wird am Beispiel der Konzentrationsbestimmung von Stickoxiden in variierenden Gasgemischen gezeigt. Zudem werden flüchtige organische Verbindungen im niedrigen ppb-Bereich zur Bestimmung der Innenraumluftqualität erkannt und quantifiziert. Die Selektivität kann durch die Kombination von Temperatur- und Potentialmodulation weiter gesteigert und Drifteinflüsse durch erweitertes Training kompensiert werden. 



\section{Populärvetenskaplig}

\section{Sammanfattning}

\section{Ökad prestanda hos gaskänsliga fälteffekttransistorer}

genom dynamisk drivning

Luftföroreningar är ett stort problem i dagens samhälle. Miljöfarliga emissioner, så kallade växthusgaser, bidrar uppenbarligen till global uppvärmning vilket leder till allvarliga klimatförändringar. Tung industri och trafik är de största källorna till dessa emissioner. Förutom koldioxid, $\mathrm{CO}_{2}$, vilket är en välkänd växthusgas, bidrar också kväveoxider, $\mathrm{NO}_{\mathrm{x}}$, väsentligt till global uppvärmning och negativ påverkan på vår miljö. Till exempel bildar $\mathrm{NO}_{\mathrm{x}}$, som finns i atmosfären, surt regn. Kyoto protokollet 1997 innebar att 37 industrinationer ingick avtal om att reducera sina totala emissioner. Som ett resultat av detta har allt mer strikta lagkrav för emissioner utfärdats. För att minska utsläppen t.ex från bilar, behövs gassensorer som kan mäta föroreningarna $\mathrm{i}$ bilavgaser för att systemet för efterbehandling av avgaser (katalysator osv) ska kunna fungera tillfredställande.

Tillsammans med luftföroreningarna utomhus är också kvalitén på inomhusluft ett stort problem. Vi människor tillbringar $80 \%$ av vår tid inomhus med mycket begränsad luftväxling. Brist på frisk luft kan leda till sjukahus-syndromet med akuta obehagssymtom som huvudvärk, irriterade slemhinnor, yrsel och koncentrationssvårigheter. Dessutom rapporteras ännu allvarligare hälsoproblem som cancer.

Reglering av dagens ventilationssystem är inte baserat på kvalitén på luften utan luften byts med vissa tidsmellanrum, vilket givetvis inte är optimalt för luftkvalitén. Dessa system är inte heller energioptimerade, de konsumerar upp till hälften av den totala energin som går åt till en byggnad. Därför är behovsstyrd ventilation mycket 
angeläget där man reglerar på kvalitén på inomhusluften. Sådana kontrollsystem kräver billiga, mycket känsliga och selektiva sensorer. De mest kritiska föroreningarna inomhus är flyktiga organiska föreningar som formaldehyd, bensen och naftalen. De är alla farliga för människor redan vid väldigt små koncentrationer och detektionen av dem är därför en stor utmaning.

Kemiska gassensorer är oftast lämpade för massproduktion och därför billiga. En berömd representant för kemiska gassensorer är den gaskänsliga fälteffekttransistorn baserad på kiselkarbid som det halvledande bärarmaterialet, SiC-FET. Kiselkarbiden gör det möjligt att använda sensorn vid hög temperatur och i korrosiv miljö t.ex. placerad direkt i avgaser. Emellertid finns det en nackdel med dessa sensorer, de är inte selektiva, dvs. de detekterar ett stort antal gaser men är inte särskilt specifika. Detta problem kan lösas genom att man använder en array av sensorer som tillsammans ger ett unikt fingeravtryck av gaserna man mäter på. Dock ökar kostnaden och komplexiteten i systemet avsevärt för varje ny sensor man lägger till arrayen. Ett alternativ är att använda bara en sensor som körs på ett dynamiskt sätt. Parametrar som sensortemperaturen kan varieras över en viss cykel vilket resulterar i ett liknande fingeravtryck som när man använder en sensor array. Detta kallas att man använder en virtuell sensorarray.

I denna avhandling, har för första gången detta virtuella multi-sensor koncept studerats systematiskt med en SiC-FET. Förutom arbetstemperaturen hos sensorn kan också andra sensorparametrar varieras, såsom pålagd spänning över sensorn, vilket ökar selektiviteten ytterligare. Det har visats att SiC-FET gas sensorer som körs på ett dynamiskt sätt kan detektera $\mathrm{NO}_{\mathrm{x}}$ koncentrationen $\mathrm{i}$ en varierande blandning av andra gaser vid mätningar i laboratoriemiljö. Metoden som har utvecklats i den här avhandlingen kan i framtiden användas för att förbättra efterbehandlingen av avgaser och därigenom reducera emissionerna från trafiken.

I nästa studie, visar avhandlingen att SiC-FET sensorer visar en tillräckligt hög respons till mycket låga koncentrationer ( $\mathrm{ppb}=$ parts per biljon) av giftiga flyktiga kolväteföroreningar i inomhusluft, t.ex. formaldehyd, bensen och naftalen. Preliminära resultat indikerar att dessa sensorer klarar att särskilja mellan dessa olika kolväten, vilket behövs för att bestämma kvalitén på inomhusluft. Ett dynamiskt arbetssätt ger emellertid stora datamängder och behov av smart datautvärdering. I denna avhandling har mönsterigenkänningsmetoder använts, vilka på ett effektivt sätt 
söker efter karakteristiska mönster i sensorsignalen, vilket sedan används för att identifiera föroreningen i fråga. Det är inte enbart algoritmerna som behandlas i avhandlingen, utan också metoder för att anpassa och optimera dem till ett givet problem. Resultaten i detta doktorandprojekt ger en starkt utökad verktygslåda av utvärderingsalgoritmer och strategier, vilka också kan användas i andra applikationer och projekt.

Till exempel har en utveckling av metoden använts för att mäta svaveldioxid, $\mathrm{SO}_{2}$, i en avsvavlingsanläggning i ett värmekraftverk i Växjö.

Slutsatsen blir att med det föreslagna dynamiska arbetssättet för SiC-FET sensorerna kan selektiviteten ökas väsentligen. Därför är SiC-FET gassensorerna lämpliga kandidater för emissionskontroll och applikationer för kontroll av kvalitén på inomhusluft. Dessa sensorer kan i kombination med relevanta kontrollsystem hjälpa till att öka effektiviteten på ventilationssystem i byggnader och därigenom reducera energikonsumtionen. 



\section{Preface}

This dissertation is the result of my joint $\mathrm{PhD}$ studies through The Joint European Doctoral Programme in Materials Science and Engineering - DocMASE of The European School of Materials - EUSMAT between May 2011 and February 2015. The work outlined in this thesis was carried out under the supervision of Prof. Dr. Andreas Schütze, Lab for Measurement Technology, Dept. of Physics and Mechatronic Engineering, Saarland University, Germany, Prof. Dr. Anita Lloyd Spetz, and Dr. Mike Andersson, both Div. of Applied Sensor Science, Dept. of Physics, Chemistry, and Biology (IFM), Linköping University, Sweden.

Research results obtained during my studies have been presented at international conferences and were published in peer-reviewed scientific journals. The dissertation itself is written as a monograph, however, a list of own publications and my contribution to these can be found at the end of the thesis. Permissions of already published figures and tables have been obtained from the publisher and a reference to the original research paper is given in each case.

The thesis follows a typical five-step structure with an introduction and motivation, in Chapters 1 and 2, a review of basics and the state of the art in Chapters 3-5, a description of the experimental setup in Chapter 6, the discussion of the developed methodology and obtained results, cf. Chapters 7 and 8 , and a summary of the work together with an outlook in the last chapter, cf. Chapter 9.

The structure in detail is as follows: After promoting the work in Chapter 1, an introduction to air pollutants in Chapter 2 is given. Theoretical basics of field effect based gas sensors and the state of the art sensor operation are reviewed in Chapters 3 and 4, respectively, and relevant references are given. The theory behind the applied signal processing as well as the used algorithms is presented in Chapter 5. My measurement setup and studied sensors are described in Chapter 6. Chapter 7 deals with the measurement methodology developed in this $\mathrm{PhD}$ project and adapted signal 
processing. These methods are then exemplarily applied to two different scenarios, exhaust monitoring, and indoor air quality. The obtained results are presented and discussed in Chapter 8 . The last section in Chapter 8 focusses on advanced dynamic operation and further considerations regarding signal processing. The work is summarized and an outlook is given in Chapter 9, followed by a list of all references. In the appendix, complete lists of own publications, used abbreviations and symbols are provided as well as list of all tables and figures shown in the thesis.

All this work and the doctorate itself would not have been possible without the support and help of my supervisors, colleagues, friends, and my family. I acknowledge these persons in a dedicated section.

Christian Bur

(February 2015) 


\section{Table of Contents}

1 INTRODUCTION .......................................................................................................

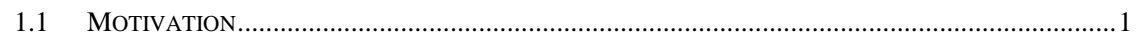

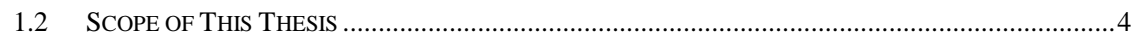

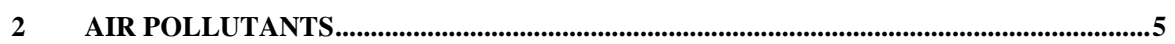

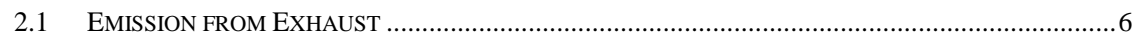

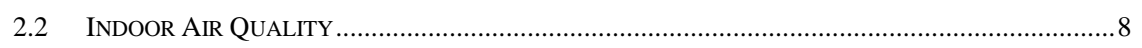

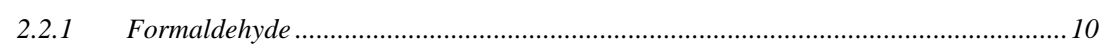

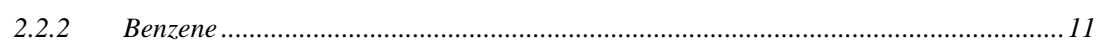

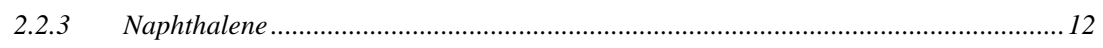

3 FIELD EFFECT BASED GAS SENSING …….......................................................................15

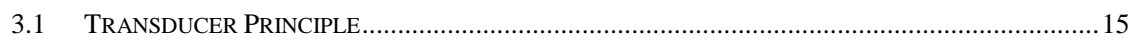

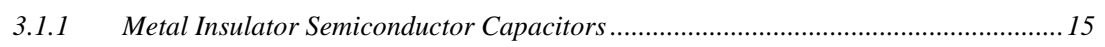

3.1.2 Metal Insulator Semiconductor Field Effect Transistors .......................................24

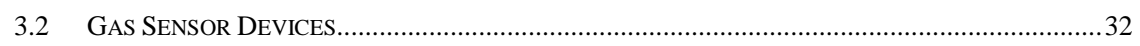

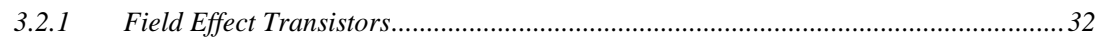

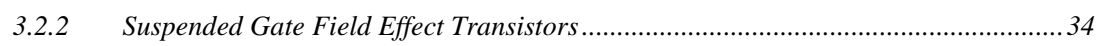

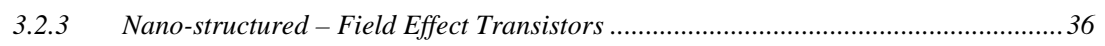

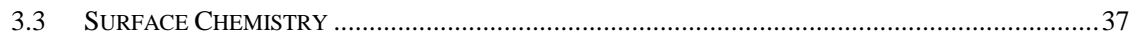

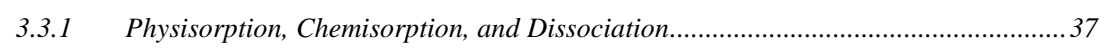

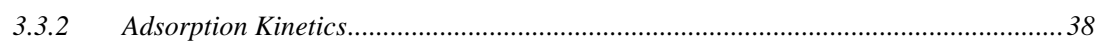

3.3.3 Workfunction Change by Adsorbates.................................................................... 40

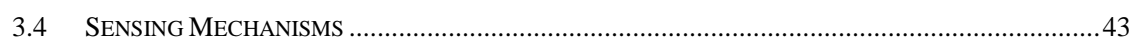

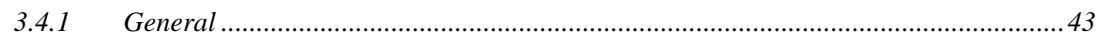

3.4.2 Hydrogen-Containing Gases............................................................................. 43

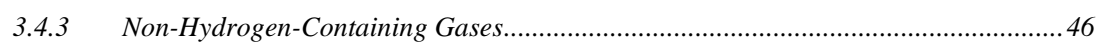

3.5 TERMINOLOGY RELATED TO GAS SENSORS …………................................................ 48

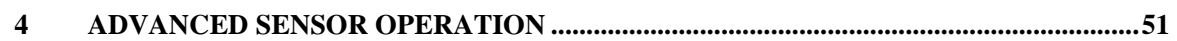

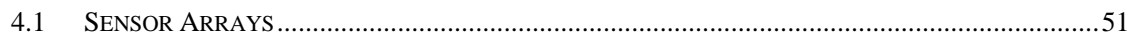

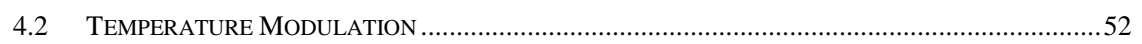




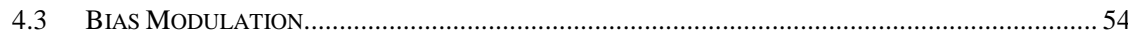

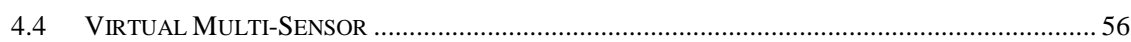

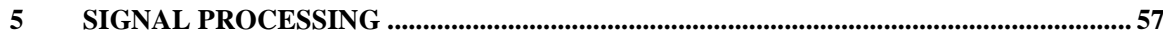

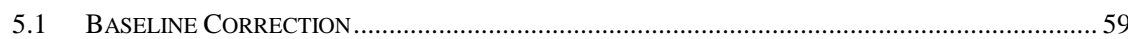

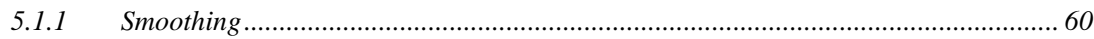

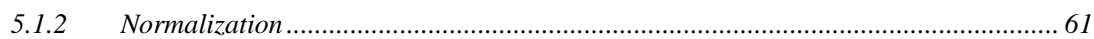

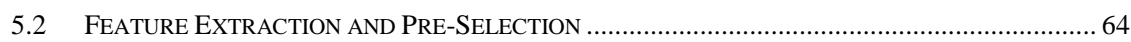

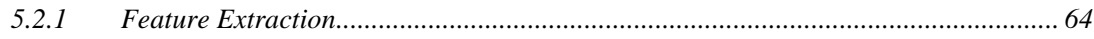

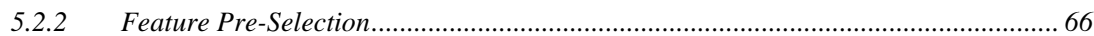

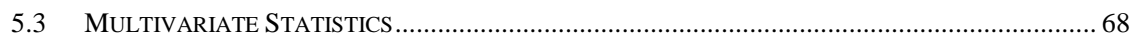

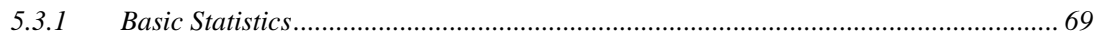

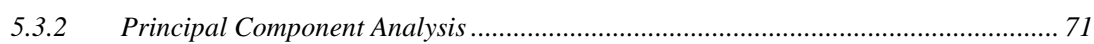

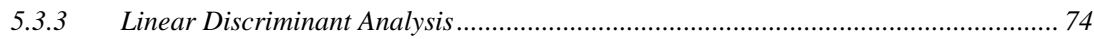

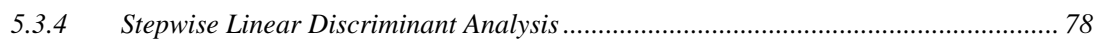

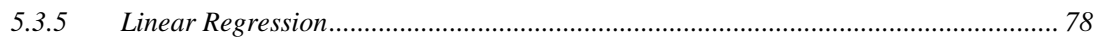

5.3.6 Partial Least Squares - Discriminant Analysis ................................................. 80

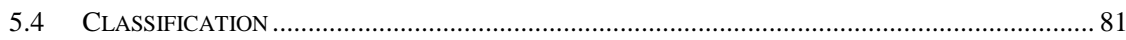

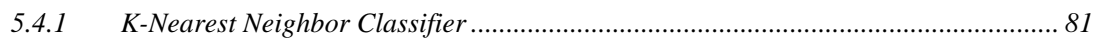

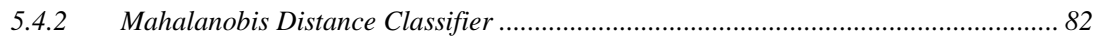

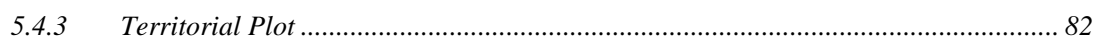

5.4.4 Maximum Likelihood and Bayes-Theorem ........................................................ 83

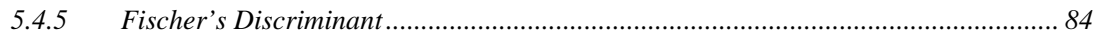

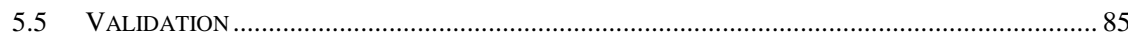

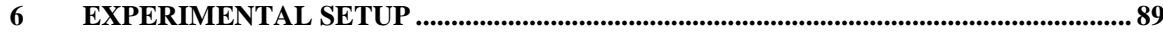

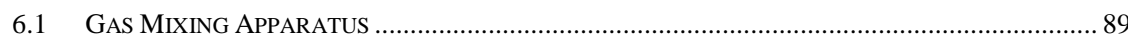

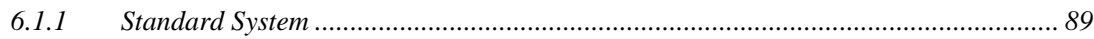

System for Volatile Organic Compounds............................................................. 90

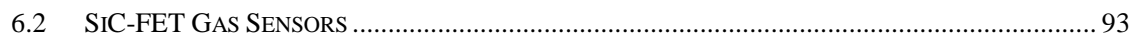

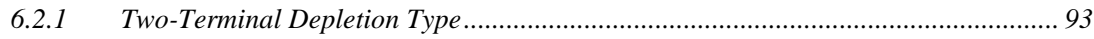

6.2.2 Two-Terminal Enhancement Type …..................................................................... 95

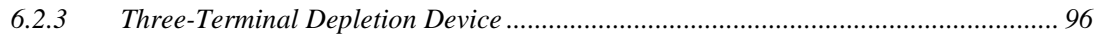

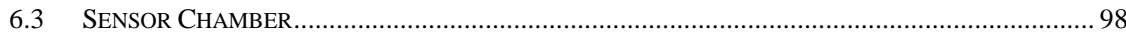

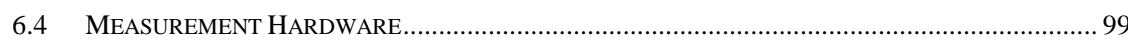

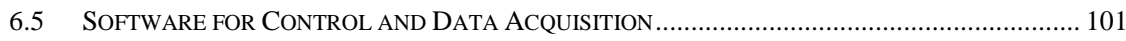

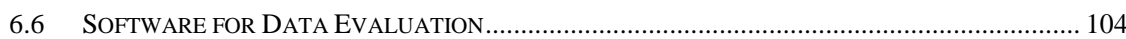

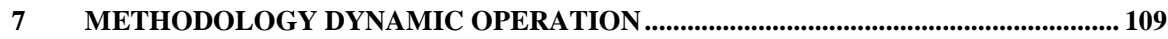

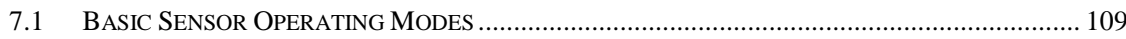

7.1.1 Constant Drain Current ..................................................................................... 110 


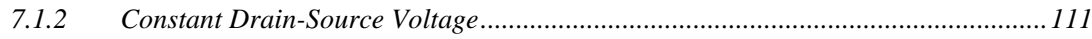

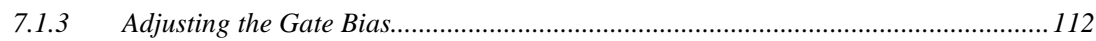

7.1.4 Conclusion Sensor Operating Mode …............................................................ 112

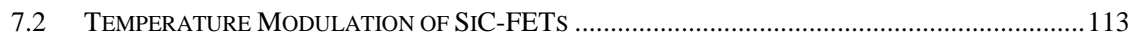

7.2.1 Temperature Cycle and Feature Extraction......................................................... 113

7.2.2 Pre-Processing and Feature Extraction ....................................................... 114

7.2.3 Discrimination and Quantification .................................................................... 116

7.2.4 Conclusion Temperature Modulation ....................................................................... 118

7.3 Gate Bias Modulation OF SiC-FETs .......................................................................... 119

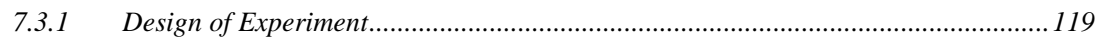

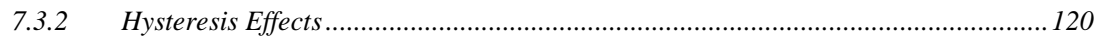

7.3.3 Explanations for Hysteresis ............................................................................... 122

7.3.4 Discrimination Using Hysteresis .................................................................... 124

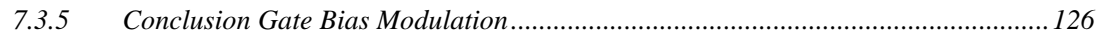

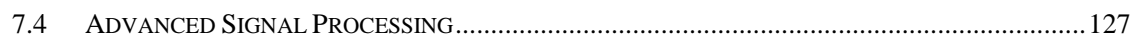

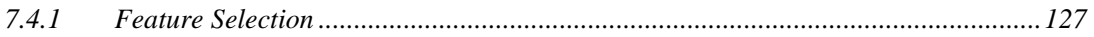

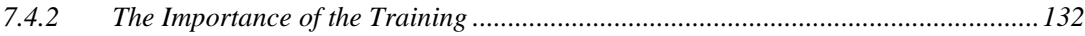

7.4.3 Hierarchical Discrimination.................................................................................. 133

7.4.4 Conclusion Advanced Signal Processing ........................................................... 135

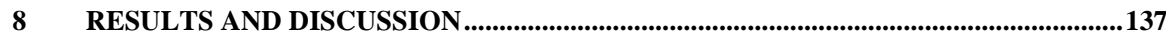

$8.1 \quad$ EXHAUST MONITORING - NO

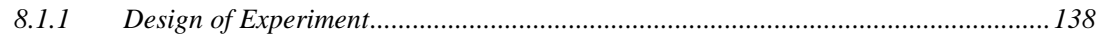

8.1.2 Temperature Cycle for $\mathrm{NO}_{x}$ Detection ................................................................... 140

8.1.3 Sensor Response and Signal Processing ........................................................... 141

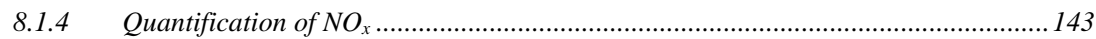

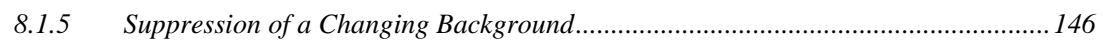

8.1.6 Conclusion $\mathrm{NO}_{x}$ Detection .................................................................................. 151

8.2 SiC-FETS FOR INDOOR AIR QUALITY APPLICATIONS ...................................................... 153

8.2.1 Detection of Volatile Organic Compounds ............................................................ 153

8.2.2 Discrimination of Volatile Organic Compounds.................................................... 157

8.2.3 Quantification of Volatile Organic Compounds .................................................... 162

8.2.4 Detection of VOC in High Background of Ethanol................................................. 168

8.2.5 Conclusion Detection of Volatile Organic Compounds ........................................... 172

8.3 COMBINATION OF TEMPERATURE AND GATE-BIAS CYCLING ............................................ 173

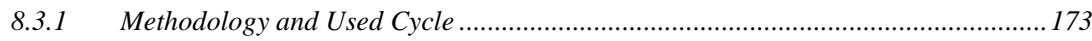

8.3.2 Quantification of Carbon Monoxide …................................................................ 175

8.3.3 Discrimination and Feature Selection .................................................................... 177

8.3.4 Feature Transformation by Principal Component Analysis .................................... 182

8.3.5 Stability and Influence of Normalization ................................................................. 186

8.3.6 Drift Compensation by Extended Training .............................................................. 188 
8.3.7 Conclusion Extended Virtual Multi-sensor Approach ......................................... 190

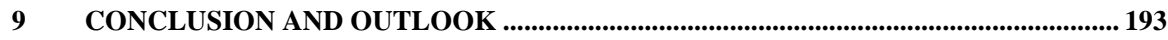

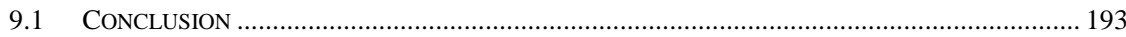

9.2 MY CONTRIBUTION TO THE SCIENTIFIC COMMUNITY ......................................................... 198

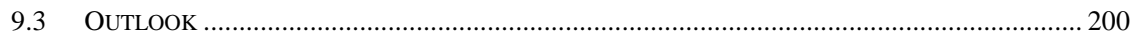

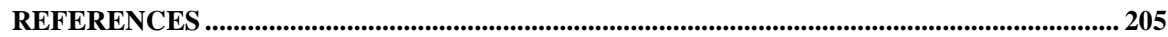

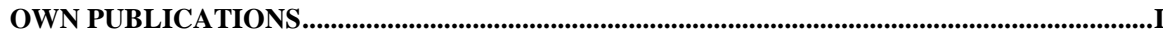

PEER-REVIEWED JOURNAL ARTICLES ...........................................................................................

MY CONTRIBUTION TO THESE JOURNAL ARTICLES ...................................................................IV

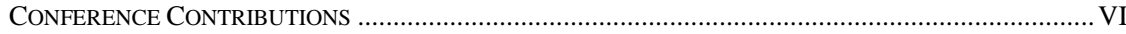

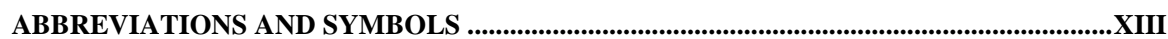

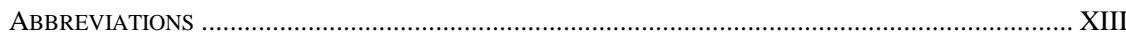

SENSOR DEVICE RELATED SYMBOLS .........................................................................................

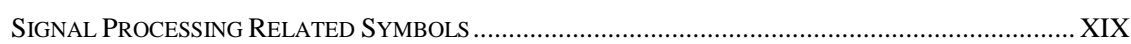

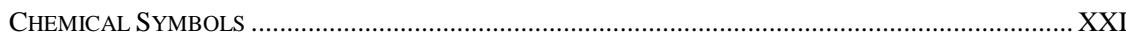

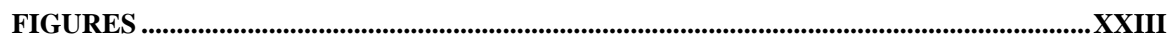

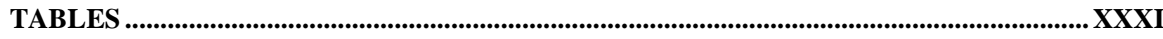

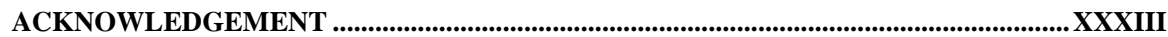

AFFIDAVIT / EIDESSTATTLICHE VERSICHERUNG ..............................................XXXIX 


\section{Introduction}

This first chapter gives a general introduction to gas sensors including different transducer principles and possible applications, cf. Section 1.1. After that, the scope of this thesis is briefly elucidated, cf. Section 1.2.

\subsection{Motivation}

Gas sensors are part of our daily life in various manners ranging from consumer goods, comfort and safety applications to quality assurance in modern production lines. Examples for consumer goods are air fresheners like the Air Wick Freshmatic, in which a gas sensor from AppliedSensor is applied to release fragrance automatically when needed [1]. A typical comfort application of gas sensors is automotive cabin air quality, CAQ [2], [3]. Sensors determine the quality of the air outside the cabin and close ventilation dampers if necessary. There is a tendency to smart systems which determine whenever the air quality inside the cabin is better or worse than outside and control the ventilation system accordingly. Typical target gases are carbon monoxide, hydrocarbons, and nitrogen dioxide [4], [5]. Other comfort applications are in the field of building technology and heating, ventilating, and air-conditioning, HVAC, systems [6]. For the latter one, not only the air quality is important but also the energy consumption of the HVAC system. However, more demanding are safety-critical applications like detection of fires [7], [8], [9], [10], explosives with, e.g., methane as a target gas [11], [12], or prevention of poisoning, e.g., by carbon monoxide. Furthermore, gas sensors are applied for detecting leakages from pipelines or chemical storages. Leak detection is also a major issue for quality assurance, e.g., in the pharmaceutical industry [13], [14] and for hydrogen detection for instance in automotive applications [15]. In the automotive sector, oxygen sensors, i.e., the well-known lambda probes are used to monitor the air-fuel- 
ratio which is needed in order to control a closed-loop feedback-control system for the fuel injection. Additionally, environmental applications like measuring air pollution have become of increasing interest in the last years [16]. In particular, mobile sensor systems and sensor networks are in great demand in order to, for instance, draw environmental / pollution maps, i.e., visualize spatial distribution of pollutants.

Various different kinds of gas sensors both physical and chemical with different transducer principles and materials have been developed over the years, cf. Table 1.

Table 1 Overview of different gas sensor principles. Adopted from [17].

\begin{tabular}{|c|c|c|}
\hline method & measurand & sensor type / transducer \\
\hline amperometric & current & electrochemical cells, EC \\
\hline \multirow{3}{*}{$\begin{array}{l}\text { field effect based / } \\
\text { potentiometric }\end{array}$} & work function & field effect transistors, FET \\
\hline & $\mathrm{I}-\mathrm{V}$ & capacitors \\
\hline & $\mathrm{C}-\mathrm{V}$ & Schottky diodes \\
\hline \multirow{6}{*}{ optical } & \multirow{6}{*}{$\begin{array}{l}\text { transmission / adsorption } \\
\text { intensity } \\
\text { refractive index }\end{array}$} & infrared, IR \\
\hline & & non-dispersive infrared, NDIR \\
\hline & & optical fibers \\
\hline & & fluorescence \\
\hline & & Fourier transformation infrared \\
\hline & & spectroscopy, FTIR \\
\hline \multirow{4}{*}{ gravimetric } & \multirow{4}{*}{ resonance frequency } & bulk acoustic waves, BAW \\
\hline & & surface acoustic waves, SAW \\
\hline & & quartz micro balance, QMB \\
\hline & & cantilever \\
\hline \multirow{2}{*}{ conductometric } & \multirow{2}{*}{ conductance / resistance } & metal oxide semiconductor, MOX \\
\hline & & conducting polymers, $\mathrm{CP}$ \\
\hline capacitive & capacitance & polymers \\
\hline calorimetric & temperature & pellistor \\
\hline
\end{tabular}

Typical parameters to describe a sensor are detection mechanism, sensitivity, crosssensitivity or selectivity, drift or long-term stability, power consumption, and costs. Depending on the target application, one or the other parameter dominates. For 
safety critical applications selectivity and long-term stability predominate whereas for consumer products the costs are the most demanding factor.

Solid state chemical gas sensors are typically inexpensive, quite sensitive, and easy to fabricate. Hence, they are attractive for many applications. However, the biggest challenges are selectivity and cross-sensitivity. To some extent the selectivity can be tuned by carefully choosing the sensing material, operating temperature, and maybe applying additional filters. Anyhow, a total selective chemical gas sensor does not exist. Much effort has been spent in the last decades on increasing the selectivity. A commonly used setup is a sensor array in which many broad-band sensors ${ }^{1}$ are combined. The idea is that the pattern of many different sensors provides a fingerprint of the test gas mixture similar as a human or animal nose. Thus, sensor arrays used in the field of olfaction are often called electronic noses, EN, and have been studied intensively in the 1990s and 2000s [18]. Electronic noses are used to detect odors and flavors in, e.g., the food industry. Various kinds of gas sensors are used in EN, e.g., FETs, MOX, CP, and QMB (cf. Table 1).

By using a sensor array, data evaluation becomes complex which calls for more sophisticated analysis. Typically, pattern recognition and machine learning algorithms are considered. With appropriate signal processing the selectivity of the sensor system can be increased considerably. Nevertheless, depending on the algorithm other problems, e.g., over-fitting might become an issue.

In analogy to EN, virtual arrays or virtual multi-sensors have been suggested based on resistive type metal oxide sensors. Instead of a sensor array, single sensors are used but with, e.g., a modulated operating temperature. Temperature modulation or temperature cycled operation, $\mathrm{TCO}$, is a powerful method to increase the selectivity of MOX sensors [19]. Besides TCO also electrical impedance spectroscopy, EIS, and pulsed operation have been suggested to gain more information with respect to selectivity. The virtual-multi sensors approach, with temperature modulation as the main method, has so far not been studied systematically in the area of field effect based gas sensing. In this project, the methodology of dynamic operation of gas sensitive field effect transistors is developed as well as an extension of signals processing strategies.

\footnotetext{
${ }^{1}$ Broad-band sensors are sensitive to various different gases and are thus, less specific.
} 


\subsection{Scope of This Thesis}

This thesis deals with the methodology of dynamic operation applied to gas sensitive silicon carbide field effect transistors, SiC-FETs. Dynamic operation of single sensors is known as the virtual multi-sensor approach. For resistive type metal oxide, MOX, gas sensors temperature cycled operation, TCO, is a well-known method to improve the selectivity and to some extent also the sensitivity. Gas sensitive field effect transistors, FETs, are another well-known, commercially available, type of chemical gas sensor. Especially, with silicon carbide, SiC, as the substrate material, these sensors are suitable candidates to operate in harsh environments. However, so far, dynamic operation of SiC-FETs has not been studied systematically.

The focus of the research, which has been performed, is on the development of a methodology to operate SiC-FETs dynamically as well as on appropriate signal processing strategies, mainly based on multivariate statistics. Thus, in this thesis thorough information regarding the $\mathrm{SiC}$ field effect gas sensor, state-of-the-art dynamic operation, and multivariate statistics is provided. In contrast to MOX sensors, FETs are much more complex and offer the possibility to vary other parameters than temperature, like the gate bias. In this thesis, the virtual multi-sensor approach is not only transferred to SiC-FET gas sensors but also extended by gate bias cycling. After developing the methodology and validating the concept, the virtual multi-sensor approach is applied to two different applications; $\mathrm{NO}_{\mathrm{x}}$ quantification in a varying background, and discrimination and quantification of ultra-low concentrations of volatile organic compounds, VOCs, for indoor air quality applications. Additionally, temperature and gate bias cycling are combined to boost the selectivity further as demonstrated by a more methodological study. 


\section{Air Pollutants}

Several studies have revealed that air pollution both outdoors and indoors have a serious impact on human health [20], [21]. Typically, the concentrations of pollutants indoors are much higher. The level of outdoor pollution depends strongly on the location; generally cities have larger problems than rural areas. On a global scale, half of the population lives in urban areas, in developed countries more than $75 \%$, and in developing countries approximately $60 \%$ [22]. Classical outdoor air pollutants are particulate matter, ozone, nitrogen dioxide, and sulfur dioxide [22]. Additionally, there are also organic pollutants, e.g., carbon monoxide or aromatic hydrocarbons, and inorganic pollutants, e.g., arsenic compounds, asbestos, or metals [23]. Typical sources are mainly exhaust emissions from traffic and heavy industry. Health effects are breathing problems, asthma and bronchitis, reduced lung function, irritations of the respiratory system, and irritation of the eyes [24]. However, also more severe problems like lung cancer or cardiovascular morbidity leading to an increased risk of mortality and reduced life expectancy have been reported [22], [25]. It has been estimated that there were 3.7 million premature deaths worldwide in 2012 due to air pollution [24].

Besides the outdoor air pollution, the quality of indoor air is more serious since people spend most of their time indoors where fresh air exchange is extremely limited. Main pollutants are carbon dioxide, $\mathrm{CO}_{2}$, and volatile organic compounds, VOC which are harmful even at very low concentrations. Typical health effects like sensory irritation may be associated with the exposure to VOCs, but also chronic toxic (non-cancer) and carcinogenic health effects are observed. Especially, kindergarten and school children are affected most by indoor air pollution and are also more sensitive [26], [27]. Indoor sources are building materials, furniture, cleaning products, cooking, heating, and tobacco smoke.

The following sections discuss the commonly observed air pollutants, their sources and health impacts more deeply. 


\subsection{Emission from Exhaust}

In the last years, the requirements for the reduction of emissions from traffic and power plants, e.g., carbon dioxide, hydrocarbons, particulate matter, and nitrogen oxides, have become more and more strict. Especially, the amount of nitrogen monoxides, $\mathrm{NO}$, and nitrogen dioxide, $\mathrm{NO}_{2}$, short $\mathrm{NO}_{\mathrm{x}}$, in the exhaust of diesel driven cars has been limited considerably in the latest emission standards. For instance, the Euro 6 standard which became active from September 1, 2014 on allows a maximal total $\mathrm{NO}_{\mathrm{x}}$ emission of $80 \mathrm{mg} / \mathrm{km}$ for diesel driven cars, whereas in the Euro 5 standard $180 \mathrm{mg} / \mathrm{km}$ are allowed [28].

In general, diesel driven cars emit lower amounts of carbon monoxide and unburned hydrocarbons compared to gasoline engines but emit higher amounts of particulates and nitrogen oxides [29]. Diesel particle filters, DPF, are applied to effectively reduce the amount of small particles in the exhaust. However, since the introduction of DPF to London buses, an increase in the $\mathrm{NO}_{2} / \mathrm{NO}$ ratio has been observed [30]. In a study by the King's College of London and the University of Leeds, it was reported that recent concentrations of $\mathrm{NO}_{\mathrm{x}}$ and $\mathrm{NO}_{2}$ in the $\mathrm{UK}$ have not decreased as anticipated. By looking at the emission of $\mathrm{NO}_{\mathrm{x}}$ as a function of the vehicle specific power, it was found that, Euro 3-5 diesel driven cars under higher engine load can emit up to twice the amount of $\mathrm{NO}_{\mathrm{x}}$ compared with older generation vehicles [31]. In a study of The European Environmental Agency, EEA, it is reported that in some European cities the amount of $\mathrm{NO}_{2}$ increased over the last years due to increasing number of modern diesel vehicles [32]. Nitrogen dioxide contributes directly to the formation of ground level ozone, better known as smog, and acid rain (formation of nitric acid).

In gasoline engine technology, the trend is pointing towards lean burn or lean combustion engines [33]. These engines operate with a significantly higher oxygenfuel ratio which enhances the efficiency of burning the fuel. The higher temperatures of a diesel engine and the lean conditions in gasoline engines lead to an increase in $\mathrm{NO}_{\mathrm{x}}$ formation. Under normal working conditions, it was proven that a three-way catalyst, TWC, works efficiently for the removal of $\mathrm{NO}_{\mathrm{x}}$ but under the new conditions TWC is completely ineffective [33]. 
Another strategy to reduce $\mathrm{NO}_{\mathrm{x}}$ is by adding ammonia, $\mathrm{NH}_{3}$, in form of urea to the exhaust; the hazardous $\mathrm{NO}_{x}$ can be reduced effectively to nitrogen and water in so called selective catalytic reduction, SCR, systems [29], [34]. However, in order to control SCR systems sensors are needed, which measure either the $\mathrm{NO}_{x}$ or $\mathrm{NH}_{3}$ concentration in the exhaust. 


\subsection{Indoor Air Quality}

The demand for intelligent ventilation systems for indoor air quality, IAQ, applications has increased considerably in the last years. People spend most of their time indoors, approximately $80 \%$ to $85 \%$ where fresh air exchange is usually extremely limited. Exposure to indoor air pollutants for a prolonged time can lead to serious health problems. Typical symptoms are acute discomfort, headaches, sensory irritations, dizziness, and difficulties in concentrating [35], [36]. These kinds of health issues are summarized under the term Sick Building Syndrome, SBS [37]. Commonly associated with IAQ and used as an indicator for most ventilation systems nowadays, is the level of carbon dioxide, $\mathrm{CO}_{2}$, in the air [38]. Elevated concentrations of around 1,000 ppm of $\mathrm{CO}_{2}$ cause, e.g., fatigue. Carbon dioxide can be measured using infrared, IR, spectroscopy [39]. Mainly non-dispersive infrared, NDIR, transmission sensors [40] and sensors based on photoacoustic adsorption are on the market [41]. However, more dangerous for the human health are volatile organic compounds, VOCs, especially since they not only lead to acute but also to chronic health effects [42]. Even at very low concentrations, typically in the partsper-billion, ppb, range they pose serious health risks [35], [43].

Currently, heating, ventilating, and air-conditioning, HVAC, systems are mainly used to control the temperature and humidity level. Additionally, ventilation can reduce the amount of air pollutants and, with that, the exposure of those to humans. However, these systems consume a considerable amount of energy. It was reported that HVAC systems in developed countries account for half of the energy used in buildings and one fifth of the total national energy [44]. The efficiency is greatly enhanced by using on-demand ventilation systems. However, in order to operate such systems, sensors measuring the air quality are required. The European Union has been supporting several initiatives in the last years dealing with sensor systems for IAQ applications. For instance the SENSIndoor project from the European Seventh Framework Program FP7/2007-2013, grant no. 604311, focuses on the development of novel sensor systems for health and energy optimized IAQ control ${ }^{2}$ [45].

\footnotetext{
${ }^{2}$ Results presented in Section 8.2 of this thesis are obtained at least partly within the SENSIndoor project.
} 
Different studies have addressed indoor air pollutants and suggested guideline and threshold values. The first indoor air quality guidelines, IAQGs, were suggested by the French Agency for Food, Environmental, and Occupational Health \& Safety, ANSES formally AFSSET, in 2006 [46].

Priority lists of indoor air pollutants with an undeniable health impact were suggested by ANSES and by the European INDEX project [55]. Table 2 summarizes typical indoor air pollutants and current guide levels are given.

Table 2 Indoor air pollutants of interest with highest relevance and current exposure limit. Reproduced from [47] with kind permission from IEEE.

\begin{tabular}{|c|c|c|c|c|c|c|}
\hline pollutant & exposure lim & & country & year & remarks & ref. \\
\hline benzene & $5 \mu \mathrm{g} \mathrm{m}^{-3}$ & $1.5 \mathrm{ppb}$ & France & 2013 & $\begin{array}{l}\text { Long term exposure } \\
\text { no safe level of exposure } \\
\text { (WHO) }\end{array}$ & $\begin{array}{l}{[48]} \\
{[42]}\end{array}$ \\
\hline formaldehyde & $\begin{array}{l}100 \mu \mathrm{g} \mathrm{m}^{-3} \\
50 \mu \mathrm{g} \mathrm{m}^{-3}\end{array}$ & $\begin{array}{l}80 \mathrm{ppb} \\
40 \mathrm{ppb}\end{array}$ & $\begin{array}{l}\text { Sweden } \\
\text { France }\end{array}$ & $\begin{array}{l}1987 \\
2007\end{array}$ & $\begin{array}{l}\text { adopted from WHO, } 30 \text { - } \\
\text { min average Short-term } \\
\text { (for exposure of } 2 \text { hours) }\end{array}$ & $\begin{array}{l}{[42]} \\
{[49]} \\
{[46]}\end{array}$ \\
\hline naphthalene & $30 \mu \mathrm{g} \mathrm{m}^{-3}$ & $5.6 \mathrm{ppb}$ & Germany & 2013 & Guide value II (RWII) & [50] \\
\hline carbon & $60 \mathrm{mg} \mathrm{m}^{-3}$ & $52 \mathrm{ppm}$ & Germany & 1997 & $30 \mathrm{~min}$ average, guide & [51] \\
\hline monoxide & $30 \mathrm{mg} \mathrm{m}^{-3}$ & $26 \mathrm{ppm}$ & France & 2008 & $\begin{array}{l}\text { value II (RWII) } \\
\text { 1-hour average }\end{array}$ & $\begin{array}{l}{[42]} \\
{[46]}\end{array}$ \\
\hline $\begin{array}{l}\text { nitrogen } \\
\text { dioxide }\end{array}$ & $200 \mu \mathrm{g} \mathrm{m}^{-3}$ & $105 \mathrm{ppb}$ & France & 2013 & $\begin{array}{l}\text { Short-term (for exposure } \\
\text { of } 2 \text { hours) }\end{array}$ & [52] \\
\hline radon & $167 \mathrm{~Bq} \mathrm{~m}^{-3}$ & & WHO & 2010 & $\begin{array}{l}\text { Annual average; excess } \\
\text { lifetime risk } \\
1 \text { per } 1000 \text { for non- } \\
\text { smokers }\end{array}$ & $\begin{array}{l}{[53]} \\
{[42]}\end{array}$ \\
\hline styrene & $70 \mu \mathrm{g} \mathrm{m}^{-3}$ & $16 \mathrm{ppb}$ & WHO & 2000 & 30-min average & [23] \\
\hline $\begin{array}{l}\text { tetrachloro- } \\
\text { ethylene }\end{array}$ & $0.25 \mathrm{mg} \mathrm{m}^{-3}$ & $36 \mathrm{ppb}$ & WHO & 2010 & Annual average & [42] \\
\hline toluene & $3 \mathrm{mg} \mathrm{m}^{-3}$ & $783 \mathrm{ppb}$ & Germany & 1996 & Guide value II (RWII) & [54] \\
\hline $\begin{array}{l}\text { trichloro- } \\
\text { ethylene }\end{array}$ & $230 \mu \mathrm{g} \mathrm{m}^{-3}$ & $42 \mathrm{ppb}$ & WHO & 2010 & $\begin{array}{l}\text { Excess lifetime cancer } \\
\text { risk 1:10,000 }\end{array}$ & [23] \\
\hline PM10 & $50 \mu \mathrm{g} \mathrm{m}^{-3}$ & & WHO & 2005 & 24-hour mean & [52] \\
\hline
\end{tabular}


From the ANSES studies, class A, compounds with the utmost priority contains among others the three VOCs formaldehyde, naphthalene, and benzene, cf. Fig. 2.1.

Sources, exposure levels, and health effects of these three VOCs are briefly summarized in the following sections. The elucidations are based on [47]. Further information can also be found in [56] and in the reference given in the sections.

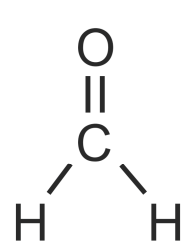

formaldehyde<smiles>c1ccccc1</smiles>

benzene

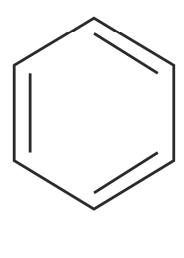

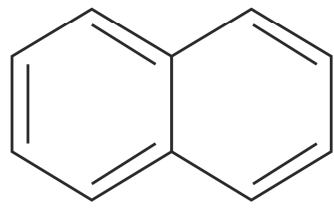

naphthalene

Fig. 2.1 Structural formula of formaldehyde, benzene, and naphthalene.

\subsubsection{Formaldehyde}

Formaldehyde $\left(\mathrm{H}_{2}-\mathrm{C}=\mathrm{O}\right.$, cf. Fig. 2.1, $\mathrm{CAS}^{3}$ number 50-00-0) is one of the most well-known VOCs and very common in indoor air. The official IUPAC ${ }^{4}$ name is methanal; other names are methyl aldehyde, methylene glycol, and formalin. It has a molar mass of $30.03 \mathrm{~g} / \mathrm{mol}$ and a high vapor pressure of $0.5 \mathrm{MPa}$ at $25^{\circ} \mathrm{C}$. Formaldehyde is formed both naturally and synthetically, and is thus ubiquitous in the environment. Natural sources are combustion or decomposition of biomass and through volcanic eruptions [42]. However, the main outdoor sources arise from extensive use in industrial processes, e.g., production of resins, binders for wood products, paper or pulp, and the exhausts of combustion processes, mainly vehicle and air traffic [57]. Outdoor concentrations are typically below $100 \mu \mathrm{g} / \mathrm{m}^{3}$ in rural environments and around $200 \mu \mathrm{g} / \mathrm{m}^{3}$ in highly urbanized or industrial areas [42]. Formaldehyde is also contained and released from many building materials and consumer products which are one main source in particular for indoor environments. Other sources are smoking and cooking but also do-it-yourself products and household cleaning products. Especially, outgassing from wood-based materials (among others pressed wood products), glues, laminate floors, and coatings including wallpaper are

\footnotetext{
${ }^{3} \mathrm{CAS}$ number is a unique identification number assigned by Chemical Abstracts Service for every chemical.

${ }^{4}$ IUPAC - International Union of Pure and Applied Chemistry
} 
of major concern [57] as well as formaldehyde releasing from textiles are also a main concern [58], [59].

Indoor levels of formaldehyde vary significantly depending on location, e.g., residential areas or workplace: The mean concentrations in living areas are typically between 18 and $45 \mu \mathrm{g} / \mathrm{m}^{3}$, with maximum concentrations up to several hundred $\mu \mathrm{g} / \mathrm{m}^{3}[42]$.

Health effects are mainly sensory irritation of the eyes and mucous membranes [60]. Formaldehyde is also classified as carcinogenic for humans by the WHO. Short-term, i.e., 30-minute, limit of $100 \mu \mathrm{g} / \mathrm{m}^{3}$, approximately $80 \mathrm{ppb}$, for preventing sensory irritation in general population is recommended by the WHO [23], [42]. In Germany, guide level of $100 \mathrm{ppb}$ is valid [49], and in France, a 2-hour limit value of $50 \mu \mathrm{g} / \mathrm{m}^{3}$, approximately $40 \mathrm{ppb}$, has been introduced [46].

\subsubsection{Benzene}

Benzene $\left(\mathrm{C}_{6} \mathrm{H}_{6}\right)$ is the simplest aromatic hydrocarbon, cf. Fig. 2.1. The official IUPAC name is Cyclohexa-1,3,5-triene (CAS number 71-43-2). It has a molar mass of $78.1 \mathrm{~g} / \mathrm{mol}$ and a high vapor pressure of $12.7 \mathrm{kPa}$ at $25^{\circ} \mathrm{C}$ causing it to evaporate rapidly at room temperature. Benzene is typically used as an intermediate or precursor in the synthesis of other chemicals, in particular ethylbenzene, cumene, cyclohexane, and styrene. It is also contained up to $1 \%$ in gasoline. Thus, typical outdoor sources are emissions from petrochemical industry, traffic, and gas stations. Typical outdoor concentrations vary from sub-ppb, in rural areas over low-ppb, in urban areas to tens of ppb in source impacted areas [23], [61]. Benzene is also present indoors and these concentrations are generally higher than outdoors [42]. Indoor sources are building materials, furniture, including furniture wax, paints, and glues. Additionally, benzene is contained in tobacco smoke and released by heating and cooking systems [42]. It was reported that typical indoor concentrations in Europe are in the range from $2 \mu \mathrm{g} / \mathrm{m}^{3}$ (ca. $0.6 \mathrm{ppb}$ ) in Finland up to $14 \mu \mathrm{g} / \mathrm{m}^{3}$ (ca. $4 \mathrm{ppb}$ ) in Turkey [42]. However, much higher levels are sometimes also found, e.g., 18 to $35 \mu \mathrm{g} / \mathrm{m}^{3}$ in buildings in Singapore. Indoor concentration of benzene depends also on the outdoor concentration and the air exchange rate. The level of benzene can be greatly higher when a garage is attached to the living area. When 
entering the garage itself, the short-term exposure can be tens to hundreds of ppb [61].

Even at very low concentrations, benzene poses serious health risks causing both non-carcinogenic and carcinogenic effects. The most adverse health effects are haematotoxicity, genotoxicity, and carcinogenicity [23]. Carcinogenic effects like acute myeloid leukemia and genotoxicity were reported [42]. Benzene is classified as carcinogenic for humans and no safe level of exposure can be recommended. The concentrations of airborne benzene associated with a risk of reduced lifetime of $10^{-4}$, $10^{-5}$, and $10^{-6}$ are $17,1.7$, and $0.17 \mu \mathrm{g} / \mathrm{m}^{3}$, respectively [42], [23]. Therefore, the exposure to benzene should be reduced as much as possible.

Recently, regulations of the level of benzene in public buildings have been established in France [48]. These regulations demand a threshold of $1.5 \mathrm{ppb}$ in 2013 which is decreased to $0.6 \mathrm{ppb}$ by 2016 .

\subsubsection{Naphthalene}

Naphthalene is the simplest polycyclic aromatic hydrocarbon $\left(\mathrm{C}_{10} \mathrm{H}_{8}\right)$, cf. Fig. 2.1, with a molar mass of $128.17 \mathrm{~g} / \mathrm{mol}$ and a vapor pressure of $10 \mathrm{~Pa}$ at $25^{\circ} \mathrm{C}$. The official IUPAC name is Bicyclo[4.4.0]deca-1,3,5,7,9-pentene (CAS number 91-20-3). It is mainly used as starting material in the manufacture of phthalic anhydride in the production of plasticizers, as ingredient for plasterboards, and as tanning agent in the leather industry [42]. Further, naphthalene is used in paints, resins, and the production of insecticides [42], [62]. Besides outgassing of consumer and building products, main sources of exposure today are incomplete combustion processes, e.g., vehicle and air traffic, residential heating, and tobacco smoke. Formerly, it was also used as insecticide in mothballs.

Outdoor concentrations in rural areas are ranging from 1 to $4 \mu \mathrm{g} / \mathrm{m}^{3}$, i.e., less than $1 \mathrm{ppb}$, in Europe [42] up to $170 \mu \mathrm{g} / \mathrm{m}^{3}$ in large cities [62]. However, indoor levels of naphthalene can be significantly higher which is caused by outgassing of consumer products such as multipurpose solvents, lubricants, herbicides, charcoal lighters, hair sprays, tobacco smoke, rubber materials, and mothballs [42], [62]. In several studies, it was reported that the mean value in Europe is usually low, $1-2 \mu \mathrm{g} / \mathrm{m}^{3}$, however individual samples with concentrations ranging from $0.7-14 \mu \mathrm{g} / \mathrm{m}^{3}$ were reported, 
too [42]. In studies of the European INDEX project [42], [55] it was mentioned that in some cities, for example in Athens, mean values up to $90 \mu \mathrm{g} / \mathrm{m}^{3}$ were measured. Exposure to volatile organic compounds at work, i.e., occupational exposure, is often several orders of magnitude higher than in living rooms [62].

Both acute and chronic health effects on humans were found. Inhalation of naphthalene causes, e.g., headaches, nausea, vomiting, and dizziness [63]. Other health effects are mostly respiratory tract lesions. Naphthalene is classified as a possible carcinogenic [42], [62]. In Germany, a guidance limit level (guide level I) of $0.01 \mathrm{mg} / \mathrm{m}^{3}$ corresponding to $3.5 \mathrm{ppb}$ is suggested, and a guide level II of $0.03 \mathrm{mg} / \mathrm{m}^{3}$, corresponding to $5.6 \mathrm{ppb}$ [50]. Guide level II represents the concentration of a substance which, if reached or exceeded, requires immediate action as this concentration could pose a health hazard [64]. 



\section{Field Effect Based Gas Sensing}

This chapter deals with the basics of field effect based gas sensors. First, the transducer principles of metal insulator semiconductor, MIS, capacitors and metal insulator semiconductor field effect transistors, MISFET, from a physical and electrical point of view are given in Section 3.1. In Section 3.2, the development of field effect based gas sensors is presented, followed by a discussion on surface chemistry in Section 3.3, and the sensing mechanisms in Section 3.4. At the end of the chapter (Section 3.5), some commonly used terms related to gas sensing are introduced and defined.

\subsection{Transducer Principle}

At first, the metal insulator semiconductor capacitor which is the heart of every field effect device is discussed. Then, the metal insulator field effect transistor as the ultimate transducer platform is described.

\subsubsection{Metal Insulator Semiconductor Capacitors}

The metal insulator semiconductor, MIS, capacitor, in some text books also called MIS diode [65], is a simple two-terminal MIS structure and represents the heart of most field effect devices including the commonly used field effect transistor, FET. The physics of MIS capacitors is well treated in semiconductor physics books by, e.g., Sze [65] or Neamen [66] as well as in certain chapters in sensor books [67], [68]. In this section, only the basic physical principles which are important for the understanding of field effect-based gas sensors are given. All 
considerations are based on a p-type semiconductor substrate ${ }^{5}$ and are mainly based on the books by Sze and Neamen.

Fig. 3.1a shows the schematic of an MIS structure on a p-type substrate with acceptor concentration $N_{A}$, insulator (oxide) thickness $d_{o x}$ and permittivity $\varepsilon_{o x}$. The capacitance per unit area $C_{\mathrm{OX}}^{\prime}$ is defined as:

$$
C_{\mathrm{ox}}^{\prime}=\frac{\varepsilon_{o x}}{d_{o x}}
$$

The energy band diagram of an ideal MIS structure is shown in Fig. 3.1b.

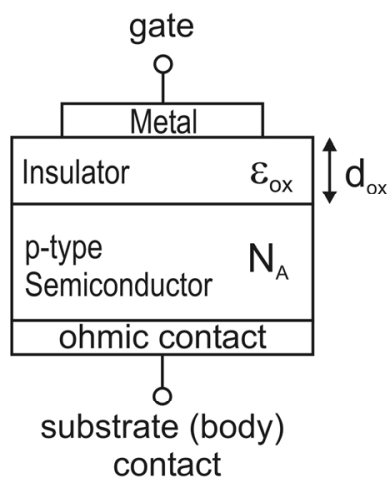

(a)

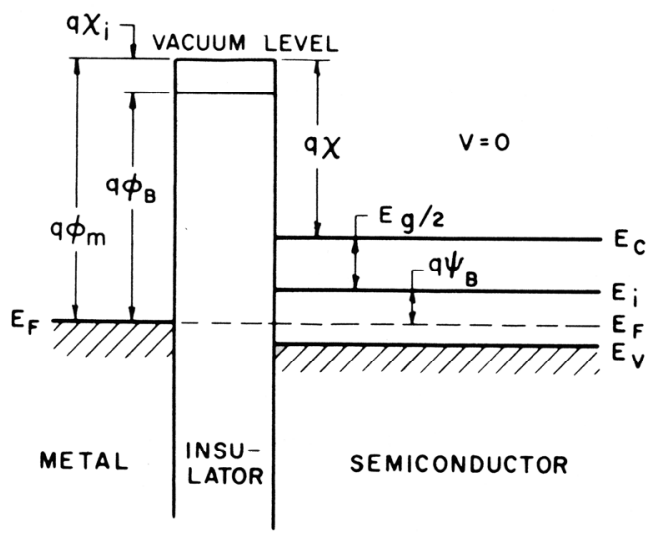

(b)

Fig. $3.1 \quad$ (a) Ideal p-type MIS structure. (b) Ideal energy band diagram of an ideal MIS structure under flat band condition. Reproduced from [65] with kind permission from Wiley.

An ideal MIS structure is defined as, after [65]: (1) at zero applied bias the difference of the metal and semiconductor work function $\phi_{m s}$ is zero, i.e., the energy bands are flat, known as flat-band condition. (2) There is no net charge in the semiconductor and (3) there is no carrier transport through the insulator under any DC bias condition. This assumptions lead to

$$
\phi_{m s}=\phi_{m}-\left(\chi+\frac{E_{g}}{2 \cdot q}+\psi_{B}\right) \stackrel{\text { ideal MIS }}{\longrightarrow} 0
$$

\footnotetext{
${ }^{5}$ Derivation for n-type can be found in literature.
} 
where $\phi_{m}$ is the metal work function which is the energy needed to move one electron from the conduction band to the vacuum level, $\chi$ the semiconductor electron affinity, $E_{g}$ the band gap of the semiconductor, $\psi_{B}$ the potential difference between the Fermi level and the intrinsic Fermi level, also known as the built in potential, and $q$ the elementary charge, cf. Fig. 3.1b.

The simplified model of an ideal MIS structure serves as a foundation to explain the behavior of MIS structures under certain bias conditions and the resulting effects.

If a negative bias is applied to the metal plate, i.e., $V<0$, a negative charge is deposited on the surface, which induces an electrical field pointing from the substrate to the top electrode. This field causes a band bending and the edge of the valence band at the insulator-semiconductor interface bends upwards in the direction of the Fermi level, cf. Fig. 3.2a. The Fermi level in the semiconductor remains constant for an ideal MIS structure due to the fact that no current flows into the structure, implying enrichment of majority carriers, i.e., holes at the surface of the semiconductor. This situation is known as accumulation. The energy difference of the Fermi level in the metal and in the semiconductor corresponds to the applied bias.

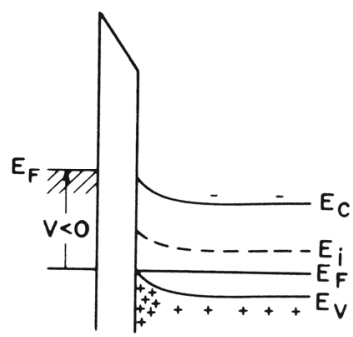

(a)

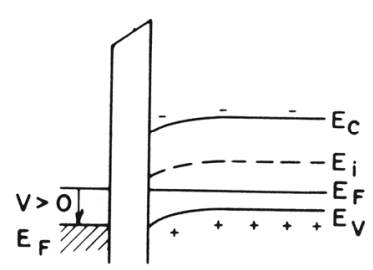

(b)

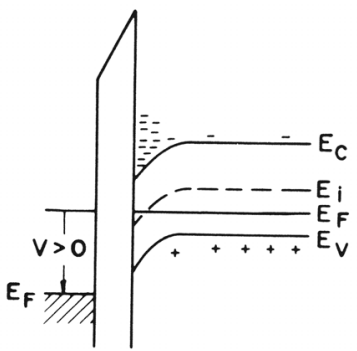

(c)

Fig. 3.2 Energy band diagram of an ideal MIS structure under certain bias. (a) When a negative bias, i.e., $\mathrm{V}<0$, (b) when a positive bias, i.e., $\mathrm{V}>0$, and (c) when a large positive bias, i.e., $\mathrm{V} \gg>0$ is applied. Reproduced from [65] with kind permission from Wiley.

When the polarity changes, i.e., a small positive bias, $V>0$, is applied, the bands bend downwards and the edges of the conduction band in the intrinsic Fermi level move closer to the Fermi level in the semiconductor, cf. Fig. 3.2b. Hence, the majority carriers are depleted from the semiconductor surface and a depletion region 
close to the surface is created. In other words, the density of holes at the surface is now lower than for the bulk. This case is known as depletion.

If the positive bias is further increased, the bands bend even more downwards so that the intrinsic Fermi level crosses over the Fermi level at the insulator-semiconductor interface, cf. Fig. 3.2c. At this point, inversion starts and the number of minority carriers, i.e., electrons, at the surface becomes larger than the number of majority carriers, i.e., holes. An inversion region is formed.

The band bending correlates directly to the surface potential $\psi_{S}$ which is defined as the difference of the intrinsic Fermi level at the surface and the bulk value, cf. Fig. 3.3.

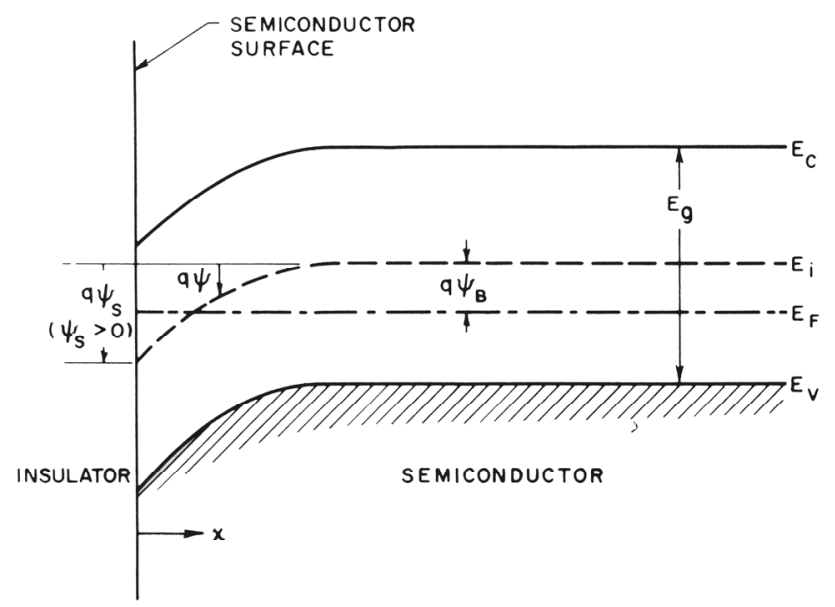

Fig. 3.3 Energy-band diagram at the surface of a p-type semiconductor. Reproduced from [65] with kind permission from Wiley.

Strong inversion takes place when the electron concentration at the surface is the same as the hole concentration in the bulk. In other words, the intrinsic Fermi level at the surface is $\psi_{B}$ below the Fermi level at surface, i.e., $\psi_{S}=2 \cdot \psi_{B}$. This condition is known as the threshold inversion point and the corresponding applied bias is the threshold voltage $V_{\text {th }}$ [66], also known as turn on voltage [65]. The threshold voltage can be calculated according to Eq. (3.3)

$$
V_{t h}=V_{o x}+2 \cdot \psi_{B}+\phi_{m s} \stackrel{\text { ideal MIS }}{\longrightarrow} V_{o x}+2 \cdot \psi_{B}
$$


where $V_{o x}$ is the voltage drop over the oxide at the threshold inversion point, $\psi_{B}$ the built in potential, and $\phi_{m s}$ the work function difference between the metal and the semiconductor which is considered to be zero for an ideal MIS structure.

The regions defined above can also be expressed with the help of the surface potential, after [65]:

$$
\begin{array}{ll}
\psi_{S}<0 & \text { accumulation of holes, i.e., band bending upwards } \\
\psi_{S}=0 & \text { flat-band condition, i.e., no band bending } \\
\psi_{B}>\psi_{S}>0 & \text { depletion of holes, i.e., band bending downwards } \\
\psi_{S}=\psi_{B} \quad \text { mid gap } \\
\psi_{S}>\psi_{B} \quad \text { inversion (electron enhancement) } \\
\psi_{S}=2 \cdot \psi_{B} \quad \text { threshold inversion point }
\end{array}
$$

The maximum width of the surface depletion region $W_{\max }^{\prime}$ can be defined as (for a detailed derivation see [65]):

$$
W_{\max }^{\prime}=\sqrt{\frac{2 \cdot \varepsilon_{o x} \cdot \psi_{S}(\text { inversion })}{q \cdot N_{A}}}=\sqrt{\frac{4 \cdot \varepsilon_{o x} \cdot \psi_{B}}{q \cdot N_{A}}}
$$

The voltage $V_{o x}$ is given as the charge on the metal surface $Q_{M}$ and the capacitance of the insulator $C_{o x}$. Due to charge neutrality, the charge on the metal surface is equal to the total charge in the semiconductor $Q_{S}$ which can be defined at the onset of strong inversion as $Q_{S}=q \cdot N_{A} \cdot W^{\prime}$. The threshold voltage can then be written as:

$$
V_{t h}=\frac{Q_{M}}{C_{o x}}+2 \cdot \psi_{B}=\frac{Q_{S}}{C_{o x}}+2 \cdot \psi_{B}=\frac{q \cdot N_{A} \cdot W^{\prime}}{C_{o x}}+2 \cdot \psi_{B}
$$

Equation (3.2) together with Eq. (3.5) and Eq. (3.6) derives the threshold voltage:

$$
V_{t h}=\frac{\sqrt{4 \varepsilon_{o x} \cdot q \cdot N_{A} \cdot \psi_{B}}}{C_{o x}}+2 \cdot \psi_{B}+\phi_{m s}
$$

So far, only ideal MIS structures have been considered where the work function difference between the metal and the semiconductor has been assumed to be zero. However, for practical MIS structures, i.e., in thermal equilibrium and without any 
applied bias, there is usually a work function difference, i.e., $\phi_{m s} \neq 0$, and also charges trapped in the insulator. Due to the fact that the Fermi levels of the individual parts are aligned, a band bending at the insulator-semiconductor surface is caused. The applied bias which is needed to compensate the band bending is called the flat band voltage $V_{F B}$

$$
V_{F B}=\left(\phi_{m}-\phi_{s}\right)-\frac{Q_{s}}{C_{o x}}=\phi_{m s}-\frac{Q_{s}}{C_{o x}}
$$

where $\phi_{m}$ and $\phi_{s}$ are the metal and semiconductor work functions and $Q_{s}$ is the sum of effective net insulator (oxide) charge per unit area and $C_{o x}$ the capacitance of the insulator.

In order to retrieve the capacitance-voltage relationship of an ideal MIS capacitor, i.e., when no charge is trapped in the insulator, one needs to consider the three different operating conditions discussed earlier again. In general, the capacitance $C$ is defined as

$$
C=\frac{d Q(V)}{d V}
$$

where $d Q$ is the differential change in charge on the metal divided by the differential change in voltage $d V$ across the device [66]. The capacitance is a function of the applied bias and is usually measured by superposing a small AC voltage.

In case of accumulation (for p-type semiconductor negative bias, $V<0$ ), holes are accumulated at the insulator-semiconductor interface. A small change in the applied bias, e.g., a superposition of a small AC voltage for measuring the capacitance, will cause a small change in electron concentration at the metal-insulator interface and likewise the same change of hole concentration at the insulator-semiconductor interface. The capacitance is therefore just the insulator capacitance, cf. Fig. 3.4.

$$
\mathrm{C}(\text { accumulation })=C_{o x}=\frac{\varepsilon_{o x}}{d_{o x}}
$$

If a small positive bias, $V>0$, is applied, a depletion region of majority carriers, i.e., holes, is created at the semiconductor surface. A small change in the applied voltages will change the width of the depletion region. The capacitance is then the capacitance 
of the insulator $C_{o x}$ in series to the capacitance of the depletion region $C_{D R}$, cf. Fig. 3.4.

$$
\frac{1}{\mathrm{C}(\text { depletion })}=\frac{1}{C_{o x}}+\frac{1}{C_{D R}}
$$

Since $C_{o x}=\varepsilon_{o x} / d_{o x}$ and $C_{D R}=\varepsilon_{s} / W^{\prime}$ with the permittivity of the semiconductor $\varepsilon_{s}$ and the width of the depletion region $W^{\prime}$, the total capacitance in depletion can be written as:

$$
\mathrm{C}(\text { depletion })=\frac{C_{o x} \cdot C_{D R}}{C_{o x}+C_{D R}}=\frac{\varepsilon_{o x}}{d_{o x}+\left(\frac{\varepsilon_{o x}}{\varepsilon_{S}}\right) \cdot W^{\prime}}
$$

The threshold inversion point (large positive bias) was defined as the point where the depletion width reaches its maximum but no inversion layer is created yet.

Thus, at the threshold inversion point, the capacitance is minimal, cf. Fig. 3.4.

$$
C_{\text {min }}=\frac{\varepsilon_{o x}}{d_{o x}+\left(\frac{\varepsilon_{o x}}{\varepsilon_{s}}\right) \cdot W_{\text {max }}^{\prime}}
$$

If the bias is increased further, an inversion layer is created at the insulatorsemiconductor surface while the width of the depletion region remains constant. If there is a small, low frequency change in the bias, e.g., due to the AC read-out of the capacitance, the depletion region does not change while the change in the inversion layer is equal to the change in charge at the metal-insulator interface. Hence, the capacitance is again given by the capacitance of the insulator $C_{o x}$, cf. Fig. 3.4, for frequencies in the range of 5 to $100 \mathrm{~Hz}$ [66]. In general, increase in capacitance depends on the ability of the electron concentration to follow the applied AC signal [65]. For high frequencies, typically in the order of $1 \mathrm{MHz}$, the electron concentration in the insulator, i.e., the inversion layer charge, cannot respond that quickly, hence the capacitance is again $C_{\min }$ as given in Eq. (3.13). Normally, MIS capacitors are measured at high-frequencies. 


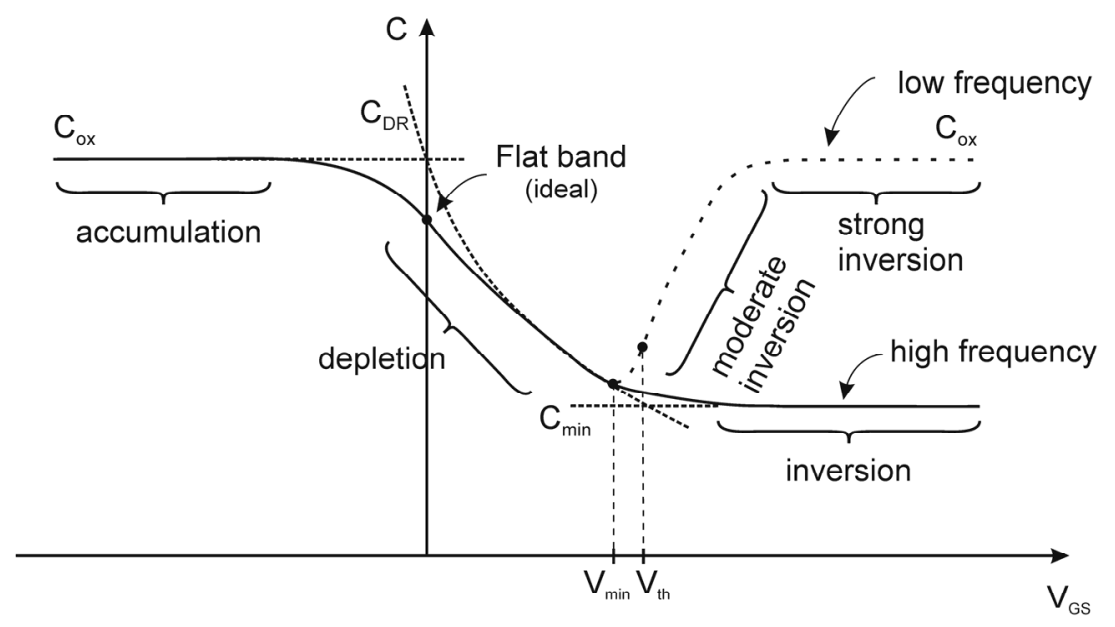

Fig. 3.4 Capacitance-voltage curve for an ideal MIS capacitor with p-type substrate. Adapted from [65] and [66].

In all previous discussions, an ideal oxide was assumed where for instance no interface trapped charges or fixed oxide charges were taken into account.

Generally, there are four different kinds of charge as shown in Fig. 3.5, after [65]: (i) interface trapped charges $Q_{i t}$, (ii) fixed oxide charges $Q_{f}$, (iii) oxide trapped charges $Q_{o t}$, and (iv) mobile ionic charges $Q_{m}$. Charges at the metal-insulator interface $Q_{i t}$ exist usually in the forbidden band-gap due to the fact that the periodic nature of the lattice terminates abruptly at the interface. A donor interface trap is usually located below the Fermi level and can become neutral by donating an electron. Acceptor interface traps exist in the upper half of the band gap and can become neutral by accepting an electron [65]. When a voltage is applied, interface traps move up and down with the valance and conduction band while the Fermi level remains constant. If the interface trap crosses the Fermi level, a change of charge occurs, i.e., charge flow from / to the oxide to / from the interface, and alters the ideal MIS curve. Oxide charges $Q_{S}$ including oxide fixed charge $Q_{f}$, oxide trapped charge $Q_{i t}$ and mobile ionic charge $Q_{m}$ are not a function of the applied gate bias. However, they influence the flat band voltage as mentioned earlier, cf. Eq. (3.8):

$$
V_{F B}=\phi_{m s}-\frac{Q_{f}+Q_{m}+Q_{o t}}{C_{o x}}=\phi_{m s}-\frac{Q_{s}}{C_{o x}}
$$




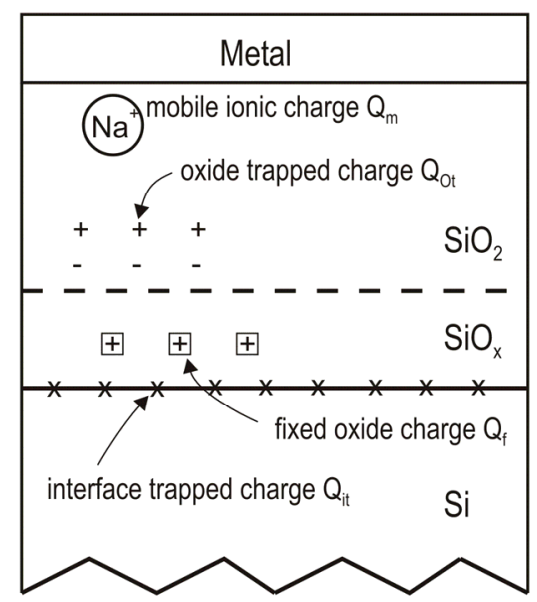

Fig. 3.5 Overview of different kind of charge. Adapted from [65].

Hence, the CV-curve remains the same as for an ideal MIS structure but with a parallel shift as depicted in Fig. 3.6.

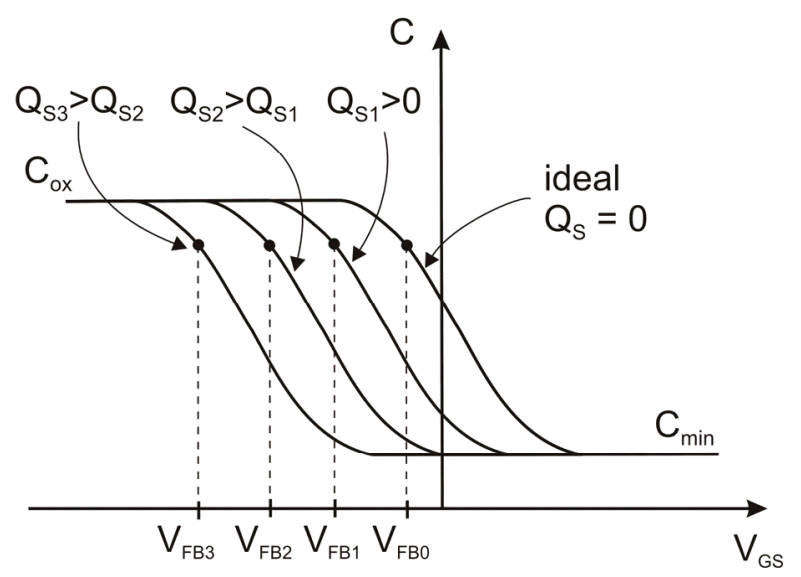

Fig. 3.6 High-frequency CV-curve of a MIS structure with effective trapped oxide charge. Adapted from [66]. 


\subsubsection{Metal Insulator Semiconductor Field Effect Transistors}

The earlier discussed MIS capacitor represents the heart of every metal insulator semiconductor field effect transistor, MISFET. In addition, several different types of FETs including junction FET, JFET, metal-semiconductor FET, MESFET, buried and short channel devices have been suggested. Here, only the basics of a classic MISFET are given which are important for the understanding of a gas sensitive FET. The physics of MISFETs is well treated in the semiconductor physics books by Sze [65] or Neamen [66] and, more from an engineering point of view in the books by Tietze and Schenk [69] or Horowitz and Hill [70]. Lundström et al. [67] and Andersson et al. [68] discuss the basics of MISFETs in relation to chemical sensors. The following explanations are mainly based on the books by Sze and Neamen.

\subsubsection{Transducer Platform}

A MISFET in the most general case is a four terminal device as shown in Fig. 3.7. The contacts are drain, source, gate, and substrate.

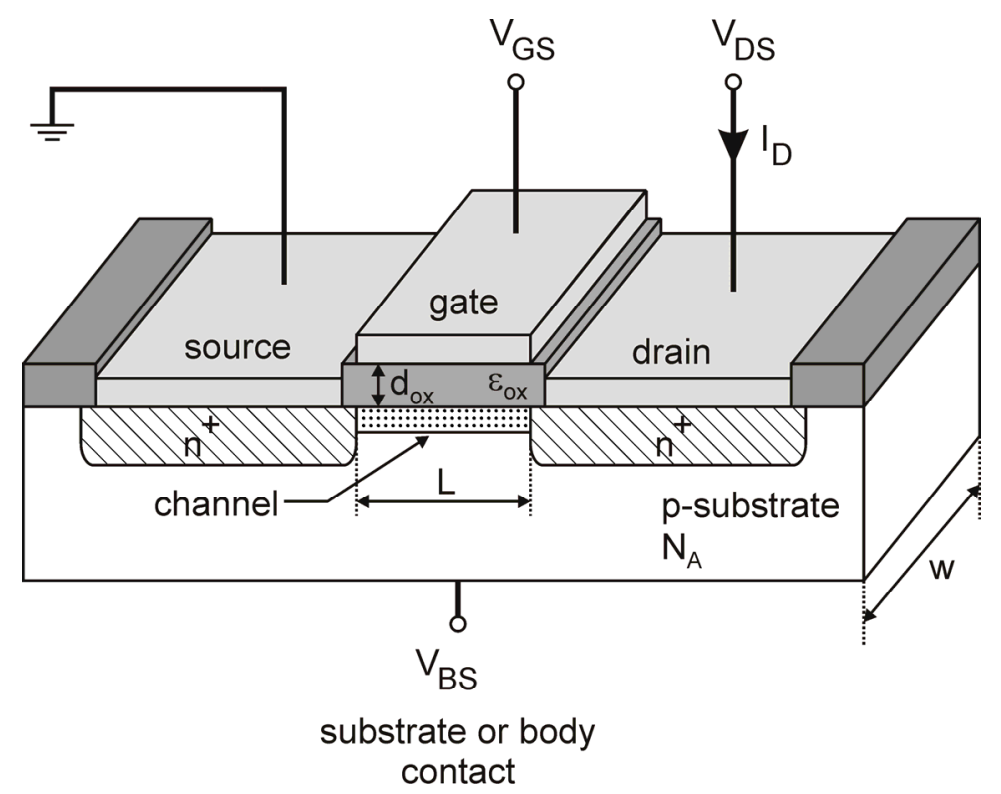

Fig. 3.7 Schematic diagram of a MISFET. Adapted from [65].

However, since the substrate, sometimes also called body or bulk, and source are usually connected and grounded; such devices are referred to as three terminal 
devices. The shown device has a p-type semiconductor substrate into which two high $\mathrm{n}$-doped $\left(\mathrm{n}^{+}\right)$regions, drain and source, are formed. Hence, this is an n-channel MISFET or n-MISFET. All further considerations are based on an n-channel enhancement type MISFET. A p-channel MISFET can easily be derived by exchanging $p$ for $n$ and reversing the polarity of the voltages. The terminal source is the source of charge carriers (electrons in case of an n-MISFET) that flow through the channel to the drain terminal. Yet, the convention for the direction of the drainsource current $I_{D}$ is from drain to source.

The metal contact on top of the insulator is called gate and plays a leading role for making an ordinary MISFET gas sensitive, see Section 3.2.1. The basic device parameters are the channel length $L$, which is the distance between the two $\mathrm{n}^{+}$regions ( $\mathrm{n}^{+}-\mathrm{p}$ junctions), the channel width $W$, the insulator thickness $d_{o x}$, and the substrate (semiconductor) doping $N_{A}$ [65]. In semiconductor industry especially the ratio between the width and the length $W / L$ is a key parameter.

In general, there are four different types of MISFETs which are shown in Fig. 3.8. Besides the substrate material, one distinguishes between normally-off or enhancement type and normally-on or depletion type devices. Normally-off means that with zero applied gate bias no channel between source and drain is created, i.e., the idea of the enhancement mode notation is that the semiconductor substrate is not inverted directly under the oxide [66]. For enhancement type FETs, the threshold voltage or turn on voltage is positive and for depletion type FETs, the threshold voltage is negative. Considering an enhancement type FET, when a sufficiently large gate bias, i.e., $V_{G S}>V_{t h}$, is applied, an n-type inversion layer between the two $\mathrm{n}^{+}$regions is created so that a conducting channel is formed on the surface of the semiconductor, allowing electrons to flow between source and drain.

The conductance of the channel can be modulated by the applied gate bias [65]. Also the substrate bias has an influence on the conductance and can be used as a voltage reference (as discussed later on in this section). On the other hand, for depletion type FETs an inversion layer, i.e., channel, already exists at zero applied gate bias.

Now, non-equilibrium conditions, i.e., when a voltage across the drain-source terminal is applied, will be considered and current-voltage relationships will be given. Assuming that an inversion layer between drain and source exist, 
i.e., $V_{G S}>V_{t h}$ (for enhancement type n-FET that requires a positive gate voltage) and a small drain-source voltage is applied, a current between drain and source will flow through the conducting channel.

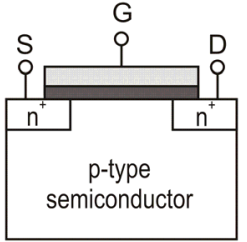

enhancement

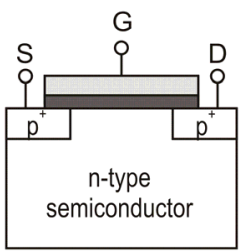

enhancement

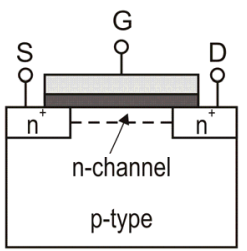

depletion

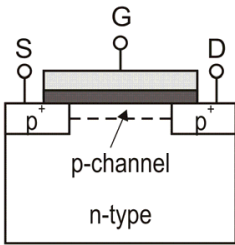

depletion
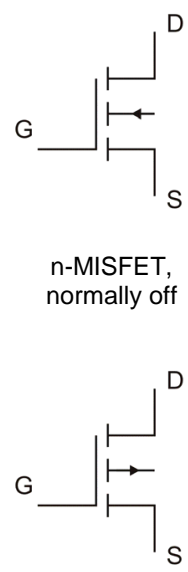

p-MISFET, normally off
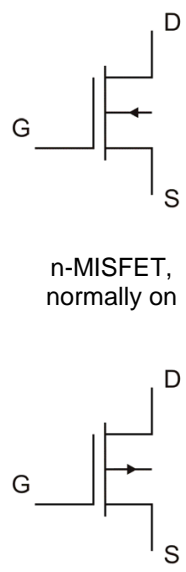

p-MISFET, normally on
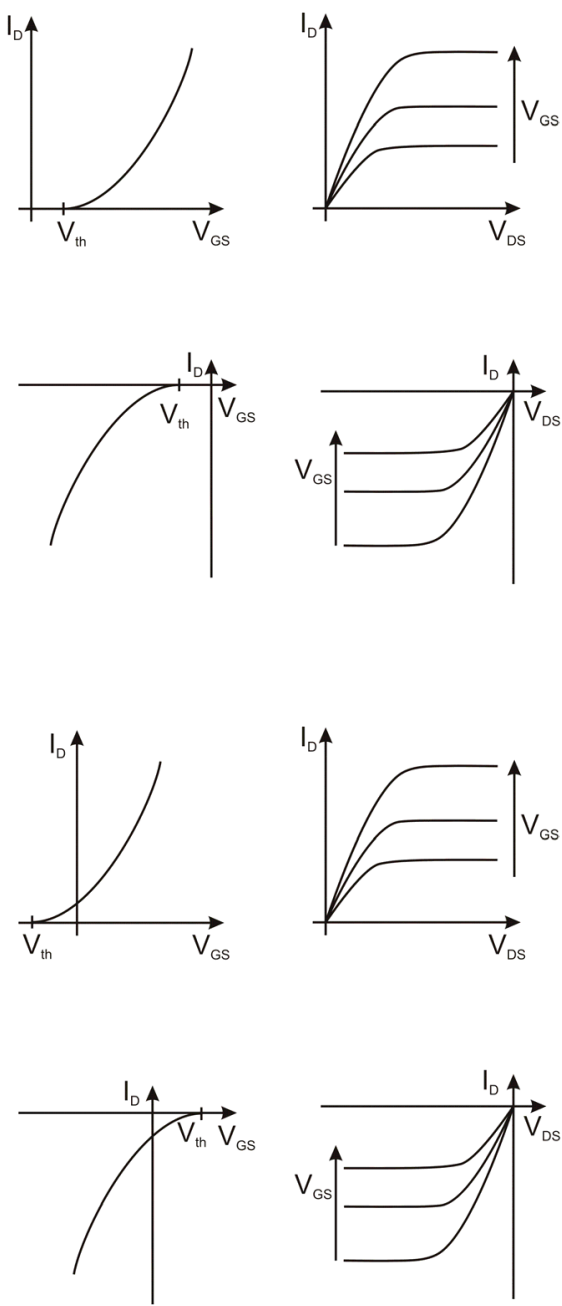

Fig. 3.8 Different types of field effect transistors (first column) with corresponding electrical symbol (second column), adopted from [66]. Third column $I_{D}$ vs. $V_{G S}$ and IV-curves (fourth column), adopted from [69]. 
Hence, the channel has the characteristics of a resistor with the conductance $g_{d}$ (in the limit as $\left.V_{D S} \rightarrow 0\right)$ [66].

$$
I_{D}=g_{d} \cdot V_{D S}
$$

This region is known as linear, ohmic, non-saturation, or triode region, cf. Fig. 3.9a.

The channel conductance is defined as [66]:

$$
g_{d}=\frac{W}{L} \cdot \mu_{n} \cdot\left|Q_{n}^{\prime}\right|
$$

with $\mu_{n}$ as the mobility of the charge carriers, here: electrons, in the inversion layer and the inversion layer charge per unit area $Q_{n}^{\prime}$. The inversion layer charge is a function of the gate bias and describes together with the material parameter $\left(\frac{W}{L}\right.$ and $\left.\mu_{n}\right)$ the slope of the IV-curve, i.e., $I_{D}$ vs. $V_{D S}$, in the linear region, cf. Fig. 3.9b. The current-voltage relationship in the linear region for an ideal ${ }^{6}$ MISFET is quantitatively given by (derivation of the equation can be found in [65]):

$$
I_{D}=\frac{W}{L} \cdot \mu_{n} \cdot C_{o x} \cdot\left[\left(V_{G S}-V_{t h}\right) \cdot V_{D S}-\left(\frac{1}{2}+\frac{\sqrt{\varepsilon_{S} \cdot q \cdot N_{A} / \psi_{B}}}{4 \cdot C_{o x}}\right) \cdot V_{D S}^{2}\right]
$$

for $V_{D S} \ll\left(V_{G S}-V_{t h}\right)$ Eq. (3.17) reduces to:

$$
I_{D}=\frac{W}{L} \cdot \mu_{n} \cdot C_{o x} \cdot\left(V_{G S}-V_{t h}\right) \cdot V_{D S}
$$

The device constants can be combined to the transconductance coefficient $K$ which describes the slope of the IV curve [69].

$$
K=\frac{W}{L} \cdot \mu_{n} \cdot C_{o x}
$$

\footnotetext{
${ }^{6}$ Ideal MISFET (after [65]) means that (1) the heart of the FET is an ideal MIS capacitor, (2) only drift current is considered, (3) carrier mobility in the inversion layer is constant, (4) doping in the channel region is uniform, (5) reverse leakage current is negligibly, and (6) the transverse field is larger than the longitudinal field.
} 
Equation (3.18) together with Eq. (3.19) leads to:

$$
I_{D}=K \cdot\left(V_{G S}-V_{t h}\right) \cdot V_{D S}
$$

with the threshold voltages $V_{t h}$ similar to an ideal MIS capacitor (see Section 3.1.1)

$$
V_{t h}=2 \cdot \psi_{B}+\frac{\sqrt{4 \cdot \varepsilon_{o x} \cdot q \cdot N_{A} \cdot \psi_{B}}}{C_{o x}}
$$
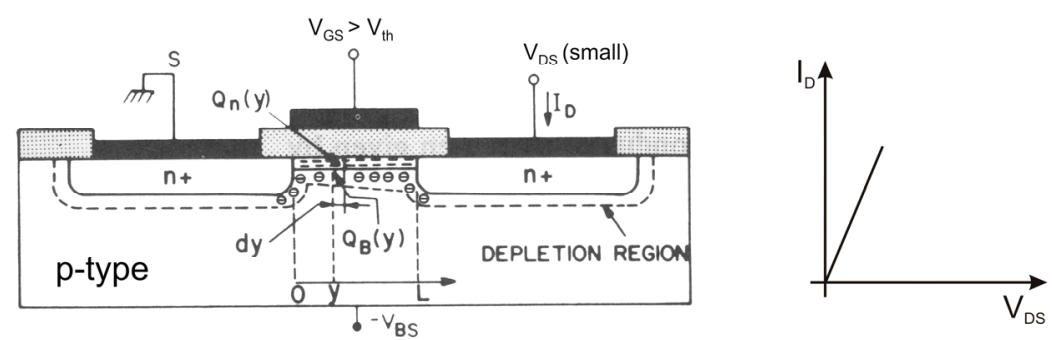

(a)
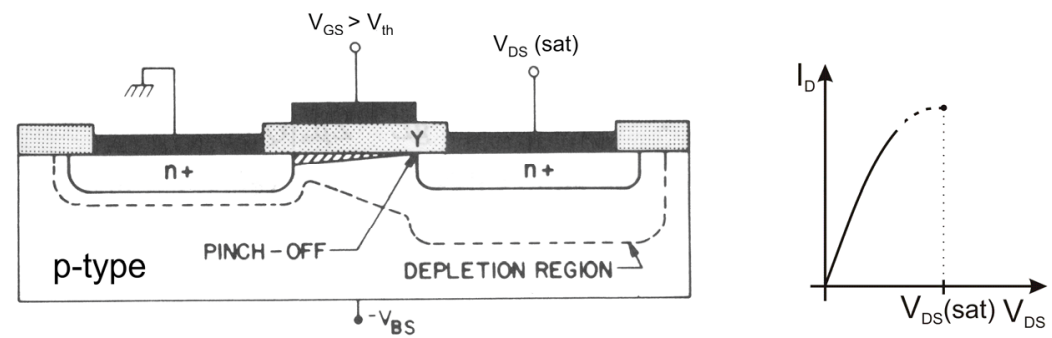

(b)

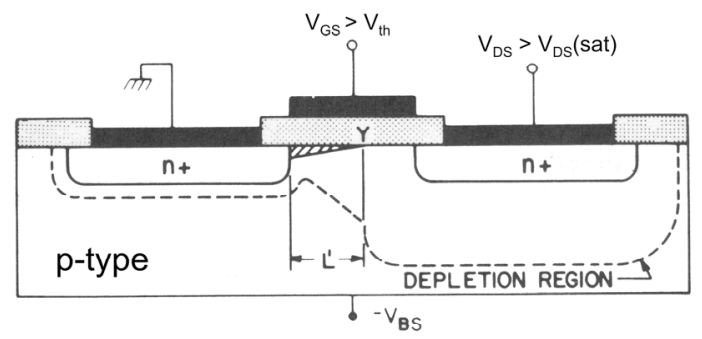

(c)

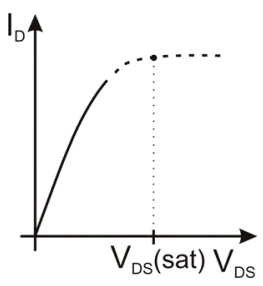

Fig. 3.9 Schematic of MISFET under different conditions (left, reproduced from [65] with kind permission from Wiley) and corresponding IV-curve (right). MISFET operated (a) in the linear region, (b) at the onset of saturation, and (c) beyond saturation. 
As the drain-source-voltage $V_{D S}$ increases further, the voltage drop across the insulator near the drain terminal decreases. Hence, the induced inversion charge density near the drain also decreases; respectively the channel depth near the drain terminal is reduced and the slope of the IV-curve will decrease, i.e., $g_{d}$ is lowered. At some point $V_{D S}$ might reach a point where the channel depth, i.e., thickness of the inversion channel, at the drain terminal is reduced to zero. This point is known as the pinch-off point and represents the onset of saturation, cf. Fig. 3.9b. Here, the potential drop across the insulator at the drain terminal is equal to the threshold voltage $V_{t h}$.

$$
V_{D S}(s a t)=V_{G S}-V_{t h}
$$

Beyond the pinch-off point the drain-source current $I_{D}$ remains constant (assuming that $\Delta L \ll L)$ although the channel length $L$ is reduced to $L^{\prime}$, resulting in a flat IVcurve, cf. Fig. 3.9c. This region is known as the saturation region. Electrons which are injected into the channel at the source terminal are injected to the space charge region at the point where the charge goes to zero, i.e., at the end of the channel, and are then swept by the electric field to the drain contact [66]. The current $I_{D}$ in that region is only modulated by the applied gate voltage, i.e., the gate-to-substrate field, i.e., the transverse field; hence, it is called Field Effect Transistor. The ideal current voltage relation in the saturation region is given by:

$$
\begin{gathered}
I_{D}=\frac{W}{L} \cdot \frac{1}{2} \cdot \mu_{n} \cdot C_{o x} \cdot\left(V_{G S}-V_{t h}\right)^{2} \\
I_{D}=\frac{K}{2} \cdot\left(V_{G S}-V_{t h}\right)^{2}
\end{gathered}
$$

An important parameter for FETs is the transconductance $g_{m}$ which is defined as the change in the drain current with respect to a change in the applied gate voltage [66]:

$$
g_{m}=\frac{\partial I_{D}}{\partial V_{G S}}
$$

For the linear region, the transconductance only depends on the drain-source voltage but not on the gate bias. 
The transconductance is always proportional to the transconductance coefficient $K$,

$$
g_{m L}=\frac{\partial I_{D}}{\partial V_{G S}}=\frac{W}{L} \cdot \mu_{n} \cdot C_{o x} \cdot V_{D S}=K \cdot V_{D S}
$$

and in the saturation region the transconductance is given by:

$$
g_{m S}=\frac{\partial I_{D}}{\partial V_{G S}}=\frac{W}{L} \cdot \mu_{n} \cdot C_{o x} \cdot\left(V_{G S}-V_{t h}\right)=K \cdot\left(V_{G S}-V_{t h}\right)
$$

\subsubsection{Non-ideal Effects}

In the previous discussion, an ideal MISFET was assumed. However the behavior of a non-ideal MIS capacitor should now be considered. Fixed oxide charges and the difference in work functions cause a voltage shift corresponding to the flat-band voltage $V_{F B}$, see also Section 3.1.1, Eq. (3.14), which itself changes the threshold voltage $V_{t h}[65]$ :

$$
\begin{aligned}
V_{t h} & =V_{F B}+2 \cdot \psi_{B}+\frac{\sqrt{4 \cdot \varepsilon_{o x} \cdot q \cdot N_{A} \cdot \psi_{B}}}{C_{o x}} \\
& =\left(\phi_{m s}-\frac{Q_{s}}{C_{o x}}\right)+2 \cdot \psi_{B}+\frac{\sqrt{4 \cdot \varepsilon_{o x} \cdot q \cdot N_{A} \cdot \psi_{B}}}{C_{o x}}
\end{aligned}
$$

When a substrate bias $V_{B S}$ is applied, source and substrate (body) do not have the same potential anymore causing electrons to flow laterally, from the source terminal to the substrate / body contact $\left(V_{B S}>0\right)$. For $V_{B S}>0$ more electrons are in the space charge region under the insulator, i.e., the width of the space charge region increases. Thus, the threshold voltage increases [66]:

$$
V_{t h}=V_{F B}+2 \cdot \psi_{B}+\frac{\sqrt{2 \cdot \varepsilon_{o x} \cdot q \cdot N_{A} \cdot\left(2 \cdot \psi_{B}+V_{B S}\right)}}{C_{o x}}
$$

The change in the threshold voltage can be written as [66]:

$$
\begin{aligned}
\Delta V_{t h} & =\frac{\sqrt{2 \cdot \varepsilon_{o x} \cdot q \cdot N_{A}}}{C_{o x}} \cdot\left(\sqrt{2 \cdot \psi_{B}+V_{B S}}-\sqrt{2 \cdot \psi_{B}}\right) \\
& =\gamma \cdot\left(\sqrt{2 \cdot \psi_{B}+V_{B S}}-\sqrt{2 \cdot \psi_{B}}\right)
\end{aligned}
$$


with the body-effect coefficient $\gamma$.

As shown in Fig. 3.9c, the length of the channel in saturation is reduced to $L^{\prime}$ which is due to the fact that the depletion region at the drain terminal extents laterally into the channel [66]. Since the depletion region is bias $V_{D S}$ dependent, the effective channel length also depends on the drain-source voltage $V_{D S}$. This effect is known as the channel length modulation. The reduced channel length $L^{\prime}$ affects the drain current $I_{D}^{\prime}$.

$$
I_{D}^{\prime}=\frac{L}{L-\Delta L} \cdot I_{D}=\frac{L}{L^{\prime}} \cdot I_{D}
$$

Since $L^{\prime}$ is a function of $V_{D S}$ also $I_{D}$ in saturation depends on $V_{D S}$

$$
I_{D}=\frac{K}{2} \cdot\left(V_{G S}-V_{t h}\right)^{2} \cdot\left(1+\lambda \cdot V_{D S}\right)
$$

where $\lambda$ is the channel length modulation parameter. This parameter can be derived from the IV-curve by extrapolating the curve for saturation. The corresponding voltage to the intercept of the extrapolated curve with the $V_{D S}$-axis is called Early voltage $V_{A}[69]$.

$$
\lambda=\frac{1}{V_{A}}
$$




\subsection{Gas Sensor Devices}

Several different kinds of field effect-based sensors as shown in Fig. 3.10 have been suggested as gas sensors.

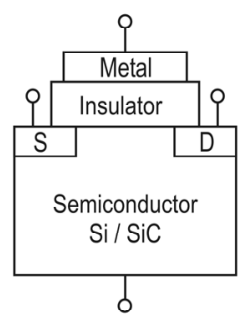

Fied Effect Transistor

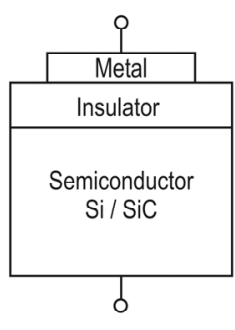

Capacitor

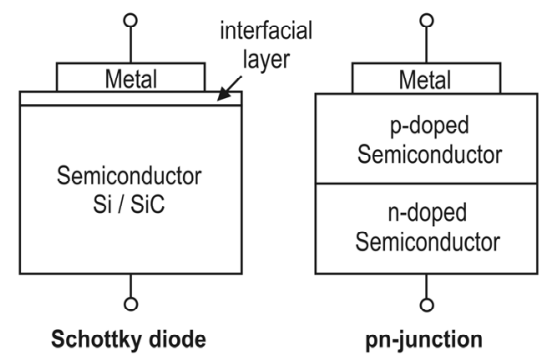

pn-junction

Fig. 3.10 Schematic diagram of different field effect devices. Adopted from [71].

Gas sensitive field effect transistors, cf. Section 3.2.1, capacitors as well as Schottky diodes consist in principle of a semiconductor substrate, usually but not necessarily silicon, $\mathrm{Si}$, or silicon carbide, $\mathrm{SiC}$, an insulator, e.g., silicon dioxide, $\mathrm{SiO}_{2}$, and a catalytic metal, e.g., palladium, platinum, or iridium. In literature, these devices are called MIS (metal insulator semiconductor) or, in case of a silicon substrate MOS (metal oxide semiconductor with respect to silicon) structures [65]. Besides the basic devices shown in Fig. 3.10, there are several variations, like suspended gate FETs, cf. Section 3.2.2, or nano-FETs, cf. Section 3.2.3, which do not follow the classic MIS structure.

\subsubsection{Field Effect Transistors}

Chemical sensors based on the field effect have been studied for more than 40 years by now. In 1970, Bergveld presented the first ion sensitive field effect transistor, ISFET, for pH-measurements [72] and the first gas sensor, a hydrogen-sensitive metal oxide semiconductor FET, MOSFET, was invented by Lundström in the mid1970s [73], [74], also known as Lundström-FET. Since then a lot of research has been done and developed this new class of chemical sensors to a main research area [75], [76]. Gas sensitivity of MOSFETs can be achieved by adding a catalytic 
gate metallization, e.g., palladium, platinum, or iridium to an ordinary MOSFET. The first devices were based on silicon, $\mathrm{Si}$, as a substrate material with a rather thick continuous palladium layer as gate contact which is in principle only sensitive to hydrogen or hydrogen containing gases forming hydrogen species by dissociation [76]. The sensing mechanism of gas sensitive FETs is discussed in detail in Section 3.4. However, the general idea is that a dipole layer is formed at the metalinsulator interface [77], [78]. This dipole layer acts as an additional, internal gate bias which alters the IV-curve of the transistor.

In the 1980s, several other FET sensors besides the hydrogen sensor have been suggested to detect among others ammonia, hydrocarbons, or carbon monoxide [76], [67]. Sensitivity to gases other than hydrogen arises from the gate morphology [79], in particular a thin, discontinuous gate metallization is required for detection of ammonia [80]. A detailed description of the sensing mechanism is given in Section 3.4.

Silicon as substrate material which was used in the beginning has the benefit to be processed in standard $\mathrm{CMOS}^{7}$ technology and thus, enabling the possibility of integrating the gas sensor and the readout electronics on one chip [81]. However, since the early 1990s silicon carbide, SiC, has been intensively studied and used as substrate material for gas sensors [82]. The first MIS gas sensors based on silicon carbide have been independently presented by two research groups: MIS capacitors at Linköping University, Sweden [83], [84] and Schottky diodes at NASA Glenn Research Centre in Cleveland, Ohio, USA [85]. Both types have disadvantages: capacitors are easy to fabricate but require a complex measurement setup and Schottky diodes suffer from poor long-term stability and reproducibility problems due to the extremely thin insulating layer. The first $\mathrm{SiC}$ field effect transistor as the ultimate field effect based transducer platform, was presented in 2000 by Savage [86], and further developed by Lloyd Spetz [82], [68]. In general, wide band gap materials like $\mathrm{SiC}$ which has a band gap of $3.2 \mathrm{eV}$ in case of $4 \mathrm{H} \mathrm{SiC}$, AlN with band gap of $6.3 \mathrm{eV}$, GaN with a band gab of $3.4 \mathrm{eV}$, or diamond with a band gap of $5.5 \mathrm{eV}$ [82], [87] are suitable materials for high temperature applications. Gas sensors based on $\mathrm{SiC}$ can typically be used up to $800^{\circ} \mathrm{C}$, however it was also reported that $\mathrm{SiC}$ capacitors can be operated up to $1,000{ }^{\circ} \mathrm{C}$ [88]. Furthermore, $\mathrm{SiC}$ is 
chemically inert which allows the sensor to be operated in harsh/corrosive atmospheres [82]. It was shown that the response time of SiC-FETs is below $100 \mathrm{~ms}$ for ammonia at $300{ }^{\circ} \mathrm{C}$, and below $20 \mathrm{~ms}$ for hydrogen at $550{ }^{\circ} \mathrm{C}$ [89]. Silicon carbide FETs have been applied to several industrial applications [90], for instance to combustion control in car exhaust [91], [92], [93], domestic boilers [94], [95], sulfur dioxide monitoring in power plants [96], [97], and monitoring ammonia slip in selective catalytic reduction, SCR, systems of diesel trucks [98]. Lately, SiC-FETs have been used to study the content of particles [99], [100].

SiC-FET gas sensors are commercial available by SenSiC AB, Kista, Sweden.

\subsubsection{Suspended Gate Field Effect Transistors}

Suspended Gate Field Effect Transistors, SG-FETs, have, in contrast to ordinary gas sensitive FETs, an air gap between the gate with its sensitive layer and the insulator of the FET structure as shown in Fig. 3.11. The first SG-FET device was suggested by Janata in the early-1980s [101] and have been further developed mainly by Eisele and co-workers [102], [103]. The idea of SG-FETs is to directly measure the work function change of the sensitive layer due to gas adsorption [104].
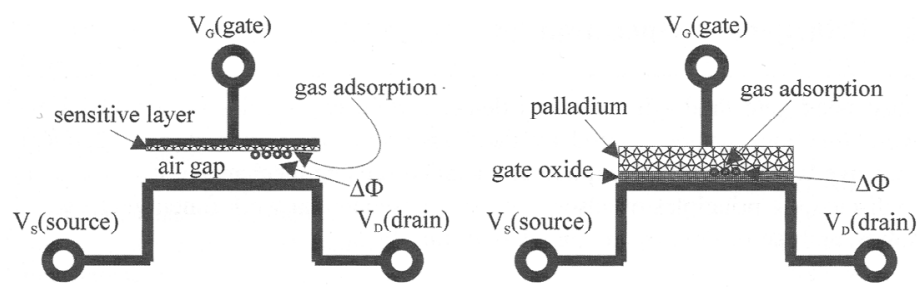

Fig. 3.11 (a) Schematic view of a suspended gate FET, and (b) a Lundström-FET. Reproduced from [105] with kind permission from Springer Science and Business Media.

The relation for the drain current from Eq. (3.18) becomes as follows [105]:

$$
I_{D}=\frac{W}{L} \cdot \mu_{n} \cdot C_{e f f} \cdot\left(V_{G S}+\frac{\Delta \phi}{q}-V_{t h}\right) \cdot V_{D S}
$$

with the effective capacitance from the air gap $C_{A}$ and the insulator capacitance $C_{I}$.

$$
C_{e f f}=C_{I} \cdot C_{A} /\left(C_{I}+C_{A}\right)
$$


The current state of the art SG-FET is the so called floating gate FET, FG-FET, [106] which is based on a capacitance-coupled FET, CC-FET [107]. The FG-FET consists of a capacitance-voltage-divider and a read-out FET as shown in Fig. 3.12.

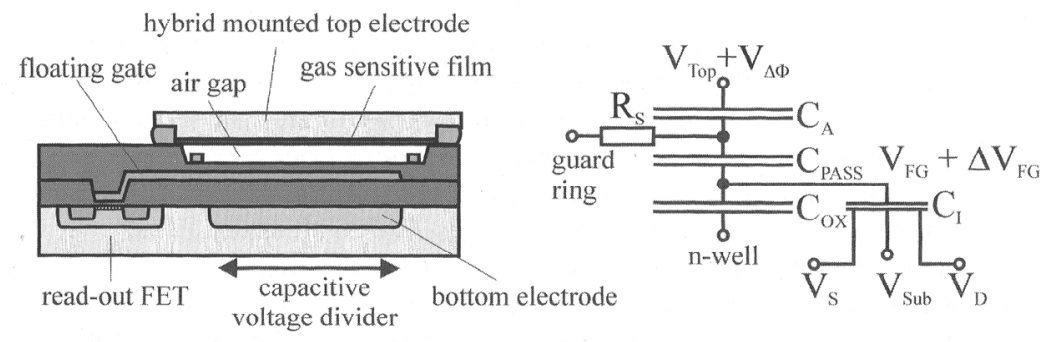

Fig. 3.12 (a) Schematic view, and (b) the equivalent electrical circuit of a floating gate FET. Reproduced from [105] with kind permission from Springer Science and Business Media.

The gate of the read-out FET is the large electrode, called the floating gate, which is located just opposite of the suspended gate underneath a passivation layer and which is connected to the middle electrode of the capacitance voltage divider. A change in work function of the sensitive layer alters the potential of the floating gate [105]:

$$
\Delta V_{G S}=\frac{C^{\prime}}{C^{\prime}+C_{o x}} \cdot \frac{\Delta \phi}{q}
$$

with effective capacitance $C^{\prime}$ from the gap $C_{A}$ and the capacitance of the passivation layer $C_{P a s s}$.

$$
C^{\prime}=C_{A} \cdot C_{\text {Pass }} /\left(C_{A}+C_{\text {Pass }}\right)
$$

The drain current of the read-out FET is then given as [105]:

$$
I_{D}=\frac{W_{e f f}}{L_{e f f}} \cdot \mu_{n} \cdot C_{I} \cdot\left(\frac{C^{\prime} \cdot V_{T o p}+C_{o x} \cdot V_{c a p}}{C^{\prime}+C_{o x}}+\Delta V_{G S}-V_{t h}\right) \cdot V_{D S}
$$

where $V_{T o p}$ is the applied bias to the top electrode (the sensitive layer) and $V_{\text {cap }}$ is the bias applied to the capacitance well on the backside of the device.

SG-FETs are commercialized by Micronas GmbH, Freiburg, Germany. 


\subsubsection{Nano-structured - Field Effect Transistors}

In the last years, a lot of effort has been spent on nano-structured sensitive layers [108], [109], [110] which are often used in combination with a field effect transistor as the transducer platform. This family of field effect devices is sometimes called nano-FETs. Normally, the nano-structure is deposited between the drain and source terminals of a field effect transistor. On the backside of the FET, a so called back-gate contact, which is similar to the substrate/body contact of an ordinary MISFET, can be used to modulate the conductance of the nano-structure [111] and influence the adsorption/desorption rate [112], [113]. Nano structures like nanowires, nanorods, e.g., $\mathrm{ZnO}$ nanorods, nanotubes, e.g., single and multi-wall carbon nanotubes, SWNT, MWNT, or graphene are promising candidates due to their high surface-to-volume ratio resulting in a high sensitivity [114]. The latter suffers from poor adhesion of the layer to a substrate making mounting and thus fabrication of a sensor device very challenging. Pearce et al. reported epitaxial growth of graphene one silicon carbide in order to overcome this issue and to build a graphene-FET [115], [68].

However, so far these kinds of field effect based gas sensors have not been used in commercially available mass products. Usually, these devices suffer from long time constants, lack of stability, and especially hysteresis effect [116]. 


\subsection{Surface Chemistry}

This section gives a brief overview of basic concepts regarding adsorption of gaseous substance on a solid, adsorption kinetics, and their influence on the work function.

\subsubsection{Physisorption, Chemisorption, and Dissociation}

Interaction of approaching gas molecules with the surface is the main cause for a sensor response. Usually, the gas molecules are adsorbed on the surface first and, depending on the circumstances, adsorption is followed by chemical reaction on the surface. Adsorption of a molecule in the gas phase on a solid surface is similar to the theory of chemical bonding and involves the same forces that are known from quantum mechanics [117]. Depending on the strength of the interaction between an adsorbant and the surface, different kinds of adsorption can be distinguished [118]:

- Weak interaction - physisorption

- Strong interaction - chemisorption

Physisorption is a process in which the electronic structure of the atom or molecule is hardly disturbed upon adsorption. The bonding is due to van der Waals forces which are weak interactions between (point) dipoles ${ }^{8}$ and / or induced dipoles [119]. Typical binding energies are 10-100 meV. Van der Waals forces have indeed a wideranging impact but are very weak. Adsorbed molecules are located typically 3-10 off the surface and are thus highly mobile in the plane of the surface. Usually, physisorption occurs at low temperature and is slightly exothermic with change in enthalpy (heat of adsorption) typically around $\Delta H_{a d s}^{p h y s} \approx-20 \mathrm{~kJ} \cdot \mathrm{mol}^{-1}$ [119].

Fig. 3.13 shows a schematic energy vs. distance diagram for a diatomic molecule $X_{2}$ approaching a metal surface (from the right-hand side of the diagram). If the molecule has a rather low energy, it first encounters only van der Waals forces and if the molecule loses energy upon interaction it may be trapped in the weak attractive potential [120]. If the molecule can overcome a small energy barrier, for instance by

\footnotetext{
${ }^{8}$ Point dipole is a dipole in which the distance between the centers of the two charges is small compared to the distance from which the dipole is observed.
} 
rearranging its electronic structure, it may become (associatively) chemisorbed. Chemisorption in contrast to physisorption involves real chemical bonds, usually covalent, which means overlapping of orbitals, i.e., sharing electrons or, ionic bonds which involve electronic charge transfer. Typical binding energies are 1-10 eV [118] at a distance of 1-3 $\AA$ from the surface [117]. Usually, chemisorption is exothermic with change in enthalpy (heat of adsorption) typically around $\Delta H_{\text {ads }}^{\text {chem }} \approx-200 \mathrm{~kJ} \mathrm{~mol}^{-1}[119]$.

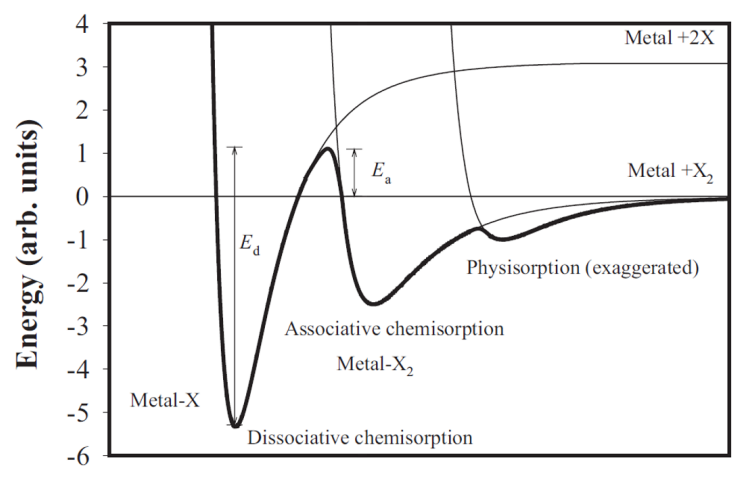

Reaction coordinate (arb. units)

Fig. 3.13 Schematic of the potential energy diagram along the reaction coordinate of a molecule $\mathrm{X}_{2}$ approaching a metal surface. Reproduced from [120] with permission from Wiley-VCH.

By moving the molecule $X_{2}$ closer to the surface, the energy rises due to a strong repulsive force. If it is able to overcome an energy barrier $E_{a}$, it may dissociate into two atoms [120] followed by adsorption (dissociative chemisorption). Adsorption is favored since it has a lower energy than dissociation in the gas phase (Metal $+2 \mathrm{X})$. In order to desorb an atom, the energy $E_{d}$ is needed. This is a very simplified picture and, in general, chemisorption depends not only on the temperature but also greatly on the surface and its structure.

\subsubsection{Adsorption Kinetics}

After introducing different kinds of adsorption, the kinetics behind adsorption should be discussed. At constant pressure and constant temperature there is an equilibrium between adsorption and desorption resulting in an equilibrium adsorbate 
coverage $\theta(p, T)$ which is described by the adsorption isotherm. If the surface is covered by a mono layer, the coverage is defined as one, $\theta(p, T)=1$. The simplest adsorption model is the Langmuir model which is based on the following assumption [118]:

- Adsorption is limited to a monolayer coverage

- All adsorption sites are equivalent, i.e., equally likely for adsorption

- Only one molecule can adsorb on an adsorption site and is not influenced by the neighboring sites

For non-dissociative adsorption the probability $\mathrm{f}(\theta)$ of finding an adsorption site is given by:

$$
\mathrm{f}(\theta)=1-\theta
$$

The ratio between the adsorption rate and the impinging rate, i.e., the flux of molecules impinging the surface, is defined as the sticking coefficient $s$ which depends on the adsorption probability $\mathrm{f}(\theta)$, the orientation and energy of the adsorbed molecules, also known as condensation coefficient $\sigma$, and the activation barrier for adsorption, i.e., the Boltzmann term.

$$
s=\sigma \cdot \mathrm{f}(\theta) \cdot \exp \left(-\frac{E_{a c t}}{k_{B} T}\right)
$$

The Langmuir kinetics is then given as following:

$$
\begin{aligned}
& \frac{d}{d t} \theta=s_{0} \cdot I \cdot(1-\theta) \\
& \Rightarrow r_{a d s}=p \cdot k_{a d s} \cdot(1-\theta)
\end{aligned}
$$

where $s_{0}$ is the sticking coefficient at zero coverage, $I$ the flux of impinging molecules, $p$ the partial pressure of a molecule and $k_{a d s}$ the rate constant of adsorption. The equilibrium coverage, the Langmuir isotherm, can be expressed as:

$$
\theta=\frac{K \cdot p}{1+K \cdot p}
$$

with $K=\frac{k_{a d s}}{k_{d e s}}$. 
In case of dissociative Langmuir adsorption which is usually the case for oxygen or hydrogen, the adsorption rate and coverage, i.e., the Langmuir isotherm for dissociative adsorption, changes as follows:

$$
\begin{gathered}
r_{a d s}=p \cdot k_{a d s} \cdot(1-\theta)^{2} \\
\theta=\frac{\sqrt{K \cdot p}}{1+\sqrt{K \cdot p}}
\end{gathered}
$$

The driving force behind adsorption is minimization of Gibb's free energy $G=H-T \cdot S$. One of the assumptions for the Langmuir adsorption model was that each adsorption site is energetically equal. However, in reality this assumption is sometimes violated and thus, the Langmuir model is no longer valid. In experiments, the adsorption enthalpy often increases with increasing coverage. One approach to compensate for this effect is the so called Temkin isotherm which states that the adsorption enthalpy depends linearly on the pressure $p$ [119].

$$
\theta=c_{1} \cdot \ln \left(c_{2} \cdot p\right)
$$

where $c_{1}$ and $c_{2}$ are empirically determined constants.

If the sticking probability remains non-zero after completion of the first monolayer, Langmuir and Temkin isotherms are not valid. For multilayer adsorption, the $B E T$-isotherm ${ }^{9}$ has to be used [118], [121].

\subsubsection{Workfunction Change by Adsorbates}

Adsorbed species on a surface generally have a significant influence on the electronic structure of the surface that can be expressed by a change in work function. As discussed in Section 3.3.1, there are basically two types of adsorption: Physisorption which is a weak interaction between the gaseous substance and a surface, and chemisorption which changes the electronic structure of a surface. Physisorption typically results in a dipole layer due to the formation of an image charge just below the surface. Chemisorption involves charge transfer from the

\footnotetext{
${ }^{9}$ Suggested by Brunauer, Emmett, and Teller in 1938.
} 
substrate to the adsorbate or vice versa resulting in surface filled states [117], [122]. This gives rise to additional dipole moments. The magnitude of the dipole layer depends on the ionic property of the chemical bond which is related to the difference in electro negativity between the bonding partners [123].

As shown in Fig. 3.14, both surface states, SS, and a dipole layer, DL, on a semiconductor surface change the semiconductor work function. Surface acceptors, cf. Fig. 3.14b, capture electrons from the conduction band until they are completely filled resulting in a band bending [122]. This can be expressed by a change in electron affinity $\chi$ :

$$
\chi_{S S}=\chi_{0}+\left(-e V_{S}\right)
$$

where $\chi_{0}$ is the electron affinity without surface states. The second term describes the additional band bending due to surface states.

For a dipole layer, cf. Fig. 3.14c, the electron affinity is changed as, after [117], [68]:

$$
\chi_{D L}=\chi_{0}+\frac{N \cdot p}{\epsilon_{0}}
$$

where $N$ is the density of dipoles with a dipole moment $p$ on the surface and $\epsilon_{0}$ the permittivity in free space. In fact, the permittivity is also affected by the insulator. Thus, it is rather $\epsilon_{0}{ }^{\prime}$ than $\epsilon_{0}$.

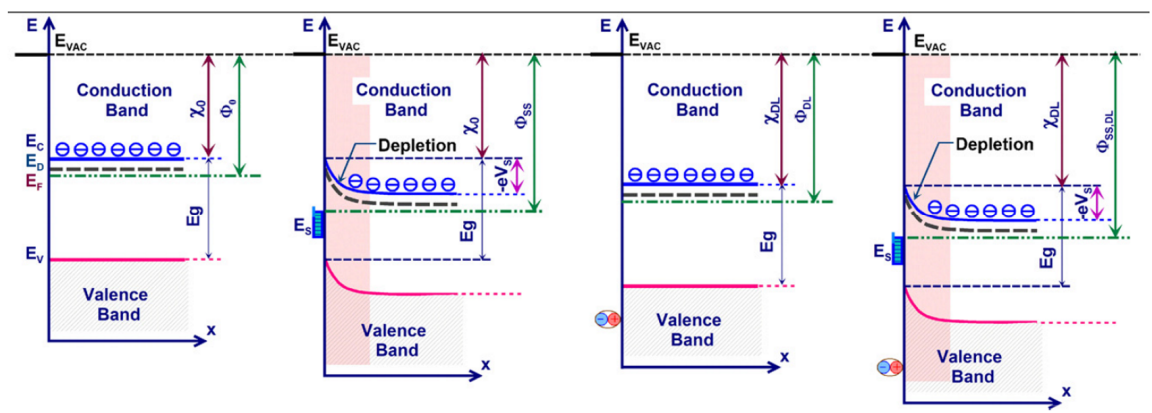

Fig. 3.14 Influence of adsorbates on the work function of an n-type semiconductor. (a) Before adsorption, (b) with surface states, SS, (c) with a dipole layer, DL, and (d) with both SS and DL. Reproduced from [122] with kind permission from Elsevier. 
In case of a MIS-structure, the difference in metal-semiconductor work function, Eq. (3.2) changes into:

$$
\phi_{m s}=\phi_{m}-\left(\chi+\Delta \chi+\frac{E_{g}}{2 q}+\psi_{B}\right)
$$

Strictly speaking, also the metal work function can be affected, in particular if gaseous substances are adsorbed directly on the gate metal.

As mentioned earlier, chemisorption causes charge transfer and thus, additional charge can be located on or in the insulator. In Section 3.1.2.2, the following equation, Eq. (3.28), was derived for the threshold voltage of a MISFET:

$$
\mathrm{V}_{\mathrm{th}}=\left(\phi_{m s}-\frac{Q_{s}}{C_{o x}}\right)+2 \cdot \psi_{B}+\frac{\sqrt{4 \varepsilon_{o x} \cdot q \cdot N_{A} \cdot \psi_{B}}}{C_{o x}}
$$

The first term $\left(\phi_{m s}-\frac{Q_{s}}{C_{o x}}\right)$ was earlier defined as the flat band voltage of the transistor and depends not only on the metal-semiconductor work function but also on the charge $Q_{s}$ located in the insulator. Thus, any charge transfer from an adsorbate to or from the insulator causes a shift in the threshold voltage of the transistor.

In summary, any gas interaction with the gate surface of a MISFET leading to a change in the threshold voltage can be seen as an additional, internal gate bias $V_{G S_{\text {internal }}}$ as already mentioned in Section 3.2.1. Consequently the IV-characteristic of a MISFET from Eq. (3.24) changes to:

$$
I_{D}=\frac{K}{2} \cdot\left(V_{G S_{\text {external }}}+V_{G S_{\text {internal }}}-V_{t h}\right)^{2}
$$




\subsection{Sensing Mechanisms}

This section deals with the sensing mechanism of gas sensitive field effect transistors. The derivations given in the following are mainly based on book chapters by Andersson [68] and Lloyd Spetz [90], [71].

\subsubsection{General}

The IV-characteristic of a MISFET depends, as discussed earlier, on parameters such as device dimensions and semiconductor properties, e.g., electron mobility, permittivity, doping, etc. However, as seen from Eq. (3.28), charges located in or on the insulator $Q_{S}$ as well as the change in metal-semiconductor work function $\phi_{m s}$ influences the threshold voltage $V_{t h}$ and therefore the drain-source current of the transistor, cf. Eq. (3.23). These effects can be seen as an internal gate bias in addition to the externally applied bias to the gate contact, cf. Eq. (3.50) [68]. Consequently, any gas interaction which leads to the formation of an internal gate bias by charge injection or transfer, or any change in work function alters the IV-characteristic of the FET device - the sensor.

\subsubsection{Hydrogen-Containing Gases}

The first gas sensitive FET was presented by Lundström as a hydrogen sensor with a dense catalytic gate metallization [73]. The sensing mechanism of FET devices is probably best understood for hydrogen, $\mathrm{H}_{2}$, and hydrogen containing gases, e.g., hydrocarbons, $\mathrm{HC}$, and ammonia, $\mathrm{NH}_{3}$.

First, a thick, dense metal film on the gate is considered, cf. Fig. 3.15a. Hydrogen dissociatively adsorbs on metal surfaces such as palladium, Pd, platinum, Pt, or iridium, Ir. Then, hydrogen species, mainly protons, diffuse rapidly, in the order of $10^{-5} \mathrm{~s}$, through the metal to the metal-insulator interface. The ratio of hydrogen surface coverage and interfacial hydrogen are in equilibrium if only hydrogen is present. The surface coverage follows a typical Langmuir relation and depends only on the ambient hydrogen pressure [124]. For oxidic insulators, the hydrogen atoms 
adsorb to oxygen atoms on the surface of the insulator forming hydroxyl, $\mathrm{OH}$, groups, i.e., a dipole layer at the metal-insulator interface [77], [78] which themselves cause charge transfer in the semiconductor. At this interface, the adsorption shows more of a Temkin behavior [125], [126], [127] because of dipoledipole interaction. Due to the strong dipole moment of the OH-groups, the electric field in the insulator is changed thus affecting the drain-source current $I_{D}$ of the device [128]. The effect of a dipole layer at the metal-insulator interface has earlier been described as an additional, internal gate bias.
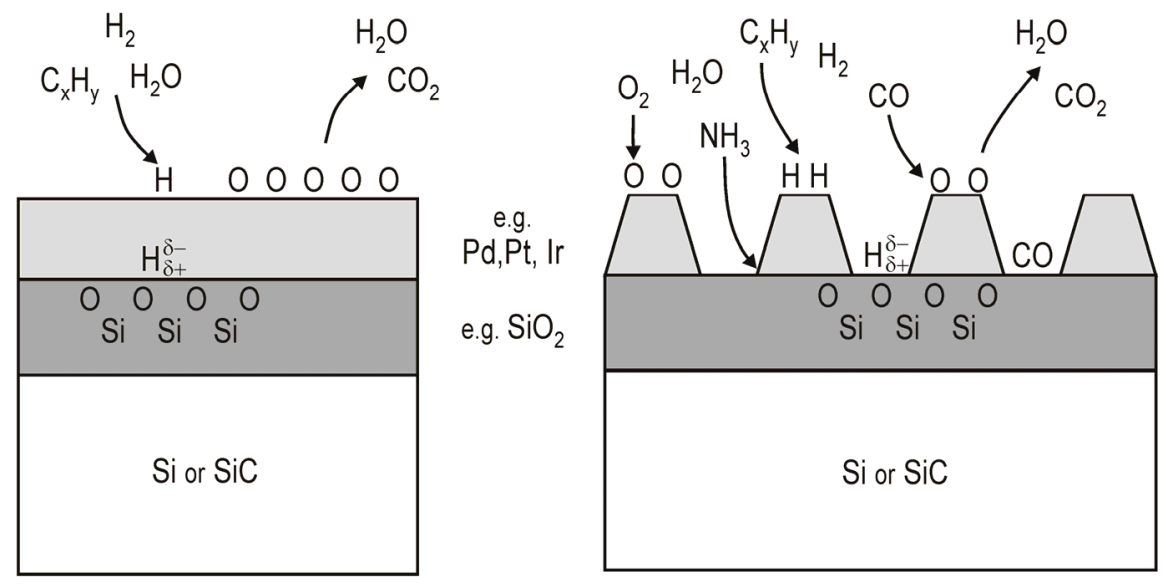

Fig. 3.15 Schematic illustration of the sensing mechanism of a FET gas sensor (a) with a dense catalytic gate, and (b) with a porous gate. Adapted from [68].

Not only hydrogen dissociates on a metal surface and (partly) covers the surface but also other substances which can hinder the adsorption of hydrogen. For instance, oxygen also dissociatively adsorbs on common gate metals [68]. In an oxygen rich atmosphere, hydrogen molecules approaching the surface react to some extent with adsorbed oxygen to water and leave the surface without diffusion and creation of a dipole layer at the interface. The other hydrogen atoms diffuse through the metal forming the $\mathrm{OH}$-dipole layer. If the hydrogen supply is turned off, the hydrogen atoms diffuse backwards and react with the oxygen adsorbed on the metal surface [71]. It was reported that the formation of a dipole layer is the dominating effect in terms of sensing mechanism. Adsorption of hydrogen to the metal at the interface causes a change in the work function of the metal, but the effect is rather small and in fact, leads to a shift in the IV-curve in the opposite direction [77]. 
Therefore, the number of oxidic sites of the insulator is the key parameter of the sensor as proven in studies by Schalwig [129], Weidemann [130], and Eriksson [131] where different insulator materials have been tested. For other hydrogen containing gases basically the same principle applies as for hydrogen if free hydrogen species can be generated by dissociation. For the dissociation process of hydrocarbons the temperature is crucial. However, no sensor response is obtained for ammonia with a thick, dense gate metal or for saturated hydrocarbons in atmospheres with excess oxygen [132], [133]. The dissociation of hydrogen can be hindered by the presence of other gases, e.g., carbon monoxide, even if the sensor itself does not show any sensitivity to the interferent [134].

In order to detect ammonia, a porous or discontinuous gate is required [79], [80], cf. Fig. 3.15. In case of ammonia detection, it is commonly accepted that three phase boundaries, where oxide, metal and the gas phase meet, are needed in order to dissociate ammonia such that hydrogen atoms or ions are created. The hydrogen atoms / ions formed at the three phase boundaries either diffuse out onto the insulator surface and forms OH groups [135], or the hydrogen may diffuse back on the metal surface and form water with adsorbed oxygen. Also the detection of ammonia is reversible since the $\mathrm{OH}$-groups decompose again and hydrogen forms water with oxygen most likely at the metal surface [71].

In oxygen containing atmospheres, oxygen can dissociatively adsorb on metal surfaces. It was reported that oxygen ions can spill over from the metal to bare patches of oxide [136], [137]. These negatively charged ions (anions) themselves create an electric field which influences the mobile carriers in the channel of the transistor underneath. Schalwig et al. [136] have suggested that the sensing principle for hydrogen and non-hydrogen containing gases (for details see next sub-section) has a common cause. Oxygen anions can reverse spill over from the oxide to the metal and/or be removed through reaction with, e.g., hydrogen or other gases approaching the surface of the sensor. The removal of negatively charged ions from the oxide surface affects the IV-curve in the same way as the voltage drop by $\mathrm{OH}$ group formation [68].

At this point, a brief comparison of a FET and a suspended-gate FET (cf. Section 3.2.2) will be given. For FETs with a porous gate, spill-over and reverse spill-over effects take place as described earlier and especially the formation of a 
dipole layer is the dominating sensing effect. However, for SG-FETs where the sensing layer is uncoupled ("suspended") from the oxide and the rest of the transistor, these spill-over effects do not occur due to the air gap which is in the range of a few micrometers. In this case, the influence on the semiconductor, and therefore on the read-out transistor, is only based on work function changes due to gas adsorption. Gas interaction with the sensing (suspended) electrode leads to charge transfer to or from the suspended gate. Hence, the response of a SG-FET is usually lower than for a FET [90].

\subsubsection{Non-Hydrogen-Containing Gases}

Detection of non-hydrogen containing gases is less well understood. Nevertheless, a porous or discontinuous gate is required to achieve sensitivity. In case of carbon monoxide, $\mathrm{CO}$, some possible explanations have been suggested in literature. Firstly, molecular dipoles, e.g., $\mathrm{CO}^{10}$ can directly adsorb on bare patches of oxide. This polarized layer creates an electric field which influences the mobile carriers in the channel of the transistor [135]. Earlier studies suggest that $\mathrm{CO}$ adsorbs directly on metal clusters and reversible reduces / oxidizes surface metal oxide [138], [139]. This work function change close to the metal oxide border can be detected by capacitive coupling through the pores of the discontinuous film [140], [67]. As already mentioned for hydrogen containing gases, oxygen, $\mathrm{O}_{2}$, plays an important role. It was suggested that oxygen ions can spill over from the metal to the oxide. The negatively charged oxygen anions change the IV-characteristics of the device. In presence of reducing gases such as $\mathrm{CO}$, surface reactions will take place and thus, lower the amount of oxygen on the surface [136].

For $\mathrm{CO}$ detection especially the ratio between $\mathrm{CO}$ and $\mathrm{O}_{2}$ on the surface is crucial. It was reported that $\mathrm{CO}$ exhibits a binary response, i.e., sharply switching between small and large. The sensor response correlates well with the $\mathrm{CO}$ coverage on the surface [139]. For an increase in the $\mathrm{CO} / \mathrm{O}_{2}$ ratio or a decrease in temperature, the response switches from a small to a large response [139], [138]. The transition between $\mathrm{CO}$ coverage and oxygen domination on the surface is strongly temperature depended [141]. For a fully CO covered Pt surface, thus oxygen depleted surface, the

\footnotetext{
${ }^{10}$ Carbon monoxide has a small dipole moment which is due to the three covalent bonds.
} 
$\mathrm{CO}$ oxidation rate, i.e., production of carbon dioxide, $\mathrm{CO}_{2}$, on, e.g., platinum is reduced, and the oxidation reaction is self-poisoned leading to a large response. In contrast, an oxygen dominated surface exhibits a high oxidization rate, i.e., $\mathrm{CO}_{2}$ production, but with a very small sensor response [96]. In fact, oxidation of CO on catalytic metal films is a competing reaction. Depending on temperature, gate morphology, $\mathrm{CO}$ and oxygen concentration oscillation in the sensor response can be observed [142], [143], [144], [145]. Since the oscillatory kinetics of $\mathrm{CO} / \mathrm{O}_{2}$ is very sensitive changes in the composition of the gas phase, it can be used to study surface reactions [146].

Besides the gate material and its morphology, the insulator plays a major role as well. It was reported that by exchanging the commonly used silica, $\mathrm{SiO}_{2}$, by other insulating materials, SiC-FETs can be tailored to specific applications [147], [148], [149]. For instance when using magnesium oxide, $\mathrm{MgO}$, the cross-sensitivity to hydrogen containing gases is reduced and also the $\mathrm{CO}$ sensitivity is lowered making SiC-FETs sensitive to oxygen [147]. 


\subsection{Terminology Related to Gas Sensors}

In this section, definitions of commonly used terms related to gas sensors are given since terminology is not used consistently. Equations are exemplarily given for gas sensitive field effect transistors operated in a constant current mode ( $I_{D}=$ const.), i.e., the voltage drop between drain and source $V_{D S}$ is measured.

Sensor signal is the output signal of the transducer, also known as sensor raw data. For gas sensitive field effect transistors the sensor signal is often the drain-source voltage $V_{D S}$ at a constant drain-source current $I_{D}$.

Response $\Delta V_{D S}$ which is sometimes called differential signal is the change of the sensor signal towards a target gas compared to the sensor signal in the carrier gas, e.g., pure air.

$$
\Delta V_{D S}=V_{D S}(\text { gas })-V_{D S}(\text { air })
$$

Relative signal $V_{D S}^{\mathrm{r}}$ is the relative change of the sensor signal towards a target gas. The relative change of resistance or conductance is widely used for metal oxide semiconductor gas sensors [123].

$$
V_{D S}^{\mathrm{r}}=\frac{V_{D S}(\text { gas })}{V_{D S}(\text { air })}
$$

Relative response or fractional signal is the relative change of the sensor response towards a target gas. It is also commonly used for describing the response of a gas sensor [123].

$$
\Delta V_{D S}^{\mathrm{r}}=\frac{V_{D S}(\text { gas })-V_{D S}(\text { air })}{V_{D S}(\text { air })}=\frac{\Delta V_{D S}}{V_{D S}(\text { air })}
$$

The equations given above defining sensor response, relative signal and relative response can also be used for baseline manipulation in order to reduce the influence of sensor drift [150], cf. Section 5.1. 
Response curve, calibration curve, or calibration function is the non-statistical relationship for a chemical measurement process, the observed signal, response or relative signal/ response to the analyte amount [151]. It can be derived from the sensor response, relative signal or relative response in steady-state as a function of the input concentration of the analyte [123]. The sensor response curve is repeatedly but improperly called sensitivity curve [123], [152].

Baseline is the response at zero concentration of a target gas, i.e., just in carrier gas.

Sensitivity $S$ is the slope of the calibration curve [151]. Gas sensors usually have a non-linear calibration curve, thus sensitivity is a function of the concentration $c$. Due to the fact that the calibration curve, i.e., response curve, can be defined in various ways (see definitions above), there are several different definitions for sensitivity, however, it is always the derivation of the output signal with respect to the analyte amount [152]. The analyte amount is usually given by the concentration but also partial pressure of the analyte is appropriate [153], [154].

$$
\mathrm{S}(\mathrm{c})=\frac{\partial \text { calibration curve }}{\partial \text { analyte amount }}
$$

Selectivity in a qualitative sense describes the extent to which the sensor responds to other gases of interest for the given procedure/ environment. The term selectivity according to IUPAC can further be used for quantitative purposes in conjunction with another metric, e.g., constant, coefficient, index, factor, number, for quantitative characterization of interferences [151]. A fully-selective analytical method allows determination of a substance without any sensitivity to other substance [155]. This method or sensor is then specific for a certain analyte. Generally, there is no specific chemical gas sensor so far; however, selectivity can greatly be increased by advanced sensor operation modes (for an overview of state of the art operation modes see Section 3.5).

Cross sensitivity describes the sensitivity of a sensor towards interferences, i.e., to which extent the interference influences the sensor response.

Reproducibility is defined by IUPAC [151] as "the closeness of agreement between independent results obtained with the same method on identical test materials but under different conditions." 
Repeatability in contrast to reproducibility is defined as "the closeness of agreement between independent results obtained with the same method on identical test material, under the same conditions (same operator, same apparatus, same laboratory and after short intervals of time)" [151].

Stability can be defined as the repeatability over extended periods of time, i.e., the signal of the sensor remains almost constant under the same conditions over a long period of time. Frequently, stability is also called long-term stability in order to highlight the repeatability over an extensive period of time.

Drift is a slow non-random change in the signal / of the baseline with time [151].

Resolution describes the smallest change in quantity being measured that can be distinguished with $95 \%$ probability [151], [156].

Limit of detection or detection limit is the concentration or quantity that can be detected with a reasonable certainty [151], i.e., with a reasonable signal-to-noise ratio, SNR. Typically, three times the standard deviation ( $3 \sigma$-criterion) is chosen to define the detection limit.

Poison in catalysis refers to an inhibitory substance characterized by its propensity to attach very strongly, i.e., by a true chemical bond, to the surface blocking the catalytically active sites [151]. In chemical gas sensing, one distinguishes between reversible sensor poisoning and irreversible sensor poisoning. Reversible poisoning means that the blocking of chemically active sites can completely be removed by a drastic change in operating conditions, e.g., temperature. An example is carbon monoxide poisoning. Irreversible poisoning, e.g., by sulfur or silicone species on the sensing layer, cannot be removed by just heating up. In that case, the sensor usually needs to be replaced. Self-poisoning takes place in chemical reactions in which a product cause poisoning or inhibition [151]. An example for this is the oscillating reaction of carbon monoxide on platinum. 


\section{Advanced Sensor Operation}

Solid state gas sensors like resistive type metal oxide, MOX, or field effect, FE, based gas sensors usually offer high sensitivity but suffer from poor selectivity. Long time stability is also a crucial issue. Due to that, solid state gas sensors are often not the first choice for safety-critical applications. Nevertheless, the performance of such sensors can significantly be enhanced by appropriate operation.

\subsection{Sensor Arrays}

A common way to overcome the lack of selectivity are sensor arrays which were first suggested in 1982 by Persaud and Dodd [157] and which have become popular in the 1990s and early 2000s as the heart of so called electronic noses, EN, [158], [159], [18], [160]. The reaction of chemical sensors strongly depends on surface reaction and catalysis, operating temperature, and the choice of the catalytic material are key parameters. The idea of a sensor array is to use several different kinds of sensors and / or sensors at different temperatures. The output of such an array is a huge dataset containing an ideally unique fingerprint for each odor or, in general, for each gas exposure. In the past decades, various sensor principles have been used in ENs, for instance MOX sensors, MOSFETs [161], surface acoustic waves, SAW, quartzcrystal microbalances, QMB, as well as conducting polymers, $\mathrm{CP}$, optical sensors, electrochemical sensors, EC, and any combination of those [158]. In the last years, nano-materials have been intensively used to setup sensor arrays with up to several hundred sensing elements [162]. Besides chemical sensors also new approaches with, e.g., optical sensors or different spectroscopy techniques have been suggested [163]. Disadvantages of sensor arrays are in principle the lack of stability and the complexity of the entire sensing element. The lack of stability arises mainly from different drift behaviors of different sensors and thus, a huge effort for recalibration. 


\subsection{Temperature Modulation}

Another approach to enhance the performance of a chemical sensor is transient operation. In contrast to constantly operated sensors or classical sensor arrays, transient operation offers several advantages. The most well-known technique is temperature modulation and was first reported by Eicker [164] who used a varying heater voltage covering a broad temperature range. Temperature modulation gives rise to several advantages, as shown by Lee et al. [165] and Sears et al. [166], [167]: A broad temperature range covers usually points of maximum sensitivity of target analyte, hence the sensitivity is enhanced. For some gases like carbon monoxide high sensitivity is achieved at low temperatures, which often causes accumulation of incompletely oxidized components on the sensor surface. Periodic heating of the sensor up to high temperatures, typically in the order of several hundred degrees, can "clean" the surface and thus, increase the stability of the system. Due to the fact that the sensor is never in steady state, the transient response results in an ideally unique pattern for each gas, thereby increasing the selectivity. A more recent review on temperature-modulated gas sensors can be found in [168].

Additionally, it was also reported that transient operation leads to faster gas detection than constantly heated sensors [169], [170]. Several different ways of stimulating a sensor have been proposed and two classes can be distinguished [171]: 1) discrete temperature modulation, e.g., temperature pulses [172], [173], steps or stairs [174], and 2) continuous temperature modulation like temperature ramps, sinusoidal heating of MOX sensors as suggested by Nakata et al. [175], [176], [177], or modulating frequencies [178]. Heater pulses in particular lead to a significant reduction of power consumption [179], [174] which is important for wireless, battery driven systems. For sensors operated by a pulse or step waveform, the discriminatory information is mainly contained in the chemical transient after the heater voltage is switched off [180], [181], [182]. Semancik et al. suggested using a series of heater pulses with different heights. In this temperature-programmed sensing, TPS, the temperature after each pulse always goes back to a base-temperature. The various pulse heights cause different transients creating a unique finger print [183], [184]. Lately, 
Semancik and co-workers used microsensor arrays where each sensor is operated in TPS in order to increase the selectivity even further [185], [186].

Transient parameter extraction from the sensor response of a single MOSFET was used for simultaneous prediction of hydrogen and ethanol [187]. Further, it was reported that a modulated operating temperature can be used to reduce the recovery time of a MOSFET as well as allow gas discrimination [188].

A non-stationary temperature-pulse mode has been suggested where high operating temperatures for silicon based field effect devices have been used [189], [190]. Since silicon has a small band gap, typical constant operating temperatures are limited to $200{ }^{\circ} \mathrm{C}$. However, in order to detect, e.g., hydrocarbons higher temperatures are needed. To overcome this dilemma, temperature cycled operation, TCO, has been used where each cycle consists of a high and low temperature phase. At the high temperature, the chemical reactions and interactions with the sensor surface happen. This situation can successfully be "frozen in" by a rapid drop in operating temperature. During the low temperature phase the electrical read out is done [140].

There are several publications dealing with adaptation of the optimal operating temperature [191], [192]. The adaptation may take place at the hardware level and at the data analysis level as described in a review by Gutierrez-Osuna [193]. 


\subsection{Bias Modulation}

Temperature modulation is possible for basically all kinds of solid state gas sensors but was originally developed for resistive type metal oxide gas sensors. However, not only temperature modulation can be used for transient characterization but, in case of FETs, also small changes in bias voltage [194]. In particular field effect based gas sensors offer the possibility to change and modulate the applied bias. For suspended gate field effect transistors it was reported that the sensing performance of a humidity sensing FET can be enhanced by pulsed operation of the voltage applied to the suspended gate [195]. With the transient readout a much higher correlation between the sensor response and the actual value of humidity was achieved. Further, the cross-sensitivity to hydrogen, nitrogen dioxide, and ammonia was improved [196]. Wöllenstein et al. studied the effect of a pulsed gate bias on insulated gate thin-film transistors. It was reported that a positive bias increases the sensor response to ammonia, whereas a negative bias increases the response to nitrogen dioxide [197].

In the area of gas sensing with nano-structures on a transistor platform, strong hysteresis effects have been found when sweeping the (back) gate bias. Kim et al. reported that the hysteresis in the $I_{D}-V_{G S}$-curve is due to adsorbed water molecules on the nanotube which induce charge traps [198]. In general, the hysteresis found in carbon nano-tube FETs, CNT-FET, is due to charge trapping which is caused by either adsorbed species on the CNT or by charge injection from the CNT into the underlying oxide [116], [199]. Filled charge traps at the insulatorsemiconductor interface or in the insulator but close to the surface create an additional potential on the gate. Changing the externally applied gate bias leads to trapping and de-trapping of charge which is usually a slow process [200]. Hence, the additional potential caused by charge traps leads to a pronounced hysteresis effect when sweeping the gate bias $V_{G S}$. Usually hysteresis is unwanted since it leads to memory effects. Several strategies have been proposed to reduce hysteresis. Mainly coating of the CNT can help to avoid adsorption of molecules; however for gas sensing encapsulating the nanotube is not feasible. Mattmann et al. suggested using short gate bias pulses instead of a continuous gate bias ramp [201]. During a short pulse there is not sufficient time for the filling of charge traps. Hence, hysteresis 
could be reduced by $20 \%$ and with ambipolar pulses, which means a short positive pulse followed by a short negative pulse, the hysteresis can be reduced almost completely [202].

Nevertheless, hysteresis depends also on the gas exposure [200], [203]. Chang et al. reported that gate pulses with different polarity change the adsorption-desorption behavior of the nanotube [203]. A negative bias increases desorption rate of nitrogen dioxide whereas a positive bias desorbs ammonia. It was reported further, that different gate pulses shorten the recovery time after gas exposure considerably.

The effect of an applied bias on surface chemistry, like adsorption of oxygen ions on metal surfaces, was also found for yttria-stabilized zirconia, YSZ, for oxygen sensors [204], [196]. The authors suggest that the applied bias alters the reaction of chemisorbed oxygen ions on platinum, i.e., the formation of platinum oxide. 


\subsection{Virtual Multi-Sensor}

As discussed in the previous sections, dynamic operation can significantly enhance the performance in terms of selectivity, stability, and speed of chemical sensors. In distinction to sensor arrays, the term virtual multi-sensor has been introduced for a single sensor with a modulated operating temperature [19] since the sensor shows similar characteristics as an array [205]. If the temperature is modulated in a cycled way, in contrast to pulsed variations, the term temperature cycled operation, TCO, is often used [205]. Temperature cycled operation requires, similar to sensor arrays, more demanding evaluation strategies compared to single static operated sensors. Thus, the virtual multi-sensor approach not only means using a single sensor in TCO but also the necessary data evaluation and signal processing steps. Typically, pattern recognition techniques based on multivariate statistics are used, cf. Chapter 5.

At the Lab for Measurement Technology, Saarland University, Germany, TCO has been studied and successfully applied to various applications, like solvent detection, underground fire detection, indoor fire detection, odor assessment, among others, for almost 15 years [171], [206], [7], [207], [8]. Mainly thick film resistive type metal oxide sensors [8] or pellistors [208] as well as micro structured MOX sensors [7] have been used. Besides TCO, electrical impedance spectroscopy, EIS has lately been used as an alternative [209], [210] as well as in combination with TCO for selfmonitoring of the sensor [211], [212], [213].

Temperature cycled operation in combination with gas sensitive field effect transistors has, so far, not been systematically studied, and only very basic studies with silicon based FETs can be found in literature. In this project, the virtual multisensor approach is applied to SiC-FETs and extended by gate bias cycled operation, GBCO, cf. Chapter 7, as well as improved signal processing, cf. Section 7.4. 


\section{Signal Processing}

Signal processing for chemical sensors is also known as chemometrics [155] which deals with the analysis of chemical data, extracting information from data by using mathematical and statistical methods [214]. In the era of electronic noses [158], signal processing for gas sensor arrays, mostly based on pattern recognition, has been pushed further [215], [216], [217], [218].

Multidimensional data from either a real sensor array or from a virtual multi-sensor usually contains a lot of redundancy and is most often also affected by noise and drift. Thus, the main demand on the evaluation algorithm is the reduction of dimension by projection of the data into a lower dimensional space. The established procedure of signal processing for virtual multi-sensors [219] can be divided into three main parts as shown in Fig. 5.1: 1) pre-processing or baseline correction, 2) the core of the signal processing, the pattern analysis or reduction of dimension, and 3) post-processing or classification. The first two parts constitute the training or training phase while the third part is the evaluation or classification of new, unknown data based on the training. Classification is also used in the training phase for validation of the discrimination.

The goal of the signal pre-processing is to prepare the data for the feature extraction and the following pattern analysis (for electronic noses see also [150]). Basically, the noise and drift effects need to be compensated for as much as possible. Algorithms and strategies are discussed in Section 5.1.

The most critical part of the signal processing is the feature extraction. Here, gas specific parameters need to be extracted which are the basis for pattern analysis and have a significant impact on its performance, cf. Section 5.2. By calculating features from the pre-processed data of a virtual multi-sensor, the dimension which is given by the number of sample points per cycle for a virtual multi-sensor will be reduced. For sensor arrays the dimension of the raw data set is given by the number of 
sensors. In that case, feature extraction usually enlarges the dimension since several features per sensor are extracted.
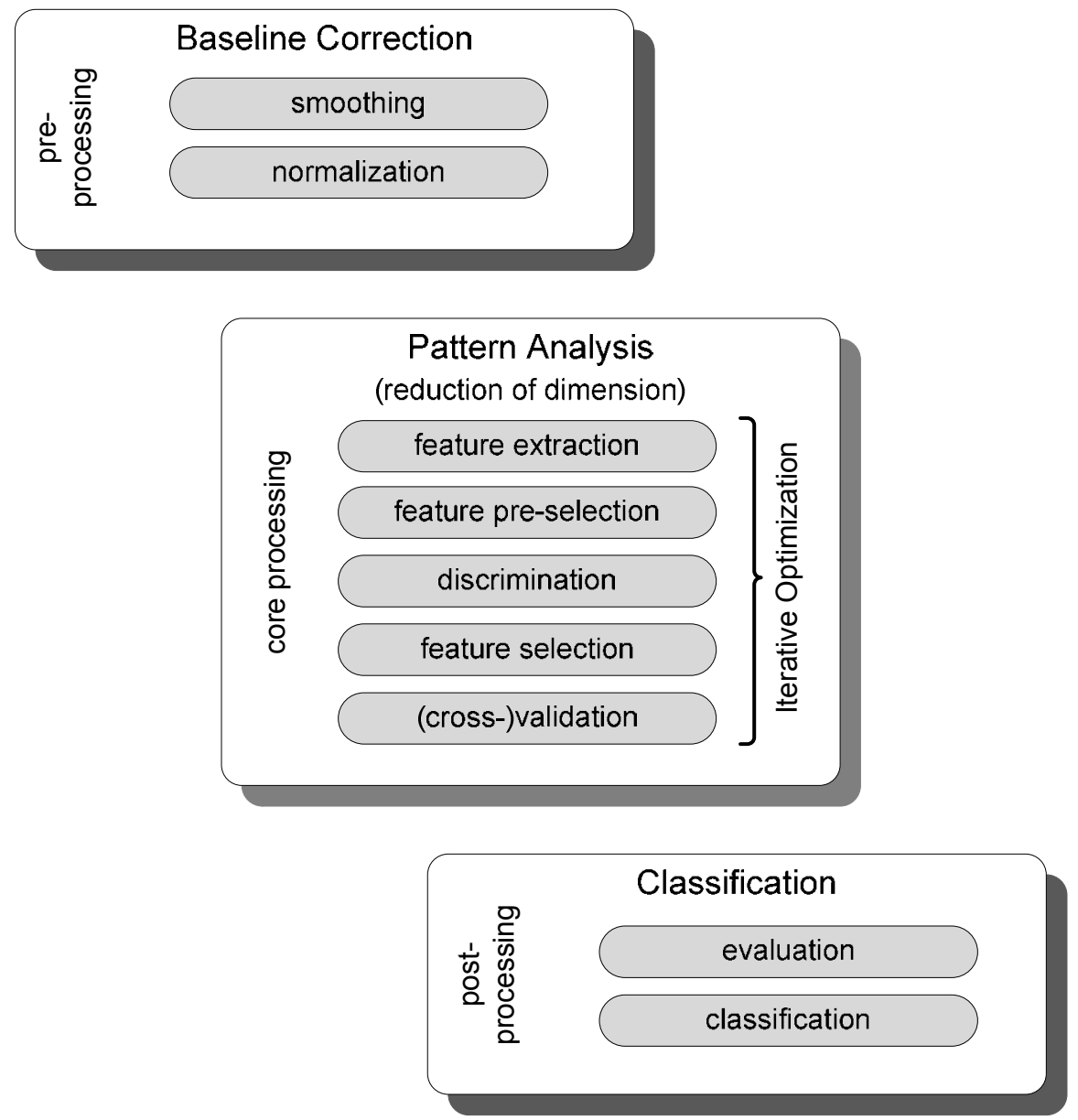

Fig. $5.1 \quad$ Signal processing flow chart.

Several pattern analysis algorithms have been proposed for electronic noses and (virtual) sensor arrays, cf. Section 5.3. Commonly used are Principal Component Analysis, PCA, and Linear Discriminant Analysis, LDA, which reduce the dimension by projecting the data into a lower dimensional space ${ }^{11,12}$. The stability of the discrimination can be checked by (cross-)validation, cf. Section 5.5. Principal

\footnotetext{
${ }^{11}$ In fact only LDA reduces the dimension. Usually the number of features is larger than the number of classes to be discriminated. Then, the dimension of the LDA projection is equal to the number of classes minus 1 . The dimension of a PCA projection is equal to the number of features but since only a few principal components are usually needed to achieve a good discrimination, PCA practically reduce the dimension, too.

${ }^{12}$ LDA is often seen as a classifier. However, in this context LDA refers to canonical discriminant analysis which only does projection. For classification purposes, a separate classifier is needed.
} 
Component Analysis is sometimes also applied as a feature extraction method. The obtained principal components are then used as input data, i.e., features for pattern recognition algorithms like LDA, cf. Section 5.2.1. However, in this work mainly LDA is used for processing of priorly extracted features describing the shape of the sensor response. The main advantage of LDA is the fact that LDA is a supervised algorithm while PCA is unsupervised ${ }^{13}$.

After training, the built model can be used to classify new, unknown data points. Depending on the chosen algorithm a separate classifier is needed, cf. Section 5.4.

\subsection{Baseline Correction}

Sensor responses from chemical sensors are often affected by drift and different kinds of noise, e.g., thermal noise which is sensor device related, or digital noise which is hardware related, as well as artefacts, e.g., outliers. Signal pre-processing is necessary to compensate for these unwanted effects. While outliers can be identified and removed from the data by simply setting a threshold limit, filters or smoothing algorithms are effective methods to reduce noise. Drift effects can be eliminated by a recalibration of the system from time to time but this is expensive and often not feasible. Thus, normalization of the raw data is done in order to eliminate or reduce drift. For smoothing and normalization, both local and global methods can be applied. Global methods operate usually on the entire data set, for instance scaling of the entire data set, or on large parts of the data set. In contrast to that, local algorithms operate only on smaller partitions, e.g., across the sensor array ${ }^{14}$ or on a single cycle for virtual multi-sensors.

For static sensor operation, this step is sometimes called baseline correction or baseline manipulation [150]. The baseline of a sensor operated in a cyclic mode is more complex. In fact, there is no single baseline as each point of a cycle plotted over the cycle number or time, i.e., quasi-static sensor response, can be seen as a sensor signal at a certain temperature and / or bias. In dynamic mode, the smallest reasonable unit is one cycle and thus, local methods are usually applied on a cycle-

\footnotetext{
${ }^{13}$ Supervised means that the group relationship of each data point is known beforehand and used for the discrimination. Unsupervised algorithms do not take the group relationship into account for the discrimination process. See Section 5.3.

${ }^{14}$ Local methods operate on the entire array, i.e., on all sensors, at a certain point in time.
} 
basis [220], for instance scaling of each cycle individually. Smoothing and normalization are both optional and often only normalization is needed. Also the order is not pre-defined; in some cases it might be more conceivable to normalize first and smooth the data afterwards.

\subsubsection{Smoothing}

The goal of smoothing is the removal of as much noise as possible without, at the same time, unduly degrading the underlying information. No matter which kind of smoothing is used, some of the information contained in the sensor signal is lost and depending on the chosen algorithms and parameters data can significantly be manipulated. For instance, the shape of the sensor response can be changed by smoothing algorithms, which is the basis for the feature extraction later on, cf. Section 5.2. Additionally, sharp steps in the signal which can occur in the signal of a gas sensor due to gas response might be flattened somewhat. It was reported that the running-in process of a gas is characteristic and important for the determination of the particular gas [169].

In general, smoothing algorithms act like filters: only a certain part of the incoming signal is able to pass the filter. Quite often the sensor response is affected by noise of high frequency so that a low-pass filter is conceivable.

There are several ways to smooth data which can be divided into two main groups. On one hand, algorithms which directly manipulate data like moving average. Here, a data point from a cycle is replaced by the average of the neighboring data points defined within a certain span [221]. On the other hand, there are algorithms that fit functions to the raw data. For instance a k-order polynomial can be fitted to the raw data. The higher the order $k$, the better is the approximation, but if the order is too high, the resulting curve tends to have overshoots and the result becomes worse. Thus, the order $k$ needs to be chosen carefully. Instead of a polynomial the sensor signal can also be fitted using exponential functions or smoothing splines [222], [155]. A welcome side effect of smoothing with fitting curves is that parameters of the new curve can be used as features for the pattern recognition [169]. Reimann used coefficients of Legendre polynomials [223] fitted to the sensor signal 
as features for the pattern recognition [8]. Although, this was not his original intention, this approximation is a kind of filtering or smoothing, of course.

Another well-known smoothing filter is the Savitzky-Golay Convolution filter, SG-filter, which was first reported in Analytical Chemistry in 1964 [224] with minor errors being corrected later on [225], [226]. The algorithm can be seen as a generalized moving average filter. The SG-filter is often used with spectroscopic or frequency data. The main advantage of the SG-filter is that it tends to preserve features, e.g., relative minima and maxima or higher moments of peaks in spectroscopic data. These features are usually lost or at least altered when using moving average filters.

\subsubsection{Normalization}

There are different ways to normalize data taking into account different sensor drifts, like additive drift which is a shift of the sensor's baseline, or multiplicative drift which affects the sensor response, i.e., sensitivity [171], or a combination of both. Hence, an appropriate normalization algorithm needs to be applied in order to effectively compensate for sensor drift. For virtual multi-sensors based on resistive type metal oxide gas sensors, a multiplicative normalization of the cycle mean where the cycle mean is set to one has been successfully applied to various applications [205]:

$$
y_{i, j}^{n o r m}=\frac{y_{i, j}}{\operatorname{mean}\left(y_{i}\right)}=\frac{y_{i, j}}{\frac{1}{p} \sum_{j=1}^{p} y_{j}}
$$

where $y_{i, j}$ and $y_{i, j}^{\text {norm }}$ are the raw and normalized data points $j$ within a cycle $i$ and $p$ the number of data points per cycle. Another possibility for multiplicative normalization is setting the last cycle value to one [227] or in general any other part of the cycle, i.e., dividing all values by $y_{j}$.

In contrast to that, additive normalization means to subtract a certain value of the cycle, for instance the cycle mean value:

$$
y_{i, j}^{n o r m}=y_{i, j}-\operatorname{mean}\left(y_{i}\right)=y_{i, j}-\frac{1}{p} \sum_{j=1}^{p} y_{j}=y_{i, j}-\bar{y}_{i}
$$


Additionally, normalization scales the cycle and thus, focuses the discrimination on the change in shape due to gas response rather than on absolute values. However, it should be noted that normalization also means loss of information; depending on the normalization certain parts of the cycle can be stressed whereas discriminatory information in other parts is suppressed. When setting the mean of each cycle to one, information regarding discrimination of different gases which is contained in the raw data close to the mean of the cycle, is discarded. For dynamically operated MOX sensors, the average resistance is typically removed which is in static operation usually the main feature. Nevertheless, this normalization lead to improved results regarding the quality of discrimination and the stability.

However, there are also other methods for normalization, like mapping each cycle into an interval, e.g., $[-1 \ldots 1]$ :

$$
y_{i, j}^{\text {norm }}=\frac{2 \cdot\left(y_{i, j}-\min \left(y_{i}\right)\right)}{\max \left(y_{i}\right)-\min \left(y_{i}\right)}-1
$$

Besides the above mentioned normalizations there is also the possibility to set the length, i.e., norm, of each cycle, Eq. (5.4), or the standard deviation, Eq. (5.5), of each cycle to one:

$$
\begin{gathered}
y_{i, j}^{\text {norm }}=\frac{y_{i, j}}{\operatorname{norm}\left(y_{i, j}\right)}=\frac{y_{i, j}}{\sqrt{\sum_{j=1}^{p} y_{j}^{2}}} \\
y_{i, j}^{\text {norm }}=\frac{y_{i, j}}{\operatorname{std}\left(y_{i, j}\right)}=\frac{y_{i, j}}{\sqrt{\frac{1}{p-1} \sum_{j=1}^{p}\left(y_{j}-\bar{y}_{i}\right)^{2}}}
\end{gathered}
$$

and additionally with a cycle mean of zero:

$$
y_{i, j}^{\text {stand }}=\frac{y_{i, j}-\operatorname{mean}\left(y_{i}\right)}{\operatorname{std}\left(y_{i}\right)}=\frac{y_{i, j}-\bar{y}_{i}}{\sqrt{\frac{1}{p-1} \sum_{j=1}^{p}\left(y_{j}-\bar{y}_{i}\right)^{2}}}
$$

This normalization where the standard deviation of each cycle is set to one and cycle mean to zero is sometimes called sensor auto-scaling [150], [219]. 
If and which kind of normalization gains the optimal performance generally depends on various factors: first, the optimal normalization depends on whichever gases should be discriminated or quantified. If different gases change the shape of the sensor response whereas different concentrations of particular gas only lead to a shift of the sensor response, normalization needs to be chosen carefully. Second, the normalization depends on the chosen cycle. Third, it depends on the used sensors. Different sensors respond differently and have different drift behavior. Thus, normalization needs to be adapted to this. Forth, depending on the next signal processing step, i.e., the feature extraction, different normalization can be adequate. If features describing the shape of the sensor response should be used, a normalization algorithm which highlights the change in the response should be applied. In general, it is very demanding to predict which kind of normalization will be the best with respect to the discrimination of gases and suppression of unwanted sensor response, e.g., influence of humidity, long-term stability, etc. 


\subsection{Feature Extraction and Pre-Selection}

Feature selection is the most important step in signal processing since here parameters need to be found which are gas-specific. In other words (after [228]), any selected feature

- must discriminate between at least two classes, e.g., gas or concentration

- must not be correlated with other selected features ${ }^{15}$

- should represent changes in the sensor response due to gas interaction

- should not represent drift or aging affects

- should be robust

Usually, many features are extracted; cf. Section 5.2.1, at first glance and the most significant ones are selected after discrimination. This is called top-down approach. A bottom-up approach is in general possible as well but more challenging to implement since it is rather difficult to determine a good feature beforehand. Therefore, a modified bottom-up approach can be used in which a strong emphasis is put on feature pre-selection, cf. Section 5.2.2, i.e., before discrimination, often based on descriptive statistics. In the present thesis, a top-down approach is favored consisting of a feature extraction and a feature selection step after discrimination based on multivariate statistics, cf. Section 7.4.

\subsubsection{Feature Extraction}

The main purpose of feature extraction is to find a number of informative parameters, i.e., features that preserve most of the information in the original data set but with a much lower dimension. This stage can be seen as mapping of the preprocessed data into a lower dimensional space spanned by these features. There is always a need to come to a compromise between the number of dimensions which highly correlates with the required computational effort and the loss of information. Feature extraction and selection often require multiple steps or iterations together with the pattern recognition algorithm later on in order to find the optimal set of

\footnotetext{
${ }^{15}$ This is an ideal assumption. Depending on the pattern recognition algorithm later on, correlating features are automatically excluded or can easily be identified.
} 
features, cf. Fig. 5.1. There are basically to ways for obtaining these features, after [171]: the empiric method, and the algorithmic method. The empiric method is based on the intuition and the experience of the user whereas the algorithmic method tries to find features systematically. In fact, there is no consensus on the best technique for feature extraction and several approaches have been suggested. Marco and Gutiérrez-Gálvez published a review on several feature extraction methods [218]: algorithmic based methods which, for instance, do direct projection of input data into a subspace, and curve fitting methods which usually have an empiric nature. As algorithmic based methods, typically linear methods like Principal Component Analysis, PCA [229], [230] (cf. Section 5.3.2) or Linear Discriminant Analysis, LDA, [231], [232] (cf. Section 5.3.3) are used as well as Fast Fourier Transform, FFT, [233] and Discrete Wavelet Transform, DWT [228], [234]. For instance, the obtained principal components, PC, are then used as features for classification algorithms or, in general other pattern recognition tools like LDA. Discriminant Analysis of principal components is a commonly used technique in face recognition [235], [236], [237]. The advantage of combing PCA and LDA is that all calculated principal components are orthogonal to each other, i.e., linear independent, which is a requirement for many algorithms, in particular for LDA, cf. Section 5.3. The method has also been applied to the field of chemical sensing, e.g., for spectroscopic data [238] and large redundant sensor arrays [239]. Besides calculating PCA first and using the obtained PCs as features for LDA, Wang et al. suggested Principal Discriminants Analysis, PDA, which is a real combination of PCA and LDA [240]. However, this is so far not a standard algorithm and there is only limited experience with it. Vergara et al. have introduced other transient features based on a series of Bessel functions and exponential moving average, EMA [241], or features from sensor waveforms in temperature-programmed sensing, TPS, from micro-sensor arrays, the so called energy vector, EV [242], [243].

After each change in operating temperature, the sensor response is affected by two things: temperature change which changes the conductivity of the semiconductor, and the gas effect which strongly influences the transient sensor response. Thus, as curve fitting methods, an exponential function Eq. (5.7) can be fitted to the sensor response. 
The obtained coefficients $\mathrm{A}, \mathrm{B}$, and $\mathrm{C}$ as well as the time constants $\tau_{1}$ and $\tau_{2}$ are used as features [244]:

$$
y=A \cdot e^{\frac{x}{\tau_{1}}}+B \cdot e^{\frac{x}{\tau_{2}}}+C
$$

Besides the physically motivated exponential function also different kinds of polynomials, e.g., Legendre Polynomials [245], and their coefficients can be used.

Another empiric and very promising approach is the use of shape-describing features. Here, mean values, secants, slopes, or differences which are calculated piecewise in each cycle are extracted as features [19]. In addition to these primary features, Engel [206] introduced delta values which are differences of, e.g., two mean values. These features are called secondary features. It should be noted that features need to be uncorrelated or linearly independent for use in pattern recognition afterwards. Due to that, it is not possible to use delta values and the corresponding mean values as features at the same time ${ }^{16}$.

The main advantage of shape-describing features is the simple calculation and implementation of the calculation in a micro-controller where computational power is often extremely limited. Further, it was reported that shape-describing features outperform features generated by FFT and DWT [220]. Shape-describing features have first been introduced to resistive type metal oxide sensors [19] but lately also for gas sensitive field effect transistors [246], [219].

\subsubsection{Feature Pre-Selection}

In feature pre-selection, features which obviously represent noise or sensor drift are usually excluded from the feature set. This pre-selection is independent of the learning algorithm to be used later on. A parametric way for pre-selection is based on descriptive statistics. The most common way of graphically expressing the results of descriptive statistics is the box-and-whisker plot or short box plot [155]. In addition, the correlation of each feature with the gas exposure can be calculated and only features with a high correlation are selected [247]. Similar to this approach, the

\footnotetext{
${ }^{16}$ This is especially the case for Linear Discriminant Analysis.
} 
correlation of features to any other feature can be calculated. Since the features should be linearly independent, they should be uncorrelated.

A rather simple way to identify not significant features is plotting the feature value over time which easily reveals noise features, see Section 7.4.1. However, this approach is only feasible for a small number of features. Besides this manual inspection, feature pre-selection can partly be automated. In particular, the analysis of correlation is easy to implement in a selection algorithm but validation of the selected features by the user is still recommended. In general, there is a tendency to automate all steps in the signal processing but this leads to a black-box-system which results are often barely comprehensible and mostly hard to interpret. Thus, for developing a new methodology manual inspection and selection of features is preferable to an automated process. 


\subsection{Multivariate Statistics}

Several different pattern recognition techniques for evaluation of sensor data have been suggested. Fig. 5.2 gives an overview of the most common techniques. For a more detailed description of the different algorithms readers are referred to [215], [155], [230] or other text books. In general, these algorithms can be divided into statistical and biologically inspired (non-parametric) techniques as well as in supervised and unsupervised methods. Supervised means, that the correct classification is known for each object prior to discrimination. Non-parametric algorithms are for instance Artificial Neural Networks, ANN, including SelfOrganizing Maps, SOM, Multi-Layer Perceptron, MLP, Probabilistic Neural Networks, PNN, Radial Basis Functions, RBF, and Learning Vector Quantification, LVQ. Artificial Neural Networks are self-learning algorithms which are inspired by biological neurons like nerve cells in the brain.

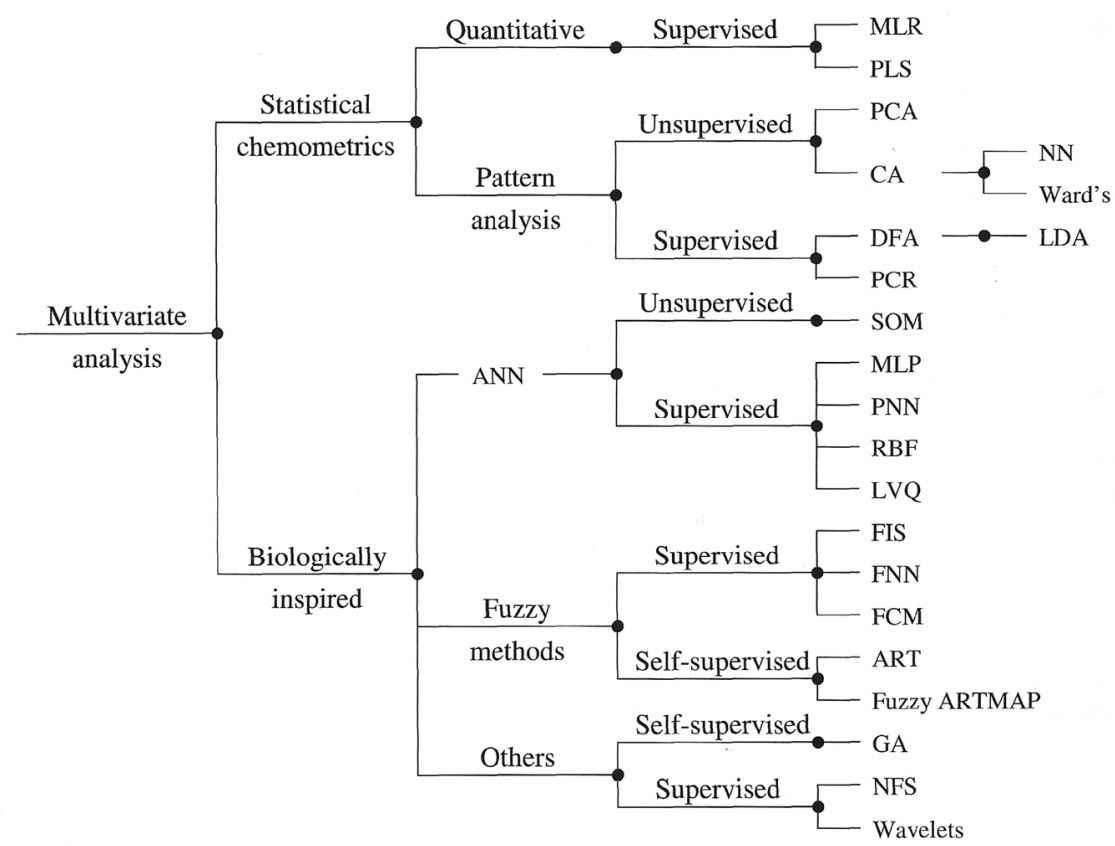

Fig. 5.2 Overview of multivariate pattern analysis techniques. Reproduced from [248] with kind permission from Wiley-VCH. 
The connection between two neurons is adapted which is called the learning process, i.e., the weight with which the output of the neuron is multiplied and given to the next neuron. The most well-known implementation is the multi-layer (often threelayer) feed-forward back-propagation trained network and has successfully been applied to sensor data [206], [218]. Besides ANN also Fuzzy logic like Fuzzy Inference Systems, FIS, Fuzzy Neural Networks, FNN, Fuzzy c-means, FCM, and Adaptive Resonance Theory, ART, are used. In contrast to probability theory, fuzzy theory is a possibilistic approach which means that for each input variable a membership function is given defining the grade of membership. The theory of Fuzzy sets was introduced by Zadeh in 1965 [249] and has been applied to several fields mostly in linguistics but also for electronic noses. In addition to ANN and fuzzy logic, some other algorithms have been proposed, like Generic Algorithms, GA, Neuro-Fuzzy Systems, NFS, and wavelets.

Another class of pattern recognition techniques based on statistics and their use for evaluating chemical sensor data is called chemometrics [214]. It can be distinguished between quantitative algorithms which are used to determine a concentration of a substance, e.g., quantification of a gas, and pattern analysis (qualitative) which generally deals with discrimination of different groups, i.e., the separation of different gases. For quantification usually regression is used, like Multiple Linear Regression, MLR, or Partial Least Squares Regression, PLSR, whereas pattern analysis is often done by Principal Component Analysis, PCA, Cluster Analysis, CA, Discriminant Function Analysis, DFA, or Principal Component Regression, PCR. Typically, these algorithms do not classify and therefore require a separate classifier afterwards, cf. Section 5.4. LDA and PCA as well as PLSR are discussed more deeply in the next sections since they are the most well-known parametric algorithms and used in this thesis.

\subsubsection{Basic Statistics}

In this sub-section, a few mathematical definitions will be presented which are important for the understanding of multivariate data evaluation, i.e., PCA and LDA later on. The descriptions are based on [155], [250]. 
In multivariable statistics, objects, i.e., measured variables like cycles, are characterized by a feature vector $\boldsymbol{x}$ containing $N$ extracted, ideally independent features (variables). A measurement set, i.e., the entirety of measured variables, of $M$ objects, e.g., cycles, and $N$ features is given as a matrix $\boldsymbol{X}$ in which each row represents a cycle. The features are stored in different columns.

$$
\boldsymbol{X}=\left(\begin{array}{cccc}
x_{1,1} & x_{1,2} & \ldots & x_{1, N} \\
x_{2,1} & x_{2,2} & & \\
\vdots & & \ddots & \\
x_{M, 1} & & & x_{M, N}
\end{array}\right)
$$

In order to transform the original data into a new space as it is done in Principal Component Analysis, the relationships among the data are important. This is reflected by the symmetric variance-covariance matrix cov with the variances $s_{j}^{2}$ (square of standard deviation $s$ ) on the main diagonal, i.e., $\operatorname{cov}\left(x_{j, j}\right)=s_{j}^{2}$, and their covariances on the minor diagonals. A covariance of zero means that the variables are entirely independent.

$$
s_{j}^{2}=\operatorname{var}\left(x_{j}\right)=\frac{1}{M-1} \cdot \sum_{i=1}^{M}\left(x_{i, j}-\bar{x}_{j}\right)^{2}
$$

with $M$ the number of cycles contained in the data set and $\bar{x}_{j}$ the mean of feature $j$ (column $j$ of the matrix $X$ ), $j=1 \ldots N$.

$$
\operatorname{cov}\left(x_{j, k}\right)=\frac{1}{M-1} \cdot \sum_{i=1}^{M}\left(x_{i, j}-\overline{x_{j}}\right) \cdot\left(x_{i, k}-\overline{x_{k}}\right)
$$

with $j, k=1 \ldots N$ and $j \neq k$.

$$
\operatorname{cov}\left(x_{j, k}\right)=\frac{{\widetilde{x_{J}}}^{\boldsymbol{T}} \cdot \widetilde{\boldsymbol{x}_{\boldsymbol{k}}}}{M-1}
$$

with $\widetilde{x_{j}}$ and $\widetilde{x_{k}}$ the centered feature vectors.

If the standardized feature vectors were used in Eq. (5.11) instead of the centered vectors, the correlation matrix would be derived.

For supervised learning algorithms like Linear Discriminant Analysis, the group relationship of each object (cycle) is known. The data set contains $G$ different groups 
or classes, and $I_{g}$ is the number of objects in class $g$. Besides the variance-covariance matrix cov, which characterizes the relationships among the objects, the withingroups sum of squares and cross-products matrix $\boldsymbol{W}$ describes the relation of objects in their corresponding group. In addition to $\boldsymbol{W}$, total sum of squares and crossproducts matrix $\boldsymbol{T}$ defines basically the differences between the groups. By dividing $\boldsymbol{W}$ and $\boldsymbol{T}$ by $M-G$ the within-group and total covariance matrices are derived.

$$
w_{j, \mathrm{k}}=\sum_{g=1}^{G} \sum_{i=1}^{I_{g}}\left(x_{j g i}-\overline{x_{\jmath g}}\right) \cdot\left(x_{k g i}-\overline{x_{k g}}\right)
$$

with $x_{j g i}$ the value of feature $j$ for cycle $i$ in group $g$ and $\overline{x_{j g}}$ the mean value of feature $j$ in group $g$.

$$
t_{j, k}=\sum_{g=1}^{G} \sum_{i=1}^{I_{g}}\left(x_{j g i}-\overline{x_{J}}\right) \cdot\left(x_{k g i}-\overline{x_{k}}\right)
$$

with $\bar{x}_{j}$ the overall mean value of feature $j$.

Further, the between-groups sum of squares and cross-products matrix $\boldsymbol{B}$ can be introduced and is usually given as the difference between $\boldsymbol{T}$ and $\boldsymbol{W}$.

$$
\begin{gathered}
\boldsymbol{B}=\boldsymbol{T}-\boldsymbol{W} \\
b_{j, \mathrm{k}}=\sum_{g=1}^{G} I_{g} \cdot\left(\overline{x_{\jmath g}}-\overline{x_{\jmath}}\right) \cdot\left(\overline{x_{k g}}-\overline{x_{k}}\right)
\end{gathered}
$$

\subsubsection{Principal Component Analysis}

The following derivations are based on text books by Dunteman [229], Backhaus [250], and Duda [230], additional information can be found in [155], [251].

Principal Component Analysis, PCA, is one of the most well-known and used algorithms for evaluating chemical sensor data. It is an unsupervised learning method that performs a coordinate transformation of the feature matrix $\boldsymbol{X}$ into a new space 
spanned by orthogonal principal components, PC, i.e., directions of maximum variance. Here, the first PC represents the main direction of the variance in the data set. The transformation can be derived by orthogonal decomposition of the feature matrix $\boldsymbol{X}$.

$$
X=U \cdot S \cdot V^{T}
$$

The matrix $\boldsymbol{U}$ contains the eigenvectors of the matrix $\boldsymbol{X} \cdot \boldsymbol{X}^{\boldsymbol{T}}$ and the matrix $\boldsymbol{V}$ the eigenvectors of $\boldsymbol{X}^{\boldsymbol{T}} \cdot \boldsymbol{X}$ which corresponds to the covariance matrix or, in case of a standardized feature matrix corresponds to the correlation matrix. The matrix $S$ is a diagonal matrix containing the square roots of the eigenvalues $\lambda_{i}$. The eigenvectors ordered in descending order of their corresponding eigenvalue are the principal components.

The new projection $\boldsymbol{t}$ of the input variables, i.e., the feature matrix $\boldsymbol{X}$, which is known as the scores, is obtained by simple multiplication of $\boldsymbol{V}$ which elements are called loadings with the features $\boldsymbol{X}$.

$$
\boldsymbol{t}=\boldsymbol{V} \cdot \boldsymbol{X}
$$

The dimension of $\boldsymbol{V}$ is $N \times N$, in other words the number of possible PCs is defined by the number of features, and in the most general case there is no reduction of dimension by transforming the data. However, the first two or three PCs often represent more than $90 \%$ of the variance. The other principal components, i.e., $\mathrm{PC}_{4}$ to $\mathrm{PC}_{\mathrm{N}}$, can usually be neglected without a significant loss of quality. How many functions are significant can be determined by the Scree-plot, cf. Fig. 5.3.

As can be seen, by using the first two PCs more than $90 \%$ of all the information is represented. The scores of the first up to three PCs can be plotted in the score or scatter plot. Different groups are colored differently but since PCA is unsupervised this information is not used for calculation of the transformation matrix. Not only the scores, the separation of the groups, is of interest but also which features contribute most to the result. 

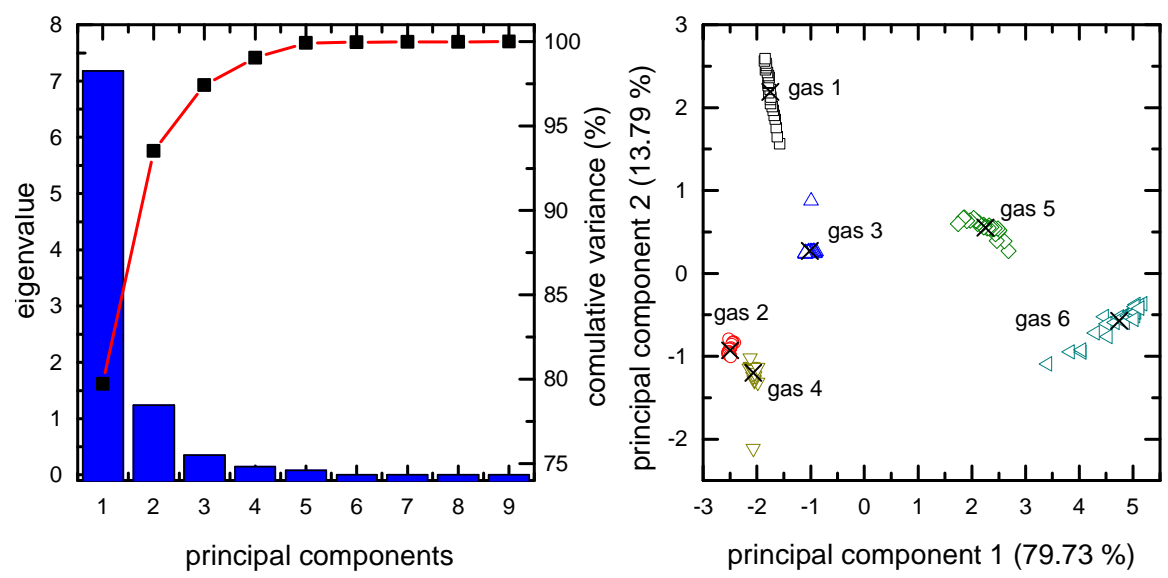

Fig. 5.3 (a) Scree-plot showing the eigenvalues (blue bars) and cumulative variance (red line) of a PCA. (b) Score plot of the first two principal components discriminating six gases.

The loadings plot, cf. Fig. 5.4a, visualizes the loadings, the coefficients for each feature. The higher the coefficient is, the more important the feature is. Hence, features which are located further away from the origin in the loadings plot are more important, whereas features close to the origin are insignificant. However, this is only true if the features are of the same magnitude. In order to overcome this issue, the features need to be standardized first. The loadings and score plot can be combined in the bi-plot, cf. Fig. 5.4b, showing loadings and scores at the same time.
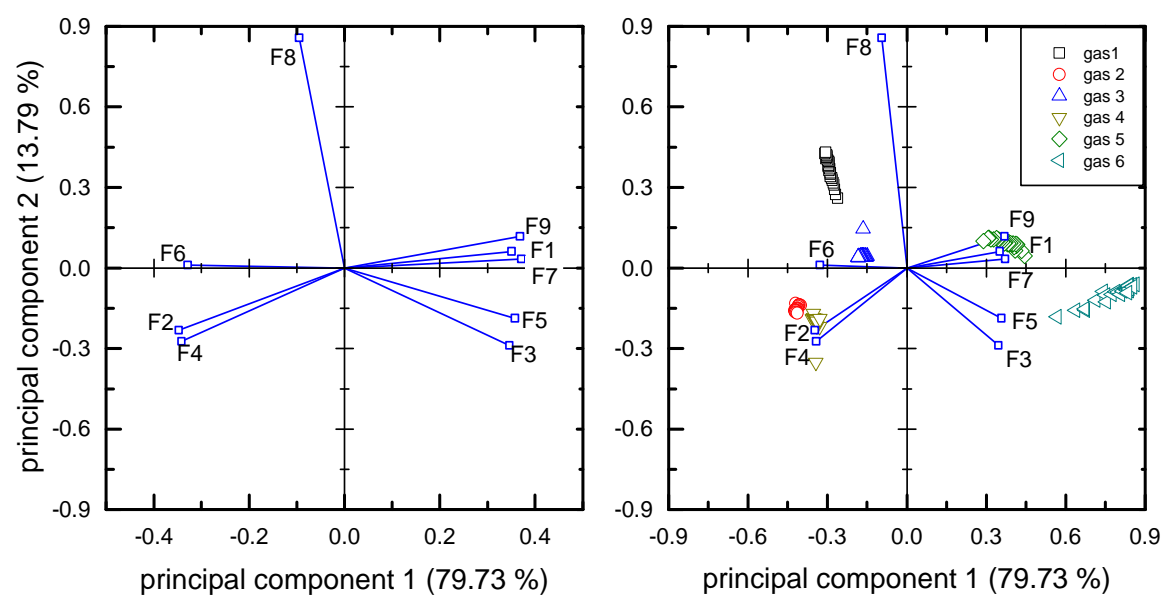

Fig. $5.4 \quad$ (a) Loadings plot of the PCA showing the impact of each feature F. (b) Biplot showing the loadings and the scores in one diagram. 
This plot is commonly used to study the influence of each feature and for selecting the most significant ones. As shown in Fig. 5.4b, features F8 mainly contributes to the separation of gas 1 whereas features F1, F7, and F9 separate gas 6. How features can be selected by using the loadings or bi-plot is discussed in detail in Section 7.4.1. In general, PCA has several benefits, adapted from [252]:

- Easy to calculate for fast inspection of the data

- Data representation is unbiased

- Identification of variable structure (similarities, influence of features, etc.)

- Results can be interpreted graphically

- Reduction of dimension (only a few PCs carry most of the information)

However, there are also some drawbacks of PCA:

- Unsupervised methods, class membership is not taken into account

- PCA is sensitive to noise and outliers

- Separation only possible if discriminative information is reflected in variance

- PCA requires careful weighting, e.g., standardization, of input variables

\subsubsection{Linear Discriminant Analysis}

The following derivations are based on text books by Klecka [232] and Backhaus [250], additional information can be found in [155], [230] and a collection of all important mathematical formulas is given in [253].

Linear Discriminant Analysis, LDA, transforms the input data $\boldsymbol{X}$ into a new space similar to PCA. However, LDA is a supervised learning method which means that the class relationship, the coding of each variable is known a priori and taken into account during transformation. Thus, not the direction of largest variance in the data set is sought but rather a linear combination that separates the different classes optimally. LDA was first developed by Fisher in 1936 [231] for discrimination and classification purposes of different kinds of irises, i.e., iris setosa, iris veriscolor, and iris virginica. After that, several computation methods have been developed to do discriminant analysis [254]. The term (Linear) Discriminant Analysis is rather broad and used inconsistently for several algorithms, unfortunately. In this work, Linear 
Discriminant Analysis refers to Canonical ${ }^{17}$ Discriminant Analysis to study the nature of group differences. It should be noted that with Canonical Discriminant Analysis only a projection of the data is done rather than a classification. A canonical discriminant function $Y$ is a linear combination of the discriminating variables $\boldsymbol{x}$ and the coefficients, i.e., loadings, $v$ which needs to be derived:

$$
Y=v_{0}+v_{1} \cdot x_{1}+v_{2} \cdot x_{2}+\cdots+v_{N} \cdot x_{N}
$$

The family of discriminant functions spans the discriminant space. In order to allow classification in this space, a separate classifier is needed, cf. Section 5.4.

The general idea of LDA is to maximize the distance between the groups while minimizing the inner group scatter. In other words, the discrimination criterion $\boldsymbol{\Gamma}$ is maximized.

$$
\boldsymbol{\Gamma}=\frac{\text { scatter } \text { between } \text { groups }}{\text { scatter } \text { within } \text { the } \text { groups }}=\text { max }
$$

The scatter between groups can be expressed by the squared distances of the groups centroids in the transformed space from the overall centroid, sum of squares between groups $S S_{B}$. The sum of squares within the groups $S S_{W}$ describes the scatter within each group. The relationship within and between the groups in the input space is described by the matrices $\boldsymbol{W}$ and $\boldsymbol{B}$ as introduced in Section 5.3.1.

$$
\boldsymbol{\Gamma}=\frac{S S_{B}}{S S_{W}}=\frac{\boldsymbol{u}^{\boldsymbol{T}} \cdot \boldsymbol{B} \cdot \boldsymbol{u}}{\boldsymbol{u}^{\boldsymbol{T}} \cdot \boldsymbol{W} \cdot \boldsymbol{u}}=\max
$$

Equation (5.20) leads to an eigenvalue problem

$$
\boldsymbol{B} \cdot \boldsymbol{u}=\lambda \cdot \boldsymbol{W} \cdot \boldsymbol{u}
$$

The eigenvectors $\boldsymbol{u}$ are sorted in descending order by their eigenvalues and are normalized afterwards ${ }^{18}$. The values of the corresponding (normalized) eigenvectors $v_{1} \ldots v_{n}$ are the unstandardized coefficients, i.e., loadings of the discriminant

\footnotetext{
${ }^{17}$ Canonical means linear combination of variables [180].

${ }^{18}$ Normalization $v_{i}=u_{i} \cdot \sqrt{M-G}$ with $M$ the number of variables (cycles) in the data set and $G$ the number of groups, is done to give the discriminant function $Y$ more desirable properties. The discriminant scores over all cycles will have zero mean and a within-groups standard deviation of one [165].
} 
function $Y$. The constant term $v_{0}$ in Eq. (5.18) does not contribute to the discrimination but scales the scores in a way that the overall centroid of all groups is located in the origin of the transformed system. In general, the number of positive eigenvalues $K$ cannot be greater than the number of groups $G$ minus one or the number of features $\mathrm{N}$, i.e., $K=\min \{G-1 ; N\}$. Thus, LDA leads to a real reduction of dimension. How many discriminant functions, DFs, are needed can be determined with help of the Scree-Plot analogous to PCA. Normally, not more than three DFs are needed to represent more than $90 \%$ of the discriminatory information. How much each function contributes to the discrimination is explained by the relative percentage:

$$
\text { rel.percentage } e_{k}=\frac{\lambda_{k}}{\lambda_{1}+\lambda_{2}+\cdots+\lambda_{K}}
$$

Besides the relative percentage, there are other criteria describing the importance of each discriminant function, e.g., canonical correlation, Wilks' Lambda and the thereof resulting significance as well as the residual Wilks' Lambda. Usually, these values are less important for interpreting gas sensor data and are not discussed further here. Interested readers are referred to [232], [250].

The quality of the discrimination can be measured by basically two techniques [255]: First, information content with parameters like the discrimination criterion $\boldsymbol{\Gamma}$, Eq. (5.20), itself can be used. However, it is not scaled and an interpretation of the absolute value is difficult.

Additionally, cluster separability $J$ can be calculated which describes the ratio between between-scatter and total-scatter [256].

$$
J=\frac{\operatorname{trace}(\boldsymbol{B})}{\operatorname{trace}(\boldsymbol{B})+\operatorname{trace}(\boldsymbol{W})}
$$

In literature [232], [250], another parameter, the multivariate Wilks' Lambda is suggested to test if the discrimination is significant. It is an inverse measure and scaled to $[0 \ldots 1]$.

$$
\Lambda_{m u l t i}=\prod_{k=1}^{K} \frac{1}{1+\lambda_{k}}
$$


However, all these criteria are sometimes hard to interpret and all of them depend on the number of degrees of freedom, i.e., number of features and groups, so that comparison between different LDAs is often not possible. The second technique for measuring the performance of discrimination is predictive accuracy, like crossvalidation, cf. Section 5.5 .

There are also some statistics for each variable (here: feature) which can be used for feature selection. Here, only the basic formulas are given and feature selection itself is treated more deeply in Section 7.4.1. The LDA coefficients $v_{k, i}$ which are the prefactors of the features $x_{i}$ in the discriminant function $D F_{k}$, Eq. (5.18), are also a measure of how much the corresponding feature contributes to the discrimination along $D F_{k}$. However, the value of the unstandardized coefficient is not meaningful at all since it depends highly on the value of the feature itself. Slopes are for instance one magnitude smaller than mean values; hence coefficients from slopes are much larger than the ones from mean values. In order to compare the coefficients and thus, to select the most important features, the standardized coefficients need to be calculated.

$$
v_{i}^{*}=v_{i} \cdot \sqrt{\frac{w_{i i}}{M-G}}
$$

with $M$ the number of all objects (here: cycles) and $G$ the number of groups. The most intuitive parameter for selecting features is the average coefficient $\bar{v}_{l}$ which is the corresponding numerical value to the loading plot. It gives the average value of each coefficient over all possible DFs with respect to the importance of each DF.

$$
\bar{v}_{l}=\sum_{k=1}^{K}\left|v_{k, i}^{*}\right| \cdot \frac{\lambda_{k}}{\lambda_{1}+\lambda_{2}+\cdots+\lambda_{K}}
$$

More statistical parameters for each feature are the univariate Wilks' Lambda $\Lambda_{\text {uni }}$ as well as the univariate $F$-value, $F_{\text {uni }}$ :

$$
\begin{gathered}
\Lambda_{u n i_{i}}=\frac{w_{i i}}{t_{i i}} \\
\mathrm{~F}_{\text {uni }_{i}}=\frac{\left(t_{i i}-w_{i i}\right) \cdot(N-G)}{w_{i i} \cdot(G-1)}
\end{gathered}
$$


In general, LDA has several benefits, adapted from [252]:

- Optimized projection for further classification (supervised method)

- Identification of significant variables

- Results can be interpreted graphically

- Reduction of dimension

However, there are also some drawbacks of LDA:

- Risk of over-fitting (supervised learning)

- Individual weighting of variables not possible

- Different variances of classes are not considered

\subsubsection{Stepwise Linear Discriminant Analysis}

Stepwise $L D A$ is an automated method for selecting a nearly optimal feature subset. Several different algorithms, one way (forward or backward) or two-way selection, with different selection criteria, e.g., Wilks'Lambda, Rao's V, or Mahalanobis distance, have been suggested [232]. In the toolbox developed and used at the Lab for Measurement Technology, cf. Section 6.6, a combination of forward- and backward selection based on the partial F-ratio is used [250]. In a first step, the variable which provides the greatest univariate discrimination is selected based on the largest (univariate) $\mathrm{F}$-value ${ }^{19}$. Then, only features which have an F-to-enter value greater than a pre-defined, critical value (SPSS default value 3.84 [257]) are included. In the backward selection step, features are excluded if their F-to-remove value is below a threshold (SPSS default value 2.71 [257]).

In general, stepwise LDA does not necessarily lead to the best discrimination but most often leads to a good selection of features. However, it is a black-box approach and the obtained results can hardly be interpreted.

\subsubsection{Linear Regression}

In contrast to the earlier discussed algorithms like LDA and PCA, which give qualitative information, regression algorithms perform classification in respect to the concentration, i.e., provide quantitative information. The general idea of regression is

\footnotetext{
${ }^{19}$ Or the smallest (univariate) Wilks` Lambda values which leads to the same result.
} 
to predict a target value $\boldsymbol{Y}$ from the input $\boldsymbol{X}$. The simplest algorithm is Multiple Linear Regression, MLR, which finds a linear relationship between the response variable $\boldsymbol{Y}$ and several predictor variables $\boldsymbol{X}$ using the Ordinary Least Squares approach, OLS:

$$
\boldsymbol{Y}=\boldsymbol{X} \cdot \boldsymbol{C}+\boldsymbol{R}
$$

where $\boldsymbol{C}$ is a matrix with regression parameters and $\boldsymbol{R}$ a matrix containing the residuals. The parameter matrix $\boldsymbol{C}$ is determined by minimizing the sum of squared residuals. The solution of this problem is:

$$
C=\left(X^{T} \cdot X\right)^{-1} \cdot X^{T} \cdot Y
$$

However, in practice $\left(\boldsymbol{X}^{\boldsymbol{T}} \cdot \boldsymbol{X}\right)$ is often ill conditioned due to the fact that the variables $\boldsymbol{X}$, i.e., features, are to some extent correlated which causes instabilities of numerical algorithms when calculating the inverse $\left(\boldsymbol{X}^{T} \cdot \boldsymbol{X}\right)^{-\mathbf{1}}$.

Instead of applying the least squares approach directly on the features, a new set of variables, i.e., principal components, can be calculated first, which are then used as input for linear regression. In Principal Component Regression, PCR, the predictor variable $\boldsymbol{X}$ in Eq. (5.29) is replaced by its orthogonal decomposition, similar to Eq. (5.16).

$$
\boldsymbol{C}=\boldsymbol{X}^{*} \cdot \boldsymbol{Y} \text { with } \boldsymbol{X}^{*}=\boldsymbol{V} \cdot \boldsymbol{d i a g}\left(1 / \sqrt{\lambda_{i}}\right) \cdot \boldsymbol{U}^{T}
$$

where the matrix $\boldsymbol{V}$ contains the eigenvectors of $\boldsymbol{X}$ and $\lambda_{i}$ are the corresponding eigenvalues. The matrix $\boldsymbol{U}$ contains the eigenvectors of $\boldsymbol{X} \cdot \boldsymbol{X}^{\boldsymbol{T}}$. Basically, PCR performs a PCA first, and then does the regression using the principal components.

Another possibility of decomposing $\boldsymbol{X}$ is using the NIPALS (Nonlinear Iterative Partial Least Squares) algorithm [258], [259], [260] or SIMPLS (Straightforward Implementation of a Statistically Inspired Modification of the PLS method) [261] which is used among others by MATLAB. Typically SIMPLS is faster than NIPALS [262].

An extension of PCR is Partial Least Squares Regression, PLSR, which uses the information in $\boldsymbol{Y}$ to calculate PC optimized for a linear representation of data. Thus, 
PLSR can be seen as supervised PCR. In PLSR principal components are calculated for both input variables $\boldsymbol{X}$ and response $\boldsymbol{Y}$, after [252]:

$$
X=\widetilde{T} \cdot P^{T}, \quad Y=\widetilde{T} \cdot Q^{T}, \quad \widetilde{T}=X \cdot \widetilde{W}
$$

where $\boldsymbol{P}$ is the X-loadings matrix, $\boldsymbol{Q}$ the Y-loadings matrix, and $\widetilde{\boldsymbol{T}}$ the scores matrix. The weight matrix $\widetilde{\boldsymbol{W}}$ is calculated in a way that the covariance between $\boldsymbol{X}$ and $\boldsymbol{Y}$ is maximized.

$$
C=\widetilde{W} \cdot\left(P^{T} \cdot \widetilde{W}\right)^{-1} \cdot Q^{T}
$$

The advantage of PLSR over MLR is that it still leads to good results even if some of the predictors, i.e., features, show multi-collinearity since the resulting PLSR components are orthogonal. Due to the fact that also the response $\boldsymbol{Y}$ is taken into account for optimization, PLSR typically leads to slightly better results than PCR.

\subsubsection{Partial Least Squares - Discriminant Analysis}

Partial Least Squares - Discriminant Analysis, PLS-DA, is a discrimination and classification algorithm based on PLSR [263]. Classification is done based on a regression model, i.e., the PLSR model, but with a categorical instead of a continuous response. For more than two classes, a response matrix $\boldsymbol{Y}$ is used where each column represents one class. Class membership is indicated by 1 and nonmembership by 0 . Multivariate PLSR leads to a set of regression coefficients for each class. Each set will project the data in a way that one class will be located near 1 , while all other classes will be located near 0 , i.e., discriminates one class against all others. Hence, a discrimination threshold can be set around 0.5, above which unknown data will be classified as belonging to a specific class. However, this threshold can be set independently which can be used to adjust the classifier.

The advantage of PLS-DA over LDA is that PLS-DA works also for correlated features. Additionally, (canonical) LDA needs a separate classifier where as PLS-DA can directly classify data by the set threshold value. However, in theory, i.e., when all requirements for LDA are fulfilled, LDA will outperform PLS-DA [263]. 


\subsection{Classification}

The aim of classification is to assign new data points which have not been considered for discrimination before, to classes contained in the training. There are pattern recognition algorithms which themselves also classify new objects, e.g., Artificial Neural Networks, ANN, or Support Vector Machines, SVM, however in this section only classifiers which are commonly used after discrimination by either PCA or LDA are briefly discussed.

For an overview of other classifiers used for chemical sensors see [264], [155], [216], [218].

\subsubsection{K-Nearest Neighbor Classifier}

The $k$-Nearest Neighbor Classifier, kNN, is one of the simplest algorithms. The basic idea is to measure the distance to the $k$-neighbors nearest of an unknown ${ }^{20}$ object. The unknown object is classified into the group which is mostly represented within the $k$ neighbors. Usually, the distance is measured by the Euclidean distance.

$$
D_{\text {Euclidean }}=\sqrt{\sum_{k}\left(y_{k}^{\text {unknown }}-y_{k}\right)^{2}}
$$

The kNN classifier is more robust against outliers in the training set compared to a simple distance classifier. Besides the Euclidean distance basically any means of distance measure, e.g., Minkowski, Cheybychev, or Mahalanobis distance (see next section), is conceivable. It can be useful to weigh the contribution of neighbors by their distance. Neighbors that are nearer to the point to be classified are rated higher, i.e., contribute more. The value $k$ is usually in the range of $2 \ldots 10$; often $k$ is equal 3 or 5 .

\footnotetext{
${ }^{20}$ An unknown object is an object that was projected into a PCA or LDA score plot and that has not been used for deriving this score plot, the training.
} 


\subsubsection{Mahalanobis Distance Classifier}

A generalization of the Euclidean distance is the Mahalanobis distance $D_{\text {Mahal }}$ which calculates the distance of a (here: feature) vector $\boldsymbol{x}$ to the centroid of a class $\overline{x_{g}}$ :

$$
D_{\text {Mahal }}=\sqrt{\left(x-\overline{x_{g}}\right)^{T} \cdot \operatorname{Cov}_{g}^{-1} \cdot\left(x-\overline{x_{g}}\right)}
$$

where $\operatorname{Cov}_{\boldsymbol{g}}$ is the within-group covariance matrix of group $g$. The advantage of the Mahalanobis distance is that different scaling of the input variables is compensated for. Graphically spoken, the Mahalanobis distance weights the distance of an object to a group by the standard deviation of the group. Classification based on the Mahalanobis distance can also be performed on the canonical discriminant functions. For this, the $x$ in Eq. (5.35) needs to be replaced by the discriminant scores $y$.

According to [250] classification based on Euclidean distances in the discriminant space leads to the same results as classification based on Mahalanobis distance in the feature space. This is because the covariance matrix of the discriminant values becomes the identity matrix if equal scatter of the groups can be assumed. A more general definition of the Mahalanobis distance, which should be used in particular if the groups do not have equal covariance matrices, is given as, after [250]:

$$
D_{\text {Mahal }_{i g}}=\sqrt{\left(\boldsymbol{y}_{\boldsymbol{i}}-\overline{\boldsymbol{y}_{\boldsymbol{g}}}\right)^{T} \cdot \boldsymbol{C o v}_{\boldsymbol{g}}^{\mathbf{1}} \cdot\left(\boldsymbol{y}_{\boldsymbol{i}}-\overline{\boldsymbol{y}_{\boldsymbol{g}}}\right)}+\ln \left(\operatorname{det}\left(\operatorname{Cov}_{g}\right)\right)
$$

\subsubsection{Territorial Plot}

In general, different classifiers lead to different classification results and to different correct classification rates. In order to compare classifiers, territorial plots, also known as territorial maps, can be used. A territorial plot illustrates how unknown data points projected into the scatter plot would be classified. In Fig. 5.5a a LDA scatter plot showing the discrimination of three classes is given. In order to draw a territorial plot, a grid in the scatter plot is defined and each node of the grid represents an unknown data point to be classified. The corresponding territorial plot 
for a Mahalanobis distance classifier is shown in Fig. 5.5b, and for a k-Nearest Neighbor classifier with $k$ equals 5 is given in Fig. $5.5 \mathrm{c}$.
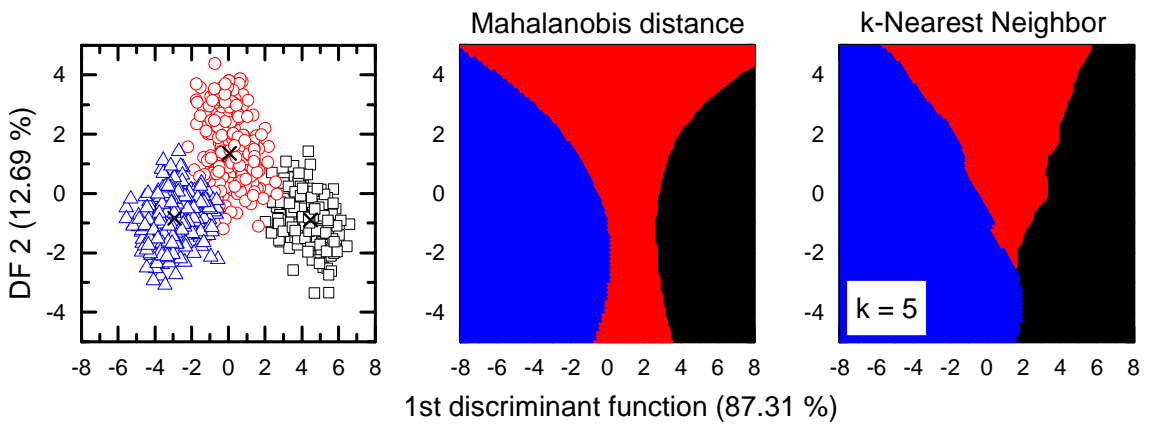

Fig. 5.5 (a) LDA scatter plot showing the discrimination of three classes, (b) territorial plot of a Mahalanobis distance classifier, and (c) territorial plot of a k-Nearest Neighbor classifier with $\mathrm{k}=5$.

It can be observed that both classifiers lead to different results. Whereas the borderlines for a Mahalanobis classifier are usually smooth, they can be quite rough for a $\mathrm{kNN}$-classifier depending on the parameter $k$. In general, the $\mathrm{kNN}$-classifier always leads to coherent areas in the territorial plot while in case of a Mahalanobis classifier it can happen that isolated islands of classes appear being apart from their main area. This can happen because the Mahalanobis distance takes the direction of the scatter of the class into account which is problematic if a class is not compact but rather consists of two or more clusters. Thus, a classifier needs to be chosen carefully in order to achieve a stable classification with the best classification rate.

\subsubsection{Maximum Likelihood and Bayes-Theorem}

So far, classification was mainly done in the discriminant space but there is also the possibility to classify objects without calculating the canonical discriminant functions $Y$ first. Classification can also be done by estimation of a priori probabilities and probability density functions. However, statistical classification, e.g., Maximum Likelihood or using Bayes-Theorem, can only be applied if the groups have equal covariance matrices since class relationships are estimated based on probabilities. Whether the covariance matrices are equal or not, can be tested by calculating Box's M. However, features extracted from gas sensor data are often not 
normally distributed and scatter differently for different groups [171]. Therefore, this group of classifiers is not discussed further here. Interested readers are referred to literature [230], [232], [250], [251].

\subsubsection{Fischer's Discriminant}

As mentioned earlier, LDA is sometimes seen as a classifier. This is true if instead of the canonical discriminant functions, so called simple classification functions or Fisher's (linear) discriminant is used [232]. An object is classified into the group with the highest score, i.e., highest $\mathrm{F}_{\mathrm{g}}$-value.

$$
\begin{aligned}
& F_{1}=c_{10}+c_{11} \cdot x_{1}+c_{12} \cdot x_{2}+\cdots+c_{1 N} \cdot x_{N} \\
& F_{2}=c_{20}+c_{21} \cdot x_{1}+c_{22} \cdot x_{2}+\cdots+c_{2 N} \cdot x_{N} \\
& \vdots \\
& F_{G}=c_{G 0}+c_{G 1} \cdot x_{1}+c_{G 2} \cdot x_{2}+\cdots+c_{G N} \cdot x_{N}
\end{aligned}
$$

with $G$ the number of groups and $x_{1} \ldots x_{N}$ the features. In contrast to the canonical discriminant functions, the coefficients $c_{i j}$ are calculated as:

$$
c_{g i}=(M-G) \cdot \sum_{j=1}^{N} w_{i j}^{-1} \cdot x_{j g}
$$

with $M$ the number of all objects, $N$ the number of features, $G$ the number of groups and the inverse of the within-groups sum of cross-products $w_{i j}^{-1}$. The constant term $c_{g 0}$ is calculated as follows:

$$
c_{g 0}=-0.5 \cdot \sum_{j=1}^{N} c_{g j} \cdot x_{j g}+\ln \left(P_{g}\right)
$$

with the optional term $\ln \left(P_{g}\right)$ which represents a priori probabilities which, for instance, can take different groups sizes into account [250].

It should be noted that this simple classification is only valid if the groups have equal covariance matrices. 


\subsection{Validation}

There are basically two kinds of validation: validation of the overall performance and cross-validation of the discrimination. Whereas cross-validation is a method to proof the stability of the discrimination, i.e., validation of the training model, validation is used to check the stability / quality of the whole system. For validation, a second dataset which is entirely unconnected to the training data needs to be used. For gas sensors, this could be a separate measurement or, even better, data from a second sensor. Data from the second set needs to be classified based on the parameters obtained from the first dataset, i.e., the training. If in that case a high correct classification rate can be achieved, the suggested model is stable and robust. With this approach also long-term stability can be proven.

Cross-validation of the result is an important step, especially for supervised learning methods, since they have a tendency to overestimate data; this is known as overfitting. Over-fitting in statistical learning means that there are too many parameters (here: features) for describing too few observations. Although, the outcome of the discrimination looks sufficiently good, cf. Fig. 5.6a, the value of it is rather low because the retrieved model has a poor predictive performance. That means unknown objects, even though they actually belong to one of the training groups, would not be classified correctly. Hence, the model itself does not represent the underlying relationship of the input data but overestimates noise and random errors. An example for over-fitting is shown in Fig. 5.6 where LDA was used to discriminant three classes. The data set contains 150 observations, and as features normally distributed random numbers have been used. If 145 features are used, the resulting scatter plot looks sufficiently good, cf. Fig. 5.6a, whereas with only 15 features discrimination of the three classes is not possible, cf. Fig. 5.6b.

The following elucidations are based on [216] and [265]. A simple way to test for over-fitting is cross-validation, $\mathrm{CV}$. The basic idea is to split the input dataset into at 
least two partitions. The first one is used for retrieving the model, i.e., the training, and the second one to estimate the error rate ${ }^{21}$, i.e., cross-validation.
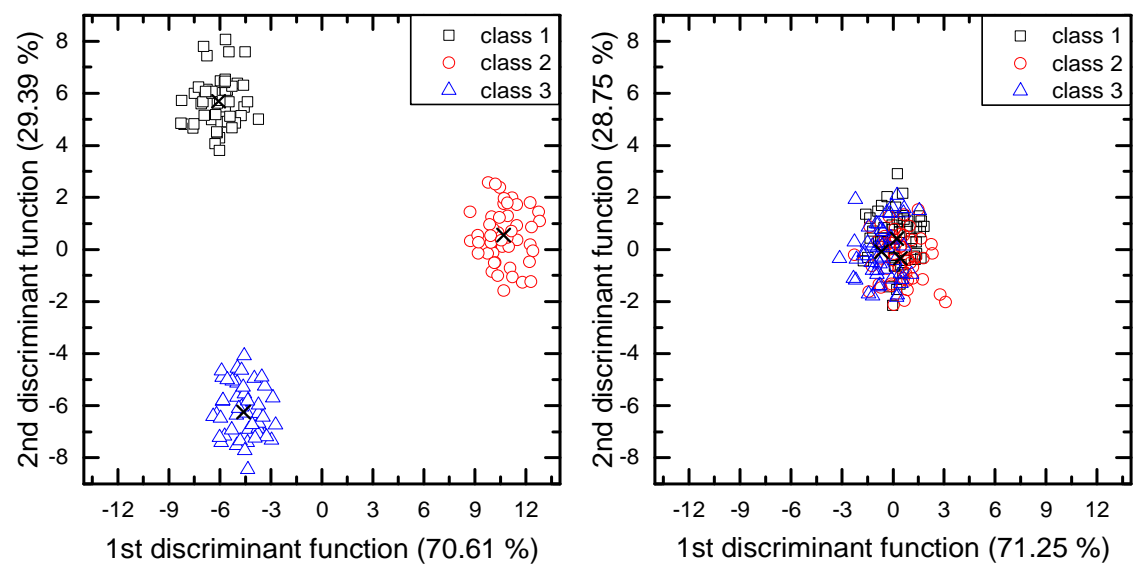

Fig. 5.6 LDA scatter plot showing over-fitting. Data set contains 150 observations (cycles) in three groups. (a) Discrimination when 145 features, and (b) only 15 features are used.

Validation-data needs to be classified based on the parameters obtained from the training. This method is known as the holdout method. The simple holdout method has basically two drawbacks when the dataset is small and depending on how the dataset is split, different results can be obtained. This can be overcome by performing multiple partitions of the dataset and averaging the performance of the model across partitions [216]. Several different algorithms for resampling the data are commonly used.

The simplest cross-validation method which can be seen as a multiple holdout method is $K$-fold cross-validation. The dataset is split into $K$ partitions whereas $K-1$ folds are used for training and a different fold for testing. Thus, $K$ experiments need to be performed and each partition is eventually used for training or testing. When the number of folds $K$ is set to the number of observations $N$, i.e., $K=N$, the method is known as leave-one-out cross-validation, LOOCV. The computational most expensive cross-validation algorithm is LOOCV, since $N$ experiments need to be performed. For K-fold cross-validation, a typical value for $K$ is 10 , and for large datasets also $K=3$ is common. Another algorithm is random subsampling. Here, $K$ splits of the entire dataset are performed. For each split a fixed, pre-defined number

\footnotetext{
${ }^{21}$ Besides the error rate also the correct classification rate is often given as a result of cross-validation.
} 
of observations (here: cycles) is selected from the dataset without replacement. The true error is the same as for K-fold cross-validation. Similar to random subsampling is bootstrap but with replacement. Thus, bootstrap preserves the a priori probability and has a higher variance in each fold than random subsampling [265], [266].

Table 3 shows the result of leave-one-out cross- validation for the LDA shown in Fig. 5.6. Different classifiers lead to slightly different correct classification rates. A correct classification rate of $100 \%$ means that every data point is classified correctly. However, the overall classification rate is in the range of a random classification (for three classes the random classification rate is $1 / 3$ ). Hence, the discrimination shown in Fig. 5.6a is just over-fitting.

Table 3 Result of leave-one-out cross-validation with either Mahalanobis distance or k-Nearest Neighbor, i.e., $\mathrm{k}=3$, classifier for LDA shown in Fig. 5.6.

\begin{tabular}{|c|c|c|c|c|c|}
\hline class & $\begin{array}{l}\text { LOOCV } \\
\text { (Mahal.) }\end{array}$ & $\begin{array}{l}\text { LOOCV } \\
(\mathrm{kNN})\end{array}$ & class & $\begin{array}{l}\text { LOOCV } \\
\text { (Mahal.) }\end{array}$ & $\begin{array}{l}\text { LOOCV } \\
(\mathrm{kNN})\end{array}$ \\
\hline overall & $30.67 \%$ & $25.33 \%$ & overall & $38.00 \%$ & $34.67 \%$ \\
\hline 1 & $32 \%$ & $26 \%$ & 1 & $30 \%$ & $36 \%$ \\
\hline 2 & $18 \%$ & $28 \%$ & 2 & $32 \%$ & $28 \%$ \\
\hline 3 & $42 \%$ & $22 \%$ & 3 & $52 \%$ & $40 \%$ \\
\hline
\end{tabular}





\section{Experimental Setup}

This chapter deals with the experimental setup, e.g., the used gas mixing systems in Section 6.1. Several different kinds of SiC-FET gas sensors are made available by the Division of Applied Sensor Science at Linköping University and SenSiC AB, Kista, Sweden and are presented briefly in Section 6.2. Control and data acquisition hardware is provided by $3 S \mathrm{GmbH}$ - Sensors, Signal Processing, Systems, Saarbrücken, Germany based on given requirements defined within this $\mathrm{PhD}$ project, and a description is given in Section 6.4. The last two sections deal with software for operating the sensor hardware and data evaluation which have been developed during this $\mathrm{PhD}$ project.

\subsection{Gas Mixing Apparatus}

Several different gas mixing systems have been used for characterization of SiC-FET gas sensors. There are two basic concepts which are discussed in the following.

\subsubsection{Standard System}

A standard gas mixing system for the characterization of sensors with test gases is shown in Fig. 6.1 using the example from the Lab for Measurement Technology. This system has been developed and extended by several thesis works. Detailed information can be found, for instance, in [267], [268], [269]. The test gases are usually supplied by gas cylinders and the test gas mixture is set by adjusting the mass flow ratio of test and carrier gas. The different flows are controlled by mass flow controllers, MFC. At the Lab for Measurement Technology MFCs from MKS Instruments Inc. series 1479 and at the Div. of Applied Sensor Science MFCs from Bronkhorst High-Tech series F-201C are currently in use. As carrier gas either synthetic air provided by a zero air generator or a mixture of oxygen and nitrogen 
from gas cylinders are typically used. The flow is usually in the order of a few hundred milliliters per minute, typically $200 \mathrm{ml} / \mathrm{min}$. Test gases are injected into the main stream by 3/2-way valves. For test gases MFCs with a maximum flow of $10 \mathrm{ml} / \mathrm{min}$ or $20 \mathrm{ml} / \mathrm{min}$ are used. The total flow over the sensor is kept constant. In order to set a level of humidity the carrier gas stream is split into a $100 \%$ dry stream and one going through a water bottle ("bubbler") which is nearly $100 \%$ humid. All MFCs and valves are controlled by a computer program.

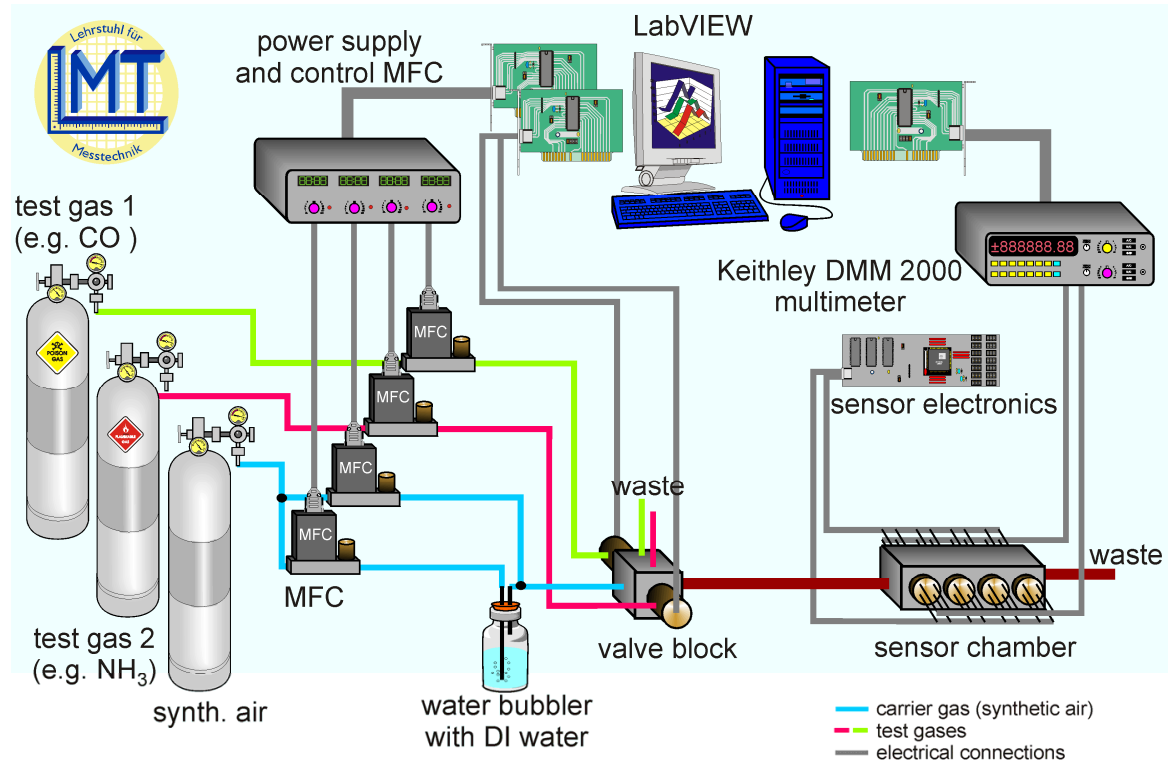

Fig. 6.1 Schematic diagram of the gas mixing system used for measurements with typical exhaust gases at the Lab for Measurement Technology.

\subsubsection{System for Volatile Organic Compounds}

For indoor air quality applications, volatile organic compounds, VOC, need to be measured at ultra-low concentrations; typically in the low parts-per-billion, ppb, range. Performing such measurements impose particular requirements on the test apparatus. Therefore a new gas mixing system, cf. Fig. 6.2, has been developed and built up at the Lab for Measurement Technology. Here, only a brief overview is given and a detailed description of the system can be found elsewhere [270]. The basic setup is similar to the standard system described earlier. However, test VOCs are supplied by either permeation tubes or gas cylinders followed by a pre-dilution 
step. Permeation ovens, VICI Dynacalibrator 150, are used for highly accurate temperature control of the tubes. The concentration of VOCs can be set by the flow through the oven, here a MFC with maximum $500 \mathrm{ml} / \mathrm{min}$ is used, and its temperature ${ }^{22}$; a typical flow is $100 \mathrm{ml} / \mathrm{min}$. This flow is further decreased by a second MFC, typically a MFC with maximum $20 \mathrm{ml} / \mathrm{min}$ is used, and the pressure between the oven and the second MFC is controlled by a mechanical inlet pressure regulator from Swagelok. Another possibility to supply low concentrations of VOCs is to use gas cylinders and a gas pre-dilution step as shown in Fig. 6.2. Humidification is again realized by splitting the carrier gas stream where one part is let through a water bubbler which temperature is stabilized at $20^{\circ} \mathrm{C}$ by a thermostat, LAUDA RE307.

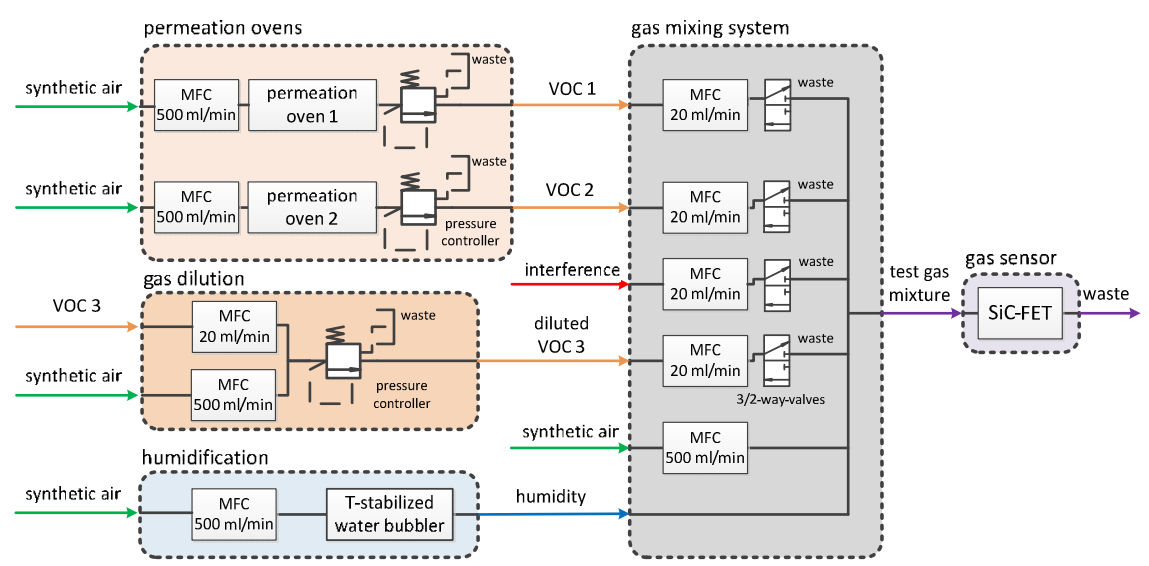

Fig. 6.2 Schematic diagram of the gas mixing system used for volatile organic compounds measurements. Adapted from [270], [47].

In all parts of the system, the same carrier gas, usually synthetic air, from the same gas cylinder is used. This is particularly important since even the most pure synthetic air commercially available with a purity of, e.g. 5.0, contains contaminations with concentrations up to a few parts-per-million, ppm. Nevertheless, when using the same carrier gas in all parts of the system, e.g., as the main stream, in the permeation oven, and for pre-dilution, these contaminations form a constant background, and thus, do not affect the sensor response. However, after exchanging the synthetic air cylinder, the baseline of the sensors shifts due to a slightly different background.

\footnotetext{
${ }^{22}$ The permeation rate depends strongly on temperature.
} 
The purity of the system has been checked by using gas chromatography-mass spectroscopy, GC-MS, reference measurements [270]. So far, an online verification during the measurements is not possible and the users have to rely on what is set by the system. However, the total flow over the sensor is monitored by a mass flow meter, MFM. 


\subsection{SiC-FET Gas Sensors}

During this $\mathrm{PhD}$ project, several different kinds of SiC-FET gas sensors developed and continuously improved at Div. of Applied Sensor Science and SenSiC AB, Kista, Sweden have been available. The design and manufacturing of the sensors is not part of this project, instead it is focused on the development of methodologies for operation and data evaluation. Further, it should be noted that not all different kinds of sensors have been available from the beginning of the project. Development of the sensors has been done simultaneously and often sensor prototypes have been provided to be used in this project. Thus, not every measurement can be done with all different kinds of sensors so that a comparison of the sensors is not possible. Nevertheless, in the following sections the different SiC-FET sensors are briefly described.

\subsubsection{Two-Terminal Depletion Type}

At the very beginning, simple two-terminal ${ }^{23}$ depletion type SiC-FETs where the gate is connected to the source are tested as shown in Fig. 6.3. As can be seen the design involves a buried channel giving rise to more stability and robustness in harsh environments but making the structure more complicated.

The device has been designed and fabricated at Linköping University together with ACREO AB, Kista, Sweden and later ASCATRON AB, Kista, Sweden. The sensors are processed on p-type $\mathrm{SiC}$ wafers and bond pads are realized by a stack of platinum and titanium. Each die holds three transistor structures with the gate connected to source on chip level as well as a test structure which is not used in this project cf. Fig. 6.3b. The chip size is $2.1 \mathrm{~mm} \times 1.9 \mathrm{~mm}$. As catalytic gate metallization, a $25 \mathrm{~nm}$ thick porous platinum film deposited by sputter-deposition in an argon atmosphere at $50 \mathrm{mTorr}$ is used.

The sensor chip is glued on a ceramic heater (Heraeus Holding GmbH, Hanau, Germany) together with a Pt-100 temperature sensor and mounted on a 16-pin TO-8

\footnotetext{
${ }^{23}$ Usually, the substrate (backside) contact is not counted and the two terminals are drain and source. Sometimes these devices are called diode structures.
} 
header as shown in Fig. 6.4. Electrical contacts between FET and header are realized via gold wire bonding. The used heater is a Pt-6.8 with outer dimension of $9.5 \mathrm{~mm} \times 1.9 \mathrm{~mm}$. The thermal time constant, $\tau_{63}$, is in the order of 2 to 3 seconds [271]. The Pt-100 temperature sensor is used as a reference sensor and to adjust the heater calibration curve. However, the measured temperature is obviously not the exact temperature of the sensitive layer.

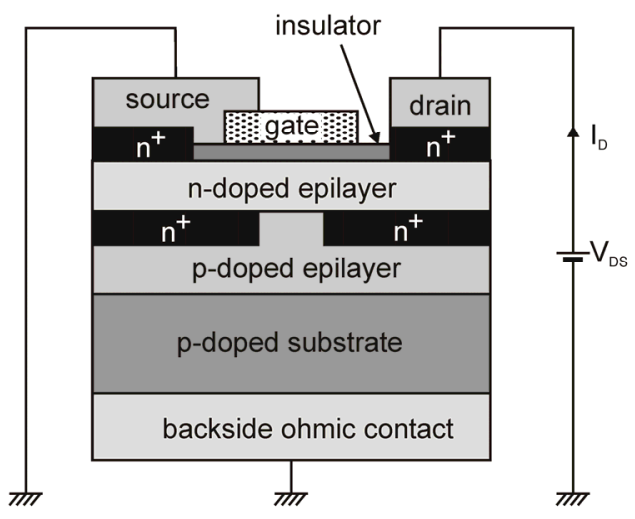

(a)

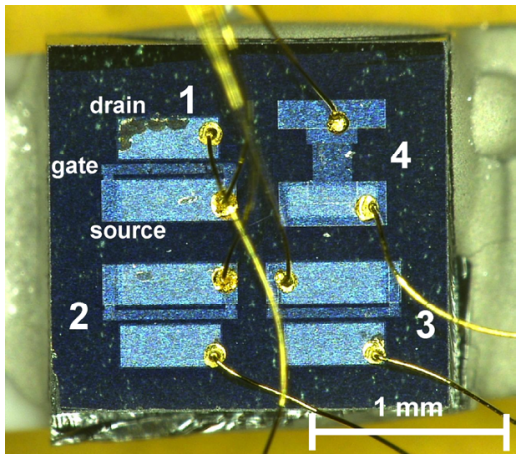

(b)

Fig. 6.3 (a) Cross sectional view of a two-terminal depletion type SiC-FET, and (b) picture of the $\mathrm{SiC}$ die holding three transistor structures (1-3) and one test structure (4).

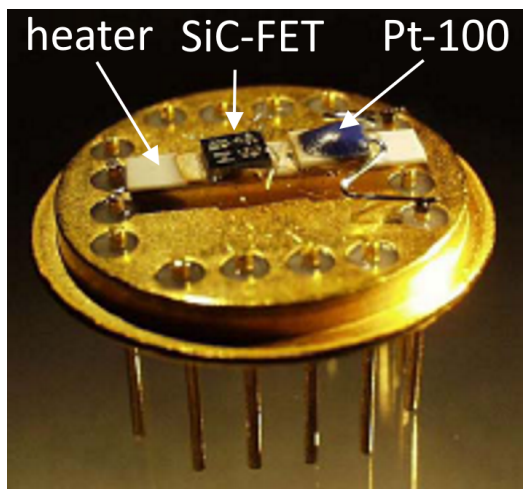

Fig. 6.4 Photograph of a SiC-FET mounted on a TO-8 header. 


\subsubsection{Two-Terminal Enhancement Type}

In addition to depletion SiC-FETs, also enhancement type SiC-FETs as shown in Fig. 6.5 were available. The gate metallization is either $25 \mathrm{~nm}$ platinum or $30 \mathrm{~nm}$ iridium. Both are deposited by sputter-deposition in an argon atmosphere at 50 mTorr. The mounting is the same as for the depletion type sensors. An exemplary IV-curve is given in Fig. 6.6 showing the influence of temperature and the response to $500 \mathrm{ppm}$ carbon monoxide, CO. Typically this type of SiC-FET is operated at a constant drain-current of, e.g., $100 \mu \mathrm{A}$. The resulting drain-source-voltage drop is recorded as the sensor signal.
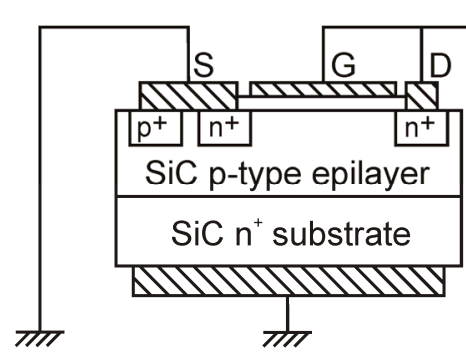

(a)

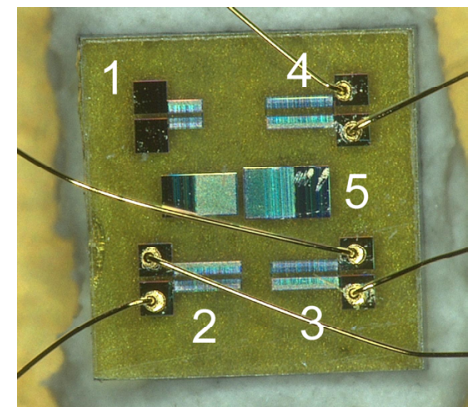

(b)

Fig. 6.5 (a) Cross sectional view of a two-terminal enhancement type SiC-FET, and (b) picture of the $\mathrm{SiC}$ die holding three transistor structures $(2-4)$ and two test structure $(1,5)$.

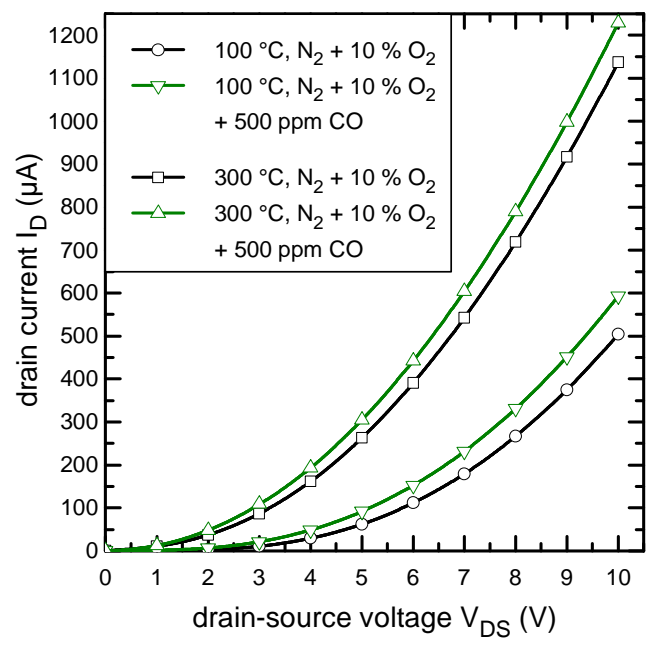

Fig. 6.6 Current-voltage, IV, curve of an enhancement type SiC-FET with iridium gate. 


\subsubsection{Three-Terminal Depletion Device}

The latest batch of sensors studied in this project involves three-terminal ${ }^{24}$ transistor devices as shown in Fig. 6.7. The sensors are commercially available through SenSiC AB, Kista, Sweden and a detailed description of the manufacturing process can be found in [272].

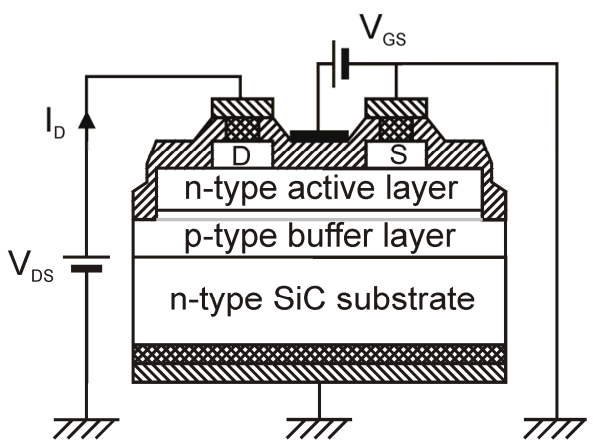

(a)

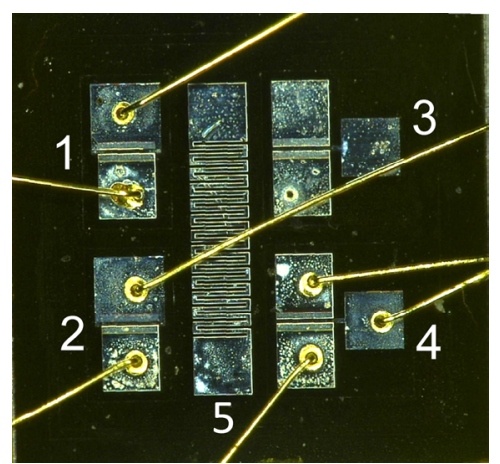

(b)

Fig. 6.7 (a) Cross sectional view of a three-terminal depletion type SiC-FET, and (b) picture of the SiC die holding two two-terminal $(1,2)$, two three-terminal transistor structures $(3,4)$ and a platinum temperature sensor $(5)$.

The catalytic gate metallization is either porous platinum or iridium with a thickness of $25 \mathrm{~nm}$ and $30 \mathrm{~nm}$, respectively.

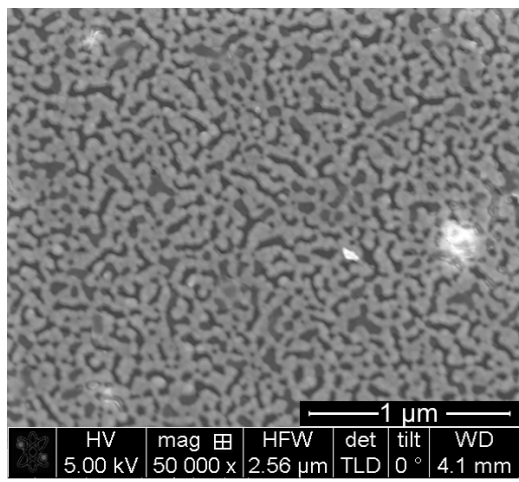

Fig. 6.8 Scanning electron microscopy, SEM, picture of a platinum gate.

\footnotetext{
${ }^{24}$ The three terminals are drain, source, and gate. The substrate contact is usually not counted in this context although, it is accessible and thus, possible to apply a non-zero bias.
} 
The porous layer including cracks that appear during deposition and annealing has a pore size in the range of $0-30 \mathrm{~nm}$ width and 0-200 nm length, cf. Fig. 6.8. The gate length is $10 \mu \mathrm{m}$ and the gate width is $300 \mu \mathrm{m}$.
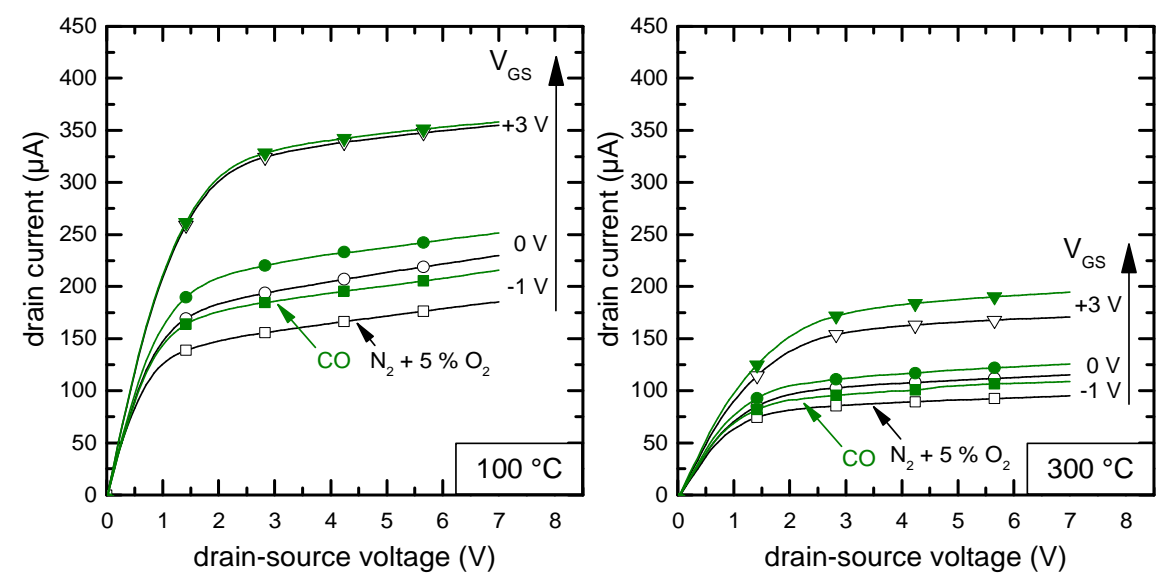

Fig. 6.9 Current-voltage, IV, curve of a three-terminal depletion type SiC-FET with platinum gate. Black open symbols mark the response in dry nitrogen with $5 \%$ oxygen and green solid symbols mark the response with additional $500 \mathrm{ppm}$ carbon monoxide. (a) At $100{ }^{\circ} \mathrm{C}$, and (b) at $300{ }^{\circ} \mathrm{C}$.

Fig. 6.9 shows exemplary current-voltage, IV, curves of a platinum gate SiC-FET. As can be seen, the saturation current decreases with increasing temperature and increases with increasing gate bias $V_{G S}$. The saturation region starts between $1 \mathrm{~V}$ and $2 \mathrm{~V}$ depending on the applied gate bias and temperature. The response to carbon monoxide, $\mathrm{CO}$, is also affected by the applied bias.

It should be noted that the value of the saturation current differs from sample to sample which does not influence the sensing behavior much. This is due the manufacturing process which is described in detail elsewhere [272]. Slightly different doping concentrations or layer thicknesses can cause differences in the saturation current. 


\subsection{Sensor Chamber}

In order to study the SiC-FET sensors in a test gas atmosphere, a sensor chamber was designed [273] with CATIA V5 R19 from Dassault Systèmes as shown in Fig. 6.10. The chamber itself has outer dimensions of $30 \mathrm{~mm}$ x $20 \mathrm{~mm}$ x $30 \mathrm{~mm}$ and holds up to three SiC-FET sensors mounted on TO- 8 headers. The body material is stainless steel which is chemically inert and the sensors are held in the chamber by small brass plates fixed by four M3 screws. The volume inside the chamber is approximately $3 \mathrm{ml}$ and all sensors have the same distance to the center of the chamber so that each sensor is exposed to same gas. Connection to the gas mixing system is done by ordinary Swagelok fittings. Fittings for both 1/8" and 1/4" Swagelok tubings are available and can be screwed in the chamber by a G1/8" ISO thread.

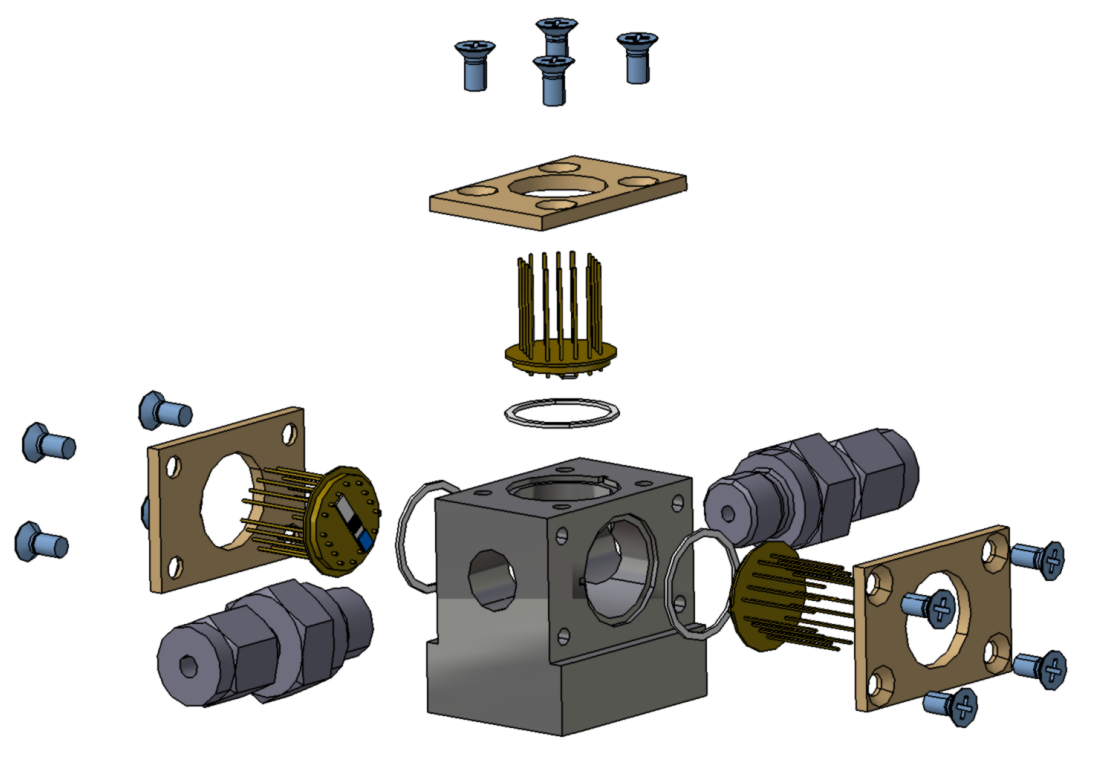

Fig. 6.10 Exploded view drawing of the sensor chamber. All parts, e.g., sensors, seals, Swagelok fittings, are shown. 


\subsection{Measurement Hardware}

Based on the experiences from earlier work [271], [273], specific requirements for a new control and read-out hardware of SiC-FETs have been defined at the beginning of this $\mathrm{PhD}$ project, cf. Table 4. Then, a dedicated hardware platform to control the sensor and to acquire data has been set up by $3 S \mathrm{GmbH}$ - Sensors, Signal Processing, Systems, Saarbrücken, Germany ${ }^{25}$. The platform is able to switch between a constant current and a constant voltage mode as well as between zero gate and / or substrate bias and non-zero bias operation. Due to the fact that several different kinds of FET sensors are used, minor adjustments (defined during this project) to the combined hardware platform are made for each new batch of sensors.

Table 4 Requirements for control and read-out hardware.

\begin{tabular}{lrr}
\hline parameter & measurement range & resolution \\
\hline Pt-6.8 heater temperature (set) & $40{ }^{\circ} \mathrm{C}-550{ }^{\circ} \mathrm{C}$ & $<3{ }^{\circ} \mathrm{C}$ \\
Pt-100 temperature sensor (read) & $40{ }^{\circ} \mathrm{C}-550{ }^{\circ} \mathrm{C}$ & $1{ }^{\circ} \mathrm{C}$ \\
drain-source voltage $V_{D S}$ (read) & $0 \mathrm{~V}-6 \mathrm{~V}$ & $100 \mathrm{mV}$ \\
drain-source voltage $V_{D S}$ (set) & $0 \mathrm{~V}-6 \mathrm{~V}$ & $<1 \mu \mathrm{A}$ \\
drain current $I_{D}$ (read) & $0 \mu \mathrm{A}-1 \mathrm{~mA}$ & $<3 \mu \mathrm{A}$ \\
drain current $I_{D}$ (set) & $0 \mu \mathrm{A}-500 \mu \mathrm{A}$ & $100 \mathrm{mV}$ \\
gate bias $V_{G S}($ set) & $-5 \mathrm{~V}-5 \mathrm{~V}$ & $100 \mathrm{mV}$ \\
substrate bias $V_{B S}(\mathrm{set})$ & $-5 \mathrm{~V}-5 \mathrm{~V}$ & $20 \mathrm{~Hz}$ \\
sampling rate & --- &
\end{tabular}

The current system controls the sensor temperature with an analog control circuit with a resolution of $1{ }^{\circ} \mathrm{C}$ and can adjust $V_{G S}$ and / or $V_{B S}$ independently from $-7 \mathrm{~V}$ to $+7 \mathrm{~V}$ using an 8 bit digital analog converter, DAC, i.e., with a resolution of approximately $50 \mathrm{mV}$. Most important for the subsequent data acquisition, the system allows precise synchronization of temperature and gate bias cycles with data acquisition. Data acquisition in case of constant voltage mode is performed by a

\footnotetext{
${ }^{25}$ The development of the hardware and its circuits are not part of this project. However, the definition of the requirements and all the functionality of the hardware as well as making minor adjustments to the hardware is part this PhD project.
} 
10 bit analog digital converter, $\mathrm{ADC}$, for measuring $I_{D}$ with a measurement range of $1-1,000 \mu \mathrm{A}$, resulting in a resolution of approximately $1 \mu \mathrm{A}$. The $I_{D}$ can be set with an accuracy of less than $1 \mu \mathrm{A}$. Data acquisition in case of constant current mode is performed by a 14 bit ADC for measuring $V_{D S}$ in a range of $0-6 \mathrm{~V}$, resulting in a theoretical resolution of approximately $0.4 \mathrm{mV}$. A typical data acquisition rate of $10 \mathrm{~Hz}$ was used. 


\subsection{Software for Control and Data Acquisition}

In order to control the hardware platform, dedicated software is developed with LabVIEW 2009 SP1 from National Instruments during this PhD project. Together with the hardware, only subVIs (virtual instrument subroutine) representing the interface to the hardware has been developed and provided by $3 \mathrm{~S} \mathrm{GmbH}$ - Sensors, Signal Processing, Systems ${ }^{26}$. As part of this $\mathrm{PhD}$ project, the main control VI (virtual instrument), which among others allows defining temperature and / or gatebias cycles and visualizes recorded signals, is developed and briefly presented in the following.

The front panel of the main VI consists of four tabs (cf. Fig. 6.11 - Fig. 6.14). On the first one, general settings (cf. Fig. 6.11 - Fig. 6.14), the operation mode (constant current or voltage) can be chosen as well as general settings can be made such as how the data should be saved, folder for measurement data, measurement time, and which hardware platform is used. Data can be saved in two different ways: as an array, i.e., matrix, in which each cycle is saved in a separate row, i.e., the columns of the matrix contain data points of the cycles. Here, only values of the drain current or drain-source voltage, depending on the chosen operating mode, are contained in the file. In addition, selected parameters can be saved on a time basis. Each row contains different parameters such as drain current, drain-source voltage, gate bias, temperature, etc. with an exact time stamp in the first (relative time) and second (real time) column. The second tab, temperature cycle (cf. Fig. 6.12), deals with the operating temperature of the sensor. Here, a temperature cycle can be defined by using temperature plateaus, ramps, or even cycles with a sinusoidal shape. On the next tab gate and/or bias cycles can be defined in a similar way as temperature cycles on the previous tab. If a zero-bias should be applied to the sensor, a relais on the hardware board can be switched which connects the gate and/or substrate contact to common ground, i.e., disconnects the voltage source from the sensor. On the last tab, cf. Fig. 6.14, diagrams displaying drain current and drain-source voltage (top panel) as well as the applied cycles (bottom panel) are drawn over time.

\footnotetext{
${ }^{26}$ These subVIs are needed to communicate with the hardware. The program (mainVI) to control and acquire sensor data is developed as part of this $\mathrm{PhD}$ project.
} 


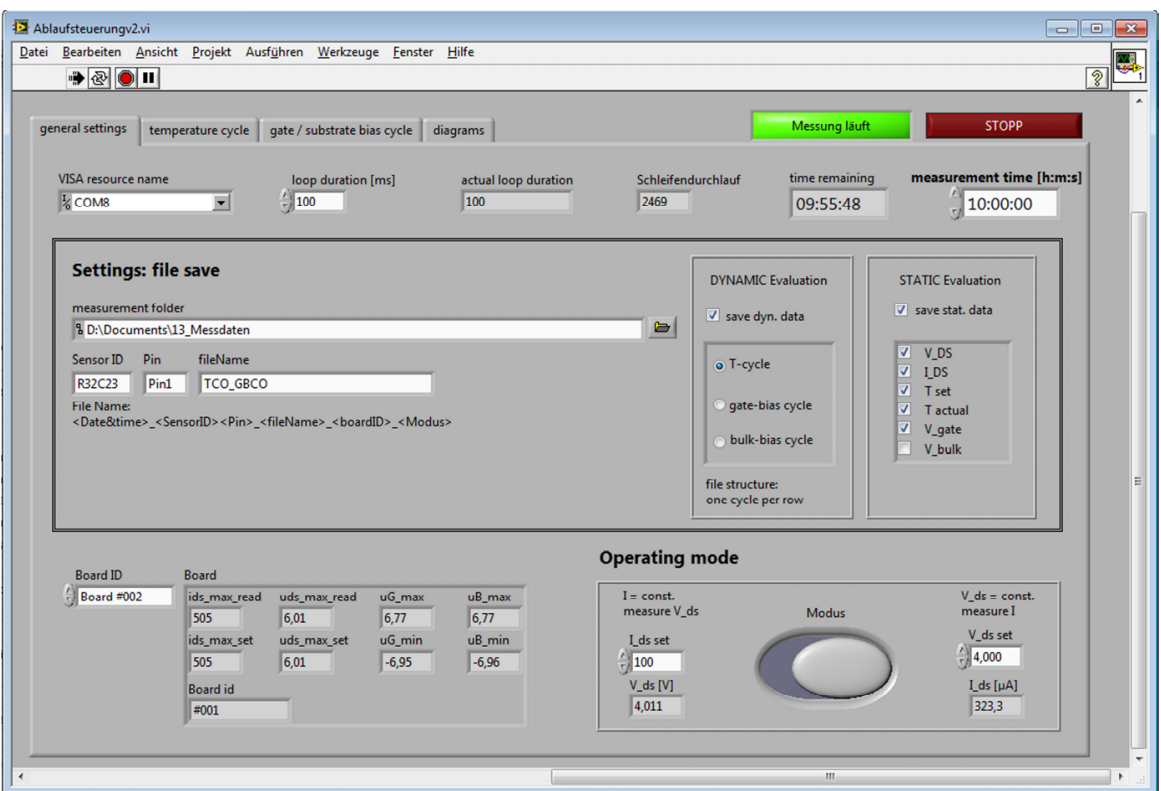

Fig. 6.11 Front panel of the control and data acquisition software tab general settings.

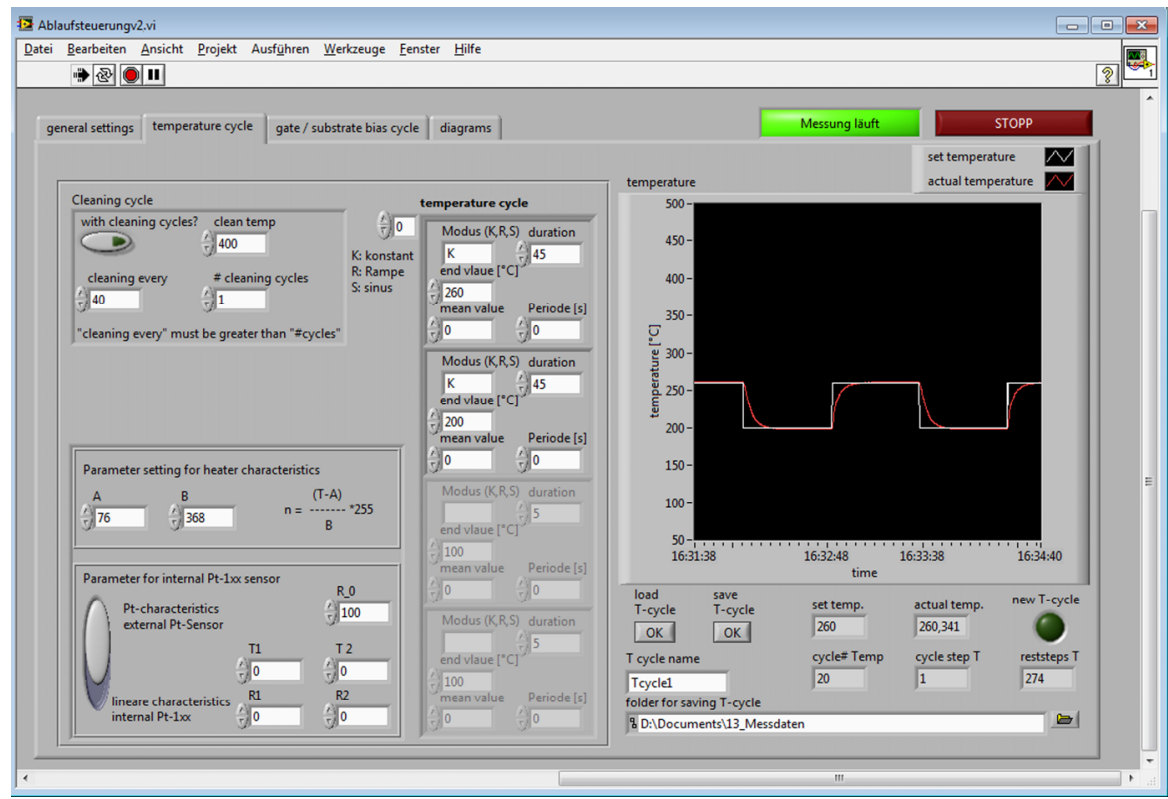

Fig. 6.12 Front panel of the control and data acquisition software tab temperature cycle. 


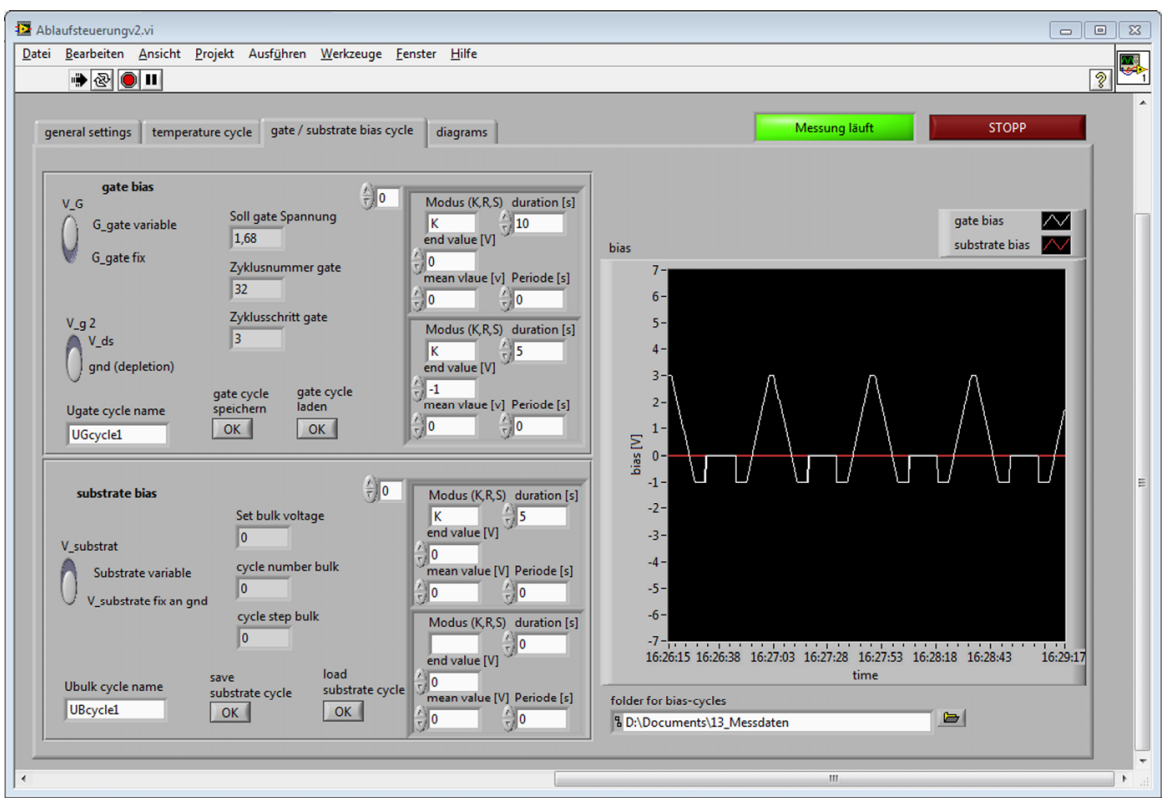

Fig. 6.13 Front panel of the control and data acquisition software tab gate / substrate bias cycle.

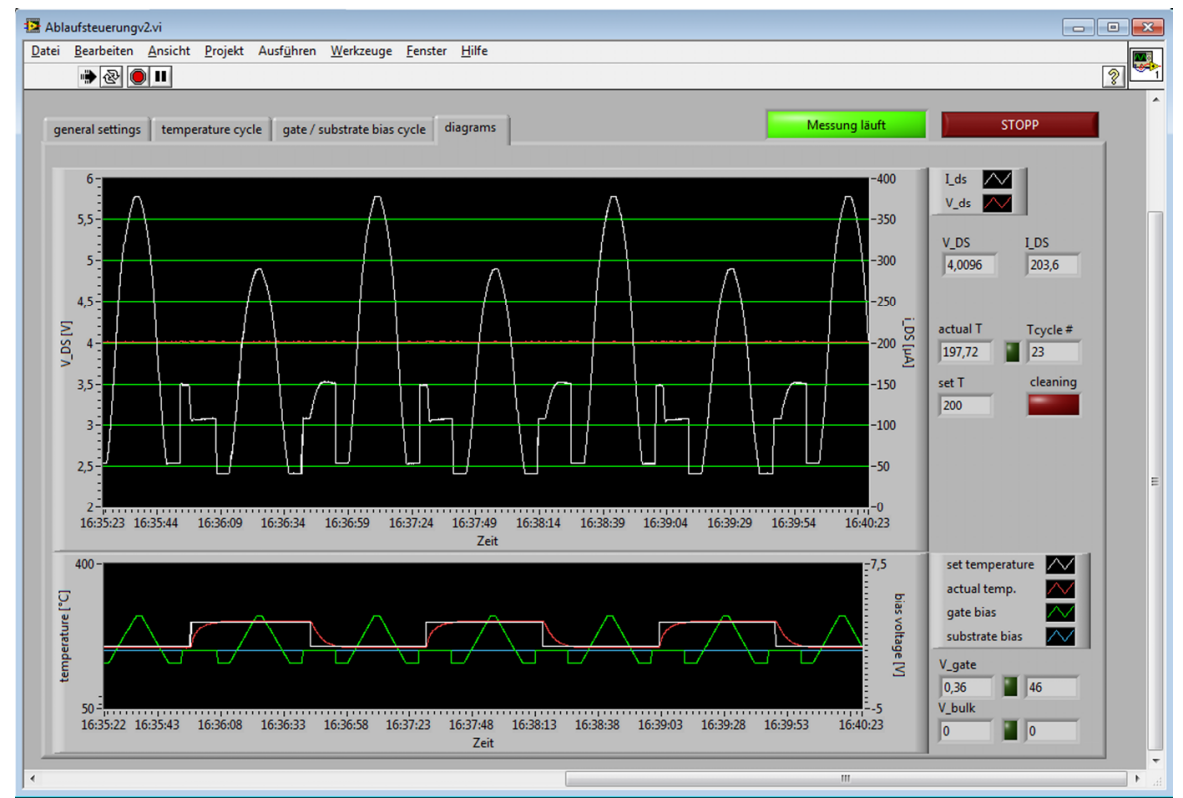

Fig. 6.14 Front panel of the control and data acquisition software tab diagrams. 


\subsection{Software for Data Evaluation}

For data evaluation purposes, there are several tools available at the Lab for Measurement Technology. Although, there are many commercial statistics programs on the market, like SPSS from IBM or STASTICA from StatSoft, the algorithms described in Chapter 5 are implemented in MATLAB tools based on native MATLAB functions mainly from the Statistics Toolbox [274]. Developing your own implementation has the advantage that the programs can be tailored to the area of gas sensing in terms of easy data handling and visualization.

For pre-processing and feature extraction a dedicated graphical user interface, GUI, $F E-G U I^{27}$, is developed. The output of this program, basically the feature matrix, can then be used in several different programs for evaluation, like SVM-GUI (support vector machines graphical user interface $)^{28}$ [275], Regress-GUI (linear regression and support vector regression graphical user interface $)^{29}$ [276] or, LDA-PCA-Tool. The latter one is greatly improved and extended in this $\mathrm{PhD}$ project, cf. Table 5, and thus, is described in more detail. The main improvements of the already existing GUI are the use of native MATLAB functions, several additional plots, the implementation of cross-validation, different classifiers, and various statistical values. The graphical user interface of the LDA-PCA-Tool is shown in Fig. 6.15. Main settings, e.g., the algorithm, features, and Ansatz-functions to be used, are made on the left hand side of the GUI (labels 1, 2, and 4). Additional plots and special settings can be made in the center (label 3). On the right hand side, results of the discrimination, i.e., statistics for each feature, the entire discrimination, and each principal component or discriminant function, are given. All plots will be displayed in separate windows.

\footnotetext{
${ }^{27}$ The FE-GUI has been developed as part of a seminar work which has been supervised by me.

${ }^{28}$ The SVM-GUI has been developed as part of a bachelor thesis which has been supervised by me.

${ }^{29}$ The Regress-GUI has been developed as part of a master thesis which has been supervised by me.
} 
Table 5 Overview of software tools at the Lab for Measurement Technology.

\begin{tabular}{|c|c|c|}
\hline software tool & available beforehand & developed during this project \\
\hline $\begin{array}{l}\text { pre-processing } \\
\text { feature } \\
\text { extraction }\end{array}$ & $\begin{array}{l}\text { MATLAB scripts, any adjustments to } \\
\text { the given problem need to be made in } \\
\text { the code }\end{array}$ & $\begin{array}{l}\text { FE-GUI } \\
\text { - } \text { convenient and easy data } \\
\text { - dandling } \\
\text { - } \quad \text { various normalizations } \\
\text { - } \text { smoothing algorithms } \\
\text { - } \quad \text { various features } \\
\text { - feature pre-selection } \\
\text { - } \quad \text { possibility to add new } \\
\text { functions }\end{array}$ \\
\hline LDA / PCA & $\begin{array}{l}\text { Simple MATLAB GUI } \\
\qquad \quad \text { PCA, LDA, and stepwise } \\
\text { LDA (own implementation) } \\
\bullet \quad \text { Only scatter plot } \\
\text { Extra tool for projection of new data }\end{array}$ & 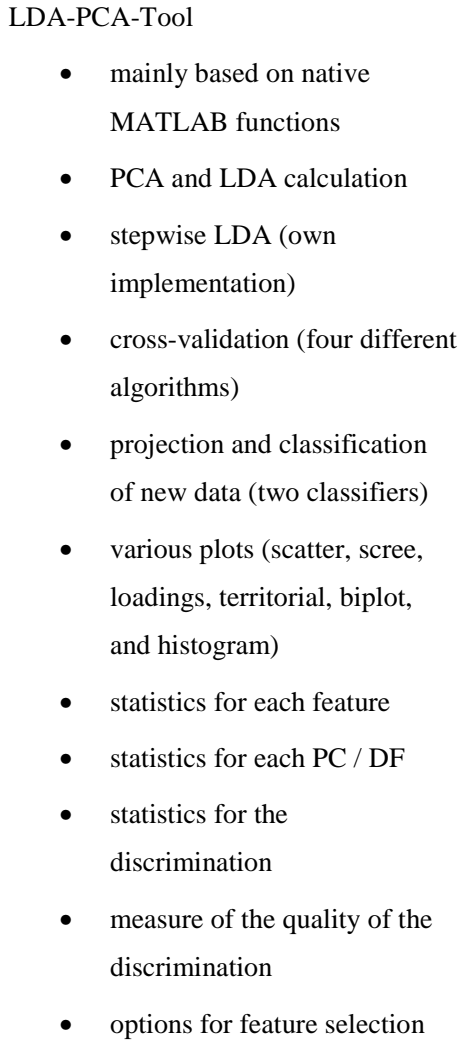 \\
\hline $\begin{array}{l}\text { Regression } \\
\text { Analysis }\end{array}$ & --- & Regress-GUI [276] \\
\hline SVM & --- & SVM-GUI [275] \\
\hline
\end{tabular}




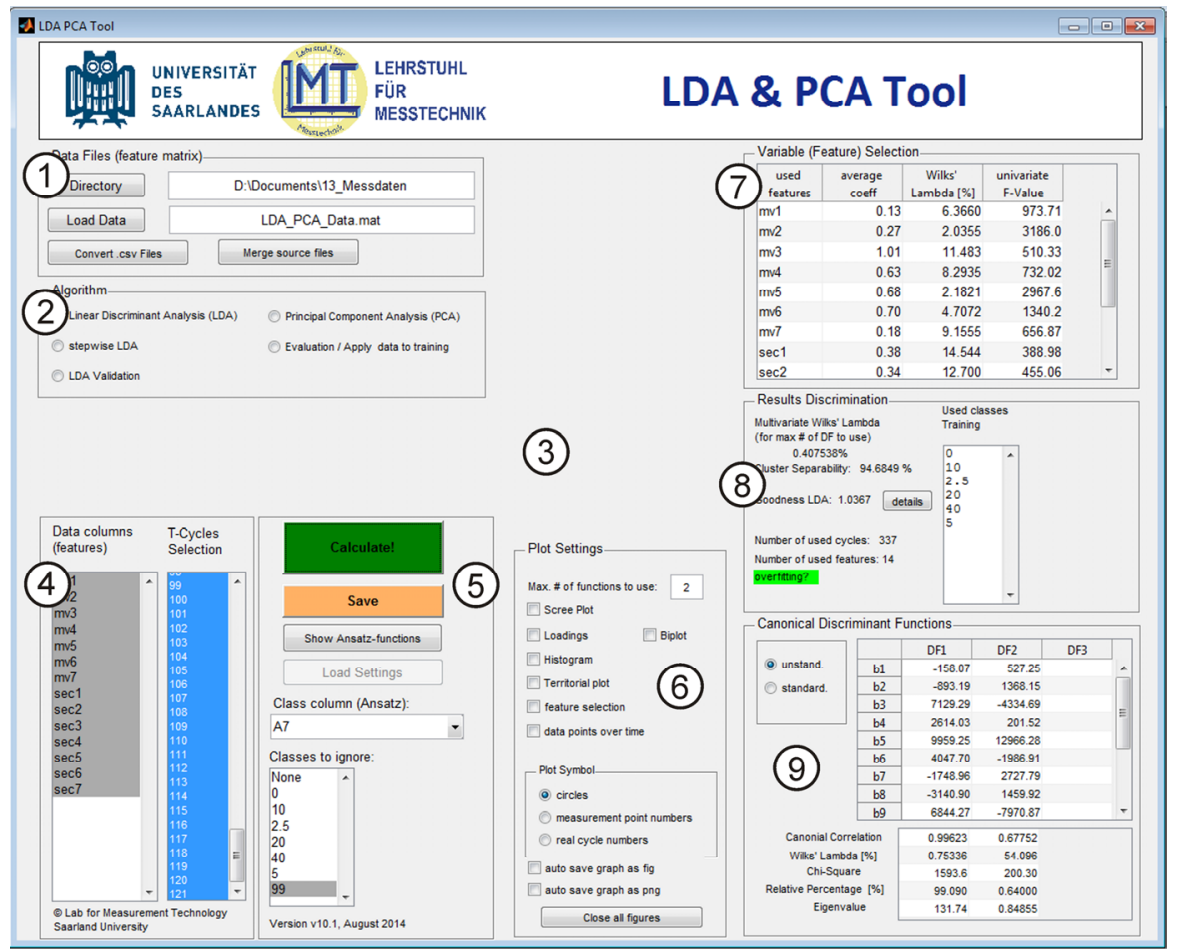

Fig. 6.15 Graphical user interface of the LDA-PCA-Tool.

After a file with pre-processed data is loaded, cf. Fig. 6.15 label 1, an algorithm, e.g., LDA can be chosen (label 2). Depending on the chosen algorithm, further control elements will appear on the GUI and additional settings can be made (label 3). After that, features need to be selected (label 4) which are used for calculation later on as well as a suitable Ansatz-function (label 5). An Ansatz-function basically defines different classes in the data set which are to be separated by the chosen algorithm. Usually, a scatter plot is shown after each calculation but additional plots like a scree-plot, loadings plot, biplot, territorial plot, or histograms as described in Section 5.3 can be selected, too (label 6). The implemented LDA algorithm is based on a native MATLAB function, manova1 [277] which does one-way multivariate analysis of variance. The output of the function provides the eigenvectors and eigenvalues for the problem given in Eq. (5.21) as well as some statistics. For PCA the native MATLAB function pca [278] is used. Besides the scatter plot, statistics for each feature, e.g., the average coefficient, the univarite $F$-value, and univariate Wilks' Lambda, which can among others be used for feature selection, are 
provided (label 7). The quality of the entire discrimination can be determined by, e.g. the multivariate Wilks' Lambda and the cluster separability, label 8. Statistical values for each principal component or discriminant function are also displayed on the GUI, label 9.

Since LDA as a supervised learning method has a tendency to over-fitting, crossvalidation, $\mathrm{CV}$, algorithms as described in Section 5.5 are implemented. Partitioning of the data which is the key step in $\mathrm{CV}$ is based on the native MATLAB functions cvpartition [279] for LOOCV and K-fold cross-validation, and datasample [280] for random subsampling and bootstrap.

Projection of new data points into the scatter plot of the training data is easily possible by choosing the algorithm evaluation. Optionally, a classifier can be applied to determine the correct classification rate as well as the confusion matrix. Classification can either be done using Mahalanobis distance classifier, mahal [281], or k-Nearest Neighbors, knnsearch [282].

The implemented stepwise LDA method is a combination of both the forward selection and backward elimination techniques in order to find the most important features by using the F-value criteria as described in [250].

The LDA-PCA-GUI is basis for most of the data evaluation and signal processing which is used in this thesis to obtain the results presented in Chapters 7 and 8. It offers every statistical parameter and method associated with Linear Discriminant Analysis introduced in Chapter 5. 



\section{Methodology Dynamic Operation}

In this chapter, different operating modes for gas sensitive field effect transistors are briefly discussed. Further, the methodology of dynamic operation in terms of temperature modulation and gate bias modulation is introduced which are the basis for results presented in Chapter 8 .

\subsection{Basic Sensor Operating Modes}

Since field effect transistors in the most general case are four-terminal devices (drain, source, gate, and substrate contact $)^{30}$, several operating modes, such as constant drain current or constant drain-source voltage, are conceivable. As discussed in Section 3.1, a standard MISFET has two basic operating regions depending on the gate bias: the linear and the saturation region, cf. Fig. 3.9. Thus, the first question to deal with is in which region should the transistor, i.e., the sensor be operated. From a theoretical point of view, the saturation region should be favored since, as discussed earlier, cf. Section 3.4, gas interaction with the sensor changes the threshold voltage of the device or, in other words, gas interaction gives rise to an internal gate bias in addition to the externally applied bias. For the drain current in the linear region the following relation can be derived:

$$
I_{D}=\frac{K}{2} \cdot\left(V_{G S_{\text {external }}}+V_{G S_{\text {internal }}}-V_{t h}\right) \cdot V_{D S}
$$

and for the saturation region:

$$
I_{D}=\frac{K}{2} \cdot\left(V_{G S_{\text {external }}}+V_{G S_{\text {internal }}}-V_{t h}\right)^{2}
$$

\footnotetext{
${ }^{30}$ In this thesis, the substrate contact is neglected and the term three-terminal device is used when all terminals are accessible and the term two-terminal device is used if the gate is connected to either drain or source.
} 
From the two equations above it is obvious that the saturation region gives rise to larger sensor response due to the fact that $V_{G S_{\text {internal }}}$ contributes to the second power. However, the IV-curve of a MISFET is almost flat in the saturation region which calls for constant drain-source voltage $\left(V_{D S}=\right.$ const.) operation where the drain current $I_{D}$ is recorded as the sensor signal. Not only the gate bias has an influence on the IV-curve but also the temperature which indeed shifts the IV-curve. Especially, when the temperature is modulated, the operating point of the transistor needs to be chosen carefully.

In the following, different operating modes and their advantages and disadvantages for certain applications are briefly discussed.

\subsubsection{Constant Drain Current}

The standard operating mode for two-terminal devices is measuring drain-source voltage while the current is kept constant, cf. Fig. 7.1a. It was reported that this operation is much more long-term stable as compared to the use of a constant drainsource voltage [90]. Additionally, it is often more convenient to measure voltage with high resolution than current. In case of a two-terminal device, the gate is connected to either drain, i.e., depletion type FET, or source, i.e., enhancement type FET, which leads to the fact that the transistor is always operated at the onset of saturation, cf. Eq. (7.2).
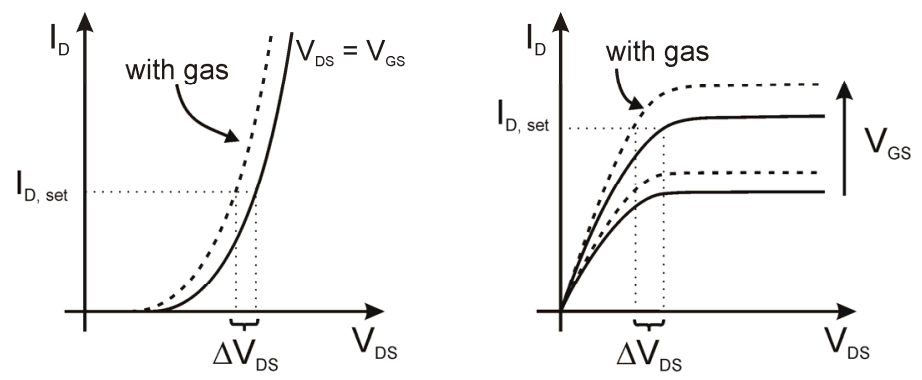

Fig. 7.1 Schematic explanation of constant drain current operating mode. (a) For a two-terminal device where gate is connected to drain, and (b) for a three terminal device.

In contrast to that, three terminal devices can only be operated with a constant drain current in the linear region since the IV-curve is almost flat in saturation. Since $I_{D}$ 
depends on $V_{D S}$ in the linear region, Eq. (7.1), it is obvious that a higher response can be obtained at the end of the linear region, i.e., at the onset of saturation. For dynamic operation, i.e., either temperature and/or gate bias is modulated, this operating mode is inconvenient to use. As shown in Fig. 7.1b, the influence of the gate bias on the IV-curve is much higher than the shift due to gas reaction. The same is valid for temperature but in the opposite direction, i.e., increasing temperature lowers the IV-curve. Hence, when modulating temperature or bias while drain current is kept constant, the sensor can leave the linear region. Since the IV-curve is flat in saturation, the measured drain-source voltage would easily be out of range.

\subsubsection{Constant Drain-Source Voltage}

Besides the drain current, also the drain-source voltage can be kept constant which is possible in both transistor regions, cf. Fig. 7.2. However, as discussed earlier operating in saturation is the favored regime due to an increased sensor response, cf. Fig. 7.2b. When measuring in saturation with three-terminal devices, the drainsource voltage needs to be kept constant and the change in drain current is then recorded as sensor signal. Usually, the operating point is chosen in deep saturation, typically $V_{D S}=4 \mathrm{~V}$, especially when changing either temperature and / or bias. For two-terminal devices constant current mode is preferred due to the reported stability issues [90].
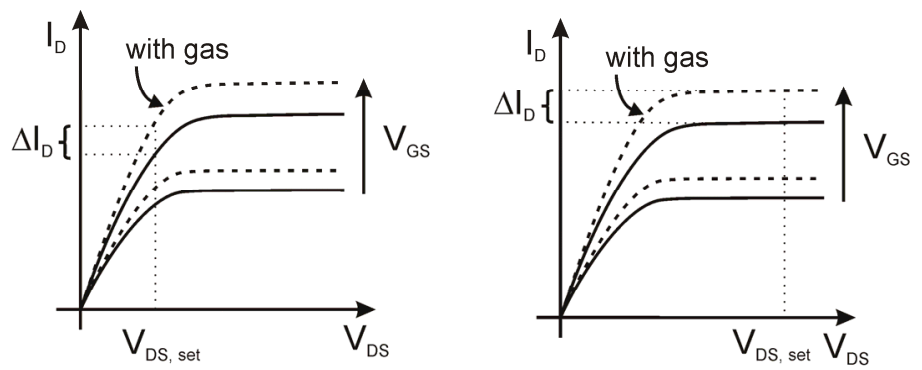

Fig. 7.2 Schematic explanation of constant drain-source voltage operating mode. (a) In the linear region, and (b) in saturation region. 


\subsubsection{Adjusting the Gate Bias}

For three-terminal devices, there is also a third option. Both drain current and drainsource voltage are kept constant. The change in IV-curve due to gas reaction or change in temperature is compensated by adjusting the applied gate bias. Hence, the sensor response is the change in gate bias $\Delta V_{G S}$. In principle, this operating mode measures directly the change in threshold voltage of the device. However, since the gate bias is changed continuously in this mode, it takes away the option to modulate this parameter by intention. Therefore, this operating mode is more scientifically relevant for studying the sensing mechanism on the surface. It cannot be used to enhance the selectivity or to study the influence of the applied gate bias.

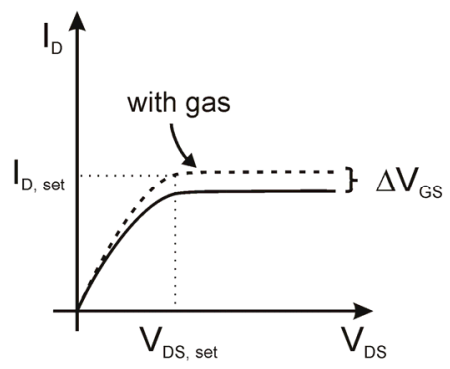

Fig. 7.3 Schematic explanation of constant operation mode, respectively constant drain current and constant drain-source voltage by adjusting the gate bias.

\subsubsection{Conclusion Sensor Operating Mode}

The choice of an operating mode is a crucial point and depends on the specific circumstances such as which kind of FET sensor is used and if it is run in static or dynamic operation. For two-terminal devices, constant current mode should be chosen due to the increased stability. This is valid for constant temperature operation as well as for temperature modulation. In case of three-terminal devices, the sensor should be operated in saturation especially, if temperature and/or bias are modulated. Then, constant voltage mode needs to be chosen where the drain current is measured. However, in some cases where a constant current is explicitly wanted or if current cannot be measured with high resolution, the sensor can be operated at the end of the linear region, i.e., at the onset of saturation, in constant current mode. 


\subsection{Temperature Modulation of SiC-FETs}

In this sub-chapter, the main idea of temperature modulation on field effect transistors is presented which is the basis for the result chapter. This section is based on a conference contribution [283] and a journal article [246] in which temperature cycled operation, TCO, on SiC-FETs are suggested for the first time. For these measurements, a depletion type SiC-FET as shown in Fig. 6.5 is used.

\subsubsection{Temperature Cycle and Feature Extraction}

When choosing a temperature cycle for an application, there are some aspects which need to be considered. Usually, the cycle should cover a broad temperature range in order to be selective to a variety of gases. This requirement is often in conflict with an acceptable cycle length. Since the concentration of the test gas needs to be constant during a cycle, there is a strong need to design rather short cycles. For sensors with long thermal time constants, for instance due to a thick substrate, typical cycle lengths are in the range of 20 to 90 seconds whereas for thin film or membrane sensors cycles are often not longer than a few seconds. Not only the length but also the shape of the cycle is important. For applications where the ambient temperature and/or flow changes different temperatures covered by the cycle should be approached by ramps rather than sharp steps. By using sufficiently slow ramps, the temperature of the sensor is always under control and thus, less sensitive to changes in the ambient. This is especially important for cooling down after a high temperature phase. However, often a lot of information is achieved when the temperature changes rapidly.

In order to study if temperature modulation is generally possible for gas sensitive field effect transistors, a rather simple cycle shown in Fig. 7.4a is used. The cycle consists of four temperature plateaus covering a temperature range from $100{ }^{\circ} \mathrm{C}$ to $330{ }^{\circ} \mathrm{C}$. This cycle is used for measuring typical exhaust gases, e.g., nitrogen monoxide, $\mathrm{NO}$, carbon monoxide, $\mathrm{CO}$, propene, $\mathrm{C}_{3} \mathrm{H}_{6}$ as a hydrocarbon, as well as ammonia, $\mathrm{NH}_{3}$ which is of interest in selective catalytic reduction, $\mathrm{SCR}$, systems where urea is injected to the exhaust in order to reduce nitrogen oxides. Additionally, hydrogen, $\mathrm{H}_{2}$, is used as well since the first FET gas sensor was introduced as a 
hydrogen sensor. In Fig. 7.4b the dynamic sensor response which basically shows how the shape of the response is altered during gas exposure is given.
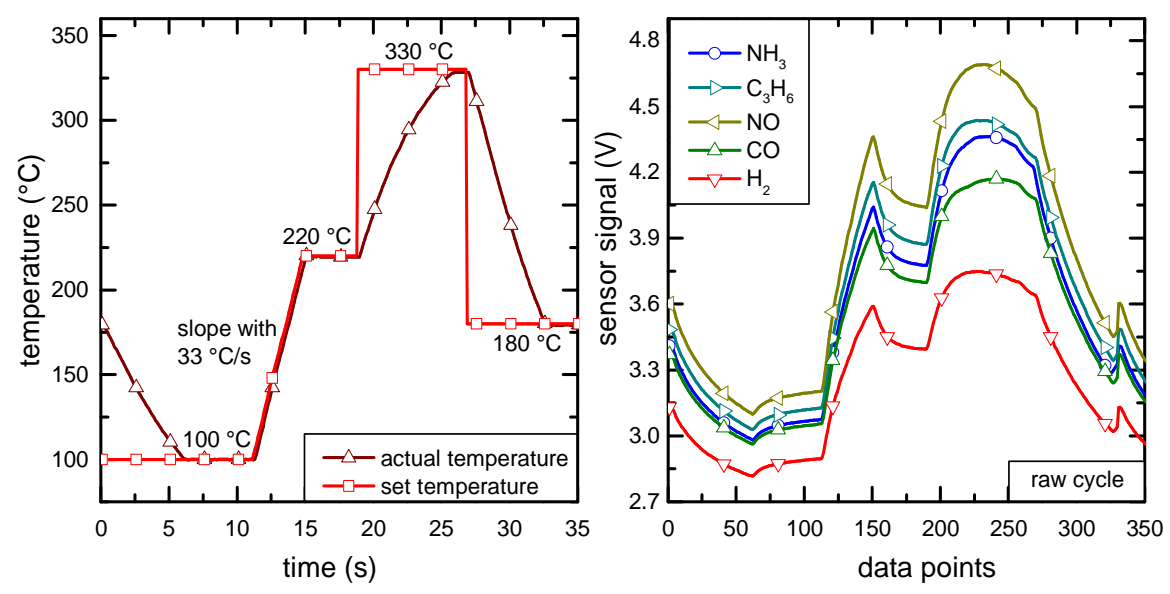

Fig. $7.4 \quad$ (a) Applied temperature cycle showing the set temperature (red) and actual temperature measured by the additional Pt-100 (brown). Adopted from [283], and (b) dynamic sensor response for $25 \mathrm{ppm} \mathrm{NH}_{3}, 75 \mathrm{ppm} \mathrm{C}_{3} \mathrm{H}_{6}, 100 \mathrm{ppm} \mathrm{NO}, 400 \mathrm{ppm} \mathrm{CO}$, and $400 \mathrm{ppm}_{2}$ in dry nitrogen.

\subsubsection{Pre-Processing and Feature Extraction}

In order to reduce the influence of drift, the data are normalized first. In temperature cycled operation, TCO, normalization is done on a cyclic basis which means that each cycle is normalized individually. Due to the fact that normalization stresses certain parts of a cycle whereas other parts are highlighted, cf. Section 5.2.1, the applied normalization should carefully be chosen. Since in this study, the methodology of TCO is applied to FET sensors for the first time, normalization strategies known from MOX sensors are used. The most commonly used normalization for metal oxide sensors is setting the cycle mean to one.

The corresponding normalized dynamic sensor response is given in Fig. 7.5a. The differences in sensor response of the gases are reduced as compared to the raw signal, cf. Fig. 7.4b, but still significant. It is a typical phenomenon that normalization can reduce sensor drift but at the same time some information is lost. In general, for SiC-FET sensors the influence of temperature on the sensor signal is much stronger than the change due to gas reaction. This makes the definition of 
intervals where features are extracted sometimes difficult. Therefore, it can be useful to look at the difference sensor signal as shown in Fig. 7.5b. First, the sensor response is normalized, e.g., by setting the cycle mean to one, and then an average reference cycle is subtracted from each cycle. Typically, the reference is the background, i.e., carrier gas but not necessarily.

The difference (normalized) sensor response is calculated as

$$
\Delta V_{D S}=V_{D S}^{\text {normalized }}(\text { gas })-V_{D S}^{\text {normalized }}(\text { reference })
$$

By doing so, the changes due to gas reaction are highlighted while the influence of changing operating temperature or applied gate bias is vanished making the definition of the intervals much easier.
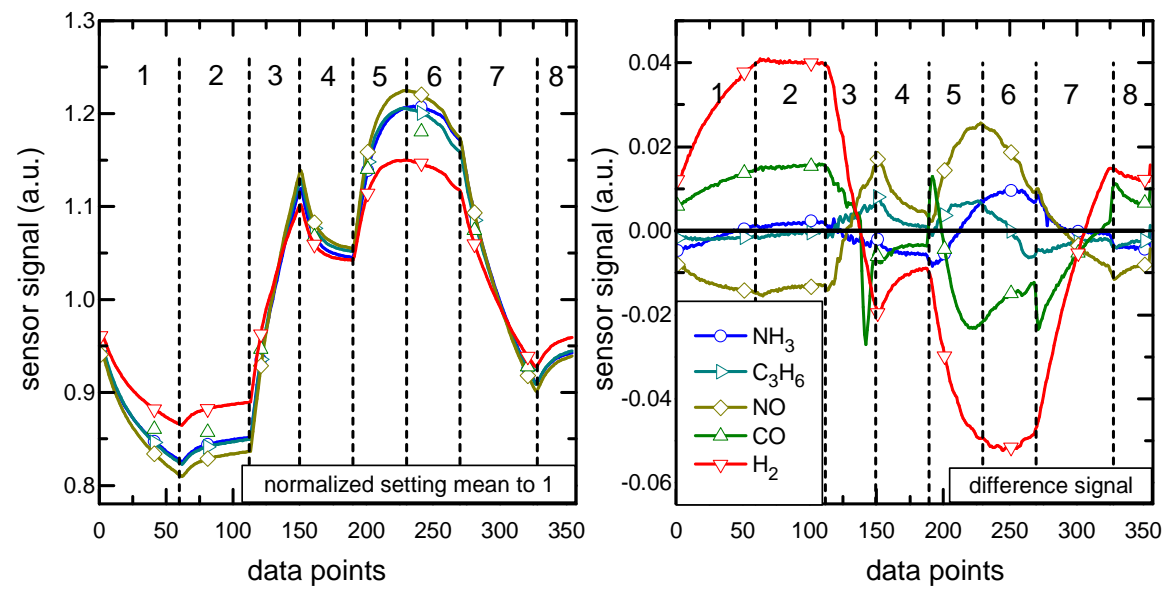

Fig. 7.5 (a) Normalized dynamic sensor response when setting the cycle mean to one with feature intervals marked adopted from [283], and (b) difference (normalized) sensor response.

In order to discriminate between the chosen gases by using pattern recognition, it is essential to extract features first. It is possible to use the sensor response itself as input data for pattern recognition but in supervised learning, e.g., LDA, there is always the issue of over-fitting. Thus, the dimension of the input data needs to be small compared to the number of observations. As mentioned earlier, there are several ways to extract features and one of the most promising techniques is to use shape-describing features, for instance, slopes and mean values in selected parts of 
the cycle. Therefore, the cycle is divided into, e.g., eight intervals as shown in Fig. 7.5. In each of these intervals the slope and the mean of the sensor response is calculated and extracted as features. These features are then used in pattern recognition afterwards for discrimination purposes. For discrimination it does not matter if features like mean values and slopes are calculated from the normalized sensor response, cf. Fig. 7.5a, or from the difference signal after normalization, cf. Fig. 7.5b.

\subsubsection{Discrimination and Quantification}

After the extraction of features from the dynamic sensor response, discrimination is performed by using Linear Discriminant Analysis, LDA. In a first step, the five selected gases are separated. As can be seen in Fig. 7.6a, discrimination of $400 \mathrm{ppm} \mathrm{H}_{2}, 400$ ppm CO, 75 ppm $\mathrm{C}_{3} \mathrm{H}_{6} 100$ ppm NO, and 25 ppm $\mathrm{NH}_{3}$ in dry nitrogen is possible. The groups are well separated in the LDA scatter plot. Hydrogen and ammonia are located further apart from the other groups, which indicates that the sensor has a higher selectivity to ammonia and hydrogen. Not only discrimination of the five gases is possible but also quantification as shown exemplarily for ammonia in Fig. 7.6b.
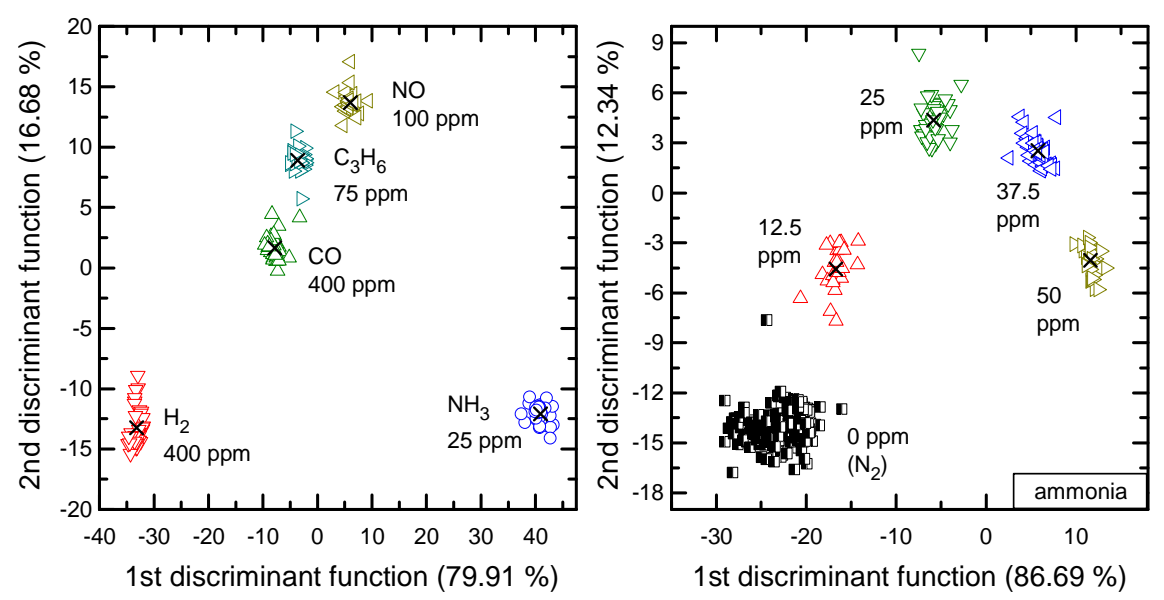

Fig. 7.6 (a) Discrimination of $\mathrm{H}_{2}, \mathrm{CO}, \mathrm{C}_{3} \mathrm{H}_{6}, \mathrm{NO}$, and $\mathrm{NH}_{3}$ in dry nitrogen, and (b) quantification of different concentrations of $\mathrm{NH}_{3}$. The $0 \mathrm{ppm}$ group, i.e., pure nitrogen, has been projected into the scatter plot. 
The LDA is first trained with $12.5,25,37.5$, and 50 ppm ammonia (open symbols). The groups are again well separated by the first discriminant function, DF1. In a next step, the obtained LDA coefficients, i.e., the training data are used for the algorithm to project unknown data points into the same scatter plot. The new points corresponding to $0 \mathrm{ppm}$, i.e., pure nitrogen (semi-solid symbols) were projected next to the lowest concentration, $12.5 \mathrm{ppm}$, used for the training. This is a test of plausibility and a good indicator for the stability of the discrimination. If the $0 \mathrm{ppm}$ group would be located somewhere else in the scatter plot, the discrimination might not be based on the gas response but rather on noise or drift. Determination of the concentration could just be done by using the value of DF1. This is also possible for the other gases as well as with oxygen in the background [283], [246].

In order to validate the suggested TCO approach, the discrimination of five gases, cf. Fig. 7.6a, where each group contains only one concentration, is considered again. The obtained LDA coefficients from this discrimination are now applied to different, unknown concentrations of propene (Fig. 7.7a semi-solid symbols). It can be observed that the projected groups representing 25, 50, and $100 \mathrm{ppm}_{3} \mathrm{H}_{6}$ are fairly close to the training group (75 ppm $\left.\mathrm{C}_{3} \mathrm{H}_{6}\right)$. A $k$-Nearest Neighbor, $\mathrm{kNN}$, classifier with $\mathrm{k}=3$ gives a correct classification rate of $82.3 \%$. Thus, the TCO approach with the selected features represents the characteristics of the gas.
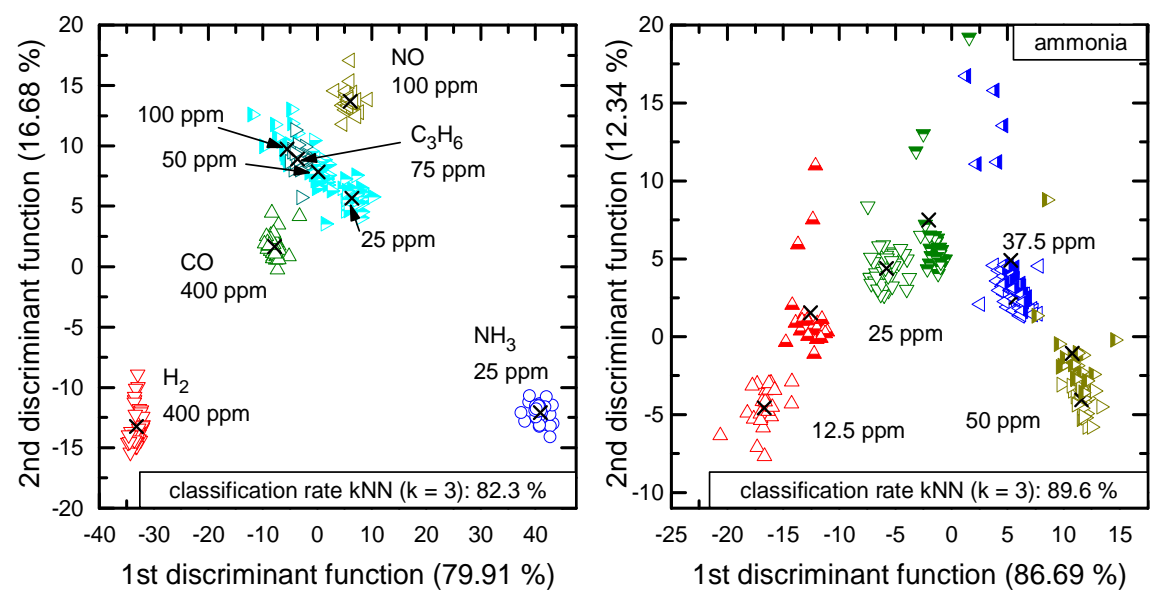

Fig. 7.7 (a) Verification of the TCO approach, and (b) investigation of the stability. Training data are marked by open symbols, projected data by semi-solid symbols. In dry nitrogen. 
Repeatability and stability of the discrimination is investigated based on the quantification of ammonia as shown in Fig. 7.6. The measurement was repeated after eight days and the new data are projected by using the LDA coefficients from the discrimination shown in Fig. 7.6. It can be observed, cf. Fig. 7.7b, that the projected groups (semi-solid symbols) are located closely to their corresponding training group (open symbol). There is a small drift visible and there are a few outliers which are probably the first cycles of each gas exposure where the sensor has not been stabilized yet. However, this is just a run-in process. A $\mathrm{kNN}$-classifier with $\mathrm{k}=3$ leads to a correct classification rate of $89.6 \%$.

Similar results for other gases and in an oxygen containing atmosphere can be found in [283], [246].

\subsubsection{Conclusion Temperature Modulation}

In this sub-chapter, the methodology of temperature cycling is systematically studied for SiC-FETs. So far, only very basic and preliminary studies can be found in literature. The presented results indicate that the temperature cycled operation, TCO, is a suitable approach for SiC-FET gas sensors to increase the selectivity. In order to highlight gas introduced changes in the dynamic sensor response, which is important to define feature intervals, the difference (normalized) signal can effectively be used. Both qualitative analyses for discrimination of individual gases as well as quantitative determination of gases have been demonstrated. Results have been verified by validation, i.e., by the projection and classification of unknown data. The stability of the method is in a reasonable range for chemical sensors. Thus, the virtual multi-sensor approach is also appropriate for field effect based gas sensors. The methodology of temperature cycled operation can now be developed further and applied to more complex scenarios. 


\subsection{Gate Bias Modulation of SiC-FETs}

In the case of three-terminal field effect transistor devices, cf. Fig. 6.7, not only the temperature can be modulated but also the applied gate bias. In this section, gate bias hysteresis is studied and the concept of gate bias cycled operation, GBCO, is introduced. This sub-chapter is based on [284], [285], [286].

\subsubsection{Design of Experiment}

It was reported that the applied gate bias has an influence on the sensing properties of a gas sensitive field effect transistor [287]. Additionally, gas significant information is contained in the transient sensor response when changing the applied bias [284]. In order to study the influence of a changing gate more systematically, it is linearly ramped from $-3 \mathrm{~V}$ to $+7 \mathrm{~V}$ and back-down again over $900 \mathrm{~s}$ each, cf. Fig. 7.8 blue curve. This symmetric pyramid-shaped gate bias cycle allows studying hysteresis effects. Schütze et al. [19] have shown that the occurrence of hysteresis in TCO is a good indicator that additional information is contained in the transient behavior of the sensors which can be used to increase the selectivity.

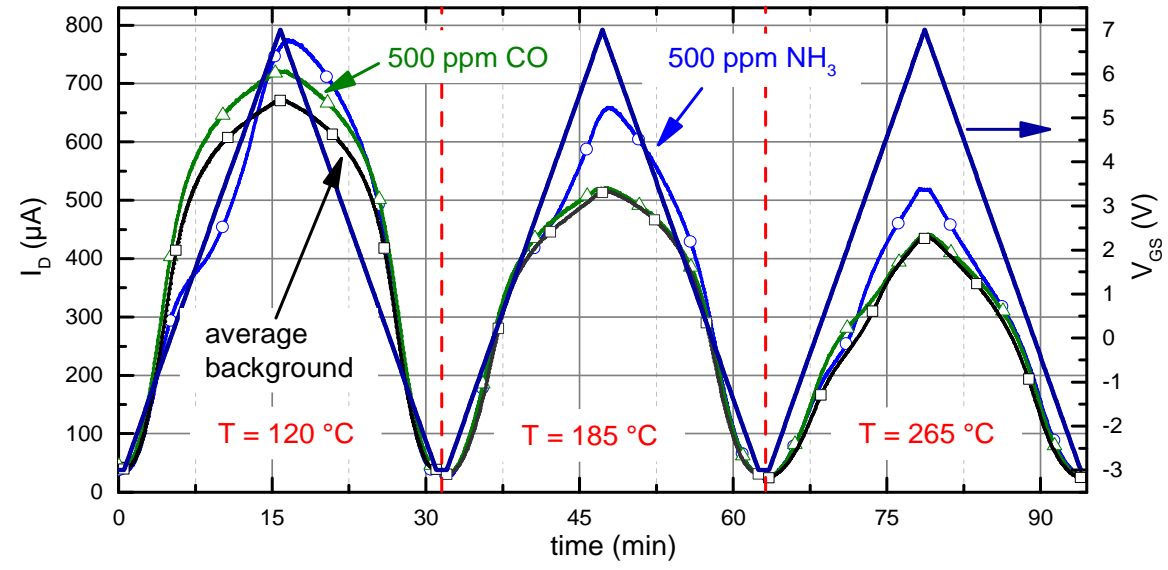

Fig. 7.8 Applied gate bias sweeps (blue) and corresponding sensor response to $500 \mathrm{ppm}$ ammonia and $500 \mathrm{ppm}$ carbon monoxide in dry synthetic air at three temperatures. Adopted from [286]. 
The mentioned gate bias cycle is repeated three times at $120^{\circ} \mathrm{C}$. Then the temperature is increased to $185^{\circ} \mathrm{C}$ the gate bias cycle is repeated three times before the temperature is increased to $265^{\circ} \mathrm{C}$. During the second bias cycle at each temperature the sensor is exposed to test gas. Fig. 7.8 shows only the second cycle at $120^{\circ} \mathrm{C}, 185^{\circ} \mathrm{C}$, and $265^{\circ} \mathrm{C}$, respectively. For each temperature the corresponding sensor response in carrier gas (dry synthetic air), 500 ppm carbon monoxide, and $500 \mathrm{ppm}$ ammonia is given. It can be observed that the sensor response for the two gases change differently with the applied bias. Already here, one can see that the sensor shows gas depended hysteresis.

\subsubsection{Hysteresis Effects}

When plotting the drain-source current over the applied gate bias, the hysteresis of the sensor becomes clearly visible as shown in Fig. 7.9a and c.

For pure nitrogen almost no hysteresis is noticeable whereas for $500 \mathrm{ppm}$ ammonia or $500 \mathrm{ppm}$ carbon monoxide the hysteresis is pronounced with a maximal height of $80 \mu \mathrm{A}$ and $140 \mu \mathrm{A}$, respectively, at a gate bias of around $+3.5 \mathrm{~V}$ and $185^{\circ} \mathrm{C}$. For higher temperature, e.g., $265^{\circ} \mathrm{C}$ the hysteresis width and height decreases. In order to highlight the gas dependency of the hysteresis curve, the difference signal $\Delta I_{D}$ was calculated as follows

$$
\Delta I_{D}=I_{D}(\text { gas })-I_{D}(\text { background })
$$

where $I_{D}$ (gas) is the hysteresis curve in a test gas atmosphere and $I_{D}$ (background) the hysteresis curve in the background, e.g., dry nitrogen. The corresponding difference hysteresis curves for $\mathrm{NH}_{3}$ and $\mathrm{CO}$ in dry nitrogen are given in Fig. 7.9b and d. Around $0 \mathrm{~V}$, a peak becomes evident which can be interpreted as a polarity dependent effect, e.g., flipping of trap states [116]. At $265{ }^{\circ} \mathrm{C}$ crossover points appear for both ammonia and carbon monoxide which indicates that at least two different and probably competing processes with different time constants are involved.

Not only temperature affects the shape of the hysteresis curve but also the level of oxygen and humidity, cf. Fig. 7.10. Background oxygen and humidity strongly 
influence the $\mathrm{CO}$ response as shown in Fig. 7.10a while it has only a minor effect on the $\mathrm{NH}_{3}$ response.
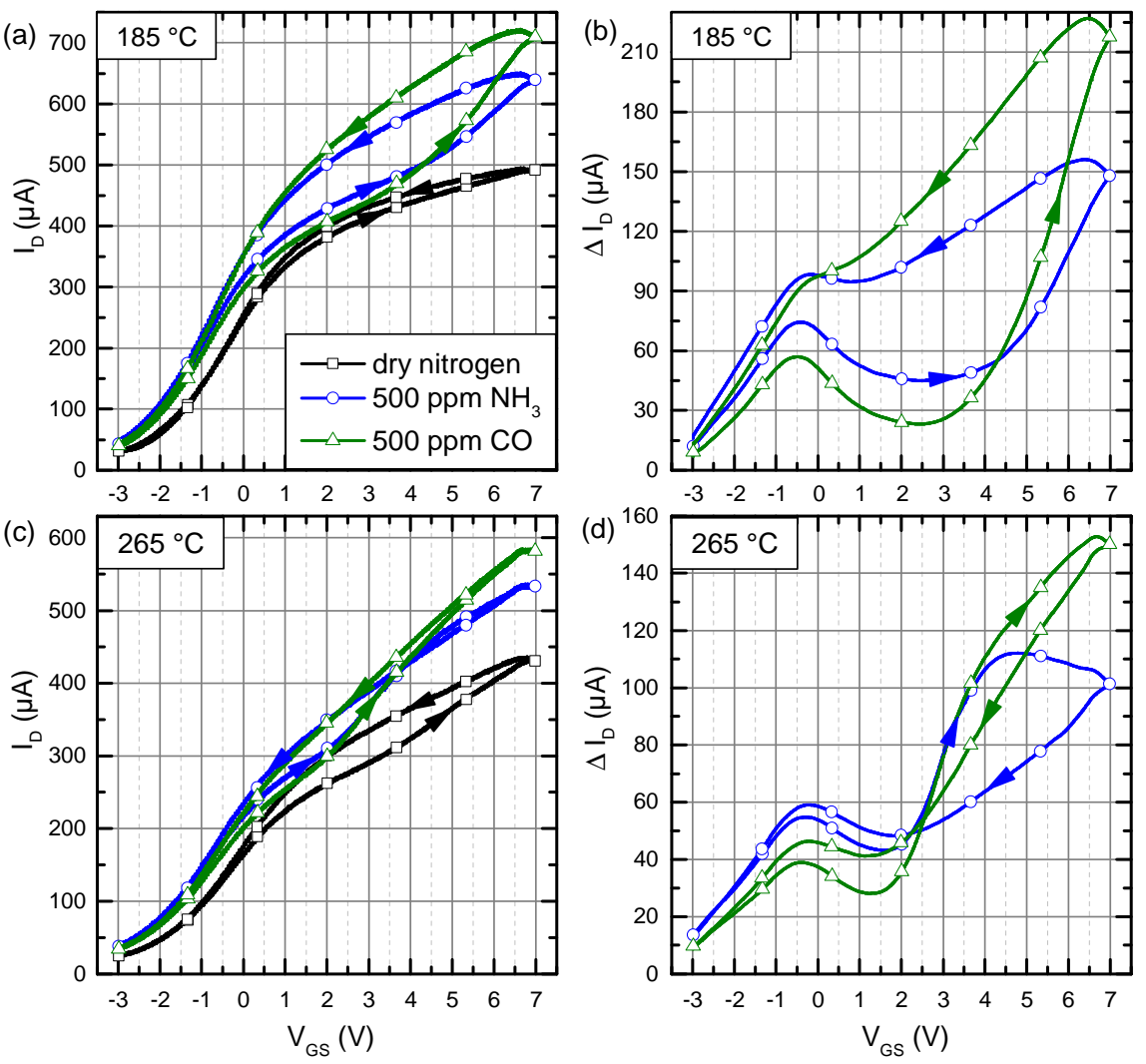

Fig. 7.9 Hysteresis curves for $\mathrm{NH}_{3}$ and $\mathrm{CO}$ in dry $\mathrm{N}_{2}$ when sweeping the gate bias from $-3 \mathrm{~V}$ to $+7 \mathrm{~V}$ in $900 \mathrm{~s}$ and back. (a) Absolute signal at $185^{\circ} \mathrm{C}$, (b) difference signal at $185^{\circ} \mathrm{C}$, (c) absolute signal at $265^{\circ} \mathrm{C}$, and (d) difference signal at $265^{\circ} \mathrm{C}$. Adopted from [286].

One reason for that could be that $\mathrm{CO}$ as a very reactive reducing gas interacts with negatively charged oxygen ions on the sensor surface. At low temperatures and sufficiently high $\mathrm{CO}$ concentration, the platinum surface of the transistor is completely covered by $\mathrm{CO}$, i.e., no adsorbed oxygen is present, whereas it is almost completely covered with adsorbed oxygen for high temperatures or a low $\mathrm{CO} / \mathrm{O}_{2}$ ratio.

In the presence of humidity, cf. Fig. 7.10b, the $\mathrm{NH}_{3}$ response especially, for negative applied gate bias changes significantly. 
Further results for instance for methane, $\mathrm{CH}_{4}$, or nitrogen monoxide, $\mathrm{NO}$, can be found elsewhere [288], [286].
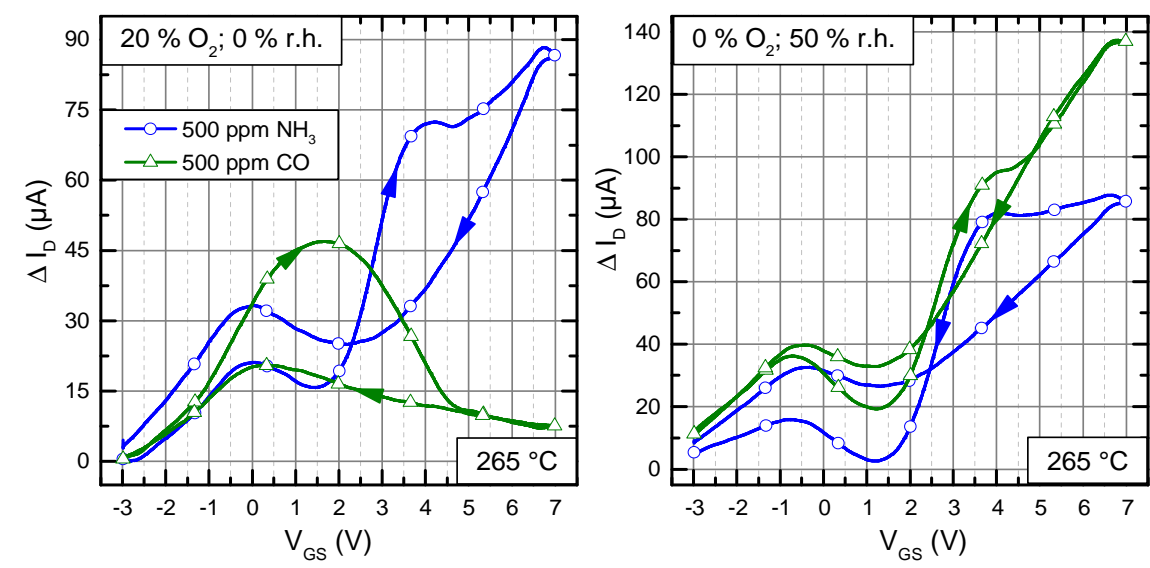

Fig. 7.10 Difference hysteresis curves for $\mathrm{NH}_{3}$ and $\mathrm{CO}$ at $265^{\circ} \mathrm{C}$ when sweeping the gate bias from $-3 \mathrm{~V}$ to $+7 \mathrm{~V}$ in $900 \mathrm{~s}$ and back again. (a) In dry synthetic air, i.e., $20 \% \mathrm{O}_{2}$ in $\mathrm{N}_{2}$, and (b) in nitrogen with $50 \%$ r.h. at $20{ }^{\circ} \mathrm{C}$. Adopted from [286].

\subsubsection{Explanations for Hysteresis}

When changing the applied gate bias continuously, gas dependent hysteresis is observed. So far, it is not completely understood what causes the hysteresis and what happens when changing the bias. However, there are a few possible explanations which will be discussed in the following.

In general, the hysteresis phenomenon is also observed for other field effect sensors, e.g., suspended gate, or nano-FET devices as presented in Section 4.3. Some of the concepts are probably also valid for SiC-FETs. Paska and Haick [116] reported that for silicon nanowire FETs the hysteresis is due to flipping of trap states and thus, a polarity dependent effect. The trap states are filled by adsorption of gas molecules on the surface of the nanowire. In the case of SiC-FETs, molecules can also directly adsorb on the insulator. As discussed in Section 3.3 different kinds of adsorption can take place whereas only chemisorption involves charge injection into the insulator, i.e., oxide, resulting in surface filled states [117], [122]. When changing the applied gate bias, these traps can partly be flipped [289]. In the description of MIS capacitor devices in Section 3.1.1, it is stated that there are basically four different kinds of 
oxide charges, cf. Fig. 3.5, interface trapped charges $Q_{i t}$, fixed oxide charges $Q_{f}$, oxide trapped charges $Q_{o t}$, and mobile ionic charges $Q_{m}$. All of them influence the flat band voltage $V_{F B}$ and thus, the threshold voltage $V_{t h}$ of the device. If due to any external effect the density of these charges is altered, the IV-curve of the sensor will change. It was reported that $Q_{f}, Q_{i t}$, and $Q_{m}$ are not affected by the gate bias [65]. Nevertheless, in the case of a sensor device, where the oxide is exposed to the environment and thus, can interact with gases, the density of some of these charges may change, in particular interface traps $Q_{i t}$ and oxide traps $Q_{o t}$. Chatty et al. reported that for SiC-FETs the hysteresis is related to changes in effective fixedoxide charge resulting from interface traps [290].

Chang et al. [203] suggested that an externally applied bias can be used to influence the adsorption and desorption rates of certain molecules. Since it is observed for SiC-FET devices that the hysteresis is strongly gas and temperature dependent, the chemical reaction on the sensor surface probably changes with the applied bias.

The SiC-FETs which are used in this study have porous platinum gates. In oxygen containing atmosphere, the surface is partly covered by oxygen ions chemisorbed to both the platinum as well as the insulator. Although, the main direction is perpendicular to the surface, an electric field can lead to spill-over of adsorbed oxygen from the platinum to the insulator and vice versa, i.e., from the insulator to the platinum (reverse spill-over). This movement of ions on the surface changes also the electric field in the underlying insulator and thus, the charge carriers in the channel of the transistor. However, since the hysteresis in air is small compared to a test gas atmosphere, this can only have a minor influence. Spill-over effects of oxygen to or from the catalyst also imply a change of active sites on the catalyst. This directly influences the response to different gases so that a change of the applied bias affects the sensing behavior of the sensor. Besides oxygen, in principle every adsorbed species on the surface can move, and if this movement leads to a change of the electric field, the IV-curve of the FET is affected. This effect is usually not much polarity depended but depends rather on the bias gradient.

Another theory involves restructuring of the sensor surface. Since the catalytic layer is highly porous and only $25 \mathrm{~nm}$ thick, an applied field can move platinum atoms on the surface. This means the structure of the catalyst changes and therewith the number of adsorption sites or the catalytic activity of those. Movement of charge, 
e.g., oxygen ions, or atoms, e.g., platinum, on the surface does usually not depend much on the polarity of the bias but rather on the bias gradient in contrast to flipping of trap states.

All of the suggested processes are slow, starting from a few seconds to even minutes which fit to the observed effects. However, deeper investigations are needed to explain the hysteresis. Especially, since also crossing-points appear in the hysteresis curves, several processes on different time scales are probably involved. For instance the hysteresis curves in dry nitrogen, cf. Fig. 7.9, show depending on the test gas at the maximum one crossing-point whereas in the hysteresis curves with $50 \%$ r.h., cf. Fig. 7.10, two crossing-points are visible. Thus, several processes which depend on the ambient conditions, are taking place when sweeping the applied gate bias.

\subsubsection{Discrimination Using Hysteresis}

The hysteresis curves given in Fig. 7.9 and Fig. 7.10 are characteristic for different gas exposures. Hence, features describing the hysteresis curve, e.g., the hysteresis width, height, or area within the curve can be extracted and used for pattern recognition. For discrimination purposes, the gate bias cycle shown in Fig. 7.11a is used.
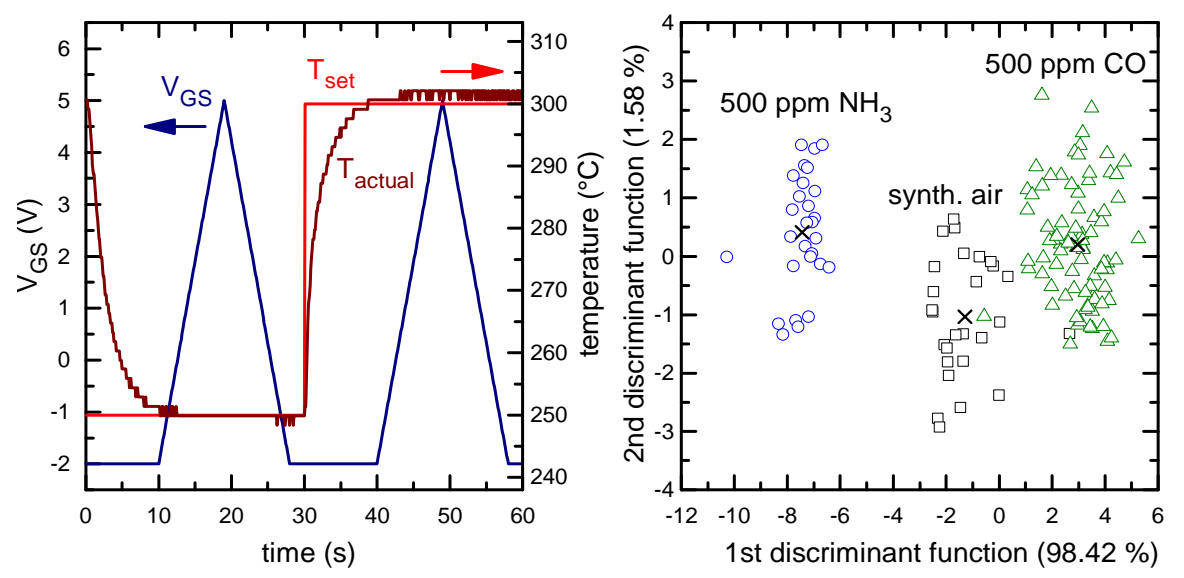

Fig. 7.11 (a) Gate bias cycle, and (b) LDA scatter plot showing the discrimination of $500 \mathrm{ppm}$ $\mathrm{NH}_{3}, 500 \mathrm{ppm} \mathrm{CO}$, and synthetic air under dry condition. Only the hysteresis height, width, and area under curve at both temperatures are used as features. 
It consists of two temperatures, i.e., $250{ }^{\circ} \mathrm{C}$ and $300{ }^{\circ} \mathrm{C}$ and on each temperature plateau the gate bias is linearly ramped from $-2 \mathrm{~V}$ to $+5 \mathrm{~V}$ in $9 \mathrm{~s}$ and back again. As gas significant features the hysteresis height, width, and area under curve at both temperatures are extracted. These six features are then used as input data for Linear Discriminant Analysis. The resulting scatter plot for the discrimination of $500 \mathrm{ppm}$ $\mathrm{NH}_{3}, 500$ ppm CO and dry synthetic air is shown in Fig. 7.11b. As can be seen the three groups are nicely separated. If only the hysteresis width, height, and area under curve from the first bias cycle are used, a similar result is obtained but the groups move closer to each other, cf. Fig. 7.12.

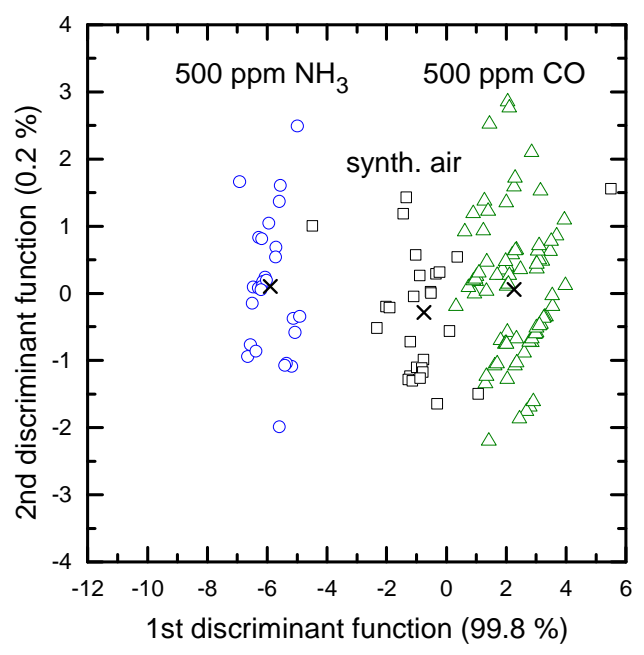

Fig. 7.12 LDA scatter plot showing the discrimination of $500 \mathrm{ppm} \mathrm{NH}_{3}, 500 \mathrm{ppm} \mathrm{CO}$, and synthetic air under dry condition. Only the hysteresis height, width, and area under curve at $260{ }^{\circ} \mathrm{C}$ are used as features.

Thus, the introduced gate bias cycled operation leads to similar results as temperature cycled operation. It should be noted that the temperature has in general a much stronger impact on the sensor response compared to the applied bias. Changing the gate bias requires a certain operating temperature and already the cycle in Fig. 7.11 uses two different temperatures. Hence, GBCO is closely connected to TCO. 


\subsubsection{Conclusion Gate Bias Modulation}

It can be concluded that modulation of the applied gate bias of a SiC-FET provides additional information regarding selectivity. The sensor exhibits gas significant hysteresis when the bias is ramped. In order to highlight the gas introduced changes, the difference signal, i.e., hysteresis curve in gas minus hysteresis curve in the background mixture, is calculated. Additionally, the hysteresis depends on the oxygen and humidity level as well as on the slope of the ramp. The occurring effects when sweeping the gate bias are not completely understood yet but possible explanations are given, involving flipping of trap states, change of the adsorption and desorption rate, spill-over and reverse spill-over effects, and restructuring of the surface. Based on the hysteresis curves features describing the curve, e.g., enclosed area or position of crossing points, are used for discrimination. Thus, the methodology of gate bias cycled operation, GBCO, is very similar to temperature cycled operation and can be seen as a new method within the virtual multi-sensor approach. These preliminary results are the basis for Section 8.3 in which temperature cycled and gate bias cycled operation is combined. 


\subsection{Advanced Signal Processing}

In this section, the methodology with respect to signal processing for dynamically operated SiC-FETs is discussed based on the general concepts already presented in Chapter 5.

\subsubsection{Feature Selection}

The main goal of pattern recognition is the reduction of dimension. This can be achieved by feature extraction, and followed by projection in a lower dimensional space, e.g., by using LDA. However, not every extracted feature is significant and contributes much to the discrimination. In order to reduce the dimension even more and to exclude non-significant features or features describing just noise or drift, feature selection can be applied. However, when using LDA for the discrimination and there is no obvious risk for over-fitting, there is no explicit need to exclude noisy features. For well-conditioned problems, these do not affect the discrimination in a negative way. There are several possibilities to select the most significant features but the different methods do not necessarily lead to the same result. Thus, feature selection depends to some extent on the experience of the user. In general, there are two different possibilities to select features: first, features can be selected before discrimination. This kind of selection can only select promising features but it cannot be determined if they are significant at this stage since features are weighted differently depending on the chosen algorithm. Secondly, features can be selected after discrimination so that only features are chosen which contribute much, i.e., which are significant.

The probably simplest and most intuitive way to exclude features that basically represent noise is to plot the feature value over the cycle number, i.e., over time. Fig. 7.13 exemplarily shows four different features and how they change over time when the concentration is increased. Features 1 and 2 represent the different concentrations well, whereas features 3 and 4 should be excluded before discrimination since they only represent noise. One possibility to automate this selection/ elimination process is to determine the correlation between the feature 
value and the concentration. If they are uncorrelated, the feature represents mainly noise and can be eliminated. However, if the number of features is small, manual selection of features is possible.

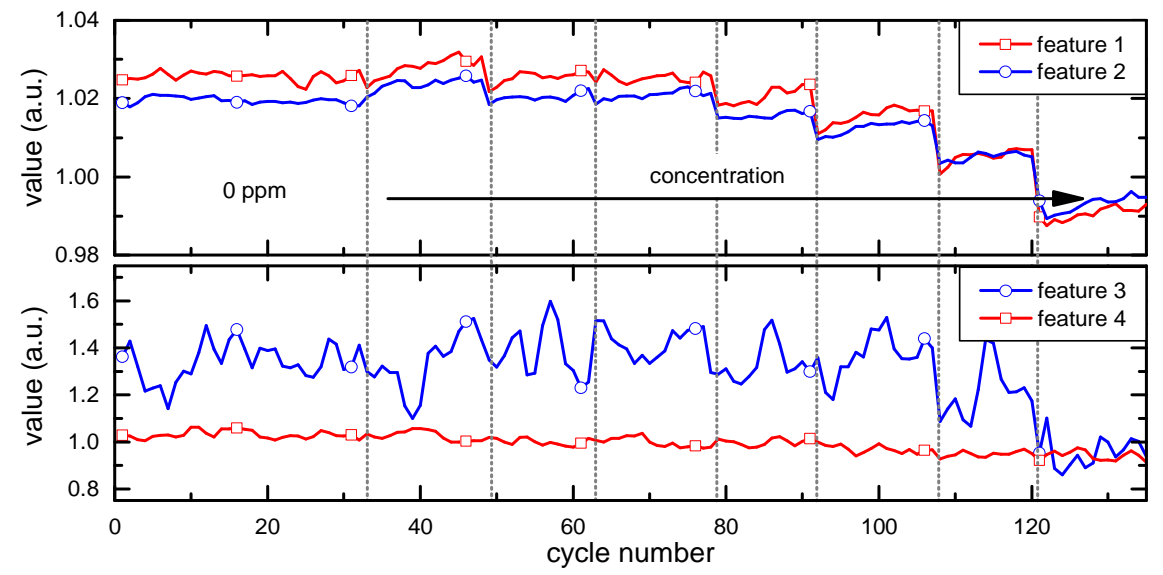

Fig. 7.13 Change of feature value over time for increasing concentration steps. (a) Promising features, and (b) features which represent only noise.

Feature selection before calculating the LDA, i.e., solving the eigenvalue problem, can be done based on statistics. The univariate F-value as defined in Eq. (5.28) or the univariate Wilks' Lambda as defined in Eq. (5.27) are suitable indicators. Both lead to the same results but the Wilks' Lambda is normalized to an interval 0..1. For promising features the F-value should be large while it should be small for the Wilks' Lambda. Fig. 7.14 shows the univariate F-value for the discrimination shown in Fig. 7.15a. There is no written rule for how many features should be selected based on the F-value.

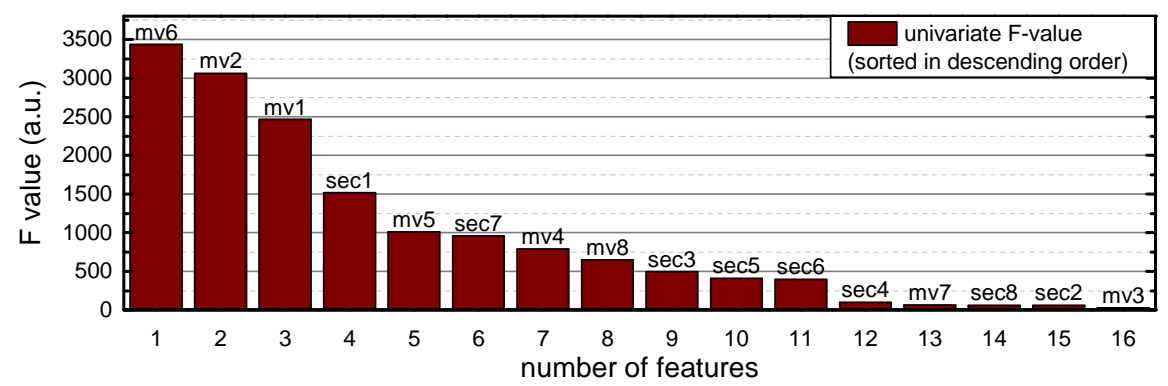

Fig. 7.14 Diagram showing the value of the univariate F-value in descending order for the discrimination shown in Fig. 7.15a. 
Another possibility to select features is by using the loadings plot. Fig. 7.15 shows the scatter and the corresponding loadings plot for the discrimination already shown in Fig. 7.6a.
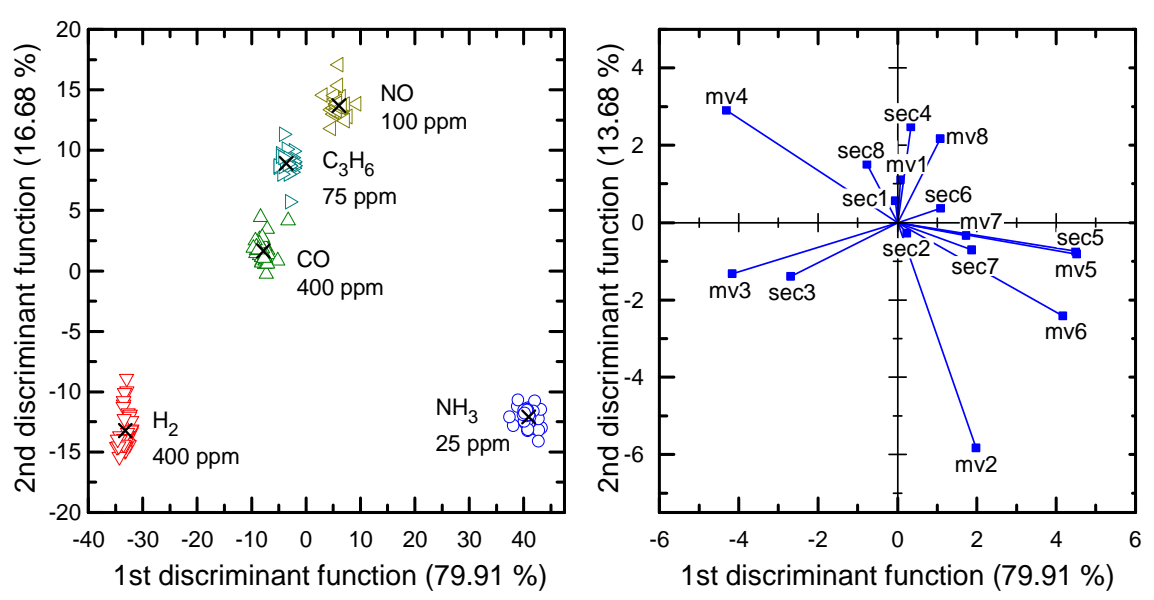

Fig. 7.15 (a) Discrimination of $\mathrm{H}_{2}, \mathrm{CO}, \mathrm{C}_{3} \mathrm{H}_{6}, \mathrm{NO}$, and $\mathrm{NH}_{3}$, and (b) corresponding loadings plot.

Using the loadings plots means the discrimination is done first and features are selected by their weight determined by the algorithm. In case of LDA, the weight of a feature is expressed in its standardized coefficient, cf. Section 5.3.3, which is independent to the scaling of different features. The loadings plot visualizes the standardized coefficients for each feature and discriminant function. Thus, features which are located close to the origin in the loadings plot, in Fig. 7.15b for instance sec1 or sec2, can be removed since they do not contribute much to the discrimination. However, there is no pre-defined threshold which can be used to select features. Another benefit of the loadings plot is that it shows how the feature contributes to the discrimination. Features pointing in the same direction, e.g., mv5 and sec5 in Fig. 7.15b, carry redundant information and it is enough to just take one of the two features. The distance of the feature loadings from the origin in the loadings plot can be expressed by the average coefficient which takes the significance of each discriminant function into account, cf. Eq. (5.26). Hence, feature selection can also be done based on the value of the average coefficient, cf. Fig. 7.16. However, this coefficient does not express redundancy of two features and should thus only be used in addition to the loadings plot. 
A more black-box approach for feature selection is the stepwise LDA, cf. Section 5.3.4. It selects the most significant features based on a combination of both the forward selection and backward elimination techniques. However, it is very difficult to interpret the made selection and it does not necessarily lead to the best discrimination. Hence, the stepwise LDA should rarely be used.

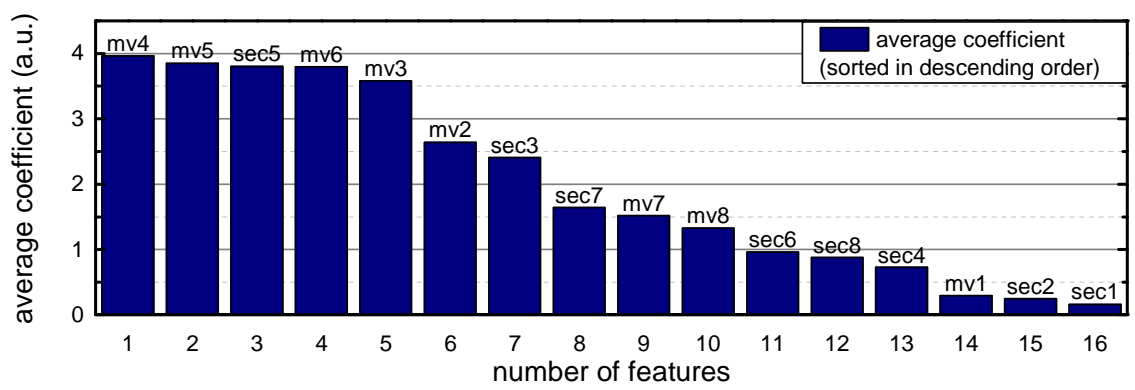

Fig. 7.16 Diagram showing the value of the average coefficient in descending order for the discrimination shown in Fig. 7.15a.

Fig. 7.17 shows the LDA scatter plots when features are selected based on the F-value, the average coefficient, and the stepwise LDA. As can be seen, there are only minor differences although, the selected features are different. Mainly the scale of the discriminant functions changes which can be used to compare the three plots regarding the quality of the discrimination. Due to that, the scales of the discriminant functions of the three scatter plots shown in Fig. 7.17 are the same.
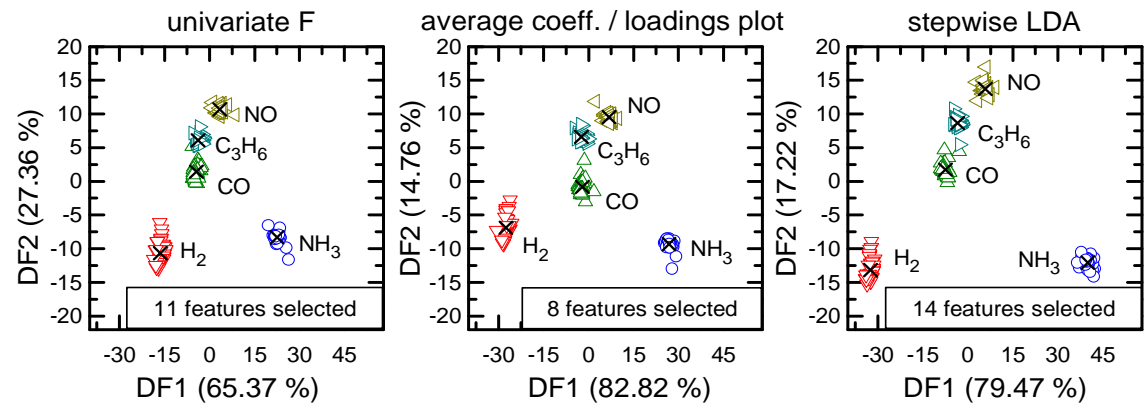

Fig. 7.17 LDA scatter plot showing the discrimination of $\mathrm{H}_{2}, \mathrm{CO}, \mathrm{C}_{3} \mathrm{H}_{6}, \mathrm{NO}$, and $\mathrm{NH}_{3}$ similar to Fig. 7.15a but after feature selection based on (a) the univariate F-value, (b) the average coefficient and the loadings plot, and (c) the stepwise LDA algorithm. 
It can be observed that the groups are located closer to each other if features are selected by their univariate F-value compared to the other two discriminations. Selection based on the univariate F-value or on the stepwise LDA is often elusive whereas selection based on the average coefficient is more intuitive. Which methods should be used, therefore depends much on the users experience and preferences. An overview is given in Table 6.

Table 6 Overview of feature selection methods and their suitability for automatization.

\begin{tabular}{|c|c|c|}
\hline method & description & suitability for automatization \\
\hline $\begin{array}{l}\text { feature value } \\
\text { over time }\end{array}$ & $\begin{array}{l}\text { - selection of possible features } \\
\text { - good for first inspection } \\
\text { - inconvenient for a large feature set }\end{array}$ & $\begin{array}{l}\text { suitable only if the correlation between } \\
\text { feature value and gas exposure is used }\end{array}$ \\
\hline $\begin{array}{l}\text { univariate } \\
\text { F-value }\end{array}$ & $\begin{array}{l}\text { - based on statistics } \\
\text { - available prior to discrimination } \\
\text { - not scaled } \\
\text { - difficult to interpret }\end{array}$ & $\begin{array}{l}\text { is used in stepwise LDA (black box) } \\
\text { leads not necessarily to the best result } \\
\text { F-value itself not suitable }\end{array}$ \\
\hline $\begin{array}{l}\text { univariate } \\
\text { Wilks' Lambda }\end{array}$ & same as univariate F-value but scaled & same as univariate F-value \\
\hline loadings plot & $\begin{array}{l}\text { - shows how features contribute to the } \\
\text { discrimination } \\
\text { - selection of significant features }\end{array}$ & $\begin{array}{l}\text { suitable in combination with average } \\
\text { coefficient }\end{array}$ \\
\hline $\begin{array}{l}\text { standardized } \\
\text { LDA coefficient }\end{array}$ & $\begin{array}{l}\text { - selection of significant features } \\
\text { - coefficients for every discriminant } \\
\text { function }\end{array}$ & $\begin{array}{l}\text { not suitable since coefficient for each } \\
\text { discriminant function and not entire } \\
\text { discrimination }\end{array}$ \\
\hline $\begin{array}{l}\text { average } \\
\text { coefficient }\end{array}$ & $\begin{array}{l}\text { - selection of significant features } \\
\text { - good for interpretation (in } \\
\text { combination with loadings plot) }\end{array}$ & $\begin{array}{l}\text { suitable but does not exclude } \\
\text { redundant features. Thus, combination } \\
\text { with loadings plot }\end{array}$ \\
\hline
\end{tabular}

If not only the reduction of dimension is important but also how a features contribute to the discrimination, the most convenient methods for feature selection is a combination of the average coefficient together with the loadings plot.

In order to compare different scatter plots with each other, there are several parameters which try to give a measure of the quality of the discrimination: The discriminant criterion itself, cf. Eq. (5.18), or the multivariate Wilks' Lambda, cf. Eq. (5.24), are statistical parameters that can be used. However, their calculation 
is based on the within and between groups sums of squares matrixes, i.e., $\boldsymbol{W}$ and $\boldsymbol{B}$ respectively. Therefore, they just provide an indication for the quality of the discrimination based on information which is available before solving the eigenvalue problem, i.e., calculating the LDA. They also strongly depend on the number of groups, features, and how well the problem is conditioned. The absolute value of those parameters is not meaningful and hard to interpret. Nevertheless, they can be used to compare different scatter plots of the same problem relatively to each other. In literature, the cluster separability $J$, cf. Eq. (5.23), is often used to describe the quality of the discrimination. This value is also based on the two sums of squares matrixes but the interpretation is easier since it is scaled. Nevertheless, for instance, overlapping of two groups is often not well expressed by this value. Another possibility to estimate the quality of an LDA plot is using cross-validation and based on this, the correct classification rate, cf. Section 5.5. Overlapping groups in the scatter plot would lead to misclassification and thus, lower the correct classification rate. In conclusion, the most meaningful parameter to describe the quality of discrimination is the cross-validation rate. Besides the quality, it provides also information about the stability of the discrimination (with respect to over-fitting) and the individual cross-validation rates indicate problematic groups due to overlapping. However, if two LDAs lead to perfect discrimination, the cross-validation rate does not provide any information which of the two is better in terms of distance between neighboring groups which might be of interest for optimization processes.

\subsubsection{The Importance of the Training}

Stability of the built model, i.e., the discrimination, is always a crucial point. First of all, for building the model, i.e., for the training, as many observations as possible should be used due to the fact that LDA is an algorithm based on statistics. Typically, each gas exposure in the training should contain at least 15 to 20 cycles, i.e., observations, to achieve a stable discrimination. For the LDA it is also important that the different groups to be discriminated are of approximately the same size.

If, for instance, several different gases should be separated, it is usually not sufficient to train the model with just one concentration per gas. In that case, the model only knows these single concentrations but in reality it is highly unlikely that exactly this concentration of a particular gas is exposed to the sensor. Under lab conditions and 
for only small variations of the concentration, the model might be stable but in general it is not. In order to increase the robustness of the discrimination, it is good practice to include as much information as possible. For the discrimination of different gases, several concentrations of each gas should be included in the training. Quite often it is sufficient to use a high and a low concentration in order to make the model stable against variations in concentration. The same strategy can be applied to suppress the influence of humidity. By including at least two different levels of humidity in the training, the built model becomes less sensitive to changes in humidity. In the same way, the influence of sensor drift can be reduced when it is included in the training [291], cf. Section 8.3.6. Theoretically, it should also be possible to combine data from several sensors in the training in order to compensate for variations between different sensors.

The quality of the discrimination itself is usually reduced when including different concentrations or levels of humidity in the training but the stability and the ability to classify unknown data correctly is strongly increased. Nevertheless, it is not possible to include everything in the training since the quality of the discrimination decreases and at some point the different groups start to overlap. Self-evidently, if the crossvalidation rate of the discrimination, i.e., the training, is low due to overlapping of the groups, the classification of unknown data will be even worse. In that case, it is not possible to do the discrimination at once but rather in two or more steps [292], [293], [294]. This is known as hierarchical discrimination which is discussed in the next section.

\subsubsection{Hierarchical Discrimination}

For complex problems, it is often impossible to build a discrimination model which is robust against, e.g., any variations in the background. A typical example is the quantification of a specific gas in a background of other interfering gases or when the level of humidity is changing. In order to solve this issue, a hierarchical strategy can be used as shown in Fig. 7.18. The main idea behind is a decision tree. In the shown example, in a first step the background is determined by a classifier. Based on its decision a new set of test data for this background is used to determine the concentration of the test gas. 


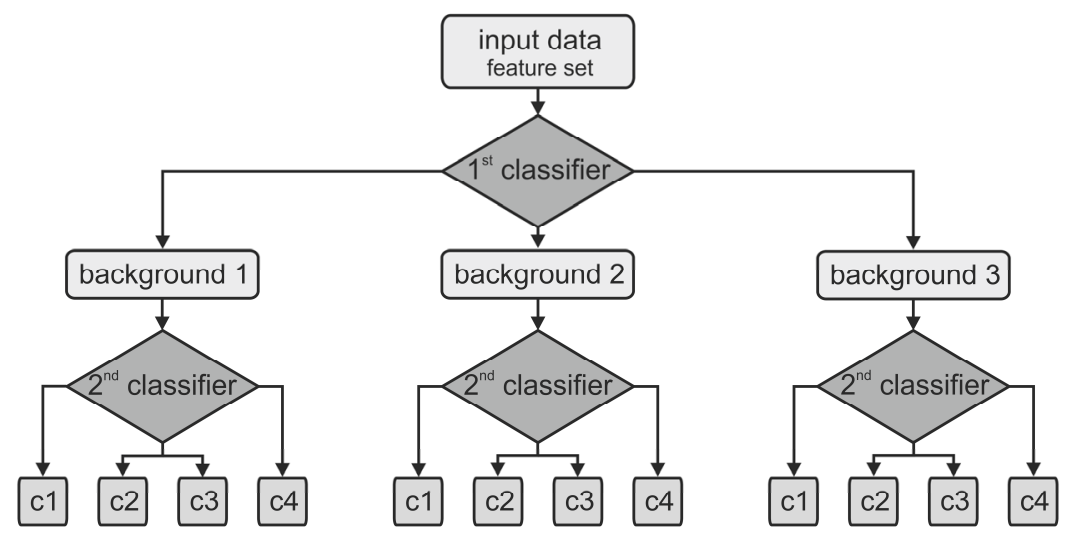

Fig. 7.18 Strategy of hierarchical discrimination.

The number of layers used in the hierarchical strategy depends on the complexity of the given problem. However, the amount of training data needed increases considerably for each added layer. Thus, not more than three layers are practically used. Also the stability of the entire decision process decreases with every layer. Therefore, it is also important to include as much information in every layer as possible to keep the number of layers small. The same principle applies for the granularity in each layer, e.g., the number of background mixtures needed to be discriminated in the first layer, cf. Fig. 7.18. With every additional class per layer, the complexity increased considerably. Hence, as much information as possible should be included in each class. De facto, there is always a tradeoff between complexity, stability, and accuracy.

If the first layer is, for instance, to distinguish between different levels of humidity, e.g., $25 \%, 50 \%, 75 \%$ r.h., the hierarchical strategy shown in Fig. 7.18 should be changed as shown in Fig. 7.19. The first layer has only two cases but each case consists of two different levels of humidity. It should be noted that the intermediate level of humidity is contained in both cases. These two groups, i.e., cases, would probably overlap in a 1D-LDA scatter plot. However, the first layer should just determine if the humidity level is rather high or low. Since there are two different levels combined in each case, the quantification done in layer 2 is nearly independent of humidity. 


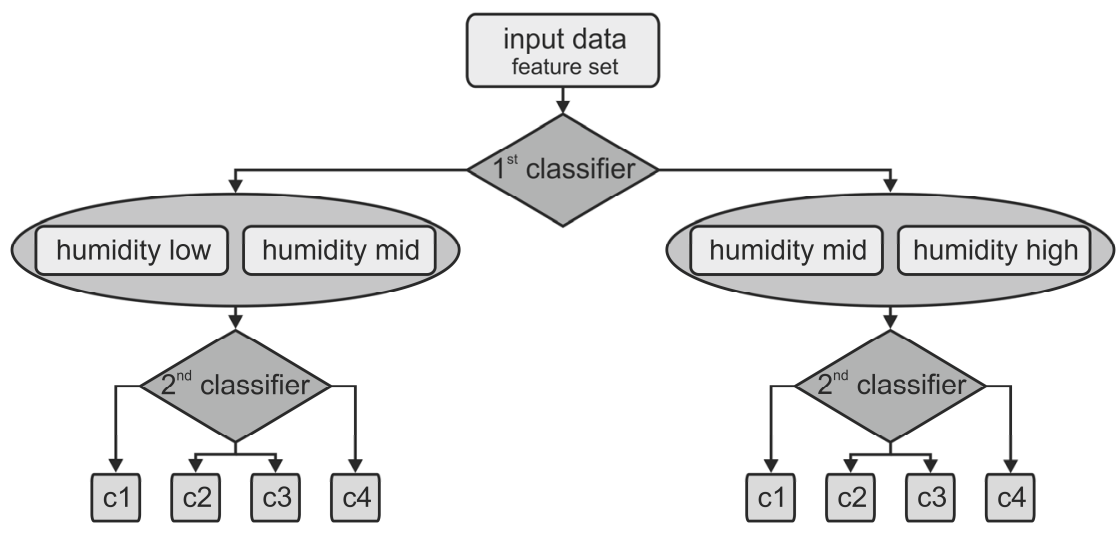

Fig. 7.19 Modified hierarchical strategy for improved stability.

\subsubsection{Conclusion Advanced Signal Processing}

Several methods for feature selection have been presented and discussed. Basically, features can be selected before, i.e., selection of promising features, and after the discrimination, i.e., selection of significant features. For feature pre-selection, visualization of the feature value over time is the most suitable method. Automatization can be implemented based on the correlation between the feature value and the gas exposure [247]. Selection of significant features after discrimination should be done based on the loadings plot which provides a good visualization of how each feature contributes to the discrimination.

The quality of the discrimination is best expressed by the cross-validation rate. The individual validation rates together with the confusion matrix provide information about, e.g., overlapping groups. The commonly used statistical parameters like the multivariate Wilks' Lambda or the cluster separability are based on parameters which are available prior to discrimination. Interpretation is often difficult but these parameters are useful to compare to discriminations relatively to each other.

In order to make the discrimination robust, as much information as possible should be contained in the training phase. For instance, if variations in the background mixture are expectable, the training data set should contain at least the lowest and highest concentration of the background. However, if these variations have a strong impact on the sensor response and the discrimination process, a hierarchical strategy might be conceivable. 



\section{Results and Discussion}

The chapter is divided into three main parts, in which the earlier suggested methodology is applied to more specific scenarios. First, the suitability of silicon carbide field effect transistors, SiC-FETs, run in temperature cycled operation, TCO, is studied for $\mathrm{NO}_{\mathrm{x}}$ detection in a high background of other exhaust gases. After that, the sensors, again run in TCO, are applied to the field of indoor air quality measurement where volatile organic compounds, VOCs, need to be detected in the low ppb-range. The last section of this chapter deals with the combination of temperature and gate bias cycled operation, GBCO.

\subsection{Exhaust Monitoring $-\mathrm{NO}_{\mathrm{x}}$ Detection}

Based on the results of preliminary studies presented in Section 7.2, temperature cycled operation of SiC-FETs is used for exhaust monitoring. In the first section, the design of experiment with the chosen gas profile is presented. Then, a methodology of finding a suitable temperature cycle for $\mathrm{NO}_{\mathrm{x}}$ detection in a high background of other exhaust gases is suggested in Section 8.1.2. The dynamic and quasi-static sensor responses as well as the signal pre-processing and the feature extraction are given in Section 8.1.3. In the following section, results regarding the quantification of $\mathrm{NO}_{\mathrm{x}}$ in a high background using Linear Discriminant Analysis and Partial Least Squares Regression are presented. Section 8.1.5 deals with the compensation of a changing background using a hierarchical strategy. This subchapter is mainly based on [295], [294], [296]. 


\subsubsection{Design of Experiment}

In this study, two-terminal enhancement type SiC-FET sensors as described in Section 6.2.2 are used. In the following, only results of a platinum gate SiC-FET are shown while results of an iridium SiC-FET can be found in [296]. The sensors were operated in a constant current mode, i.e., $I_{D}=$ const. $=100 \mu \mathrm{A}$.

As target gases, nitrogen monoxide, $\mathrm{NO}$, and nitrogen dioxide, $\mathrm{NO}_{2}$, are chosen. $\mathrm{A}$ test profile is defined with a constant ratio ${ }^{31}$ between $\mathrm{NO}$ and $\mathrm{NO}_{2}$ of $10: 1$, in the following called $\mathrm{NO}_{\mathrm{x}}$, covering a concentration range from $50 \mathrm{ppm}$ up to $200 \mathrm{ppm}$ $\mathrm{NO}$ in $25 \mathrm{ppm}$ steps, corresponding to $5 \mathrm{ppm}$ up to $20 \mathrm{ppm} \mathrm{NO}_{2}$. As interfering gases, typical exhaust components like carbon monoxide, $\mathrm{CO}$, ethene, $\mathrm{C}_{2} \mathrm{H}_{4}$, as representatives for hydro carbons, as well as ammonia, $\mathrm{NH}_{3}$, which is used in selective catalytic reduction, $\mathrm{SCR}$, systems, are chosen.

A standard background mixture consisting of typical exhaust gases, i.e., $600 \mathrm{ppm}$ $\mathrm{CO}, 50 \mathrm{ppm} \mathrm{C}_{2} \mathrm{H}_{4}$, and $60 \mathrm{ppm} \mathrm{NH}_{3}$ is defined; cf. Fig. 8.1 label "standard". The carrier gas is $10 \%$ oxygen in nitrogen with $50 \%$ relative humidity, r.h., at $20{ }^{\circ} \mathrm{C}$. At the beginning of each $\mathrm{NO}_{\mathrm{x}}$-profile, a short $\mathrm{NO}_{\mathrm{x}}$ exposure is used as an initial peak in order to have a well-defined starting situation, cf. Fig. 8.1. However, this initial exposure is not used for evaluation. The standard test profile as described above is used for defining a suitable temperature cycle in Section 8.1.2 as well as for the results presented in Sections 8.1.3 and 8.1.4 if not specified otherwise.

However, in real application the background may change. In order to simulate a changing background, the standard test profile is extended, cf. Fig. 8.1. For each of the three background gases, i.e., $\mathrm{CO}, \mathrm{C}_{2} \mathrm{H}_{4}$, and $\mathrm{NH}_{3}$, four concentrations different from the standard mixture are used, cf. Table 7. In each modified background three $\mathrm{NO}_{\mathrm{x}}$, i.e., $\mathrm{NO} / \mathrm{NO}_{2}$ concentrations 50/5 ppm, 125/12.5 ppm, and 200/20 ppm are applied to the sensor as shown in Fig. 8.1. This extended gas profile is used for investigating the influence of a changing background as discussed in Section 8.1.5.

\footnotetext{
${ }^{31}$ In real applications, the ratio is usually not constant and varies a bit with a typical $\mathrm{NO}_{2} / \mathrm{NO}_{\mathrm{x}}$ ratio in diesel exhaust between $6 \%$ and $9 \%$ [30], [300]. However, in order to develop a methodology it is assumed that the ratio $\mathrm{NO} / \mathrm{NO}_{2}$ is constant of $10: 1$ in order to limit the complexity.
} 


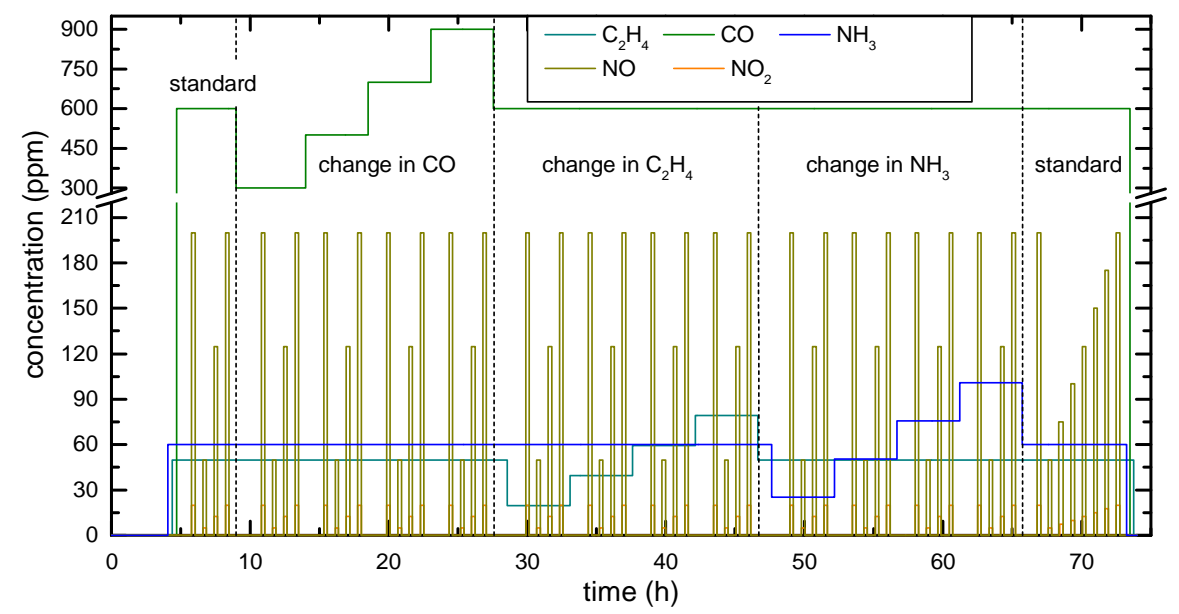

Fig. 8.1 Test gas profile. The ratio between $\mathrm{NO}$ and $\mathrm{NO}_{2}$ is kept constant at 10:1. The concentration range of $\mathrm{NO} / \mathrm{NO}_{2}$ starts at $50 / 5 \mathrm{ppm}$, and increases in 25/2.5 ppm steps up to $200 / 20 \mathrm{ppm}$. The standard mixture consists of $600 \mathrm{ppm} \mathrm{CO}, 50 \mathrm{ppm} \mathrm{C}_{2} \mathrm{H}_{4}$, and $60 \mathrm{ppm} \mathrm{NH}_{3}$ in an atmosphere of $10 \%$ oxygen in nitrogen.

In the extended profile, it is assumed that the concentration of only one of the background gases will change at a time. This is a simplification in order to develop a methodology without expanding the gas profile and measurement time extensively. Also, the ratio between $\mathrm{NO}$ and $\mathrm{NO}_{2}$ is kept constant which might not be the case in real application. The influence of changing humidity above $10 \%$ r.h., and an oxygen level above $5 \%$ is rather small [98], [138]. Again, the aim of this study is to develop a methodology for exhaust monitoring rather than optimization.

Table $7 \quad$ Overview of different background mixtures in the extended gas profile.

\begin{tabular}{lccc}
\hline $\begin{array}{l}\text { background } \\
\text { mixture }\end{array}$ & $\begin{array}{c}\text { carbon monoxide } \\
(\mathbf{p p m})\end{array}$ & $\begin{array}{c}\text { ethene } \\
(\mathbf{p p m})\end{array}$ & $\begin{array}{c}\text { ammonia } \\
(\mathbf{p p m})\end{array}$ \\
\hline standard & 600 & 50 & 60 \\
change in $\mathbf{C O}$ & $300,500,700,900$ & 50 & 60 \\
change in $\mathbf{C}_{2} \mathbf{H}_{4}$ & 600 & $20,40,60,80$ & 60 \\
change in $\mathbf{N H}_{3}$ & 600 & 50 & $25,50,75,100$ \\
\hline
\end{tabular}




\subsubsection{Temperature Cycle for $\mathrm{NO}_{x}$ Detection}

One possibility to tailor a cycle to a specific application is to use points of maximum sensitivity / response towards the test gases as plateaus in the cycle. In order to find a suitable temperature cycle, the sensor response towards $100 \mathrm{ppm}$ NO and $10 \mathrm{ppm}$ $\mathrm{NO}_{2}$ in the standard mixture of other exhaust gases as defined in Section 8.1.1 is studied as a function of the operating temperature. For this, the temperature is ramped continuously from $100^{\circ} \mathrm{C}$ up to $400^{\circ} \mathrm{C}$ in $45 \mathrm{~min}$, i.e., $6.7^{\circ} \mathrm{C} / \mathrm{min}$, cf. Fig. 8.2. In order to highlight points of maximum sensor response, the difference sensor signal $\Delta V_{D S}=V_{D S_{\text {mixture }}}-V_{D S_{N O / N O_{2}}}$ for $\mathrm{NO}$ and $\mathrm{NO}_{2}$ is shown in Fig. 8.2. Based on the difference signal, temperatures of high sensor response are extracted.

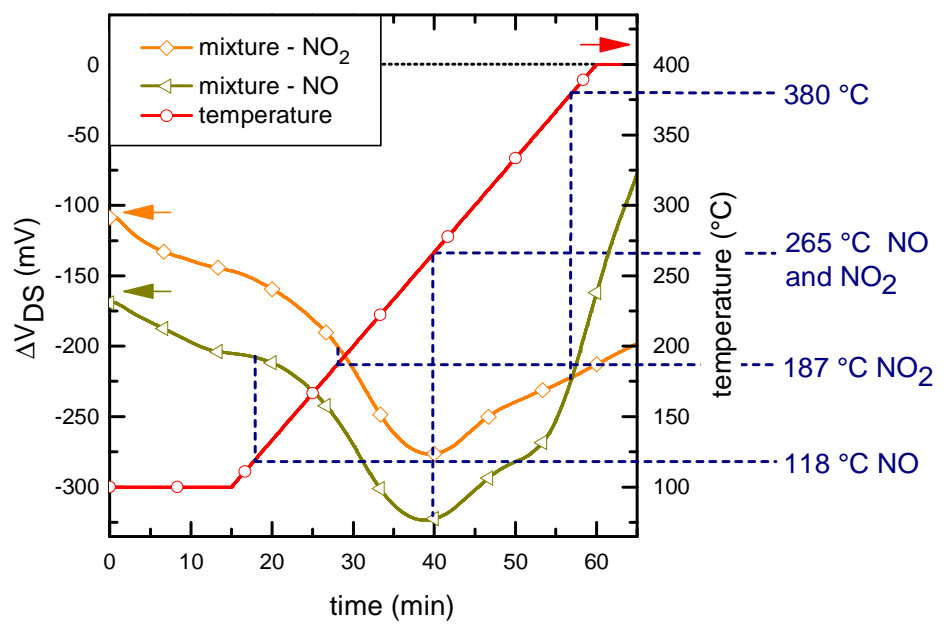

Fig. 8.2 Difference in the sensor response between a mixture of $600 \mathrm{ppm} \mathrm{CO}, 50 \mathrm{ppm} \mathrm{C}_{2} \mathrm{H}_{4}$, and $60 \mathrm{ppm} \mathrm{NH}_{3}$, and either $100 \mathrm{ppm} \mathrm{NO}$ (yellow triangles) or $10 \mathrm{ppm} \mathrm{NO}_{2}$ (orange diamonds) in a humid atmosphere, i.e., $50 \%$ r.h., of $10 \%$ oxygen in nitrogen. The temperature is increased linearly from $100{ }^{\circ} \mathrm{C}$ up to $400{ }^{\circ} \mathrm{C}$ in $45 \mathrm{~min}$ (red circles). Adopted from [273].

At $118{ }^{\circ} \mathrm{C}$ the difference signal for $\mathrm{NO}$ remains almost constant for a small temperature range, and shows then an inflection point which is an indication that this temperature possibly represents discriminatory information. A temperature of $187^{\circ} \mathrm{C}$ is also identified since the difference signal for $\mathrm{NO}_{2}$ has a large gradient. The maximal sensor response for $\mathrm{NO}$ and $\mathrm{NO}_{2}$ is obtained at $265^{\circ} \mathrm{C}$. For temperature 
cycled operation it is often beneficial to include a high temperature phase in the cycle in order to "clean" the sensor surface. At high temperatures, the desorption rate of adsorbed species, which just block adsorption sites on the sensor surface, is higher. Although, silicon carbide is stable up to $1,000^{\circ} \mathrm{C}$, the biggest issues for making high temperature devices are the ohmic contacts, and above $400{ }^{\circ} \mathrm{C}$ the porous platinum on the gate of the FET starts restructuring. Thus, a cleaning temperature of $380{ }^{\circ} \mathrm{C}$ is chosen, compromising a sufficiently high temperature, and a temperature which does not change the morphology of the sensing layer. Additionally, the sensor response at $380{ }^{\circ} \mathrm{C}$ is still rather high so that data from this part of the temperature cycle can be used for discrimination purposes, also. In fact, the response curves for $\mathrm{NO}$ and $\mathrm{NO}_{2}$ are crossing at $380{ }^{\circ} \mathrm{C}$ with a decreasing response for NO, cf. Fig. 8.2, which indicates that the sensitivity towards $\mathrm{NO}$ at this temperature is lower than for $\mathrm{NO}_{2}$.

Based on this, the temperature cycle shown in Fig. 8.3 is designed consisting of four temperature plateaus. The total duration of the temperature cycle is $45 \mathrm{~s}$.

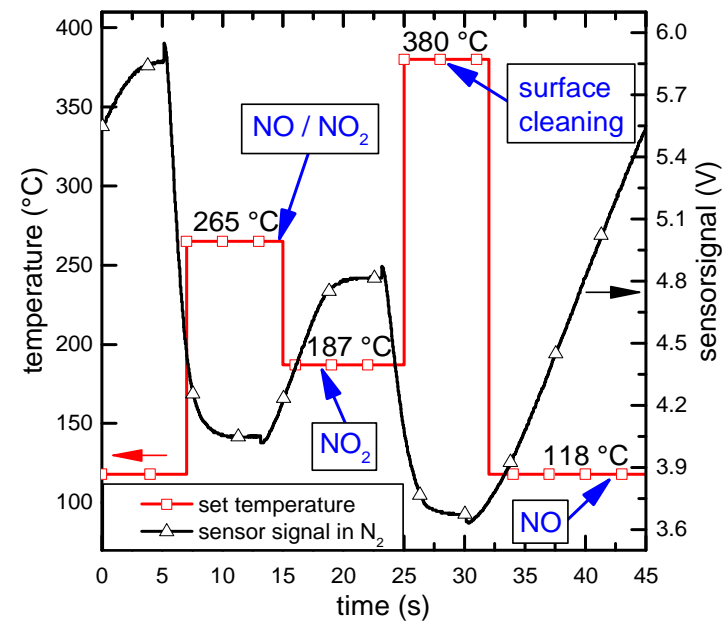

Fig. 8.3 Designed temperature cycle for $\mathrm{NO}_{\mathrm{x}}$ detection (red squares) and sensor signal in pure nitrogen (black triangles). Adopted from [273].

\subsubsection{Sensor Response and Signal Processing}

After designing a temperature cycle, the sensor response towards all chosen test gases, i.e., 25, 50, and 75 ppm $\mathrm{C}_{2} \mathrm{H}_{4}, 300,600$, and $900 \mathrm{ppm} \mathrm{CO}, 5$, 10, 15, and $20 \mathrm{ppm} \mathrm{NO} 2,50,100,150$, and 200 ppm NO, and 30, 60, and $90 \mathrm{ppm} \mathrm{NH}_{3}$, in $40 \%$ 
humid nitrogen with $10 \%$ oxygen is studied. As can be observed in Fig. 8.4, the sensor responds mainly to $\mathrm{NO}$ and $\mathrm{NO}_{2}$ at the chosen temperatures.

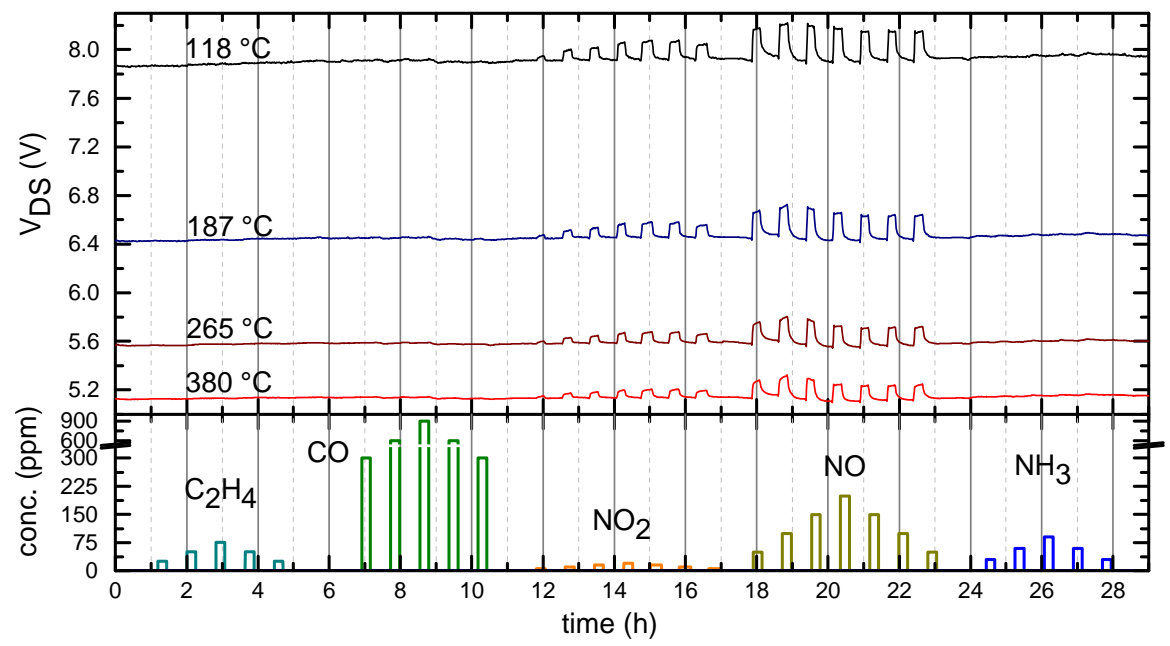

Fig. 8.4 Quasi-static sensor response towards $\mathrm{C}_{2} \mathrm{H}_{4}, \mathrm{CO}, \mathrm{NO}_{2}, \mathrm{NO}$, and $\mathrm{NH}_{3}$ in a humid atmosphere, i.e., $50 \%$ r.h., with $10 \%$ oxygen in nitrogen. Adopted from [273].
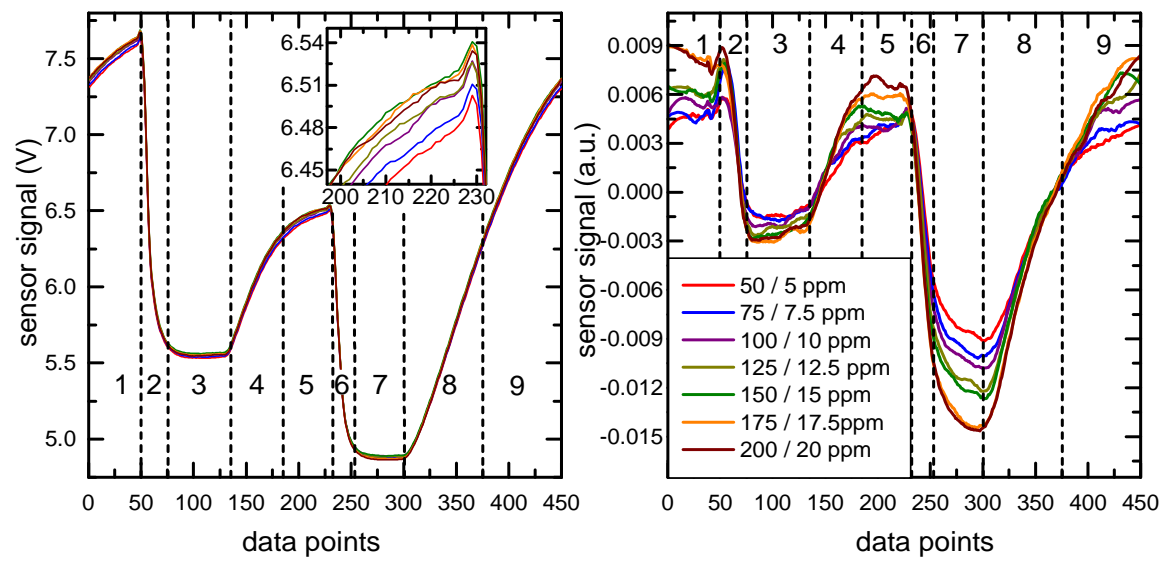

Fig. 8.5 Dynamic sensor response of different $\mathrm{NO} / \mathrm{NO}_{2}$ concentrations in a mixture of $600 \mathrm{ppm}$ $\mathrm{CO}, 50$ ppm $\mathrm{C}_{2} \mathrm{H}_{4}$, and 60 ppm $\mathrm{NH}_{3}$ with $50 \%$ r.h. and $10 \% \mathrm{O}_{2}$. (a) Raw signal with an insert showing a detailed view of the response at $187^{\circ} \mathrm{C}$, and (b) difference (normalized) signal. Normalization is done by setting the cycle mean to 1 . Nine intervals are marked for feature extraction. 
However, the change in sensor response due to temperature is much higher compared to the change due to different gas concentrations, cf. Fig. 8.5a. In the dynamic sensor response shown in Fig. 8.5a almost no changes in the sensor signal for the different $\mathrm{NO}_{\mathrm{x}}$ concentrations are visible.

Therefore, the difference signal should be used for defining the feature intervals. The normalized difference signal is shown in Fig. 8.5b. Normalization is done by setting the cycle mean to one as it is described in Section 7.2. The cycle is divided into nine intervals for the feature extraction as shown in Fig. 8.5. In each interval the mean value and secant, i.e., slope of the sensor signal are extracted as gas specific features.

\subsubsection{Quantification of $\mathrm{NO}_{x}$}

The extracted features are used for discrimination of seven different $\mathrm{NO}_{\mathrm{x}}$ concentrations in the standard background using first Linear Discriminant Analysis. Each $\mathrm{NO}_{\mathrm{x}}$ group contains two exposures of the corresponding concentration. In Fig. 8.6a the resulting LDA scatter plot is shown. Separation of the different concentrations is possible. Only the higher concentrations of $175 / 17.5 \mathrm{ppm}$ and 200/20 ppm $\mathrm{NO}_{x}$, overlap to some extent. At these high concentrations the sensor is probably already saturated which makes a separation difficult.
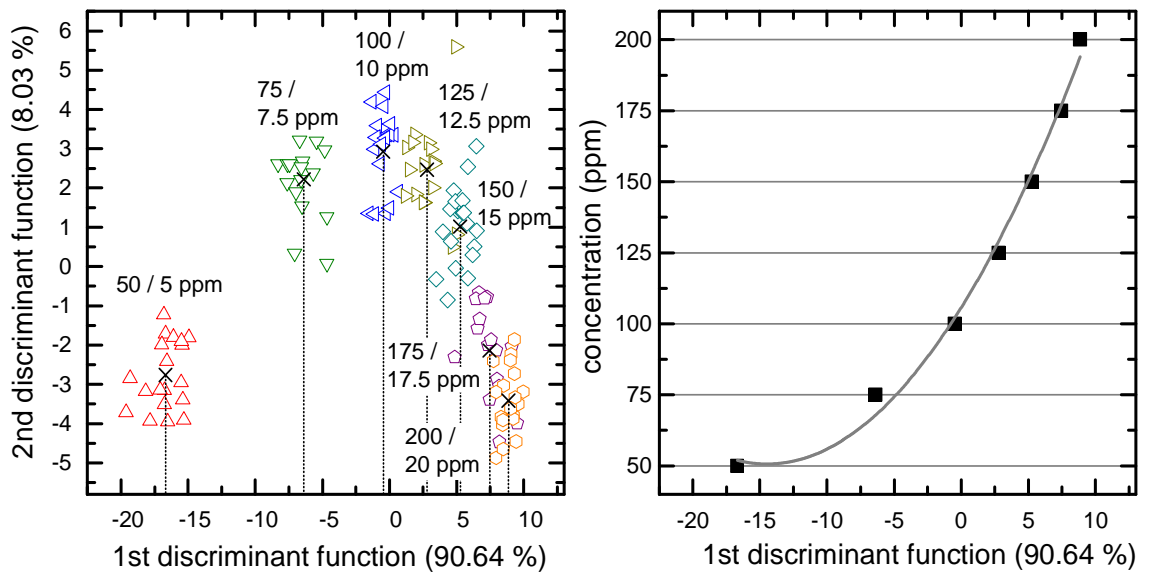

Fig. 8.6 (a) LDA scatter plot showing the discrimination of seven $\mathrm{NO}_{\mathrm{x}}$ concentrations in the standard mixture at $60 \%$ r.h., and (b) second order polynomial fit of the group centroids over their value of DF1. 
Leave-one-out cross-validation, LOOC, with a $\mathrm{kNN}$-classifier, $\mathrm{k}=5$, is used to test the result and to estimate the quality of the discrimination. The overall correct validation rate is almost $89 \%$, cf. Table 8 . In general, the validation rate for the single concentrations is high, except for $175 / 17.5 \mathrm{ppm} \mathrm{NO}$.

Table 8

Leave-one-out cross-validation using $\mathrm{kNN}$-classifier with $\mathrm{k}=5$.

\begin{tabular}{|c|c|c|c|}
\hline group & $\begin{array}{r}\text { classification } \\
\text { rate }\end{array}$ & group & $\begin{array}{r}\text { classification } \\
\text { rate }\end{array}$ \\
\hline overall & $88.8 \%$ & $125 / 12.5 \mathrm{ppm} \mathrm{NO} \mathrm{N}_{\mathrm{x}}$ & $76.5 \%$ \\
\hline $50 / 5$ ppm NO & $100 \%$ & $150 / 15$ ppm NO & $100 \%$ \\
\hline $75 / 7.5$ ppm NOx & $100 \%$ & $175 / 17.5$ ppm NO & $56.3 \%$ \\
\hline $100 / 10$ ppm NO & $94.4 \%$ & $200 / 20$ ppm NOx & $89.5 \%$ \\
\hline
\end{tabular}

Discrimination of the different concentration groups is mainly, i.e., to $90.6 \%$, done by the first discriminant function, DF1. The second discriminant function, DF2, represents only $8 \%$ of the discriminatory information, and represents mainly drift in the given example. An indication for drift is the "banana-shape" of the groups in the LDA scatter plot.

For determination of the concentration, the group centroids of each concentration can be plotted and fitted by a second order polynomial as a function of their DF1 value, cf. Fig. 8.6b. If data points of an unknown concentration are projected based on the discriminant coefficients obtained from the training, only their DF1 value together with the fit parameters are necessary to estimate the concentration. The suggested method has been used in field measurements for quantification of sulfur dioxide, $\mathrm{SO}_{2}$, in a desulphurization pilot unit in south Sweden [97].

In order to illustrate this approach, the LDA is calculated with only three $\mathrm{NO}_{\mathrm{x}}$ concentrations, i.e., 50/5, 125/12.5, and 200/20 ppm as shown in Fig. 8.7. The obtained LDA coefficients from this are then used for projection of the left-out concentrations, i.e., 75/7.5, 100/10, 150/15, and 175/17.5 ppm $\mathrm{NO}_{\mathrm{x}}$, and are marked with semi-solid symbols in Fig. 8.7a. The value of the centroids from only the three training groups is fitted by a second order polynomial, cf. Fig. 8.7b. The accuracy of the determination of the $\mathrm{NO}_{\mathrm{x}}$ concentrations can be estimated by the scatter of the 
projected groups. For the training concentrations the uncertainty is approximately $15 \mathrm{ppm}$ whereas for the test $\mathrm{NO}_{\mathrm{x}}$ concentrations it is rather $25 \mathrm{ppm}$.

In general, quantification of $\mathrm{NO}_{\mathrm{x}}$ by LDA together with a polynomial fit of group centroids is possible but LDA itself is rather suitable for discrimination of discrete groups than predicting concentrations. Therefore, Partial Least Squares Regression, PLSR, is studied additionally. The same data as before for calculating the LDA are used to build the regression model.
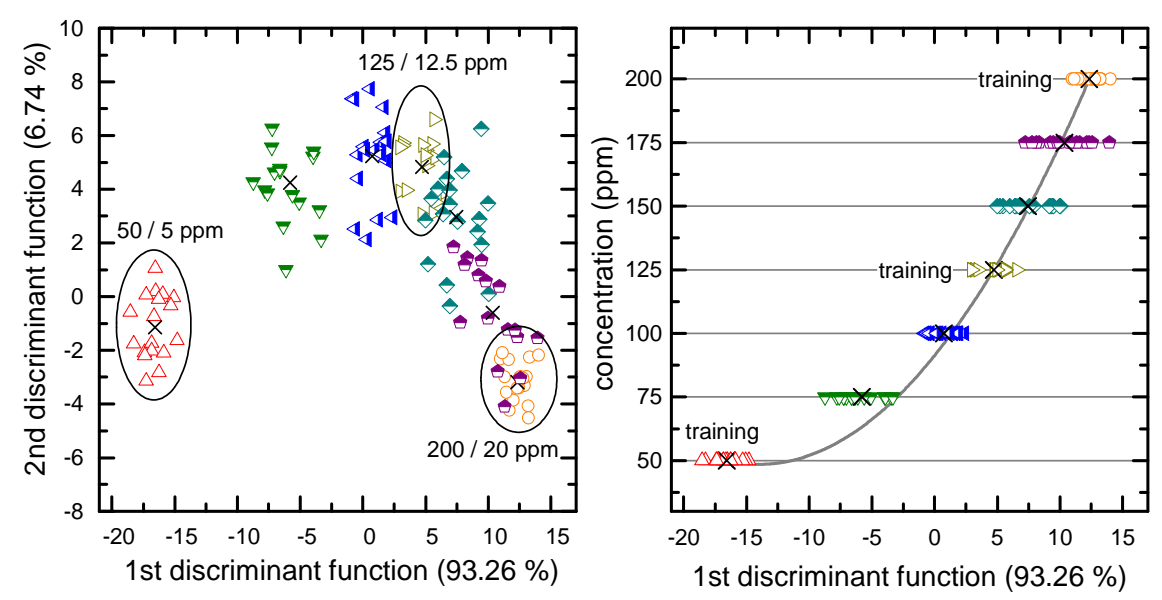

Fig. 8.7 (a) LDA scatter plot when the LDA is trained with only three $\mathrm{NO}_{\mathrm{x}}$ concentrations, i.e., 50/5, 125/12.5, and 200/20 ppm (solid symbols). "Unknown" concentrations, i.e., $75 / 7.5,100 / 10,150 / 15$, and 175/17.5 ppm, are projected using the obtained LDA coefficients (semi-solid symbols). (b) Fit of the centroids of the training groups over DF1 and projection of "unknown" concentrations.

The resulting regression plot when nine components are used is given in Fig. 8.8. The number of components was chosen based on the minimal cross-validation rate. The standard deviation of the training groups in the regression model, cf. Fig. 8.8a, is approximately $25 \mathrm{ppm}$. The performance of the regression model is illustrated in Fig. $8.8 \mathrm{~b}$ where only three $\mathrm{NO}_{\mathrm{x}}$ concentrations, i.e., 50/5, 125/12.5, and 200/20 ppm, were used for building the model. The left-out concentrations are used as test data and are marked by red solid symbols in the regression plot. The uncertainty is of the same magnitude as for the LDA with the fit function shown in Fig. 8.7b. However, a regression model has the advantage that it is more suitable since regression algorithms are designed for the prediction of concentrations while LDA does only 
discrimination of typically different gases. Thus, LDA requires an additional fit function to allow determination of concentrations. In the example given in Fig. 8.7b, a second order polynomial fit is needed to fit the training data sufficiently good whereas the PLSR model, cf. Fig. 8.8b, does a linear approximation. In general, the higher the order of the model, the less is the generalizability / stability.
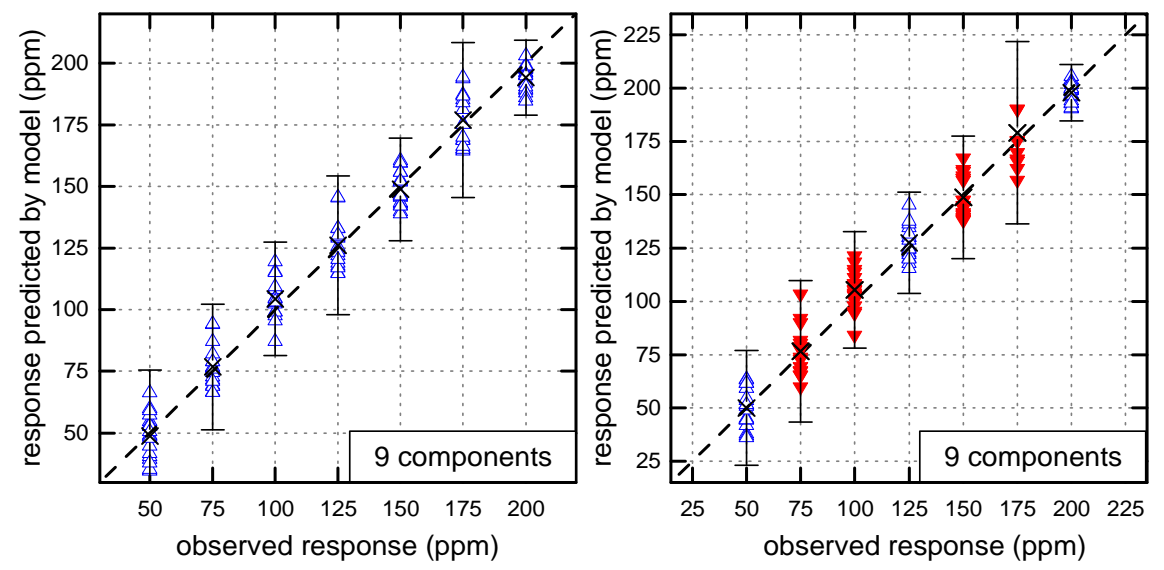

Fig. $8.8 \quad$ (a) Regression plot of a PLSR model build by the seven $\mathrm{NO}_{x}$ concentrations when nine components are used, and (b) when only three concentrations are used for building the model (blue open symbols). Projected "unknown" concentrations are marked with red solid symbols.

\subsubsection{Suppression of a Changing Background}

In the previous section, it is assumed that the background remains constant during the measurement. This, however, is usually not the case in real applications. Since the chosen background is rather complex, direct $\mathrm{NO}_{\mathrm{x}}$ quantification is not possible. In order to overcome this issue, a hierarchical strategy as described in Section 7.4.3 and the test gas profile shown in Fig. 8.1 are used. The discrimination strategy consists of two decision layers. First, the background is estimated, and then, based on this decision, a second training set is used for determination of the $\mathrm{NO}_{\mathrm{x}}$ concentration. The procedure of a hierarchical strategy is shown in Fig. 8.9.

As mentioned earlier the training set should contain as much information as possible in order to allow a robust discrimination. For the first decision, i.e., determination of the background mixture, an LDA model is built based on the three mixtures in which 
the concentration of one of the background gases, i.e., $\mathrm{NH}_{3}, \mathrm{C}_{2} \mathrm{H}_{4}$, and $\mathrm{CO}$, is changed compared to the standard mixture. In order to allow a robust discrimination, each group needs to contain the lowest and highest concentration of the corresponding background gas. Variations of the background concentration within this range should not affect the discrimination. Since the target gas is $\mathrm{NO}_{\mathrm{x}}$, it is also necessary to include data of $\mathrm{NO}_{\mathrm{x}}$ exposures in the different mixtures as shown in Fig. 8.9. It is also possible to build an LDA model with seven groups, i.e., the standard background and for each background gas the lowest and highest concentration. However, this approach has the drawback that the training groups represent discrete changes of the background. Prediction of the background based on interpolation of the projected data points between two training groups is very challenging and most often unstable. Thus, the hierarchical strategy as shown in Fig. 8.9 is used in the following. It should be mentioned that this strategy is suitable if only one of the background gases changes at the time. Otherwise the hierarchical strategy needs to be adjusted.

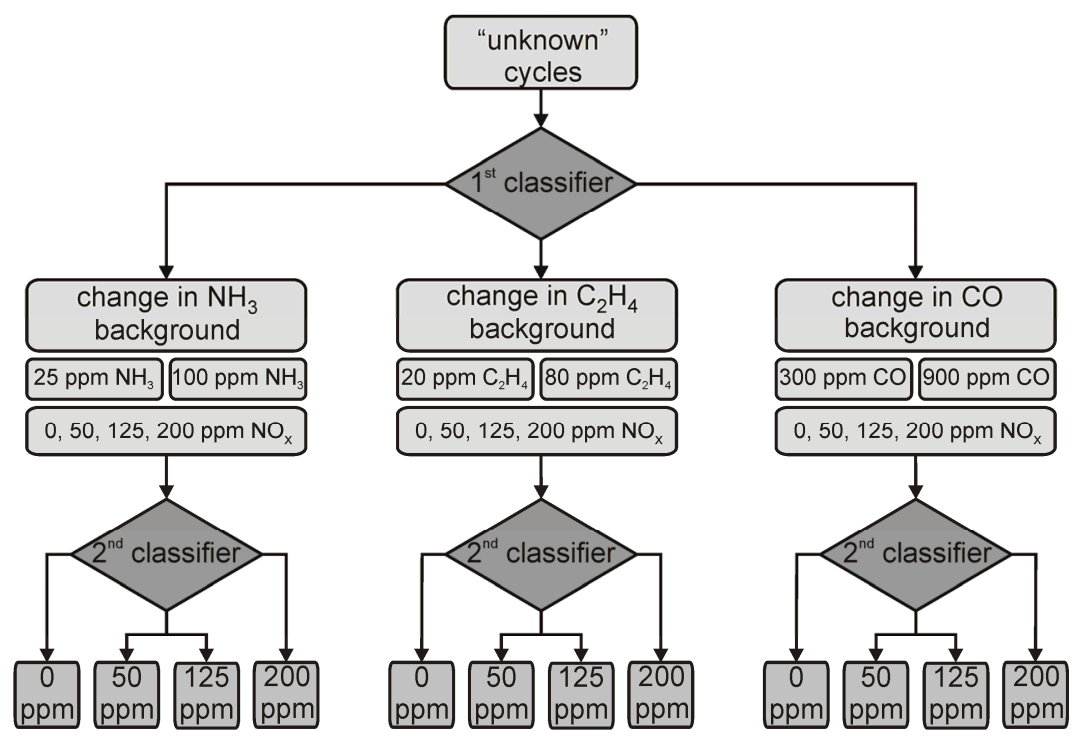

Fig. 8.9 Hierarchical strategy for $\mathrm{NO}_{\mathrm{x}}$ quantification in a varying background.

In the suggested hierarchical strategy, each of the three groups in the first layer covers basically the earlier defined standard mixture. Thus, these groups should not perfectly be separated. The LDA scatter plot showing the discrimination of the three 
background groups is given in Fig. 8.10a. There is almost no overlapping of the different background groups. This can be explained because LDA as a supervised method actively tries to separate the groups and thus, focuses on the group differences. Additionally, sensor drift during the measurement (the measurement lasts more than 70 h, cf. Fig. 8.1) cannot entirely be excluded. An effective way to compensate for sensor drift is extended training and discussed in Section 8.3.6 $6^{32}$.
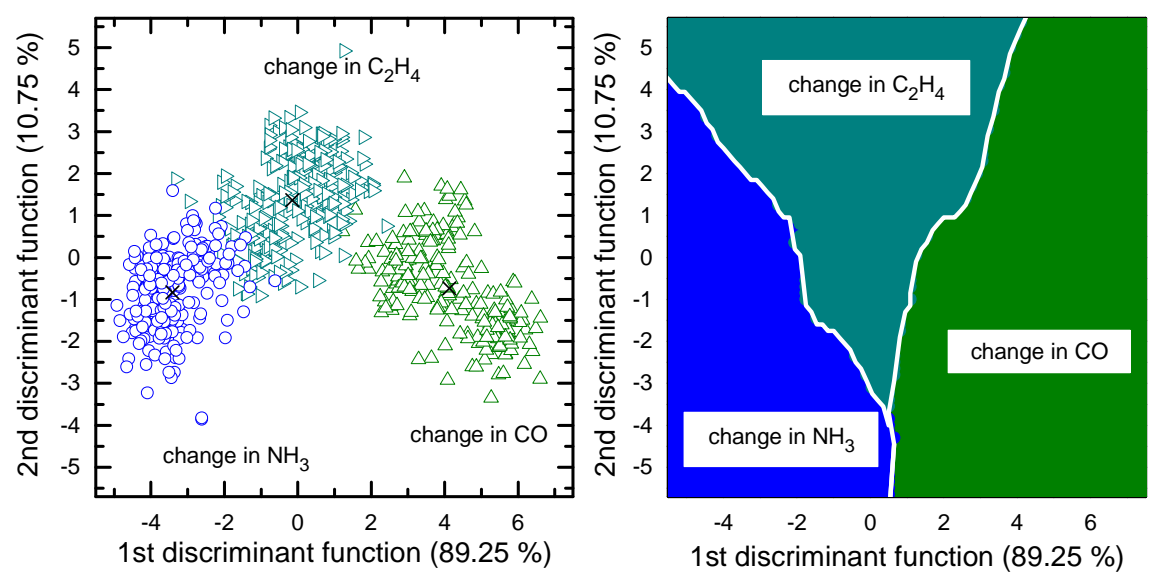

Fig. 8.10 (a) LDA scatter plot showing the discrimination of backgrounds. Each group contains four $\mathrm{NO}_{\mathrm{x}}$ concentrations, i.e., 0 , 50/5, 125/12.5, and 200/20 ppm in two different backgrounds, and (b) territorial plot when a $\mathrm{kNN}$-classifier is used with $\mathrm{k}=5$.

In general, the quality of this discrimination is not as critical since the first decision in the hierarchical strategy is only an estimation of the background. Overlapping of two groups means that it does not matter which data set is chosen for the quantification of $\mathrm{NO}_{\mathrm{x}}$ since the influence of a changing background is not high. In Fig. $8.10 \mathrm{~b}$ the corresponding territorial plot when using a $k$-Nearest Neighbor, kNN, classifier with $k$ equals 5 is used. The different territorials in the plot indicated how projected points would be classified. Unknown data points, i.e., data of unknown $\mathrm{NO}_{\mathrm{x}}$ concentration in an unknown background, are projected based on the LDA model obtained by the discrimination shown in Fig. 8.10a. Since all three groups cover the standard background, $\mathrm{NO}_{\mathrm{x}}$ data in this background would ideally be projected in between the three groups, i.e., close to the overall group centroid.

\footnotetext{
${ }^{32}$ When merging two measurements as described in Section 8.3.6, the resulting LDA scatter plot looks indeed very similar to Fig. 8.10a.
} 
However, in practice there are always slight variations so that the projected data point will be located closer to one of the groups. Again, this first decision is just an approximation of the background and the actual $\mathrm{NO}_{\mathrm{x}}$ quantification is done by the second decision. As an example, data of 50/5, 125/12.5, and 200/20 ppm $\mathrm{NO}_{\mathrm{x}}$ in a mixture with a higher $\mathrm{NH}_{3}$ concentration, i.e., $75 \mathrm{ppm}$, are used. The projection is shown in Fig. 8.11. Although, the test data are not contained in the training, the projected data points are located only in the training group "change in $\mathrm{NH}_{3}$ ".

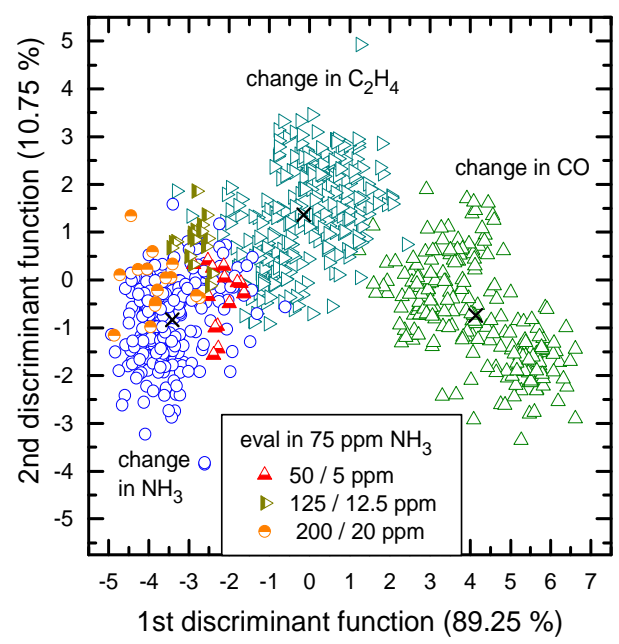

Fig. 8.11 LDA scatter plot showing the discrimination of backgrounds. Projected groups of "unknown" $\mathrm{NO}_{\mathrm{x}}$ concentrations, i.e., 50/5, 125/12.5, and 200/20 ppm $\mathrm{NO}_{\mathrm{x}}$ in a background with an increased $\mathrm{NH}_{3}$ concentration, are marked with semi-solid symbols.

Classification of the projected points is done by a k-Nearest Neighbor classifier with $\mathrm{k}=5$. The overall correct classification rate is almost $94 \%$ as shown in Table 9 .

Table 9 Correct classification rate obtained by a $\mathrm{kNN}$-classifier with $\mathrm{k}=5$.

\begin{tabular}{|c|c|c|}
\hline group & classified as & classification rate \\
\hline overall & --- & $93.7 \%$ \\
\hline 50 / 5 ppm $\mathrm{NO}_{x}$ in mixture with $75 \mathrm{ppm} \mathrm{NH}_{3}$ & change in $\mathrm{NH}_{3}$ & $85.7 \%$ \\
\hline $125 / 12.5 \mathrm{ppm} \mathrm{NO}_{\mathrm{x}}$ in mixture with $75 \mathrm{ppm} \mathrm{NH}_{3}$ & change in $\mathrm{NH}_{3}$ & $94.4 \%$ \\
\hline $200 / 20 \mathrm{ppm} \mathrm{NO}_{\mathrm{x}}$ in mixture with $75 \mathrm{ppm} \mathrm{NH}_{3}$ & change in $\mathrm{NH}_{3}$ & $100 \%$ \\
\hline
\end{tabular}


The higher the concentration, the higher the classification rate in the given example. However, as mentioned earlier, the first decision is just an estimation of the background mixture and misclassification is tolerable.

For the second decision in the hierarchical strategy, cf. Fig. 8.9, a training set for $\mathrm{NO}_{\mathrm{x}}$ quantification in a background with changed $\mathrm{NH}_{3}$ concentration needs to be used. In case of the suggested two layer hierarchical strategy, it is important that the training set for the second decision needs to consist of $\mathrm{NO}_{\mathrm{x}}$ data in two mixtures pursuant to the first decision: One background where the $\mathrm{NH}_{3}$ concentration is lower, i.e., $25 \mathrm{ppm}$, and one where the $\mathrm{NH}_{3}$ concentration is higher, i.e., $100 \mathrm{ppm}$, compared to the standard mixture. The unknown $\mathrm{NO}_{\mathrm{x}}$ data are then projected using this training set. Fig. 8.12 shows the discrimination of four $\mathrm{NO}_{\mathrm{x}}$ concentrations (open symbols), i.e., the quantification with a changed $\mathrm{NH}_{3}$ background, i.e., $25 \mathrm{ppm}$ and $100 \mathrm{ppm}$.

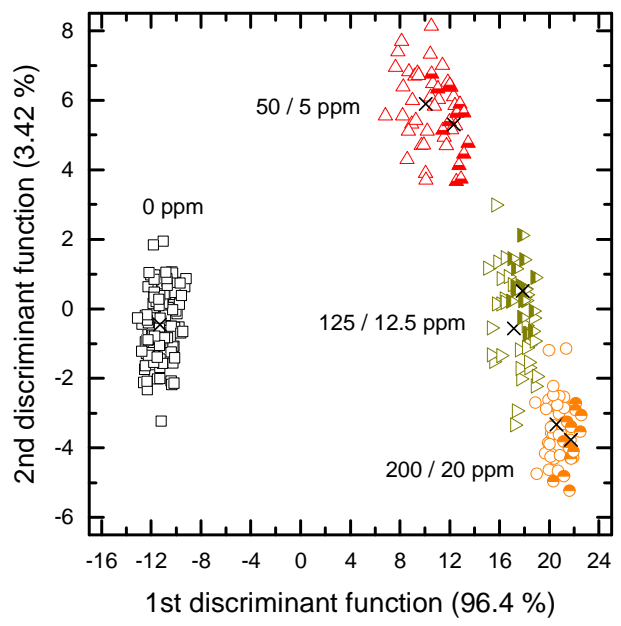

Fig. 8.12 LDA scatter plot showing the discrimination of four $\mathrm{NO}_{\mathrm{x}}$ concentrations (open symbols) in a background mixture with deviating two $\mathrm{NH}_{3}$ concentrations, i.e., $25 \mathrm{ppm}$ and $100 \mathrm{ppm}$, from the standard mixture. Projection of groups of "unknown" $\mathrm{NO}_{\mathrm{x}}$ concentrations, i.e., 50/5, 125/12.5, and 200/20 ppm in 75 ppm $\mathrm{NH}_{3}$ are marked with semi-solid symbols.

Projected data are marked by semi-open symbols. It can be observed that the projected groups are located very close to their corresponding training group and an overall correct classification rate of $100 \%$ is achieved. 
Again, the background mixture of the unknown groups is not contained in the training but quantification of $\mathrm{NO}_{\mathrm{x}}$ is still possible. The test gas profile, cf. Fig. 8.1, which is used for validating the suggested hierarchical strategy contains only four $\mathrm{NO}_{\mathrm{x}}$ concentrations per background mixture to keep the measurement time rather short. Nevertheless, in principle, quantification with the seven $\mathrm{NO}_{\mathrm{x}}$ concentrations from the standard background should be possible although, the accuracy is probably lower due to the scatter of the training groups.

\subsubsection{Conclusion $\mathrm{NO}_{x}$ Detection}

In this sub-chapter, gas-sensitive SiC-FETs with platinum gates were used for quantification of $\mathrm{NO}_{\mathrm{x}}$ in a background of other typical exhaust gases and ammonia. Results for iridium gates can be found in [296], [273]. A suitable temperature cycle was developed based on temperatures of maximum sensor response towards $\mathrm{NO}_{\mathrm{x}}$ in an exhaust mixture. For that, the operating temperature was ramped slowly from $100{ }^{\circ} \mathrm{C}$ up to $400{ }^{\circ} \mathrm{C}$ in a mixture of carbon monoxide, ethene, and ammonia, i.e., without $\mathrm{NO}_{x}$, and with additional $\mathrm{NO}$ and $\mathrm{NO}_{2}$. By looking at the difference signal between $\mathrm{NO} / \mathrm{NO}_{2}$ and the mixture, points of maximum sensor response and with a high gradient were chosen to build the cycle.

Quantification of $\mathrm{NO}_{\mathrm{x}}$ in a standard background, i.e., $600 \mathrm{ppm} \mathrm{CO}, 50 \mathrm{ppm} \mathrm{C}_{2} \mathrm{H}_{4}$,

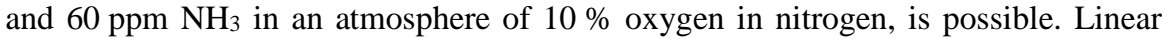
Discriminant Analysis and Partial Least Squares Regression were studied for data evaluation, and both algorithms allow prediction of unknown $\mathrm{NO}_{\mathrm{x}}$ concentration. LDA leads to slightly better results but PLSR is more suitable for monitoring of continuously changing $\mathrm{NO}_{\mathrm{x}}$ concentrations due to the linearity of the model and the directly provided analog (continuous) output value.

The influence of a varying background can be compensated by a hierarchical strategy which first estimates the kind of background, and then quantifies $\mathrm{NO}_{\mathrm{x}}$. It was shown by lab measurements that it is sufficient to build the model only with the minimum and maximum concentrations of each background gas to make it robust for any variations within this range. Quantification of $\mathrm{NO}_{\mathrm{x}}$ in the determined background is possible. The suggested hierarchical approach has been used to develop a sulfur dioxide sensor, $\mathrm{SO}_{2}$, for control of a desulfurization pilot unit in a power point [97]. 
In this field measurement more than one gas changes at a time and with a slightly adapted hierarchical strategy, quantification of $\mathrm{SO}_{2}$ is possible.

Hence, for complex applications a hierarchical strategy is suitable but the number of decision layers in the strategy should be kept as low as possible since every layer increases the necessary amount of training data considerably. Additionally, the stability of the system decreases with a higher number of layers. Thus, for each decision as much of information, i.e., changing parameters, as possible should be included in order to increase the stability. 


\subsection{SiC-FETs for Indoor Air Quality Applications}

In this sub-chapter, silicon carbide based field effect transistors, SiC-FETs, are studied for indoor air quality applications where volatile organic compounds, VOCs, need to be detected in the low parts-per-billion, ppb, range. In the first section, i.e., Section 8.2.1, the applied temperature cycles and relative sensor responses are presented. Section 8.2.2 deals with the discrimination of formaldehyde, naphthalene, and benzene, which are typical representatives for indoor air pollutants. Discrimination is done by Linear Discriminant Analysis, LDA, and Partial Least Squares - Discriminant Analysis, PLS-DA. Quantification of VOCs is performed by LDA and Partial Least Squares Regression, PLSR, as discussed in Section 8.2.3. After that, VOC detection in a background of an interfering gas, i.e., ethanol is studied, cf. Section 8.2.4, followed by a conclusion in Section 8.2.5

This subchapter is mainly based on two conference contributions [297], [298] and two journal articles [47], [299].

\subsubsection{Detection of Volatile Organic Compounds}

In this study, three-terminal depletion type SiC-FET sensors with either $25 \mathrm{~nm}$ thick porous platinum or $30 \mathrm{~nm}$ thick porous iridium as described in Section 6.2.3 are used. The sensors are operated in the linear region at the onset of saturation allowing a constant current operation. The reason for choosing the linear region instead of the saturation region is motivated by the measurement hardware rather than by the physics of the sensor. As discussed in Section 7.1, the saturation region theoretically offers a higher sensitivity of the SiC-FETs but requires a constant voltage operation. Due to the fact that the used hardware boards only use 10 bit ADC for measuring the current, but a 14 bit ADC for measuring the voltage, a higher resolution can be achieved when the sensor is operated in a constant current mode, which is not possible in saturation. Since the expected sensor response for low concentrations of VOCs is rather small, a constant current operation at the onset of saturation is 
chosen. Preliminary results with a modified hardware board and a SiC-FET operated in the saturation region can be found in the master thesis of Manuel Bastuck [276].

Silicon carbide field effect transistors have so far not been studied for the detection of low concentrations of volatile organic compounds. Thus, rather simple temperature cycles are used for the first studies. Each cycle consists of three temperature plateaus each of which is $15 \mathrm{~s}$ long as shown in Fig. 8.13. For platinumgate SiC-FETs $220^{\circ} \mathrm{C}, 200^{\circ} \mathrm{C}$, and $180^{\circ} \mathrm{C}$, and for iridium-gate SiC-FETs $350{ }^{\circ} \mathrm{C}$, $300{ }^{\circ} \mathrm{C}$, and $250{ }^{\circ} \mathrm{C}$ are chosen due to the fact that these sensors usually show good sensitivity to various gases in this temperature range. Since the sensors should be operated at the onset of saturation, which starts around 1 V, cf. Fig. 6.9, the possible temperature range is limited since a change in temperature strongly affects the baseline of the sensor. Anyhow, the cycles are used for discrimination purposes, cf. Section 8.2.2, rather than to find the optimal operating temperature - at least in this sub-chapter, in which the suitability of SiC-FETs for indoor air quality, IAQ, applications is studied. The baseline can be adjusted by the set drain current and the applied gate and/or substrate bias. However, since the gate bias also affects the sensitivity of the sensors, cf. Section 4.3, it is kept grounded in order to limit the number of open parameters for the first studies. As shown in Fig. 8.13a the sensor signal (black circles) follows the temperature change with a maximum of $0.95 \mathrm{~V}$ at $220^{\circ} \mathrm{C}$ and a minimum at around $0.55 \mathrm{~V}$ at $180^{\circ} \mathrm{C}$.
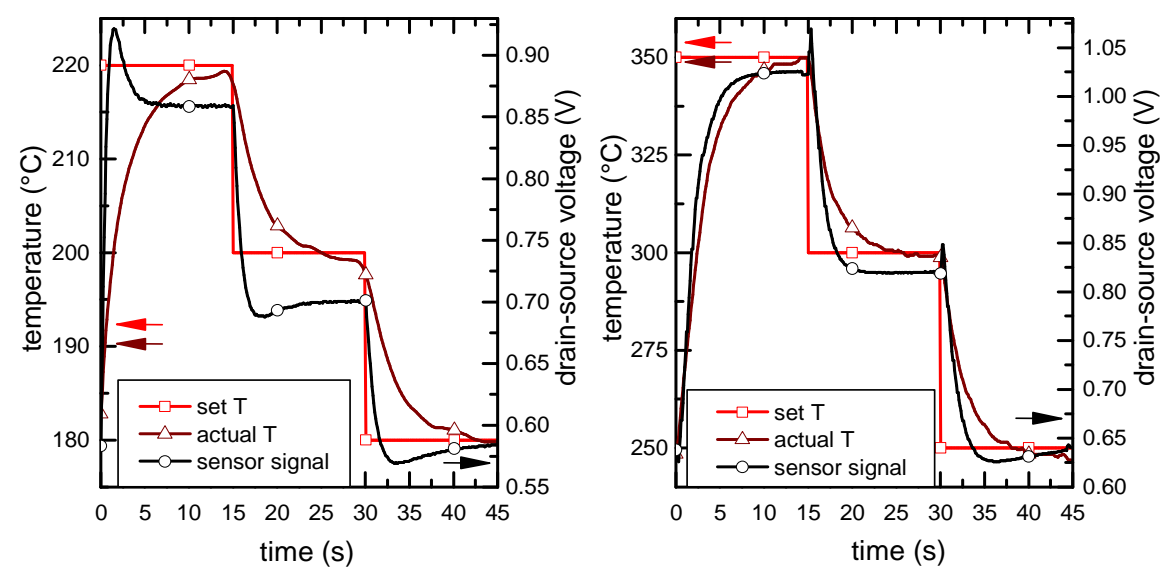

Fig. 8.13 Applied temperature cycles with set and actual temperatures as well as the sensor signal in synthetic air. (a) Platinum-gate SiC-FET, and (b) iridium-gate SiC-FET. 
Based on the basics of a SiC-FET, the sensor response in the linear region depends on $V_{D S}$, cf. Eq. (7.1), which means the sensor response is typically highest for the highest temperature ${ }^{33}$. Again, the sensor signal follows the temperature change and the baseline is in the range of $0.6 \mathrm{~V}$ to $1 \mathrm{~V}$. The current set for Pt-gate SiC-FETs is, depending on the sample, between $40 \mu \mathrm{A}$ and $100 \mu \mathrm{A}$ whereas for Ir-gates the current is rather low, i.e., between $20 \mu \mathrm{A}$ and $40 \mu \mathrm{A}$ due to the low saturation current.

As typical VOCs formaldehyde, naphthalene, and benzene are chosen and the concentration ranges are defined with respect to the current legal threshold values. Ethanol is chosen as a typical interferent with a concentration range from $0.5 \mathrm{ppm}$ to $2 \mathrm{ppm}$ and is thus a few magnitudes higher than the concentrations of the target VOCs. The studied gas profile contains at least five concentrations for each VOC, for formaldehyde $25,50,75,100$, and $200 \mathrm{ppb}$, for naphthalene $2.5,5,10,20$, and $40 \mathrm{ppb}$, and for benzene $0.5,1.5,2.5,3.5$, and $4.5 \mathrm{ppb}$. In order to supply these low concentrations of VOCs, the gas mixing system presented in Section 6.1.2 is used.

The relative sensor responses $S=\frac{V_{D S_{a i r}}-V_{D S} \text { gas }}{V_{D S_{a i r}}}$ for naphthalene for Pt- and Ir-gate SiC-FETs at the highest studied temperatures, i.e., $220^{\circ} \mathrm{C}$ and $350{ }^{\circ} \mathrm{C}$, are shown in Fig. 8.14.
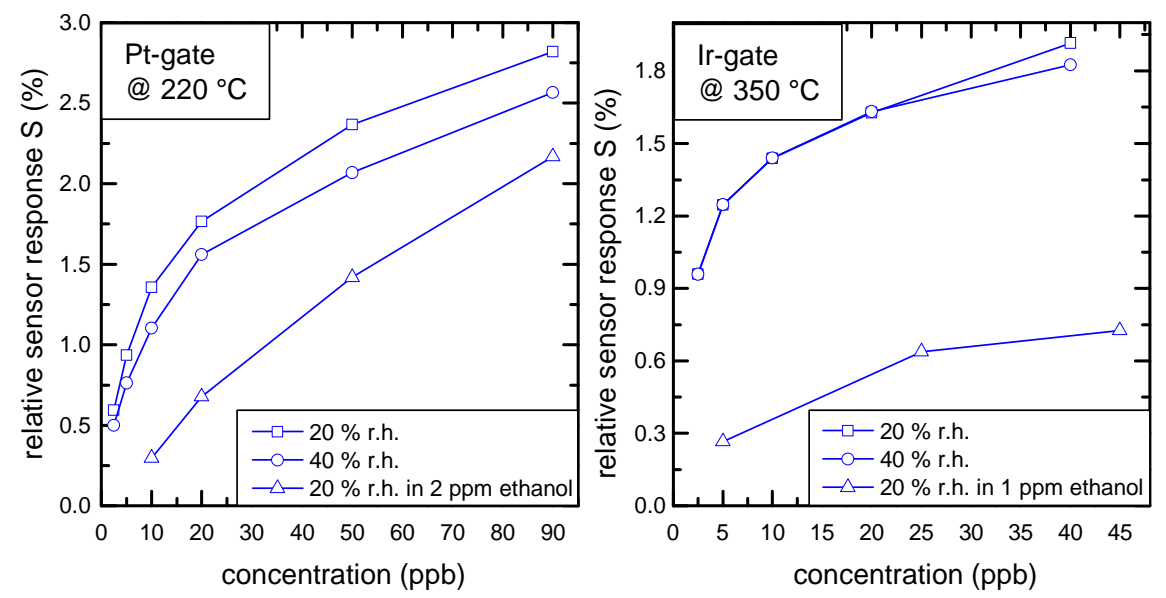

Fig. 8.14 Relative sensor response for naphthalene at two humidity levels and in a mixture with ethanol. (a) Platinum-gate SiC-FET, adapted from [47], and (b) iridium-gate SiC-FET.

\footnotetext{
${ }^{33}$ This is true for a limited temperature range in which the sensor shows a reasonable sensitivity to the target gases.
} 
The relative sensor response is slightly higher for platinum whereas iridium is less sensitive to relative humidity. In general, the influence of relative humidity on the sensor response for both gate metallizations seems to be rather small. In a high background of $2 \mathrm{ppm}$ ethanol detection of naphthalene is still possible but the sensitivity is decreased.

The relative sensor response for formaldehyde is lower than for naphthalene although, the concentrations of interest are higher, cf. Fig. 8.15a. The influence of relative humidity is rather low as before. In Fig. 8.15a also the sensor response for a Pt-gate SiC-FET at $200{ }^{\circ} \mathrm{C}$ is shown. The corresponding response is lower than for $220{ }^{\circ} \mathrm{C}$ which is probably because the baseline, i.e., $V_{D S}$, is lower ${ }^{34}$, cf. Fig. 8.13. Fig. $8.15 \mathrm{~b}$ shows the sensor response of a Pt-gate at $220{ }^{\circ} \mathrm{C}$ for five concentrations of benzene. A detection limit of approximately $1 \mathrm{ppb}$ can be achieved. In general, the response is rather small but on the other hand the concentrations which need to be detected are low as well.
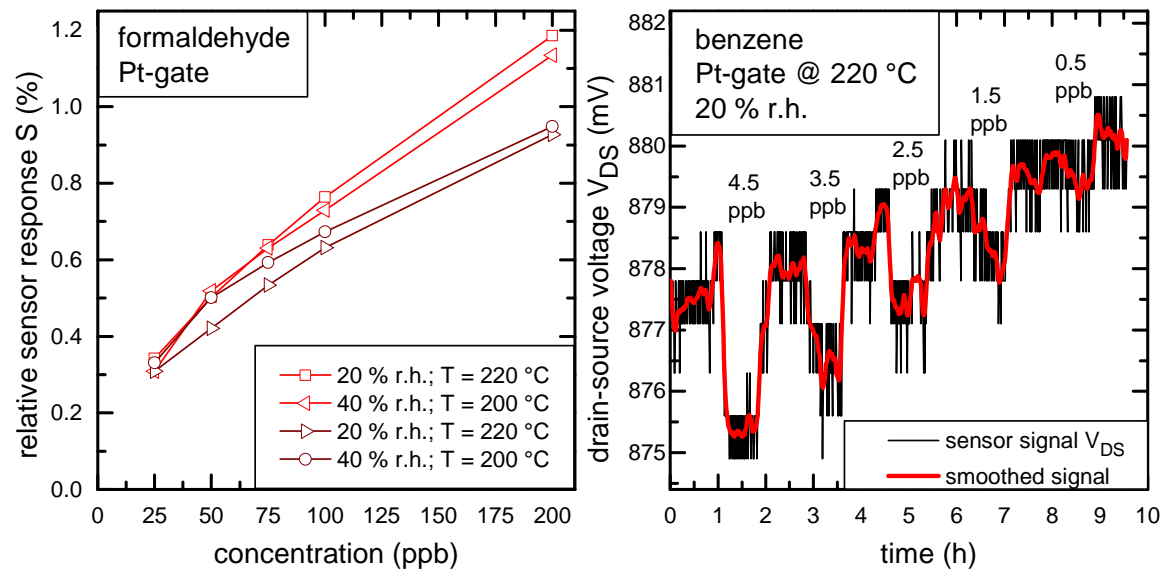

Fig. 8.15 (a) Relative sensor response for formaldehyde at two humidity levels and at two temperatures, and (b) sensor response for benzene. Reproduced from [47] with kind permission from IEEE.

The sensor responses are also depended on the studied sensor sample ${ }^{35}$. Thus, the results presented in this section, i.e., Fig. 8.14 and Fig. 8.15, are rather qualitative

\footnotetext{
${ }^{34}$ This is only true for the linear region.

${ }^{35}$ Depending on the location of the sensor samples on the wafer, i.e., in the center or at the edge of the wafer, the threshold voltage differs from sample to sample due to the manufacturing process [259]. For instance, one sensor was studied which
} 
than quantitative results. Detection of the chosen VOCs in the relevant concentration range is possible and the influence of relative humidity is rather small.

In addition to the results presented here, Puglisi et al. studied elaborately the sensor response of iridium-gate SiC-FETs operated at a constant temperature [300], [56].

\subsubsection{Discrimination of Volatile Organic Compounds}

The temperature cycles presented in the last section are used for discriminating typical VOCs like formaldehyde, naphthalene, and benzene. The sensor response is normalized first by setting the cycle mean to one. Fig. 8.16 shows the difference normalized signal of a Pt-gate SiC-FET for $100 \mathrm{ppb}$ formaldehyde, $20 \mathrm{ppb}$ naphthalene, and $4.5 \mathrm{ppb}$ benzene in order to suppress the influence of temperature on the sensor baseline.

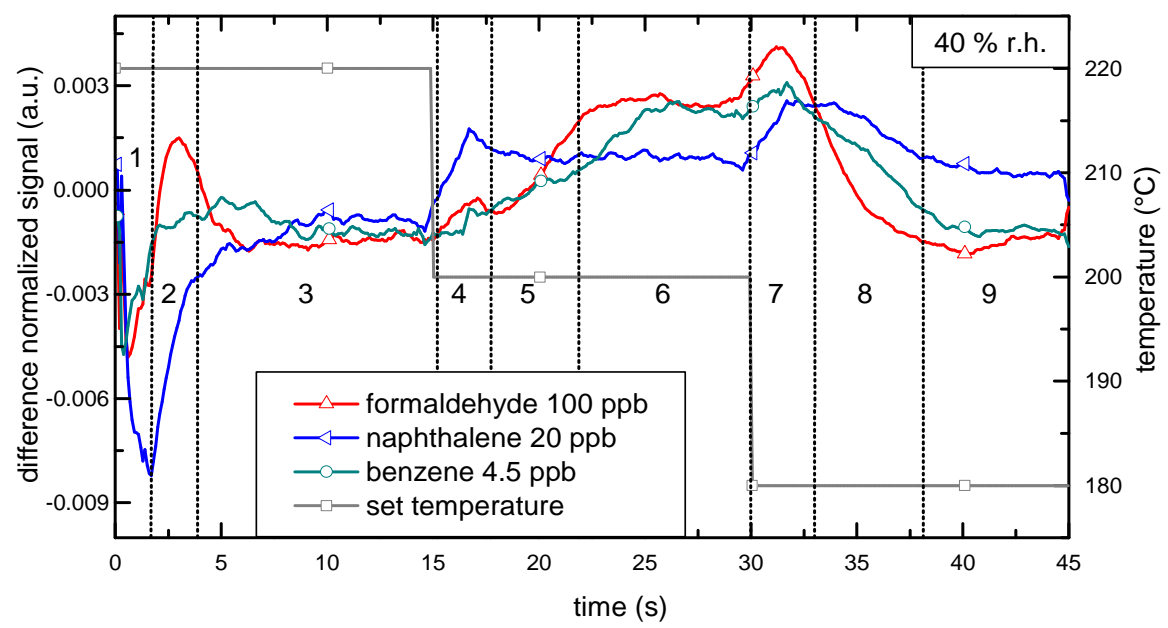

Fig. 8.16 Normalized difference signal of a Pt-gate SiC-FET for $100 \mathrm{ppb}$ formaldehyde, $20 \mathrm{ppb}$ naphthalene, and $4.5 \mathrm{ppb}$ benzene with $40 \%$ r.h. at $20{ }^{\circ} \mathrm{C}$. Nine intervals for feature extraction are marked. Adapted from [299].

As can be seen, the difference signal changes characteristically in certain parts of the cycle. Nine intervals are defined for the feature extraction. Especially, in intervals where the temperature changes, i.e., interval 1 and 2 , or 7 and 8 , the differences

had a four times higher relative sensor response but several other sensors showed relative response in the same order as shown in Fig. 8.15. 
between the curves, i.e., gases are high. As features mean values and slopes, i.e., best fit lines, are calculated. These 18 features are used as input for pattern recognition algorithms, like Linear Discriminant Analysis and Partial Least Squares Discriminant Analysis, which are used to discriminate VOCs in this section.

In the following, only platinum-gate SiC-FETs are considered since they offer a higher sensitivity as shown in Section 8.2.1.

Fig. 8.17 shows an LDA scatter plot showing the discrimination of several concentrations of formaldehyde, naphthalene, and benzene with $40 \%$ r.h.

A clear separation of the different gases with only little overlap is possible. Even the different concentrations of naphthalene and formaldehyde are located apart from each other. Only the lowest concentrations are close to the pure air group and overlap a bit with the low naphthalene concentrations. Benzene which leads to a small sensor response is separated from the other groups but closely located to the pure air group. It should be noted that the pure air groups is always located next to the group corresponding to the lowest concentration of a particular VOC. Higher concentrations are located further apart from the pure air, i.e., $0 \mathrm{ppb}$ group. This can be seen as indirect validation.

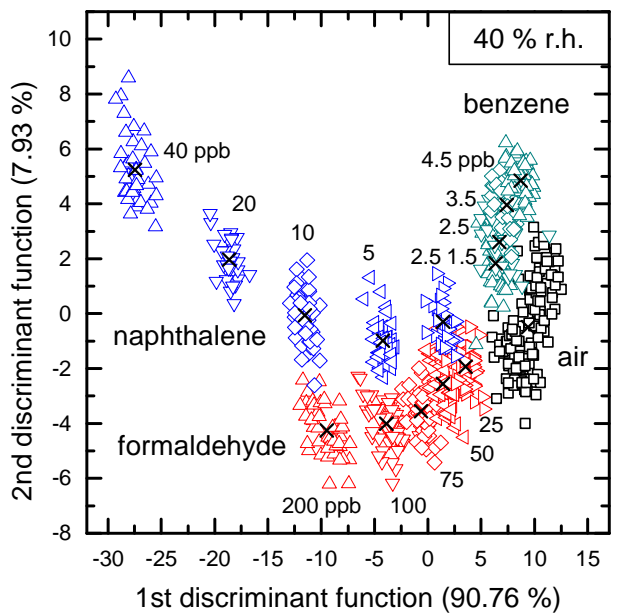

Fig. 8.17 LDA score plot showing the discrimination of several concentrations of formaldehyde, naphthalene, and benzene with $40 \%$ r.h. when a Pt-gate SiC-FET is used. 
Although, this scatter plot discriminates the three different VOC nicely, it is not optimal to determine a specific VOC since any change in humidity or concentration could easily lead to misclassification. A more stable and robust discrimination is achieved if parameters that can vary, e.g., humidity, are included in the training. For determination of target gases it is also beneficial to include several different concentrations in the training. Since the application behind the VOC studies is indoor air quality and on-demand ventilation as mentioned in the introduction, cf. Section 2.2, it is preferable to detect target gases at, or just above their threshold value. For instance, if the threshold value is not exceeded ventilation does not need to be switched on.

Therefore, it is conceivable to combine concentrations of all target gases which are lower than the corresponding threshold value in one group, e.g., a "below threshold" group. The current threshold value for formaldehyde is $80 \mathrm{ppb}$, for naphthalene is $5.7 \mathrm{ppb}$, and for benzene $1.5 \mathrm{ppb}$, cf. Section 2.2. In Fig. 8.18a the discrimination of the three target VOCs as well as the "below threshold" group is given. Each VOCgroup contains three concentrations, i.e., 75, 100, and $200 \mathrm{ppb}$ formaldehyde, 5, 10, and $20 \mathrm{ppb}$ naphthalene, and 2.5, 3.5, and $4.5 \mathrm{ppb}$ benzene, which are close to, or above the corresponding threshold value.
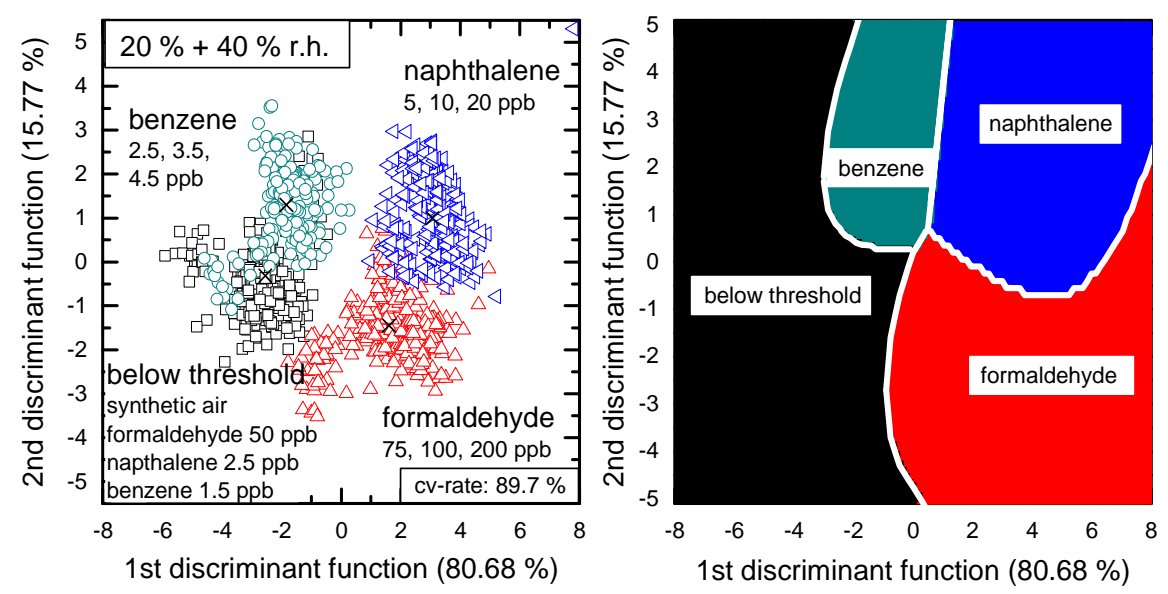

Fig. 8.18 (a) LDA scatter plot showing the discrimination of formaldehyde, naphthalene, benzene, and the "below threshold" group when a Pt-gate SiC-FET is used. (b) Territorial plot of the discrimination when a Mahalanobis distance classifier is used. Adopted from [299]. 
The "below threshold" group holds data of pure air, $50 \mathrm{ppb}$ formaldehyde, $2.5 \mathrm{ppb}$ naphthalene, and $1.5 \mathrm{ppb}$ benzene which is the current threshold. The discrimination contains also data at two different relative humidity levels, i.e., $20 \%$ and $40 \%$ r.h., in order to make the separation independent of the humidity, at least between these values. The scatter of the groups in the plot is rather high but a clear separation of the groups is obtained.

Leave-one-out cross-validation, LOOCV, with a Mahalanobis distance classifier leads to an overall correct classification rate of almost $90 \%$, cf. Table 10. Benzene overlaps a bit with other groups, which leads to a lower individual classification rate of $82 \%$. Again, the shown discrimination is very robust against changing humidity and also varying concentration of the target VOCs. Since (canonical) LDA does only projection, a separate classifier is needed.

Table 10 Leave-one-out cross-validation of the discrimination shown in Fig. 8.18a using a Mahalanobis distance classifier.

\begin{tabular}{lr}
\hline group & classification rate \\
\hline overall & $89.7 \%$ \\
below threshold & $89.9 \%$ \\
formaldehyde & $90.7 \%$ \\
naphthalene & $94.1 \%$ \\
benzene & $81.7 \%$ \\
\hline
\end{tabular}

Here, the Mahalanobis distance was used and the resulting territorial plot shows how "unknown" points would be classified, cf. Fig. 8.18. Determination of the target VOC and determining the concentration at the same time is not possible. Thus, a two layer hierarchical strategy, i.e., decision tree, as shown in Fig. 8.19 can be used. In a first step, the main air pollutant, i.e., the target VOC needs to be determined. After that, in a second step, quantification can be done if one of the target gases exceeds its corresponding threshold. Quantification of VOCs is discussed in the next section, i.e., Section 8.2.3. For a simple ventilation strategy it is sufficient to determine wherever one of the target VOCs exceeds its threshold. However, for smart ventilation, in which also the quality of the outdoor air is taken into account, a rough estimation of the concentration of the main pollutant is needed. For instance, if the indoor air is seriously polluted the ventilation should be stronger. 


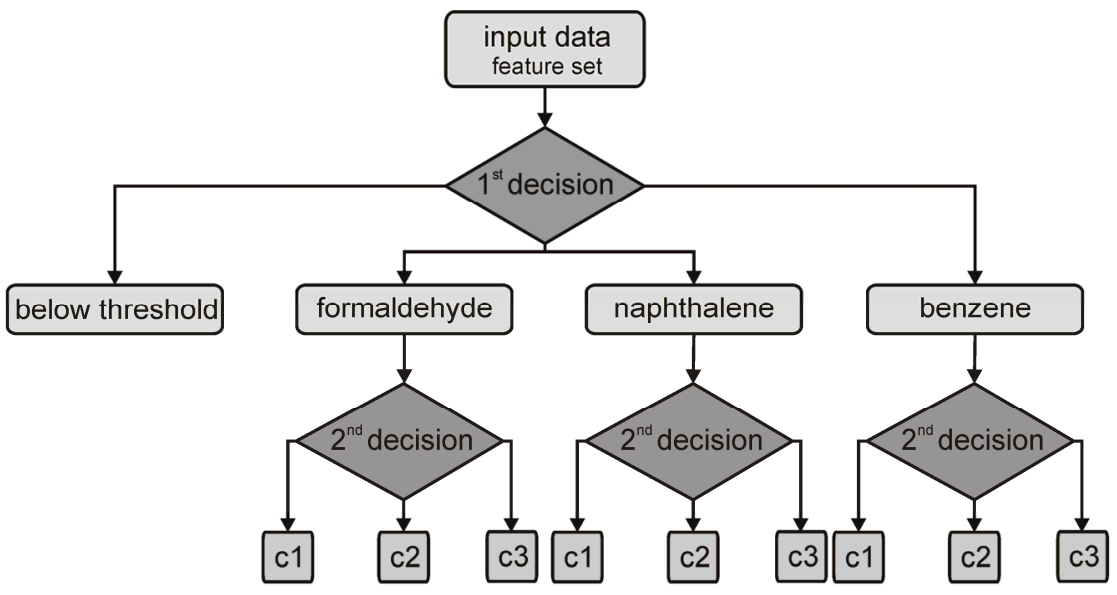

Fig. 8.19 Schematic of a two layer decision process for quantification of VOC.

Besides LDA which already achieves good results but requires an additional classifier, Partial Least Squares - Discriminant Analysis, PLS-DA, is studied as well. The advantage of PLS-DA over LDA is the direct classification and, in particular, the possibility to adjust the classifier easily.

The same data set as before, i.e., Fig. 8.18, is now used for PLS-DA. Fig. 8.20 shows the result of PLS-DA when 10 components were used. Since PLS-DA performs onevs-all classification, the result for the given problem with three VOCs and the "below threshold" class are four scatter plots, i.e., one per class. In the top left panel in Fig. 8.20 formaldehyde is separated from the other three classes which are treated by the algorithm as one single class. The coloring of the classes is only to show which gases might lead to misclassification. The classification rate for formaldehyde is $76 \%$, i.e., $76 \%$ of all formaldehyde data points are correctly classified as formaldehyde. Again, also this class contains three different concentrations at two levels of relative humidity, i.e., $20 \%$ and $40 \%$ r.h. The classification threshold can be set by the user which means that the classification rate can be adjusted. This is in particular interesting for the application, i.e., on demand ventilation control. With this adjustment the point at which ventilation should be switched on can be adjusted easily. Naphthalene and benzene are classified correctly by $99 \%$ and $90 \%$, respectively. Data points which are assigned to the "below threshold" group are classified correctly by $73 \%$. The main reason why the rate is so low is because of 
benzene. The overall-classification rate is $83.4 \%$ which is lower than that obtained using LDA with a Mahalanobis distance classifier (89.7\%). However, the independent adjustment of the classifier, for each group / class is probably not as easy for LDA.
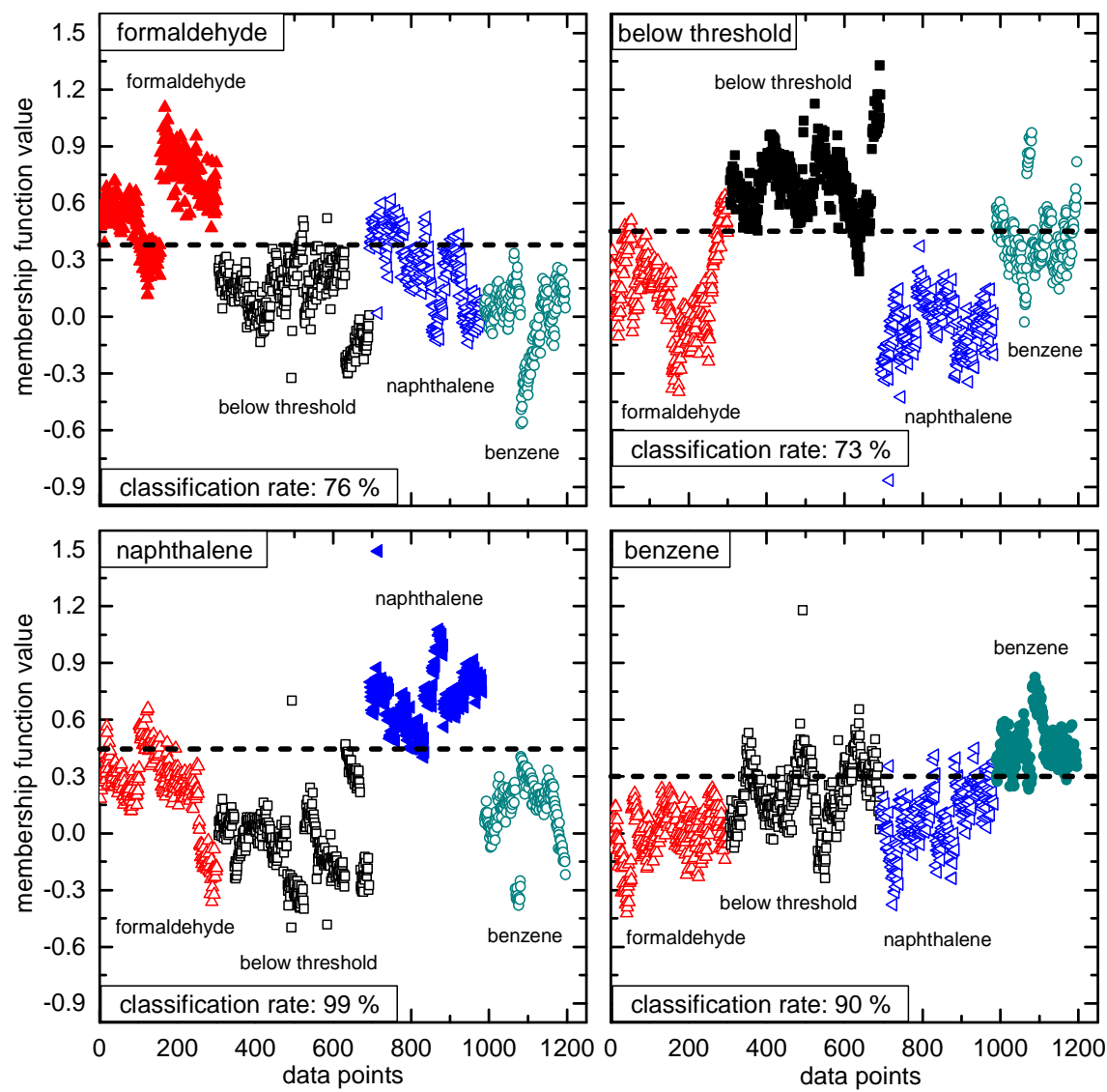

Fig. 8.20 PLS-DA scatter plots showing the separation of formaldehyde, naphthalene, benzene, and the "below threshold" group with combined humidity levels, i.e., $20 \%$ and $40 \%$ r.h. 10 components are used for the classification. Adopted from [299].

\subsubsection{Quantification of Volatile Organic Compounds}

After identification of the main air pollutant by LDA or PLS-DA, quantification is required as basis for determining the ventilation strategy. 
Fig. 8.21 shows the discrimination of different concentrations of formaldehyde. Determination of the concentration with $40 \%$ r.h. is possible; however the $75 \mathrm{ppb}$ and 100 ppb groups overlap a bit, cf. Fig. 8.21a. Discrimination is mainly along the first discriminant function. Leave-one-out cross-validation with a Mahalanobis distance classifier leads to a validation rate of $94.6 \%$. If two relative humidity levels are combined, i.e., $20 \%$ and $40 \%$ r.h., determination of the concentration is still possible, although, the importance of DF2 increases to $12.8 \%$. Thus, for correct identification of the concentration also DF2 is needed.
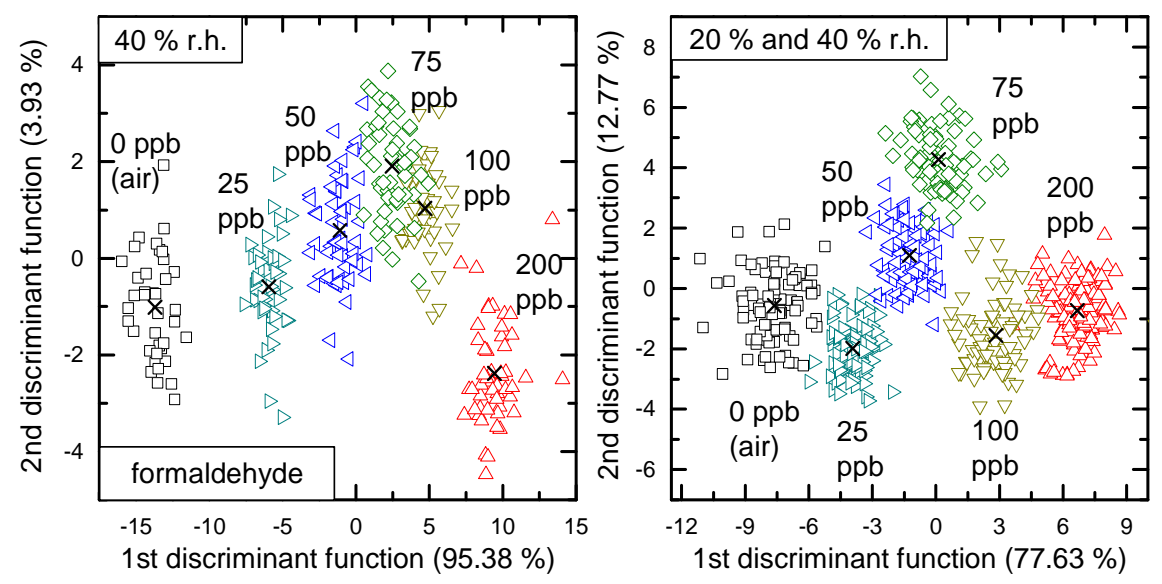

Fig. 8.21 LDA scatter plot showing the discrimination of different concentrations of formaldehyde. (a) With $40 \%$ r.h., and (b) with two combined levels of relative humidity, i.e., $20 \%$ and $40 \%$ r.h.

A possible explanation is probably the rather small relative sensor response of formaldehyde over a wide concentration range, cf. Section 8.2.1, which makes a discrimination challenging. Nevertheless, a cross-validation rate of $95.8 \%$ is achieved by LOOCV.

From a relative sensor response point of view, naphthalene looks more promising in terms of quantification. Fig. 8.22 shows the discrimination of different concentrations of naphthalene as well as the influence of relative humidity. First, different concentrations at $20 \%$ r.h. are discriminated (open symbols). As can be seen, the quality of the separation is high; the groups are compact with sufficient margin to their neighbors. The obtained LDA coefficients, i.e., the training, are in a next step applied to unknown data which are then projected into the same scatter plot 
(semi-solid symbols). As unknown data the same naphthalene concentrations are used but at $40 \%$ r.h. It can be observed that the projected groups are located close to their corresponding training group with almost the same DF1 values.

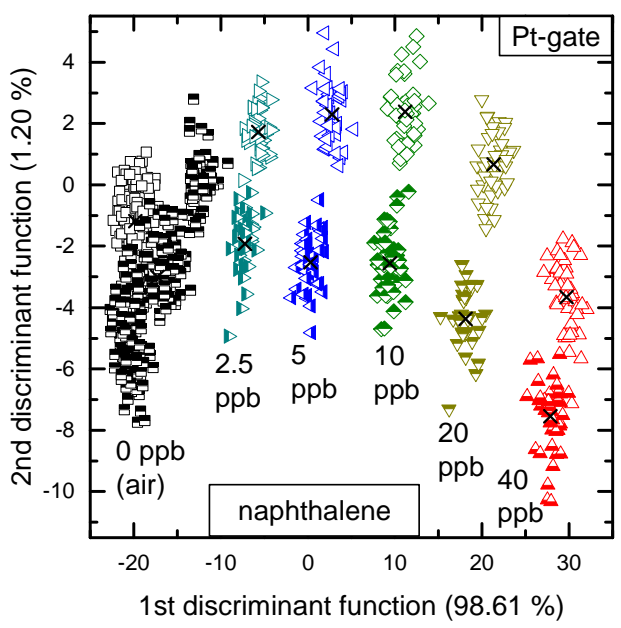

Fig. 8.22 LDA scatter plot showing the discrimination of different concentrations of naphthalene at $20 \%$ r.h. and the influence of humidity. First, different concentrations at $20 \%$ r.h. are discriminated (open symbols). Based on this, data at $40 \%$ r.h. are projected (semi-solid symbols). Adopted from [47].

A similar shift in the direction of DF2 for $40 \%$ r.h., which represents only $1.2 \%$ of all the discriminatory information, is visible. A Mahalanobis distance classifier achieves a correct classification rate of $96.1 \%$. Thus, the influence of humidity on the naphthalene quantification is rather small. However, in order to create a robust system, it is recommended to extend the training set, i.e., to combine data from both humidity levels. The discrimination independent of the humidity is given in Fig. 8.23. The scatter is slightly higher but there is no pronounced overlapping of groups. Determination of the concentration is possible by using only the value of DF1 and the distribution of the scores is illustrated by the histogram in Fig. 8.23.

So far, only different concentrations are discriminated but no classification of unknown concentrations, i.e., quantification. In Fig. 8.24 top panel, only three naphthalene concentrations, i.e., 0,5 , and $40 \mathrm{ppb}$, are used for calculating the LDA (open symbols). Again, two levels of relative humidity are combined. The reason for 
choosing these concentrations is that $5 \mathrm{ppb}$ is approximately the current legal limit and thus, also the threshold for ventilation control.

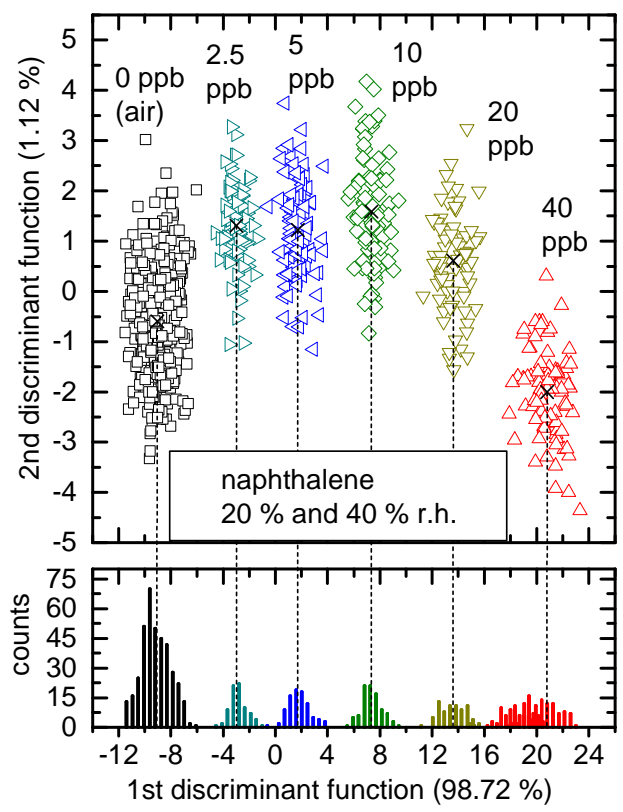

Fig. 8.23 Top: LDA scatter plot showing the discrimination of different concentrations of naphthalene independent of the humidity level. Bottom: histogram showing the distribution of the scores, i.e., cycles, along DF1. Adopted from [298], [299].

The other two concentrations, i.e., 0 and $40 \mathrm{ppb}$, are used as sampling points, one below and one above the threshold. The left-out concentrations are used as unknown data and are projected into the same scatter plot by using the obtained LDA coefficients (semi-solid symbols). It can be observed that the projected groups fit perfectly to the training data. The dashed line represents a possible threshold for ventilation control. Projected data points with a DF1 value smaller than 2.5 would mean "no ventilation needed", whereas points with a greater DF1 value would cause start of ventilation. In order to give a rough estimation of the concentration, the centroids of the training data can be fitted by a second order polynomial. The corresponding plot concentration-vs-DF1-value is given in Fig. 8.24 bottom panel. In this plot, the projected data are again marked by semi-solid symbols. The scatter of the groups along the abscissa is a measure of the uncertainty of the determination of the concentration. The suggested method is of course not very accurate but since the 
application is ventilation control, only an estimation of the concentration is sufficient.
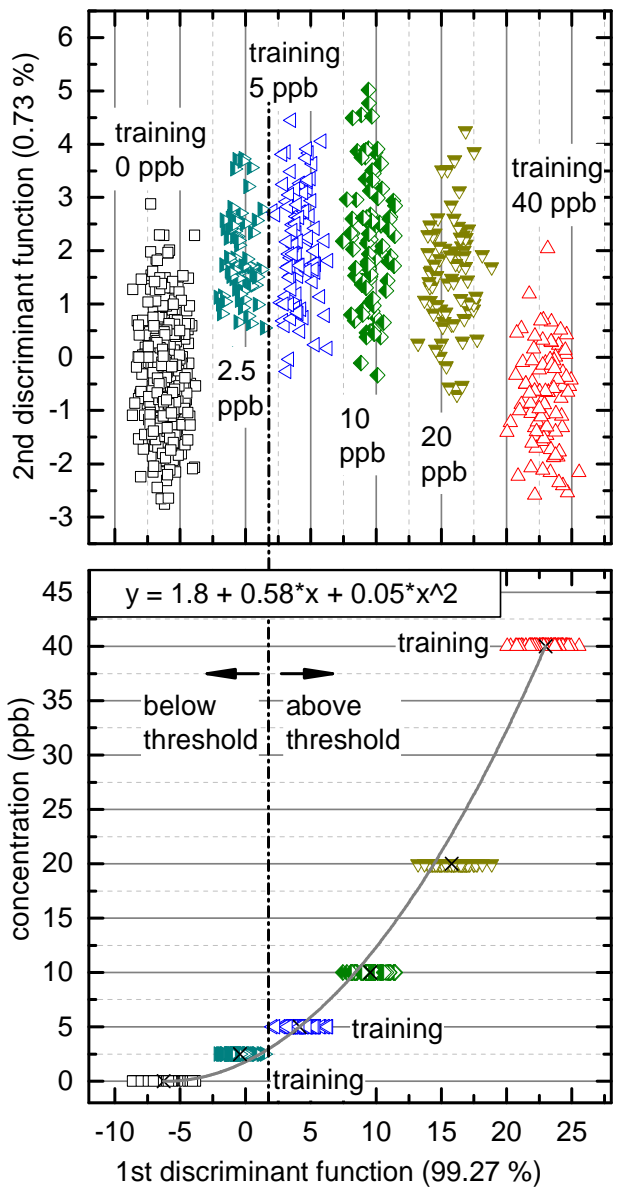

Fig. 8.24 Top: Quantification of naphthalene. Training data are marked with open symbols, projected unknown data are marked by semi-solid symbols. Bottom: Plot concentration vs. DF1 value with second order fit of the training data. The projected data are marked by semi-solid symbols. A cutting line is given which can be used as a threshold, e.g., for ventilation control. Adopted from [298], [299].

Linear Discriminant Analysis is a suitable algorithm for discrimination of different gases but not optimal for quantification purposes since it can handle only discrete groups, here concentrations, and does not offer continuous prediction. Using polynomial fit of the group centroids can cause instabilities. However, more suitable for quantification are regression algorithms which also belong to the class of 
supervised learning algorithms but are designed intentionally for determination of analog (continuous) output.

In Fig. 8.25a the regression plot of different concentrations of naphthalene is given when Partial Least Squares Regression, PLSR, is used. For building the regression model only the first eight out of 18 possible components are used. The selection has been made based on the minimum Mean Squared Error, MSR. Regression of naphthalene is possible with a standard deviation, i.e., $3 \cdot \sigma_{\max }$, of about $7 \mathrm{ppb}$. However, the centroids of each group are not located perfectly on the regression line which indicates that the linear regression model is not able to represent the data correctly over this large concentration range. In order to improve the result, the concentration range can be reduced or non-linear models, like Support Vector Regression, SVR, could be used instead. The latter one, however, requires a sufficiently larger computational power which might not be available in stand-alone VOC-monitoring systems to be installed in, e.g., private households. Although, the PLSR model is maybe not optimal, it allows a prediction of naphthalene concentrations, i.e., quantification.
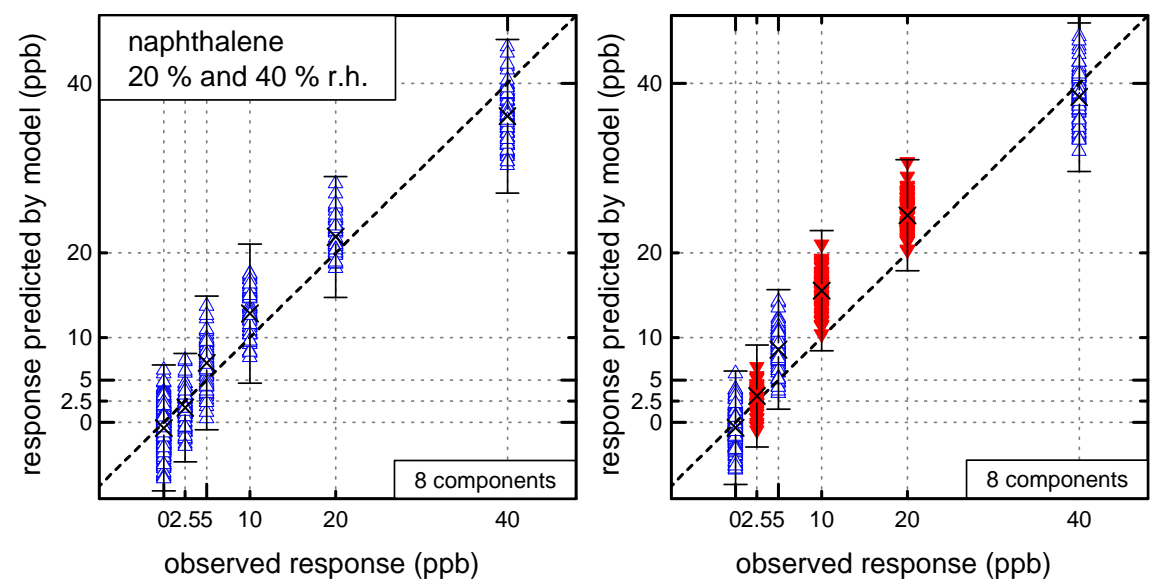

Fig. 8.25 Result of PLSR for quantification of naphthalene independent of the humidity. (a) When all concentrations are used to build the model, and (b) when only three concentrations are used. Training data are marked by blue open symbols while projected data are marked by red semi-solid symbols. Adopted from [299].

In Fig. 8.25b the regression model is built only with three concentrations, i.e., 0, 5, and $40 \mathrm{ppb}$. The left-out concentrations are projected based on the previously 
obtained regression model. Training data are marked with blue open symbols while projected data are marked by red semi-solid symbols. The standard deviation is again in the order of $7 \mathrm{ppb}$ which is sufficiently good for the given application.

\subsubsection{Detection of VOC in High Background of Ethanol}

In the previous sections, the discrimination and quantification of target VOCs in pure synthetic air is studied. For real applications, also detection of VOCs in a background of interfering gases needs to be investigated. Typically, the concentrations of interferents are a few magnitudes higher than concentrations of VOCs which need to be detected. As a typical interfering gas ethanol was chosen with concentrations of $0.5 \mathrm{ppm}$ and $1 \mathrm{ppm}$.

Fig. 8.26a shows the discrimination of formaldehyde (50, 75, 100, and $200 \mathrm{ppb}$ ), naphthalene $(5,10,20$, and $40 \mathrm{ppb})$, and benzene $(4.5,3.5,2.5$, and $1.5 \mathrm{ppb})$ in backgrounds with three different ethanol concentrations $(0,0.5$, and $1 \mathrm{ppm})$. It should be noted that benzene cannot be detected in a background of $1 \mathrm{ppm}$ ethanol and thus, this benzene group is not included in the shown discrimination. The scatter plot in Fig. 8.26a shows mainly a discrimination of the different ethanol concentrations rather than a separation of the different VOCs.
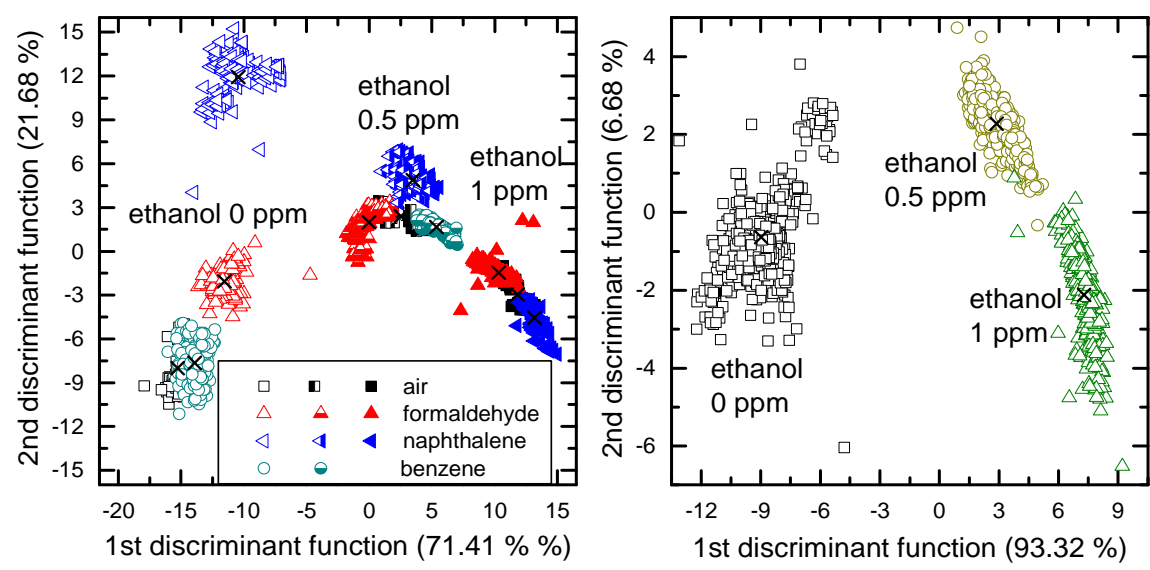

Fig. 8.26 (a) LDA scatter plot showing the discrimination of formaldehyde, naphthalene, and benzene with four concentrations each in three different ethanol backgrounds, and (b) discrimination of the three ethanol backgrounds. The level of r.h. is $20 \%$. 
Without ethanol, discrimination of formaldehyde, naphthalene, and benzene is possible, although the benzene groups overlap a lot with the pure air group. Fig. 8.26a also indicates that the discrimination of the target VOC becomes more challenging with increasing concentration of the inferring gas. The strong influence of ethanol is pointed out in Fig. 8.26b which shows the LDA scatter plot of the discrimination of the three background mixtures. Each group contains data of formaldehyde, naphthalene, and benzene with four concentrations each, except of benzene in $1 \mathrm{ppm}$ ethanol which was not possible to detect. The distinct separation of the three background groups suggests a hierarchical approach similar as described in Section 8.1.5, in which the background, i.e., the ethanol level, is determined first, followed by the discrimination of the different target gases. The second decision in a hierarchical strategy is the determination of the main pollutant. Fig. 8.27a shows the discrimination of formaldehyde, naphthalene, and benzene with four concentrations each in $0.5 \mathrm{ppm}$ ethanol. The three VOCs are nicely separated but the background group forms two clusters and one of them overlaps considerably with formaldehyde. This is an indication that detection of formaldehyde in a high background of ethanol is challenging. Fig. 8.27b shows the discrimination in $1 \mathrm{ppm}$ ethanol. It should be noted that benzene cannot be detected anymore and is thus not contained in the discrimination.
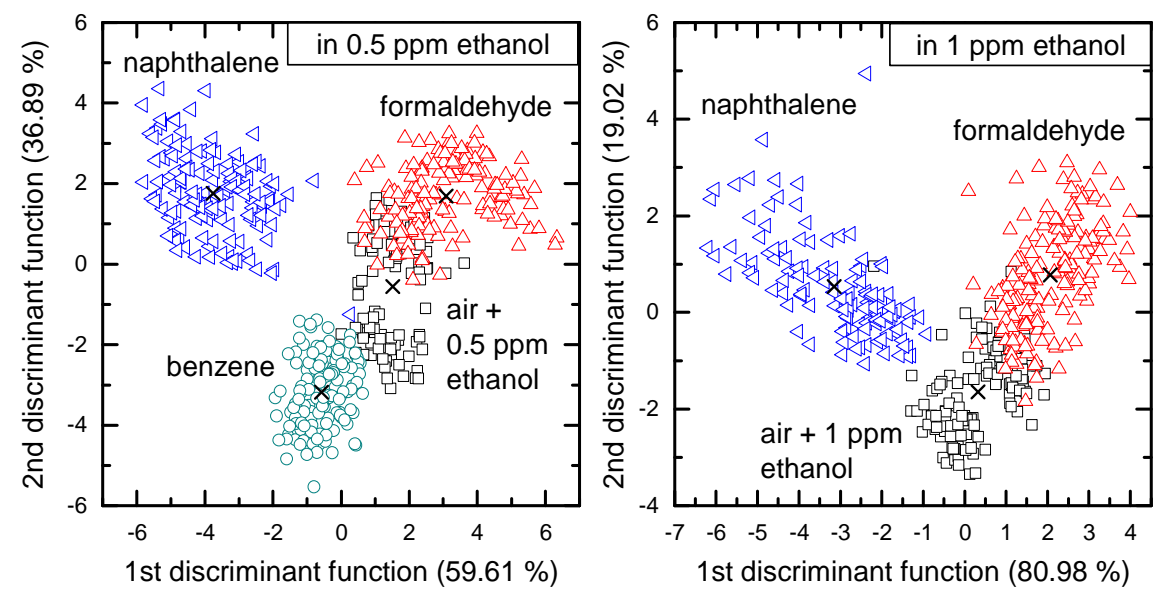

Fig. 8.27 (a) LDA scatter plot showing the discrimination of formaldehyde, naphthalene, and benzene with four concentrations each in $0.5 \mathrm{ppm}$ ethanol, and (b) discrimination of formaldehyde and naphthalene in $1 \mathrm{ppm}$ ethanol. The level of r.h. is $20 \%$. 
Besides the discrimination of the three chosen VOCs, quantification is of great interest. In Fig. 8.28a the discrimination of different concentrations of naphthalene in backgrounds with $0,0.5$, and $1 \mathrm{ppm}$ ethanol is given. It can be observed that the discrimination mainly separates between ethanol and no-ethanol. Determination of the naphthalene concentration without ethanol in the background is easily possible whereas the concentration-groups with ethanol strongly overlap.
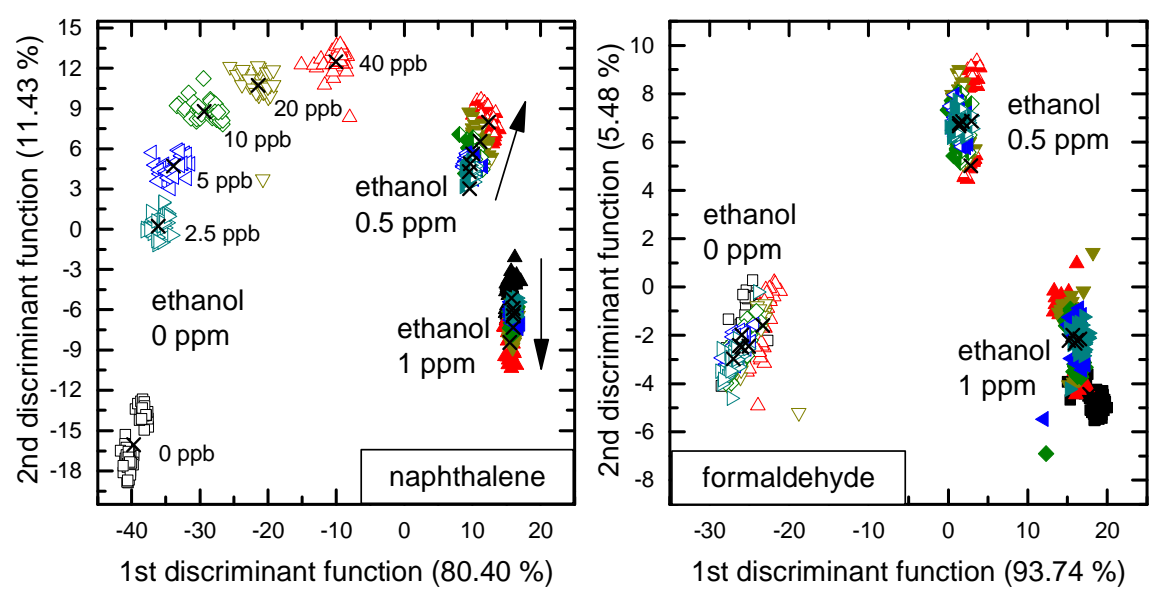

Fig. 8.28 (a) LDA scatter plot showing the discrimination of different naphthalene concentrations in three different ethanol backgrounds, and (b) discrimination of different formaldehyde concentrations. The level of r.h. is $20 \%$.

Also in case of formaldehyde, cf. Fig. 8.28b, the discrimination mainly separates the level of ethanol rather than the formaldehyde concentrations. Even the determination of the concentration without ethanol in the background is not possible. In general, detection and quantification of naphthalene is easier than formaldehyde. Very challenging is benzene which might be because the concentrations of interest are ten times lower than for naphthalene.

In Fig. 8.28a a distinct separation between no ethanol and high concentrations of ethanol in the background can be observed. In addition to that, for the LDA calculation given in Fig. 8.29 data from $0.5 \mathrm{ppm}$ and $1 \mathrm{ppm}$ ethanol are combined in order to discriminate different naphthalene concentrations. The scatter of the groups is high but at least 10, 20, and 40 ppb are separable. 


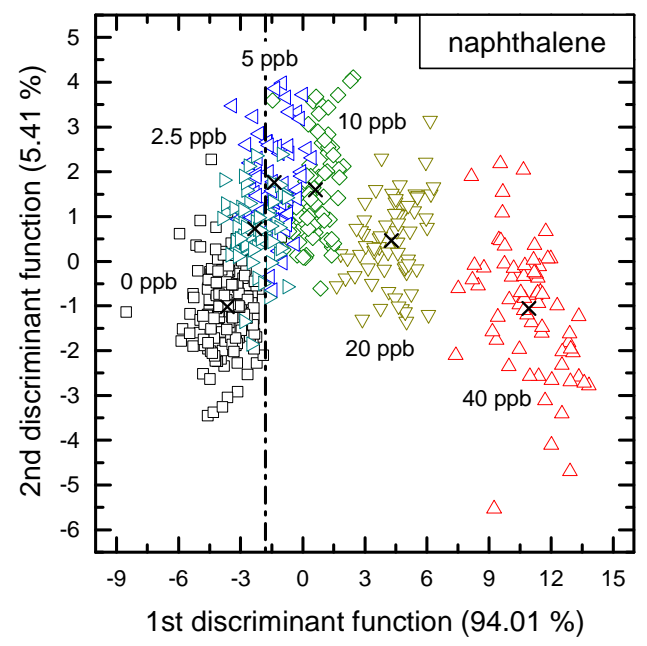

Fig. 8.29 LDA scatter plot showing the discrimination of different naphthalene concentrations. Each group contains data with two different background ethanol concentrations, i.e., $0.5 \mathrm{ppm}$ and $1 \mathrm{ppm}$. The level of r.h. is $20 \%$.

The overall cross-validation rate of LOOCV with a Mahalanobis distance classifier is almost $87 \%$. For the 10, 20, and $40 \mathrm{ppb}$ groups, the rates are $91 \%, 100 \%$, and $100 \%$, respectively, cf. Table 11 . Remembering that the threshold value for naphthalene is approximately $5 \mathrm{ppb}$, determination of the concentrations being interesting for on demand ventilation control is possible. The threshold is marked with the dashed line in Fig. 8.29.

Table 11 Leave-one-out cross-validation of the discrimination of different naphthalene concentrations in ethanol, cf. Fig. 8.29, using a Mahalanobis distance classifier.

\begin{tabular}{lr}
\hline group & classification rate \\
\hline overall & $86.9 \%$ \\
0 ppb naphthalene & $90.3 \%$ \\
$\mathbf{2 . 5}$ ppb naphthalene & $67.2 \%$ \\
$\mathbf{5}$ ppb naphthalene & $63.1 \%$ \\
$\mathbf{1 0}$ ppb naphthalene & $91.3 \%$ \\
$\mathbf{2 0}$ ppb naphthalene & $100 \%$ \\
$\mathbf{4 0}$ ppb naphthalene & $100 \%$ \\
\hline
\end{tabular}




\subsubsection{Conclusion Detection of Volatile Organic Compounds}

In this sub-chapter, silicon carbide field effect transistors were studied for indoor air quality application. Since this was the first time that this kind of sensor has been used to detect volatile organic compounds, a rather simple temperature cycle was chosen. For platinum gate SiC-FETs, the cycle consists of three temperature plateaus, i.e., $180{ }^{\circ} \mathrm{C}, 200{ }^{\circ} \mathrm{C}$, and $220{ }^{\circ} \mathrm{C}$. Due to the used read-out electronics, it is necessary to operate the sensor in the linear region where the temperature range is limited. Nevertheless, the sensor shows a sufficiently high relative sensor response to formaldehyde, naphthalene, and benzene in the relevant concentration range. The highest sensor response is achieved for naphthalene and the influence of relative humidity is in general small. Discrimination of the three target volatile organic compounds is possible when using Linear Discriminant Analysis. In order to control a ventilation system, it is important to detect whenever one of the target gases exceeds its guideline value. Thus, the suggested approach consists of a "below threshold" group which combines data from pure air and all target gases with concentrations below their guideline value. In addition to that, Partial Least Square Discriminant Analysis, was studied since it does not only transform the data but at the same time also does classification. The quality of the discrimination is slightly better for LDA but since the classification threshold in PLS-DA is easily adjustable, PLS-DA is a promising algorithm for this application.

Quantification of target VOCs independent of the level of relative humidity is possible with LDA and Partial Least Squares Regression. For a rough estimation of the concentration LDA together with a polynomial fit function is sufficient but if continuous prediction is needed, PLSR might outperform LDA since regression models are explicitly designed for (continuous) concentration prediction.

Discrimination and quantification of VOCs in a high background of ethanol is also possible. Still, ethanol affects the sensor response considerably and the influence on the discrimination is higher than the influence of the target VOCs. Thus, a hierarchical strategy can be used to determine the background first. However, the sensor was not able to detect benzene in a background with $1 \mathrm{ppm}$ ethanol. Also, a change in humidity and in the ethanol concentration at the same time will be challenging to compensate. 


\subsection{Combination of Temperature and Gate- Bias Cycling}

In Chapter 7, it was shown that temperature cycled operation, TCO, as well as gate bias cycled operation, GBCO, are sufficient methods to increase the selectivity of SiC-FETs. Since the sensor needs an elevated temperature to operate, there is naturally a link between temperature and gate bias cycling. In Section 7.3.4, the combination of a temperature and gate bias cycling is indicated. In this subchapter, the methodology of the combination of temperature and gate bias cycled operation is further developed, cf. Section 8.3.1. In order to demonstrate the arising capabilities, results dealing with quantification, cf. Section 8.3.2, and discrimination of exhaust gases, Section 8.3.3, are presented. Discrimination of principal components is studied as an alternative feature extraction and feature transformation technique in Section 8.3.4. Section 8.3.5 and Section 8.3.6 deal with the stability of the suggested approach and drift compensation. The last section summarizes the main finding achieved. This sub-chapter is based on [301], [219].

\subsubsection{Methodology and Used Cycle}

In this study, three-terminal depletion type $\mathrm{SiC}$-FET sensors with a porous platinum gate as described in Section 6.2.3 are used. In order to allow cycling the temperature and the applied gate bias over a wide range, the sensor is operated at a constant drain-source voltage, $V_{D S}=4 \mathrm{~V}$, i.e., in saturation, see also Section 7.1.2. The suggested combined temperature and gate-bias cycle is shown in Fig. 8.30a. For introducing this new method, a rather simple cycle consisting of two temperature plateaus, i.e., $260{ }^{\circ} \mathrm{C}$ and $200{ }^{\circ} \mathrm{C}$ respectively, each of them lasts for 45 s, i.e., the total length of the combined cycle is $90 \mathrm{~s}$. 


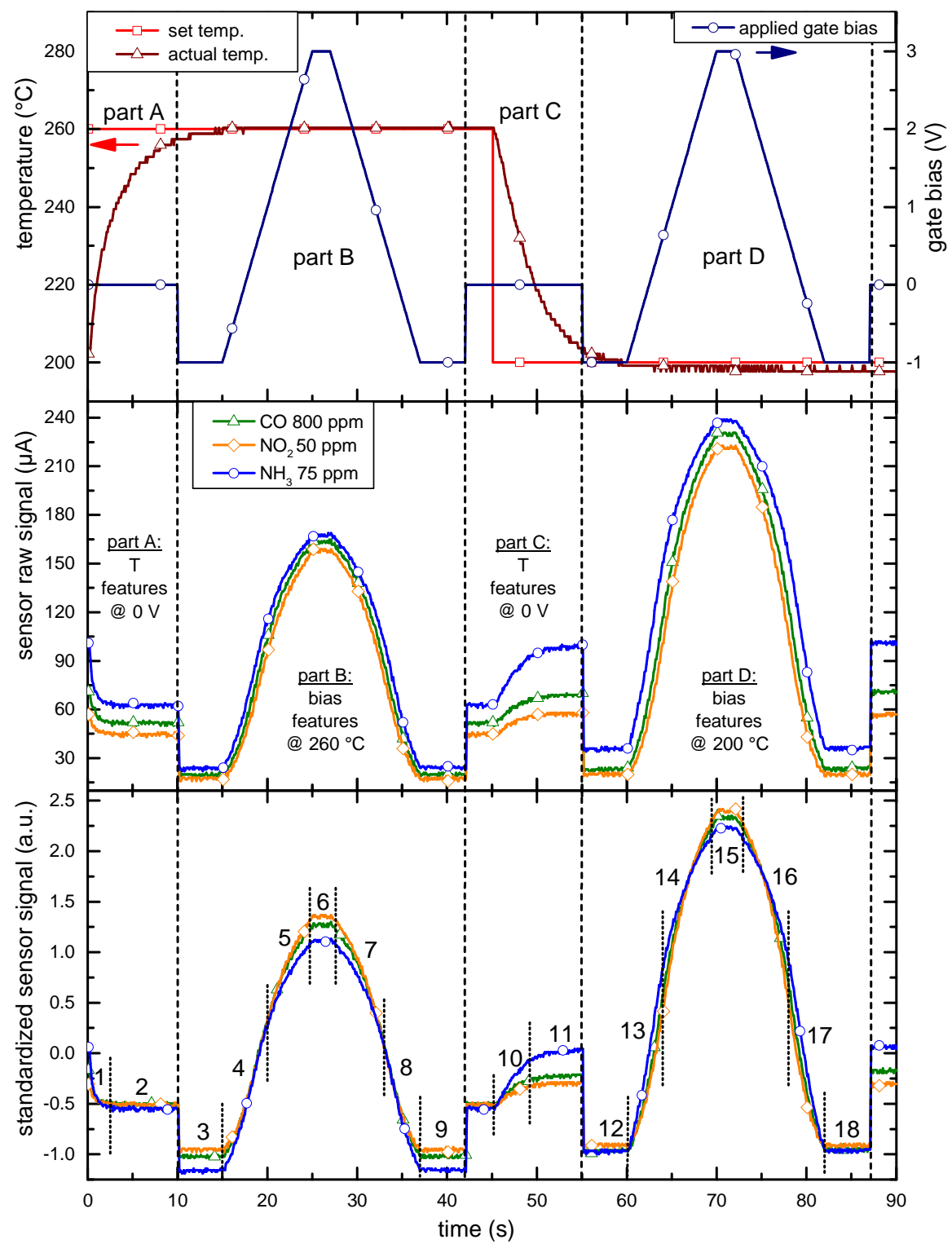

Fig. $8.30 \quad$ (a) Combination of temperature cycle (part A and C) consisting of two plateaus and gate bias cycle combining plateaus and ramps (parts B and D), (b) dynamic sensor response for $800 \mathrm{ppm} \mathrm{CO}, 50 \mathrm{ppm} \mathrm{NO}_{2}$, and $75 \mathrm{ppm} \mathrm{NH}_{3}$ in $\mathrm{N}_{2}$ with $5 \% \mathrm{O}_{2}$, and (c) standardized dynamic sensor response with marked feature intervals. Adopted from [219]. 
During temperature change the applied gate bias is zero in order to not mix different effects. On each temperature plateau the applied gate bias is modulated after the temperature $^{36}$ is stabilized. After $10 \mathrm{~s}$ the gate bias is set to $-1 \mathrm{~V}$ for $5 \mathrm{~s}$ and then ramped to $+3 \mathrm{~V}$ in $10 \mathrm{~s}$, i.e., with a slope of $0.4 \mathrm{~V} / \mathrm{s}$. After $2 \mathrm{~s}$, the bias is decreased with the same slope to $-1 \mathrm{~V}$ again. The bias is kept constant for $5 \mathrm{~s}$ and then set to $0 \mathrm{~V}$ for $3 \mathrm{~s}$, then the temperature is lowered from $260{ }^{\circ} \mathrm{C}$ to $200{ }^{\circ} \mathrm{C}$ before the next gate bias cycle starts.

Hence, the temperature cycle can be seen as the outer cycle and the gate bias cycle as the inner cycle. The combined cycle can be divided into four main parts, cf. Fig. 8.30b: part $\mathrm{A}$ and $\mathrm{C}$ where the temperature changes while the applied bias is constant and set to $0 \mathrm{~V}$, and part $\mathrm{B}$ and $\mathrm{C}$ where the temperature is constant but the bias changes. This allows studying both operating modes nearly independently. The sensor response follows the temperature as well as the applied bias and is altered differently for different gases, e.g., $\mathrm{CO}, \mathrm{NO}_{2}$, and $\mathrm{NH}_{3}$ in dry nitrogen with $5 \%$ oxygen, as shown in Fig. 8.30b. In order to reduce the influence of sensor drift and different scaling of the cycles, the sensor response, cf. Fig. 8.30, has been standardized cycle-wise, cf. Eq. (5.6), i.e., the cycle mean is set to 0 and the standard deviation to 1 . In static operation, this kind of pre-processing is sometimes called sensor auto-scaling [150]. The standardized response is given in Fig. 8.30c. In total, 18 intervals are defined in which shape-describing features, like mean values and slopes were extracted, in the following called standard features. Features calculated in intervals 1,2, and 10, 11 represent the sensor response due to temperature, and features from intervals 3-9 as well as 12-18 describes the sensor response due to a change in gate bias. These 36 features are the basis for pattern recognition, in particular Linear Discriminant Analysis, LDA.

\subsubsection{Quantification of Carbon Monoxide}

The influence of the different features on the discrimination is exemplarily studied for the quantification of carbon monoxide, i.e., 200, 400, 600, and $800 \mathrm{ppm}$. In a first step, only features from part $\mathrm{A}$ and $\mathrm{C}$, cf. Fig. 8.30, i.e., features representing the

\footnotetext{
${ }^{36}$ The actual temperature of the sensor was determined by a Pt-100 temperature sensor which is glued next to the SiC chip on the ceramic heater as described in Section 6.2. The temperature on the sensor surface can vary from the measured one.
} 
temperature change are used for the quantification of $\mathrm{CO}$ by LDA. The resulting LDA scatter plot is shown in Fig. 8.31a. The groups corresponding to different $\mathrm{CO}$ concentrations are separated with a correct classification rate of leave-one-out crossvalidation with a Mahalanobis distance classifier of $99 \%$.

In a next step, only features from the gate bias change at $200{ }^{\circ} \mathrm{C}$, i.e., part $\mathrm{D}$ of the combined cycle, cf. Fig. 8.30, are used. Similar results as before in terms of class separation can be achieved, cf. Fig. 8.31a and b. However, when using features from all parts of the combined cycle, the quality of the discrimination of the different concentrations of $\mathrm{CO}$ can be enhanced. Especially, the separation of the two highest concentrations, i.e., $600 \mathrm{ppm}$ and $800 \mathrm{ppm}$, is significantly better. Nevertheless, the number of features is increased which might be undesirable in certain applications.
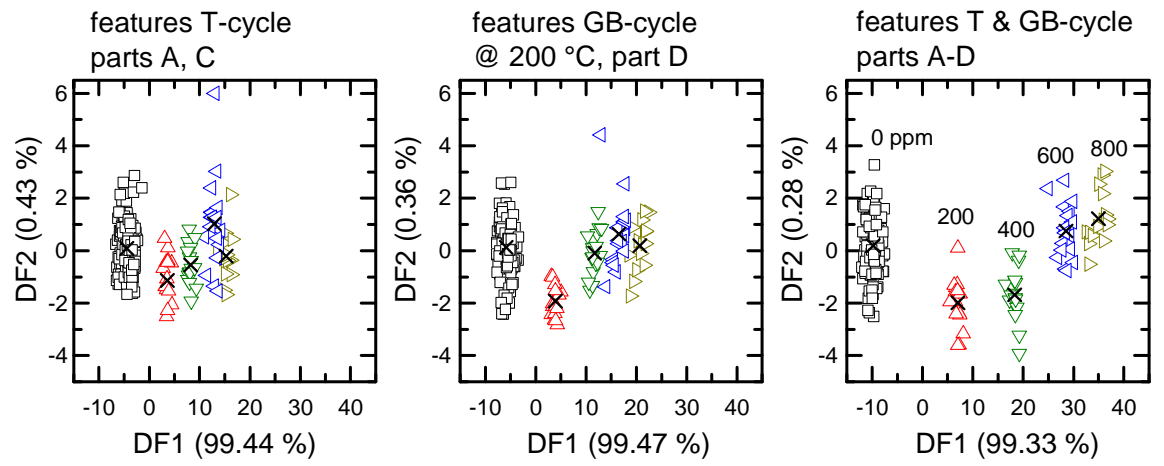

Fig. 8.31 LDA scatter plots showing the quantification of carbon monoxide when using features from (a) only the temperature change, i.e., parts A and C, (b) only from the bias cycle at $200{ }^{\circ} \mathrm{C}$, i.e., part $\mathrm{D}$, and (c) the temperature change and both gate bias cycles. Adopted from [219].

The quality of the discrimination can be expressed by several parameters, cf. Section 5.3 and Section 5.5. Probably the most significant parameter is the correct cross-validation rate of, e.g., Leave-one-out cross-validation, LOOCV, since it describes the discrimination itself. In contrast to that, there are also statistical parameters, like the multivariate Wilks' Lambda $\Lambda_{\text {multi }}$ and the cluster separability $J$, which determines the quality prior to the discrimination, i.e., based on the within and between groups sums of squares matrixes $\boldsymbol{B}$ and $\boldsymbol{W}$. However, to relatively compare 
LDA scatters plot, all three of them can be used. For the discrimination shown in Fig. 8.31, these parameters are summarized in Table 12 .

Table 12 Parameters describing the quality of the discrimination shown in Fig. 8.31.

\begin{tabular}{lrrr}
\hline & $\begin{array}{r}\text { features T-cycle } \\
\text { parts A, C }\end{array}$ & $\begin{array}{r}\text { features GB-cycle } \\
\text { @ 200 }\end{array}$ & $\begin{array}{r}\text { features T } \mathbf{C} \text {, part D } \\
\text { parts A-D }\end{array}$ \\
\hline LOOCV & $99.07 \%$ & $98.14 \%$ & $99.53 \%$ \\
Wilks' Lambda $\Lambda_{\text {multi }}$ & 0.016 & 0.008 & 0.002 \\
cluster separability J & $94.13 \%$ & $95.17 \%$ & $95.25 \%$ \\
\hline
\end{tabular}

It can be observed that cross-validation rate is the highest when features from all parts of the combined cycle are used. Also the two statistical parameters substantiate this finding. All three LDA allow determination of the $\mathrm{CO}$ concentration by the first discriminant function which holds over $99 \%$ of the discriminatory information. The second function represents basically noise and can be neglected.

\subsubsection{Discrimination and Feature Selection}

As indicated in the previous section, using features from all parts of the combined cycle improves the quality of quantification of $\mathrm{CO}$. However, at the same time the number of features is highly increased which suggests feature (pre-)selection. There are several ways of selecting the most significant features as discussed in Section 7.4.1. At this point, the change of the feature values over the measurement, i.e., time is considered first and can be seen as feature pre-selection.

For discrimination purposes, e.g., the discrimination of $\mathrm{CO}, \mathrm{NO}_{2}$, and $\mathrm{NH}_{3}$, features which change their value significantly for different gas exposures, carry the required information and are thus, suitable for discrimination. If they are indeed significant for the discrimination cannot be stated here but at least they look promising. Fig. 8.32 shows exemplarily some mean values, mv, and their change due to exposure of test gases and different concentrations. In the test gas profile, there are breaks between the different concentrations, i.e., just carrier gas was applied, to avoid memory effects and accumulative behavior of the sensor. These breaks are not 
shown in Fig. 8.32. For instance, feature mv2 (part A of the combined cycle) shows a binary response to ammonia but does not reflect any changes in the concentration.

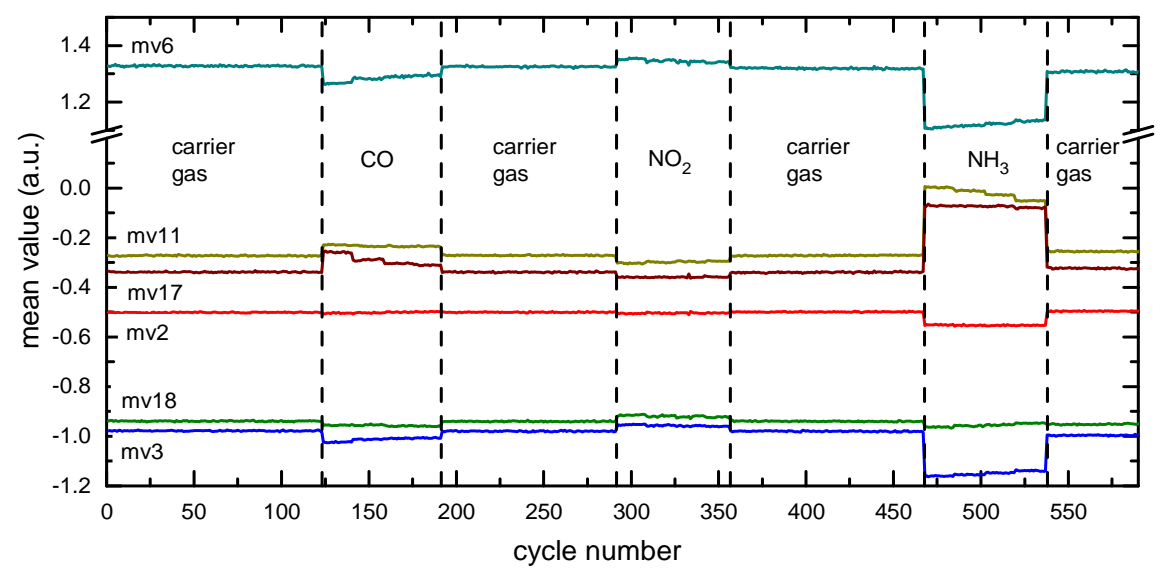

Fig. 8.32 Exemplary features (here: mean values, $\mathrm{mv}$ ) and their change over time due to exposure to different test gases and different concentrations. The carrier gas is $5 \%$ oxygen in nitrogen. Reproduced from [219] with kind permission from Elsevier.

In order to quantify $\mathrm{NH}_{3}$, feature mv11 (part C) might be used additionally which, however, also shows a response to $\mathrm{CO}$ and $\mathrm{NO}_{2}$. In a similar way, feature mv17 (part D) provides information on different concentrations of CO. Thus, to be able to discriminate three different gases with four concentrations each, it is evident that several features and, in particular features from different parts of the combined cycle, cf. Fig. 8.30, are required. The suggested strategy can easily be automated by calculating the correlation between the feature value and the gas exposure [247]. Typically, pattern recognition tools like LDA are used for evaluation. The LDA scatter plot of such a discrimination using all features is shown in Fig. 8.33a.

Discrimination of $\mathrm{CO}, \mathrm{NO}_{2}$, and $\mathrm{NH}_{3}$ independent of the concentration is easily possible with almost now overlapping of the different groups. Leave-one-out crossvalidation, LOOCV, with a Mahalanobis distance classifier leads to a correct classification rate of almost $95 \%$, only the different concentrations of $\mathrm{NO}_{2}$ overlap to some extent and have a lower validation rate. However, direct determination of the concentration of $\mathrm{CO}$ and $\mathrm{NH}_{3}$ is possible. The cluster separability is very high as well which indicates that the data set is well-conditioned and the requirements for using LDA are fulfilled. Although, feature extraction reduces the dimension of the input 
data considerably, for the discrimination shown in Fig. 8.33a 36 features are used, i.e., the dimension of the input data is 36 which is rather high.
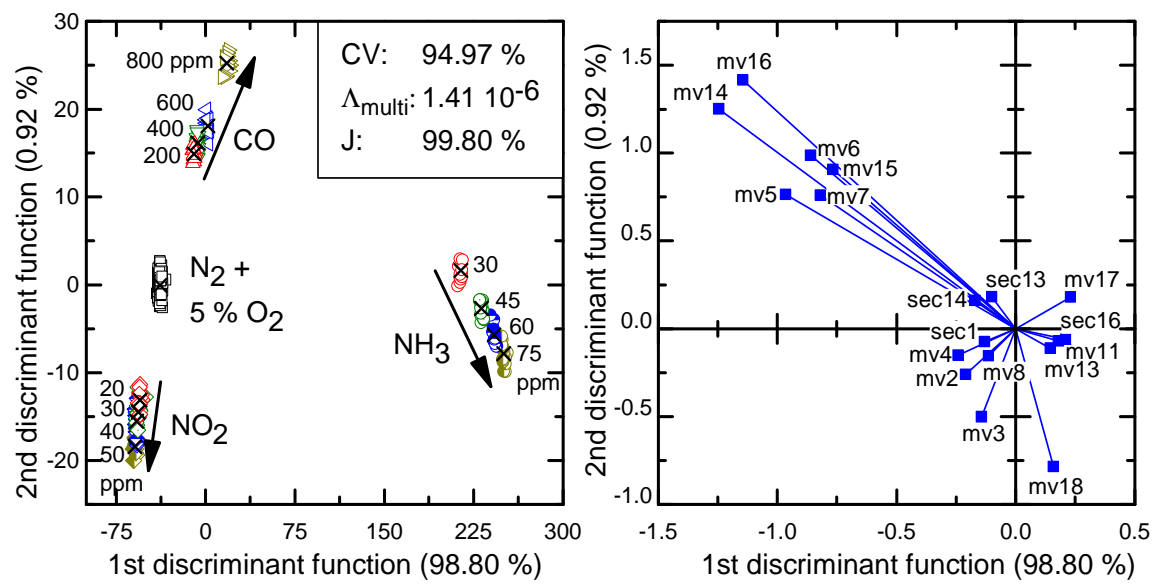

Fig. 8.33 (a) LDA showing the discrimination of $\mathrm{CO}, \mathrm{NO}_{2}$, and $\mathrm{NH}_{3}$ with four concentrations each as well as the leave-one-out cross-validation rate, $\mathrm{CV}$, the multivariate Wilks' Lambda $\Lambda_{\text {multi, }}$ and the cluster separability $J$. (b) Loadings plot (only features with an average coefficient greater than 0.1 are shown), mv: mean value, sec: secant. Reproduced from [219] with kind permission from Elsevier.

The corresponding loadings plot is given in Fig. 8.33b and can be used for feature selection after discrimination. It should be noted that in this loading plot only features with an average coefficient greater than 0.1 are shown since any feature located close to the origin does not contribute to the discrimination significantly. The loadings plot is in principle ${ }^{37}$ the graphical representation of the average coefficient, cf. Eq. (5.26).

The value of the average coefficient of the first 18 most significant features, i.e., with a value greater than 0.1 , are shown in Fig. 8.34 which can be easily used for feature selection. Nevertheless, the loadings plot provides additional, important information regarding each feature. The direction of the feature loadings together with the scatter plot provides information which feature contributes in which way to the discrimination. This information is not contained in the average coefficient.

\footnotetext{
${ }^{37}$ The average coefficient is calculated for all theoretically possible discriminant functions whereas the loadings plot shows usually only a two dimensional representation of the feature loadings. However, since in this example the first two discriminant functions carry $99.7 \%$ of the discriminatory information, discriminant functions of higher order can be neglected.
} 


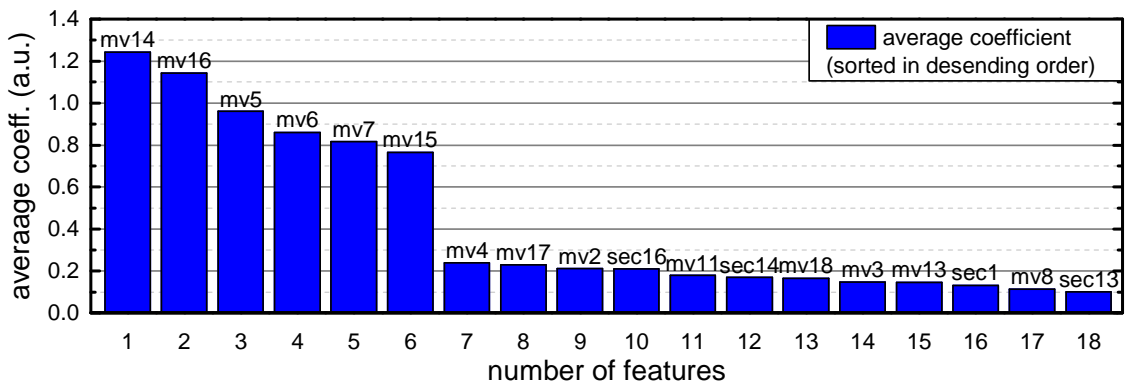

Fig. 8.34 Diagram showing the value of the average coefficient for the 18 most significant features, i.e., features with an average coefficient greater than 0.1 .

Feature selection is typically done based on the average coefficient first in order to pick only the significant features. There is no threshold value for the selection but it is rather based on the user's experience and on the maximum wanted dimension of the input data. Fig. 8.35a shows the LDA scatter plot when only the 18 features with an average coefficient greater than 0.1, cf. Fig. 8.34, were used. Fig. 8.34 indicates that only six features are significant but initially one should not exclude too many features at once. Taking the first 18 features still means that the dimension is halved.
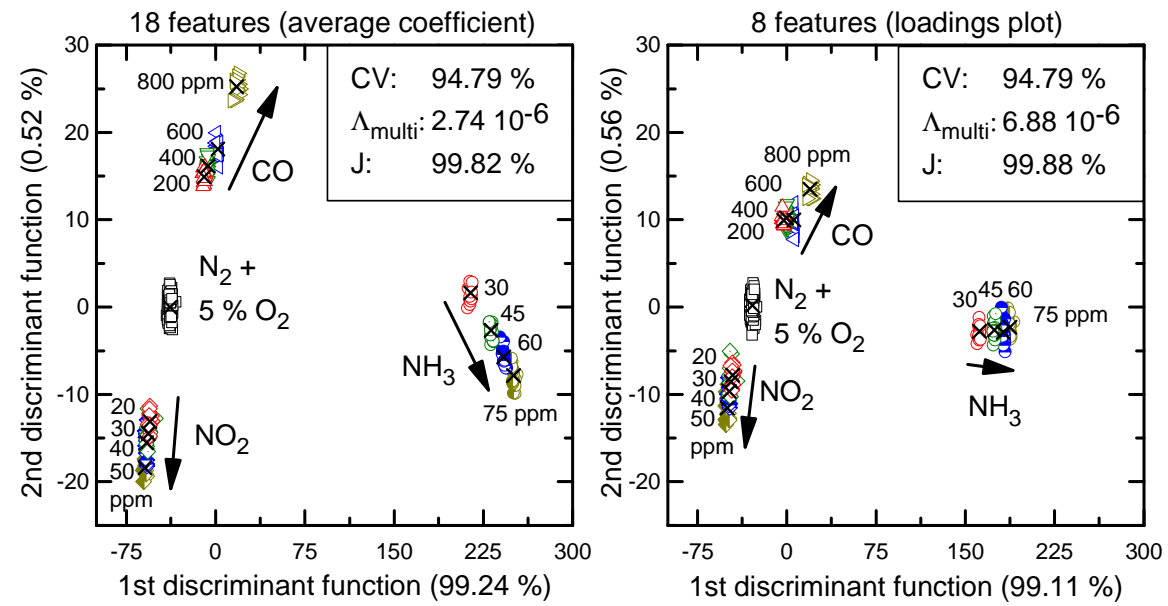

Fig. 8.35 (a) LDA showing the discrimination of $\mathrm{CO}, \mathrm{NO}_{2}$, and $\mathrm{NH}_{3}$ with four concentrations each when only features with an average coefficient greater than 0.1 were used as well as the leave-one-out cross-validation rate, $\mathrm{CV}$, the multivariate Wilks' Lambda $\Lambda_{\text {multi }}$ and the cluster separability $J$. (b) Feature selection based on the loadings plot. In total eight features were used. 
The discrimination is as good as before when comparing the scatter plots in Fig. 8.33a and Fig. 8.35a as well as the validation rates and cluster separability. As mentioned earlier, the loadings plot additionally can be used to exclude redundant features. Redundant features have loadings pointing in the same direction.

By looking at the loadings plot in Fig. 8.33b which shows the 18 selected features, there are some with loadings pointing in similar directions. For instance mv6 and mv15 point in the same direction as mv16. Since mv16 is located further apart from the origin, i.e., has a greater average coefficient value, is should be kept while the others can be left out. Hence, from the 18 features after selection by the loadings plot, eight features are kept, cf. Table 13.

Table 13 Features selected by the average coefficient and the loadings plot.

\begin{tabular}{lll}
\hline feature & $\begin{array}{l}\text { average } \\
\text { coefficient }\end{array}$ & description of their location in the cycle \\
\hline $\mathbf{m v 1 4}$ & 1.24 & Part D, bias ramp from $+1 \mathrm{~V}$ to $+3 \mathrm{~V}$ at $200{ }^{\circ} \mathrm{C}$ \\
$\mathbf{m v 1 6}$ & 1.14 & Part D, bias ramp from $+3 \mathrm{~V}$ to $+1 \mathrm{~V}$ at $200{ }^{\circ} \mathrm{C}$ \\
$\mathbf{m v 4}$ & 0.86 & Part B, bias ramp from $-1 \mathrm{~V}$ to $+1 \mathrm{~V}$ at $260{ }^{\circ} \mathrm{C}$ \\
$\mathbf{m v 1 7}$ & 0.23 & Part D, bias ramp from $+1 \mathrm{~V}$ to $-1 \mathrm{~V}$ at $200{ }^{\circ} \mathrm{C}$ \\
$\mathbf{s e c 1 6}$ & 0.21 & Part D, bias ramp from $+3 \mathrm{~V}$ to $+1 \mathrm{~V}$ at $200{ }^{\circ} \mathrm{C}$ \\
$\mathbf{m v 1 1}$ & 0.18 & Part C, temperature plateau at $200{ }^{\circ} \mathrm{C}$, zero bias \\
$\mathbf{m v 1 8}$ & 0.16 & Part D, bias plateau at $-1 \mathrm{~V}$ at $200{ }^{\circ} \mathrm{C}$ \\
$\mathbf{m v 3}$ & 0.15 & Part C, bias plateau at $-1 \mathrm{~V}$ at $260{ }^{\circ} \mathrm{C}$ \\
\hline
\end{tabular}

The corresponding LDA scatter plot when only these eight features are used is shown in Fig. 8.35b. The groups move closer to each other but still the separation is as good as before when looking at the validation rate. The cluster separability is slightly improved while the value of Wilks' Lambda is higher than before which basically means that the groups are getting closer to each other. However, the discrimination and determination of the different concentrations of $\mathrm{CO}$ and $\mathrm{NH}_{3}$ is easily possible. It can be observed that features from different bias regions and temperatures are selected which again proved the methodology of combining temperature and gate bias cycled operation. In Fig. 8.36 a detailed view of the discrimination shown in Fig. 8.35b for the different concentrations of the three gases is given. 

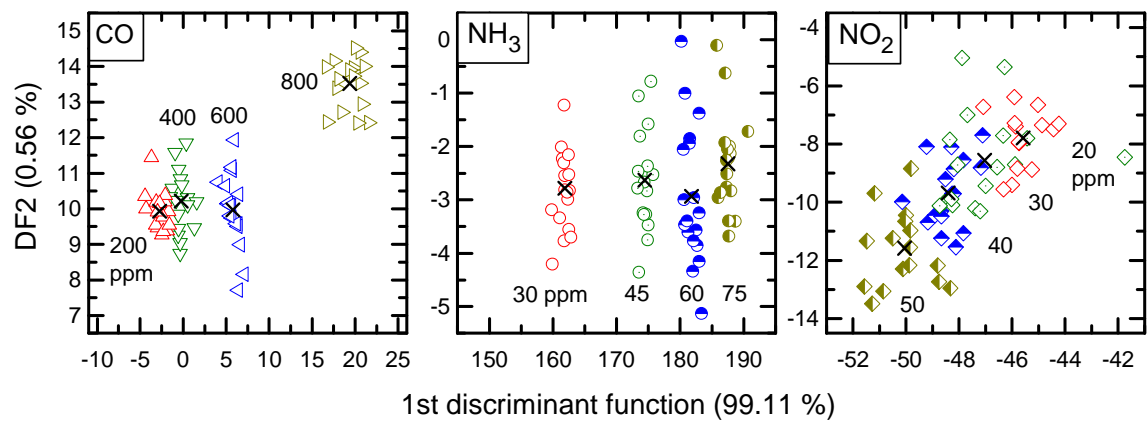

Fig. 8.36 Detailed view of the discrimination shown in Fig. 8.35b. (a) For carbon monoxide, (b) for ammonia, and (c) for nitrogen dioxide.

The scatter plot shown in Fig. 8.35b suggests that the groups corresponding to different concentrations strongly overlap, but this is just a scaling effect. The detailed view, cf. Fig. 8.36, shows that a direct determination of the concentration of $\mathrm{CO}$ and $\mathrm{NH}_{3}$ is possible, and to some extent also for $\mathrm{NO}_{2}$.

\subsubsection{Feature Transformation by Principal Component Analysis}

Besides the extraction of standard features, i.e., mean values and slopes, which are typically used in the virtual multi-sensor approach, Principal Component Analysis can be applied instead as mentioned earlier in Section 5.2.1. Using PCA for feature extraction has the advantage that all calculated principal components, PCs, i.e., features, are orthogonal and thus linear independent.

This is in particular interesting if either extraction of standard features is difficult, the number of extracted features is high, or if the extracted features are correlated, i.e., not linear independent. Thus, there are basically two options to apply discriminant analysis of principal components: First, the sensor raw data, i.e., each data point in a cycle is used as input for the PCA. Second, standard features are extracted first and are then used as input for PCA. After that, the calculated PCs, or a selection of those, are used as features for the Linear Discriminant Analysis.

In order to show the capability of this strategy, the same data set as used in the previous sections is considered again. First, the sensor raw data are used as input for 
PCA. Fig. 8.37 shows the PCA scatter plot showing the discrimination of $\mathrm{CO}, \mathrm{NO}_{2}$, and $\mathrm{NH}_{3}$ with four concentrations each. Already this projection leads to a good separation of the different groups and using LDA in a second step is maybe not necessary. However, in this section the suitability of the method itself for dynamically operated SiC-FETs is studied rather than an optimization to a specific application. The data set used for the discrimination in Fig. 8.37 contains 536 cycles and each cycle consists of 900 data points. Thus, 536 different principal components can theoretically be calculated.

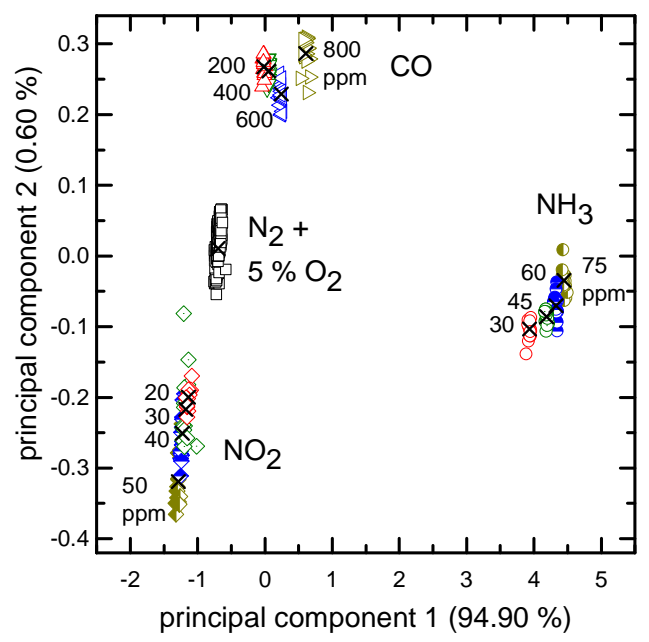

Fig. 8.37 PCA scatter plot showing the discrimination of $\mathrm{CO}, \mathrm{NO}_{2}$, and $\mathrm{NH}_{3}$ with four concentrations each when the sensor data, i.e., all 900 data points per cycle, is used as input for calculating the PCA.

However, to cover, e.g., $95 \%$ of the variance contained in the data, it is enough to take the first two PCs, cf. Fig. 8.37. In the second step, i.e., the LDA, the first 36 PCs are taken in order to have the same dimension as it was the case for the standard features, cf. Section 8.3.3.

This allows a direct comparison of the two feature extraction methods. The resulting LDA scatter plot when the first 36 PCs are used is given in Fig. 8.38a. The quality of the discrimination is comparable to Fig. 8.33 where the 36 standard features are used as input for the LDA. The cross-validation, CV, rate when using LOOCV with a Mahalanobis distance classifier is for both $94.97 \%$. The values of the cluster 
separability $J$ and the multivariate Wilks' Lambda are slightly better in case of the standard features. The loadings plot for the discriminant analysis of principal components is shown in Fig. 8.38b (only PCs with an average coefficient greater than 0.8 are shown). As can be seen, only seven PCs contribute significantly to the discrimination. Furthermore, it should be mentioned that these are not the first seven PCs. This is an indication that PCA as an unsupervised method does not necessarily lead to the best discrimination and, that the discriminatory information in this problem is not exclusively expressed in the variance. In discriminant analysis of principal components, LDA selects the PCs which contribute most to the discrimination.
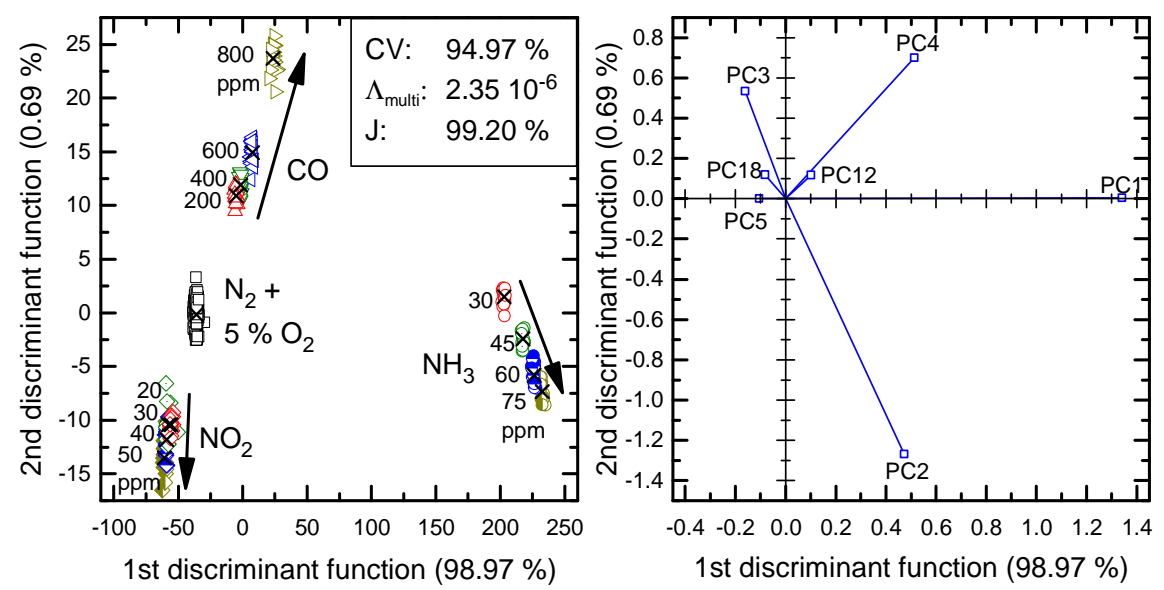

Fig. 8.38 (a) LDA scatter plot showing the discrimination of $\mathrm{CO}, \mathrm{NO}_{2}$, and $\mathrm{NH}_{3}$ with four concentrations each when the first 36 principal components are used as features. The PCA is calculated based on sensor data. (b) Corresponding loadings plot. Only features with an average coefficient greater than 0.8 are shown.

The just mentioned strategy does not lead automatically to a dimension reduction. As mentioned earlier, for the given problem 536 principal components are theoretically possible to calculate which are too many as input for a supervised method like LDA.

From the calculated PCs, a subset needs to be taken for use in LDA. Alternatively, standard features can be calculated first, e.g., mean values and slopes as done in Section 8.3.3. These 36 features are than used as input for the PCA. Since mean values and slopes are of different magnitude, it is important to use standardized PCA 
in order to ensure equal weighting of each feature. The resulting PCA scatter and loadings plot are shown in Fig. 8.39.
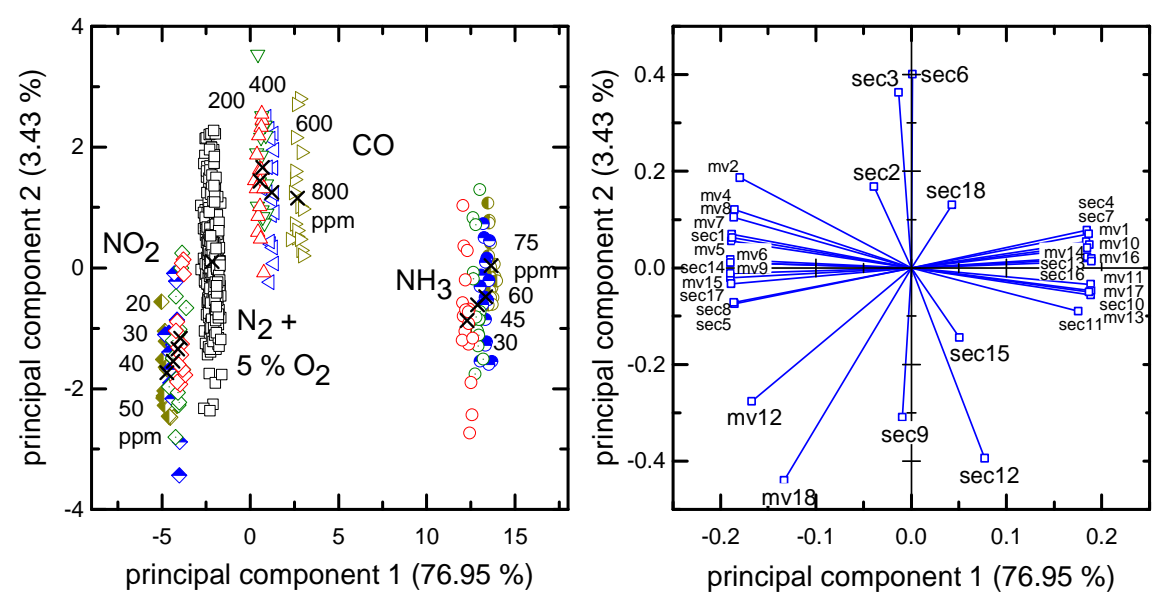

Fig. 8.39 (a) Scatter plot of a standardized PCA showing the discrimination of $\mathrm{CO}, \mathrm{NO}_{2}$, and $\mathrm{NH}_{3}$ with four concentrations each when all extracted features are used, and (b) corresponding loadings plot.

The scatter is much higher and the separation of the groups is not as good as before. The loadings plot shows that the features are weighted equally and that most of the features contribute to a separation along the first axis, i.e., PC1. In a second step, all 36 possible PCs are used for LDA. Fig. 8.40 shows the resulting LDA scatter plot. The quality of the discrimination is as good as before, cf. Fig. 8.33 and Fig. 8.38. The corresponding loadings plot again indicates that not the first PCs are the most significant features in LDA. This is because LDA does not focus on the variance in the data set as it is represented by PCA but rather on optimization of the separation of the different groups.

In summary, discriminant analysis of principal components is suitable for dynamically operated SiC-FETs. The result is comparable to the one obtained by using standard features. Since the ratio between cycles and extracted features is critical due to the tendency of LDA to over-fit data, and the fact that features need to be uncorrelated, it might be sufficient to transform the extracted features first by PCA and then use selected PCs as input for LDA. 

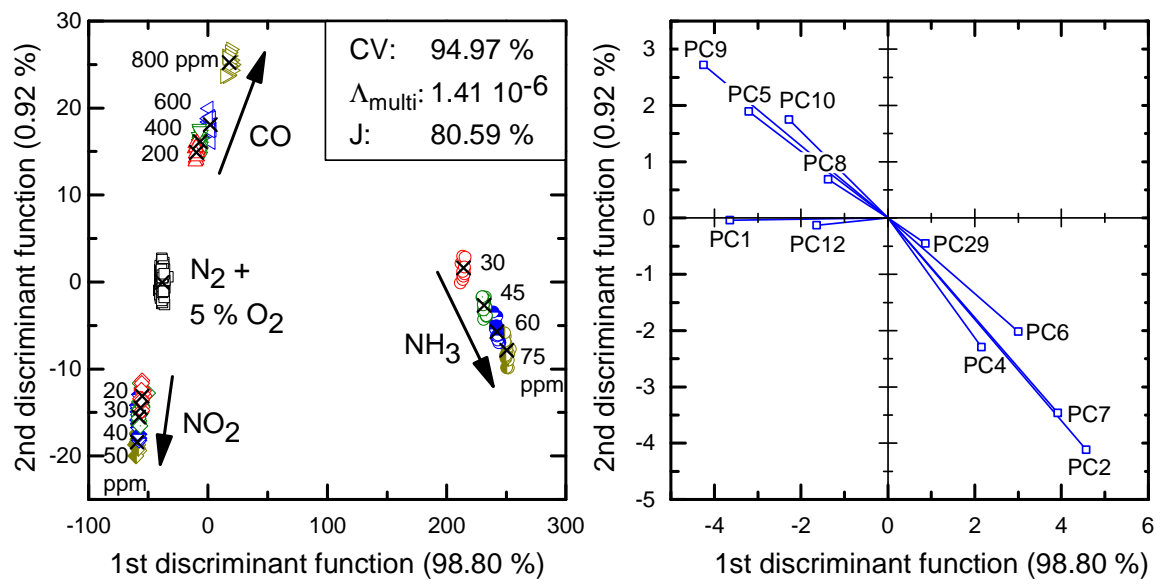

Fig. $8.40 \quad$ (a) LDA scatter plot showing the discrimination of $\mathrm{CO}, \mathrm{NO}_{2}$, and $\mathrm{NH}_{3}$ with four concentrations each when the first 36 principal components are used as features. The PCA was calculated based on extracted features. (b) Corresponding loadings plot. Only features with an average coefficient greater than 0.8 are shown.

\subsubsection{Stability and Influence of Normalization}

Besides the discrimination itself, an important issue is always the stability of the model over time. In Section 8.3.1, it was mentioned that every cycle has been standardized, in order to reduce the influence of sensor drift and scaling effects. Stability of the discrimination is thus closely related to the applied pre-processing.

In Fig. 8.41 LDA scatter plots showing the discrimination of $\mathrm{CO}, \mathrm{NO}_{2}$, and $\mathrm{NH}_{3}$ are given (open symbols) when features from all parts of the combined cycle are used and different kinds of pre-processing were applied. Each group combines four concentrations of the corresponding gas, i.e., 200, 400, 600, and $800 \mathrm{ppm} \mathrm{CO}, 20$, 30, 40, and $50 \mathrm{ppm} \mathrm{NO}_{2}$, and 30, 45, 60, and $75 \mathrm{ppm} \mathrm{NH}_{3}$. The obtained LDA coefficients are then used to project unknown data of a measurement, i.e., evaluation data, performed five days after the first measurement. Evaluation data are marked by semi-solid symbols and the groups again combine the same four concentrations for each gas as before. In a next step, the projected data are classified by Mahalanobis distance classifier to determine the correct classification rate. Fig. 8.41a shows the discrimination when the raw data are used for feature extraction. The overall classification rate is almost $65 \%$. For carbon monoxide and ammonia, the 
corresponding groups strongly overlap which means that there is almost no drift. However, nitrogen dioxide and the carrier gas group overlap which leads to high misclassification.
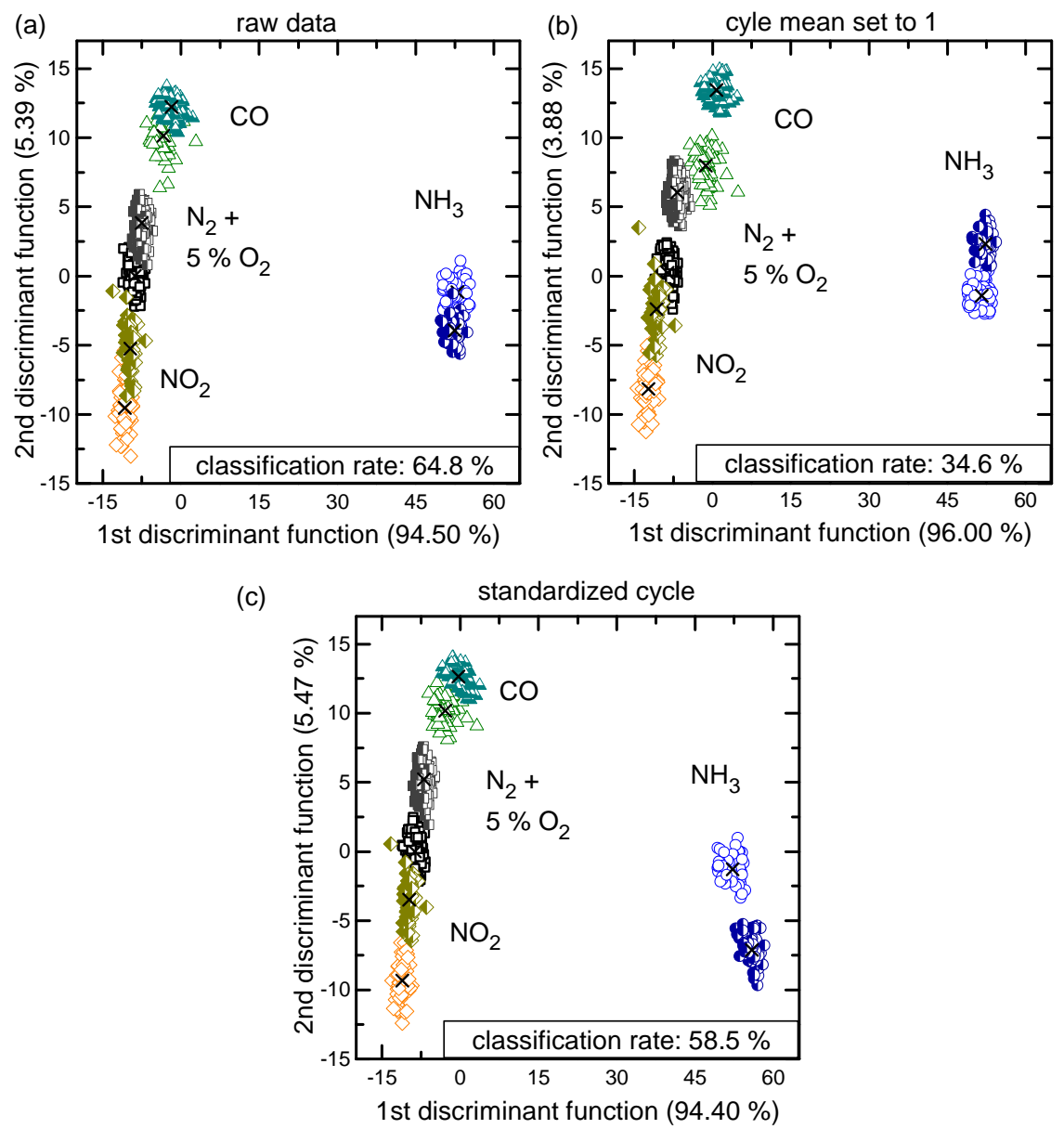

Fig. 8.41 LDA scatter plots showing the discrimination of $\mathrm{CO}, \mathrm{NO}_{2}$, and $\mathrm{NH}_{3}$. Each group consists of four concentrations. Training data are marked by open symbols and test data of a second measurement performed five days later by semi-solid symbols. The overall correct classification rate is obtained by a Mahalanobis distance classifier. (a) Raw data, (b) normalization by setting cycle mean to 1 , and (c) standardized cycle, i.e., setting cycle mean to 0 and standard deviation to 1 .

Typically, the applied normalization to TCO data is setting the cycle mean to 1 . The resulting LDA scatter plot is shown in Fig. 8.41 b. As can be observed there is a shift 
between the test and evaluation groups for all gases. Normally, that would be defined as sensor drift but since it is much less visible in the raw data, the shift in the normalized data means that the normalization itself amplifies noise and / or drift. The classification rate of $35 \%$ is also much lower compared to the raw data, $65 \%$. Another option to treat the data is standardization; the result is shown in Fig. 8.41c. The classification rate of, $58 \%$, is significantly better as for normalization but still not as good as for the raw data. Although, these results indicate that normalization or standardization is not needed and even more, impair the discrimination, normalization or standardization might be beneficial when not measuring under controlled lab conditions. In field measurements the baseline of the sensor can vary quite a lot which then requires certain pre-processing steps. Normalization can, for instance, suppress the influence of interference to some extent, e.g., humidity, if it changed the sensor response differently to the test gas. However, this needs to be tested and validated in each case. As a conclusion for the combined temperature and gate bias cycle, it can be stated that, in the studied example, standardization of the cycle is an appropriate way for pre-processing while the often used normalization of setting the cycle mean to 1 is insufficient. It was mentioned earlier that normalization also means a loss of information which is proven by the results presented in this section.

\subsubsection{Drift Compensation by Extended Training}

In the previous section, it is shown that the discrimination of the different gases independent of the concentration is affected by sensor drift. The drift, in particular, for carbon monoxide and ammonia is rather small, but still visible when applying the built model to new data a few days later. Thus, reduction of the influence of drift which leads to misclassification by normalization fails. Nevertheless, another possibility is to intentionally include the sensor drift in the model, i.e., the training. The idea behind this is that if the model knows how sensor drift affects the discrimination and the classification, it can be suppressed. This could simply be achieved by combining different training sets, i.e., enlarging the training data. As discussed in Section 7.4.2, as much information as possible should be included in the training. This could, for instance, be done by using several concentrations of each 
gas in order to allow discrimination independently of the concentration as it was shown in the previous section. Another example is to combine different levels of humidity in order to be nearly independent of this. Different concentrations of each gas and different level of humidity can be included in a single test measurement and then included in the training phase in the signal processing. However, to adopt this strategy for compensating sensor drift, it is important to combine at least two different measurements (data sets) which have been recorded at different points in time. Alternatively, the sensor can be aged artificially, e.g., by extra heat treatment.

However, in order to prove the concept two identical data sets where the second one has been recorded five days after the first one are combined, i.e., the number of observations or cycles is extended. Fig. 8.42 shows again the discrimination of $\mathrm{CO}$, $\mathrm{NO}_{2}$, and $\mathrm{NH}_{3}$ independent of the concentration when the data are standardized similar to Fig. 8.41c. For the training, the combined data set is used and the data are marked by open symbols.

For the evaluation, a third measurement which was performed five days after the second one is used, cf. Fig. 8.42.

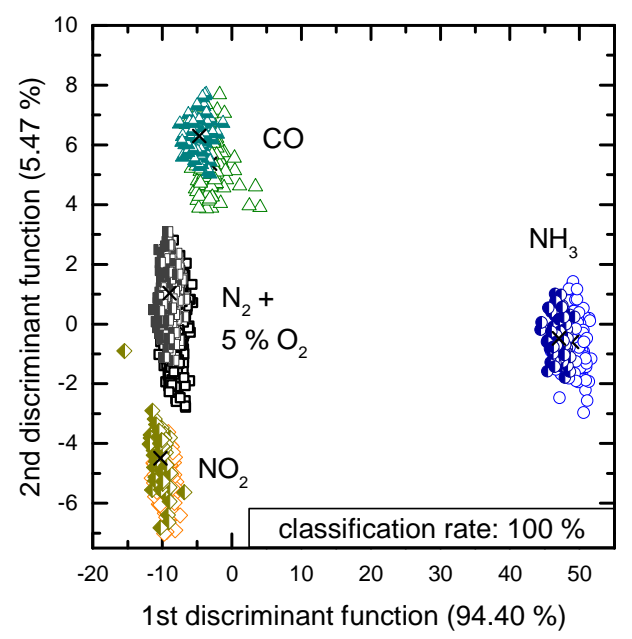

Fig. 8.42 LDA scatter plot showing the discrimination of $\mathrm{CO}, \mathrm{NO}_{2}$, and $\mathrm{NH}_{3}$ independent of concentration when the data are standardized first. Training data are combined of two measurements and marked by open symbols. Test data of a third measurement performed five days after the training are marked with semi-solid symbols and are classified by a Mahalanobis distance classifier. 
As evidently shown in the scatter plot, cf. Fig. 8.42, when using extended training data, i.e., intentionally including sensor drift in the training, the projected data groups are almost ideally overlapping with their corresponding training groups. The correct classification rate for this projection using a Mahalanobis distance classifier is $100 \%$. Hence, sensor drift can effectively be compensated by using extended training [291]. It should be noted that perfect classification can be achieved also when the raw data or normalized data are used. Therefore, using extended training outperforms pre-processing. Nevertheless, in real applications probably a combination of adequate pre-processing and extended training is needed to increase stability.

\subsubsection{Conclusion Extended Virtual Multi-sensor Approach}

In this sub-chapter, a novel method to operate SiC-FETs, i.e., combination of temperature and gate bias cycled operation, has been suggested. It was presented that by combining temperature and gate bias cycled operation the selectivity of SiC-FET gas sensors can be further enhanced. Additionally, sensor drift can effectively be compensated by extended training.

Quantification of, e.g., carbon monoxide is possible by using features from either the temperature cycle, i.e., the outer cycle, or one of the gate bias cycles, i.e., the inner cycle. However, the separation of the different groups is best when using features from all parts of the combined cycle. Discrimination of $\mathrm{CO}, \mathrm{NO}_{2}$, and $\mathrm{NH}_{3}$ independent of the concentration is possible. By using feature selection the dimension of the problem can be reduced significantly. Additionally, it was shown by feature selection that features from different parts of the combined cycle are important for the discrimination.

Discriminant Analysis of Principal Components is a suitable method for dynamically operated SiC-FETs. Similar results regarding the quality of the discrimination could be achieved. However, for well-conditioned data, the extra step of transforming the standard features in the PCA space is not necessary.

The applied pre-processing has a pronounced impact on the discrimination and its stability. This was shown by validating the built LDA model using a data set 
obtained a few days after. By this, it was suggested that normalization in fact impairs the stability, and leads to a loss of discriminatory information. Standardization outperforms normalization and leads to fairly good results regarding stability.

Sensor drift can be compensated by extended training independent of the applied preprocessing. Combining at least two different data sets, which were obtained at different points in time, and thus, include the drift, is a very promising technique to enhance the stability of the discrimination, i.e., to compensate sensor drift. The suggested method can be applied to any kind of chemical gas sensor.

Consequently, the proposed methodology of combining TCO and GBCO is a real extension of the virtual multi-sensor approach as indicated by generic lab measurements. 



\section{Conclusion and Outlook}

In this chapter, the main findings of this PhD project are summarized, cf. Section 9.1, and contribution to the scientific community is highlighted, cf. Section 9.2. At the end, an outlook for future work is given, cf. Section 9.3.

\subsection{Conclusion}

In this joint $\mathrm{PhD}$ project, different areas of chemical sensing are combined and with that, a strong collaboration between the Lab for Measurement Technology and the Division of Applied Sensor Science has been established. In the beginning of the thesis, the basics and state of the art of field effect transistors as well as signal processing are reviewed. The methodology of dynamic operation, i.e., temperature cycling, has systematically been developed for field effect based gas sensors. Temperature cycled operation, TCO, of field effect transistors has been applied to two different applications. Additionally, the virtual multi-sensor approach has been extended by gate bias cycling. The selectivity could be improved further by a combination of temperature and gate bias cycling.

In a first step, requirements for a new hardware platform have been defined to allow dynamic operation, i.e., temperature and bias cycling, as well as simultaneous readout of the sensor response. Based on the given requirements, a new hardware board was developed by $3 S \mathrm{GmbH}$ - Sensors, Signal Processing, Systems. Field effect transistors have a large number of variable parameters. A dedicated LabVIEW program has thus been developed which allows a precise control and read-out of the SiC-FET sensors together with the 3S-hardware, which is the basis for all further studies.

At the Lab for Measurement Technology, LMT, mainly Linear Discriminant Analysis has so far been used for evaluation of multi-dimensional data. However, at 
the beginning of this $\mathrm{PhD}$ project, the existing MATLAB tools for pre-processing and evaluation were highly limited and tailored to former applications with resistive type metal oxide gas sensors. Therefore, user-friendly, versatile graphical user interfaces, GUIs, for feature extraction and for evaluation with several different algorithms have been developed. Much effort has been spent on a GUI for Linear Discriminant Analysis, LDA, and Principal Component Analysis, PCA, which offers easy evaluation including cross-validation and classification of new data, and some possibilities for feature selection. Besides LDA and PCA, new algorithms, e.g., regression algorithms and Support Vector Machines, and software tools for those have been introduced expanding the LMT-toolbox considerably ${ }^{38}$.

In preliminary studies, the suitability of temperature cycling on field effect transistors has been proven by both qualitative analyses for discrimination of individual gases as well as quantitative determination of gas concentrations of typical exhaust gases. The results have been validated and the stability is in a reasonable range for chemical sensors.

For gas sensitive field effect transistors, not only temperature influences the sensor response but also the applied bias. However, gate bias modulation on SiC-FETs was not reported in literature. From temperature cycled operation of resistive type metal oxide, MOX, sensors, it is known that the occurrence of hysteresis is an indication that discriminatory information is contained in the transient response. In order to study the influence of a changing gate bias on the sensor properties, slow gate bias ramps have been used. Results have proven that the additional information is contained in the transient when changing the bias. The hysteresis depends on the slope of the bias ramp, the starting and end points, and the operating temperature. The shape of the hysteresis curves is strongly gas dependent and discrimination of carbon monoxide and ammonia are easily possible. The sensing mechanism behind is not completely understood yet but results in different ambient mixtures, i.e., changes in oxygen and / or humidity, suggest that at least two different effects are taking place when changing the bias. Flipping of trap states and spill-over effects on the sensor surface are conceivable, and maybe the adsorption and desorption rates are also altered. However, deeper analyses are necessary to come to a final conclusion.

\footnotetext{
${ }^{38}$ Software tools for regression analysis [276] and Support Vector Machines [275] have been developed as part of a master and a bachelor thesis which have been supervised by me during my $\mathrm{PhD}$ studies.
} 
The results of these preliminary studies are very promising and the basis for more complex and ambitious applications, like $\mathrm{NO}_{\mathrm{x}}$ detection in a varying background of other exhaust gases. An optimized temperature cycle has been developed based on temperatures of maximum sensitivity of $\mathrm{NO}$ and $\mathrm{NO}_{2}$ toward the background mixture. For developing an evaluation strategy, a standard background consisting of $600 \mathrm{ppm}$ carbon monoxide, $50 \mathrm{ppm}$ ethene, and $60 \mathrm{ppm}$ ammonia in an atmosphere of $10 \%$ oxygen in nitrogen was defined. Quantification of $\mathrm{NO}_{\mathrm{x}}$ in a constant background is possible by using LDA and Partial Least Squares Regression, PLSR. However, the quality of the discrimination is slightly better for LDA. A hierarchical approach can effectively be used to suppress the influence of a changing background. In a first step, the background gas which has changed most is defined. For the discrimination it is important that the training set contains as much information as possible. Thus, each background group consists of three $\mathrm{NO}_{\mathrm{x}}$ concentrations in a high and a low concentration of the corresponding background gas. This makes the discrimination more robust. Based on this decision, the $\mathrm{NO}_{\mathrm{x}}$ concentration in the particular background is determined. The suggested hierarchical strategy to suppress the influence of a changing background is versatile and can be transferred to other applications, e.g. sulfur dioxide monitoring [97].

As a second application, silicon carbide field effect transistors have been tested for indoor air quality applications, IAQ, i.e., for on-demand ventilation control. The challenge in this application is the selective detection of volatile organic compounds, VOC, in the low parts-per-billion, ppb, range. A simple temperature cycle with three temperature plateaus has been chosen. Formaldehyde, naphthalene, and benzene which have been selected as typical indoor air pollutants, can be detected with sufficiently high sensor responses in the relevant concentration range. For ondemand ventilation control, it is important to determine the main pollutant exceeding its threshold. A LDA model which separates the three VOCs from a "below threshold" groups has successfully been built. Besides LDA, Partial Least Squares Discriminant Analysis, PLS-DA, has been studied as well since this algorithm directly performs one-vs-all classification with an easily adjustable classification threshold. Although, the quality of the discrimination is lower than for LDA with a Mahalanobis distance classifier, the possibility to tweak the classifier makes PLS-DA attractive for this application. Quantification of the target VOCs above their 
threshold and independent of the level of relative humidity is possible. Naphthalene can be discriminated and quantified best while benzene on the other hand is very challenging. Quantification of naphthalene in a high background of ethanol, i.e., $1 \mathrm{ppm}$, is possible but also calls for a hierarchical strategy since ethanol has a strong impact on the sensor response. In conclusion, SiC-FETs are suitable candidates for IAQ applications, especially for the detection of naphthalene and to some extent also formaldehyde. Reliable detection of benzene is currently not possible. Optimization of the sensing layers and the used temperature cycle may solve this issue.

The virtual multi-sensor approach has been extended by gate bias cycling, particularly a combination of temperature and gate bias cycled operation. Features from all parts of a combined cycle are important for the discrimination of carbon monoxide, $\mathrm{CO}$, nitrogen dioxide, $\mathrm{NO}_{2}$, and ammonia, $\mathrm{NH}_{3}$, as shown by the loadings plot or by drawing the feature value over time. However, the selectivity is further improved by combining TCO and GBCO as proven by the discrimination of different $\mathrm{CO}$ concentrations. Additionally, the example of the discrimination of $\mathrm{CO}, \mathrm{NO}_{2}$, and $\mathrm{NH}_{3}$, has been used to enhance the signal processing. Feature selection can effectively be done by comparing the value of the average coefficients. The average coefficient is the numerical representation of the loadings plot but it does not provide any information about redundant features in contrast to the loadings plot. Thus, feature selection preferably should be done first on the average coefficient to determine the most significant features and then, the loadings plot is used to eliminate redundant features in order to reduce the dimension further. Moreover, the loadings plot can be used for studying the influence of each feature on the discrimination. As a measure of the quality of the discrimination, the cross-validation rate is used. Statistical parameters like the multivariate Wilks' Lambda are difficult to interpret and can only be used to compare scatter plots relative to each other. The cluster separability $J$ and the discrimination criterion indicate wherever the problem is ill-conditioned or not, and allow a relative comparison of different LDA scatter plots.

In case the number of features is high compared to the number of observations or, if it can be suspected that features are correlated, feature transformation prior to the discrimination is an effective method to overcome this. Transformation is done by 
calculating a PCA first, followed by Discriminant Analysis of Principal Components. However, for well-conditioned problems this extra step is usually not necessary.

Normalization does not necessarily compensate for sensor drift. The kind of normalization or standardization needs to be tested for each sensor, chosen cycle, and application. In case of the combined temperature and gate bias cycle studied in this project, standardization outperforms multiplicative normalization. A different but very effective approach to compensate for sensor drift is by extended training. The idea behind is to intentionally include the sensor drift in the training, i.e., combine data sets recorded at two different points in time. In general, preparing the training set is the most crucial and important step in order to build a robust and stable pattern recognition model.

In conclusion, this dissertation enlarges the LMT-toolbox considerably by a new sensor technology and several new methods for data evaluation: First, a new sensor technology was successfully studied and its great potential has been demonstrated by two challenging case studies. Second, new algorithms, e.g., Partial Least Squares Regression and Partial Least Squares-Discriminant Analysis, have been implemented and their suitability has been proven by lab measurements. Third, versatile methods for feature selection and feature transformation have been introduced which can be used for all types of chemical sensors. Fourth, drift compensation by extended training is a powerful approach to build a robust pattern recognition model, and in general, a comprehensive training set is the basis for a robust discrimination. And fifth, the virtual multi-sensor approach has been enlarged by bias modulation which boosts the selectivity of the sensors further.

Also the portfolio of the Div. of Applied Sensor Science has been expanded by the above mentioned methods, i.e., the virtual multi-sensor approach, including temperature and bias cycling and appropriate signal processing based on multivariate statistics. 


\subsection{My Contribution to the Scientific Community}

In the present thesis, the methodology of the virtual multi-sensor is transferred and extended to the area of field effect based gas sensing. It is the first time that temperature cycling is systematically studied for gas sensitive field effect transistors. Both quantitative and qualitative discrimination of typical exhaust gases is possible as proven by lab measurements. Additionally, gate bias cycling is introduced which is a novel way to operated SiC-FETs and, thus, an extension of the virtual multisensor approach. Gas significant hysteresis is found when sweeping the applied gate bias providing additional, discriminatory information from the transient behavior. The occurring effects are not completely understood yet, but results have proven that the selectivity of the sensors is increased considerably. The suggested methodology is versatile and can be transferred to any kind of field effect based sensor.

A hierarchical approach is used for quantification of nitrogen oxides, $\mathrm{NO}_{\mathrm{x}}$, in a varying background of other typical exhaust gases. In a first step, the change in the background mixture is determined. For this first decision, it is important that as much information as possible is contained in the training phase in order to make the discrimination robust. Thus, each group contains data from different $\mathrm{NO}_{\mathrm{x}}$ concentrations in a mixture with low and high concentrations of one of the background gases. After determination of the background, the concentration of the target gas is determined by using a polynomial fit of the group centroids of a few training groups, i.e., concentrations, in the LDA scatter plot. The suggested hierarchical strategy has been used in field measurements for the quantification of sulfur dioxide in a desulfurization unit [97]. Therefore, the suggested methodology to compensate for a varying background is not only suitable under lab conditions but also in the field.

Silicon carbide based field effect transistors have so far mainly been used in harsh environments, e.g., as ammonia sensors for control of SCR systems and operated directly in the exhaust stream. Additionally, these sensors are now applied to a new application, i.e., indoor air quality, in which ultra-low concentrations, i.e., in the low 
parts-per-billion range, of volatile organic compounds need to be detected. For on demand ventilation control, the main air pollutant exceeding its guideline value is determined first. For that, a "below threshold" group has been defined which contains data from pure air and concentrations of each target gas below its guideline value in order to make the determination robust. Discrimination is done by Linear Discriminant Analysis as well as by Partial Least Squares - Discriminant Analysis which is a new algorithm in the field of virtual multi-sensors.

The selectivity of the sensors can be increased further by combining temperature and gate bias cycled operation. Several feature selection methods have proven that features from all parts of the combined cycle are significant for the discrimination. This can be seen as a validation of the suggested approach which poses a real extension of the existing virtual multi-sensor approach.

Besides the sensors and their operating modes, new methods in terms of signal processing are suggested. Feature selection strategies which are partly known from electronic noses are successfully transferred to virtual multi-sensors. The most suitable method is feature selection based on the loadings plot and the average coefficient. For a large number of features, Discriminant Analysis of Principal Components, which performs Principal Component Analysis first and uses the resulting, orthogonal principal components as input for LDA, is a powerful method also for virtual multi-sensors. It was found that the quality of the discrimination is best expressed by the correct cross-validation rate rather than by statistical values like the multivariate Wilks' Lambda or the cluster separability.

Different kinds of normalization are studied and for the combination of temperature and gate bias cycled operation, standardization outperforms multiplicative normalization. However, which kind of normalization leads to the best results depends on various parameters and needs to be studied in each case. Regarding drift compensation, a new method is suggested based on extended training. Sensor drift is intentionally included in the training phases, i.e., in building the discrimination model. Preliminary results suggest that this method is outperforms normalization and can be applied to any kind of gas sensor. 


\subsection{Outlook}

Although, this thesis provides a comprehensive study on dynamically operated field effect transistors as well as on new methods for the signal processing, there are several open questions left which are worth to be studied in the future.

The combination of temperature and gate bias cycled operation has a great potential and increases the selectivity of the sensor considerably. However, the origin of the selectivity increase is not entirely understood yet. Future studies should therefore address the influence of a changing gate bias on the sensor properties. This, for instance, can be done by mass spectroscopy measurements in which the reaction products on the sensor surface are studied. If the gate bias influences the chemical reactions taking place on the surface, or if the adsorption and desorption rates are altered, it will be detectable in the mass spectra. Another option is surface analysis done by, e.g., surface potential measurements, scanning electron microscopy, SEM, Diffuse Reflectance Infrared Fourier Transform Spectroscopy, DRIFT, or transmission electron microscopy, TEM. The change in bias can influence the number of adsorption sites on the sensing layer, and/ or lead to spill-over effects which can be proven by surface analysis studies.

Not only fundamental studies regarding the gate bias are worth to study, but also applying the new method of TCO and GBCO to real applications. Although, TCO leads to fairly good results with respect to the discrimination and quantification of volatile organic compounds, there is still room for improvement. Thus, an optimization of the virtual multi-sensor approach for the selective detection of VOC should be investigated. Besides the optimization of the combined cycle itself, also the applied normalization and extracted features need to be optimized. As mentioned in the thesis, the normalization has a strong impact on the result of the discrimination. Generally, normalization or standardization is crucial and needs to be investigated more intensively and systematically in the future.

Several methods for manual feature extraction and selection have been presented in this thesis. The next step is the automatization of these methods, i.e., automatization 
which supports the user in finding the optimal feature set. One possibility is to look at the correlation between feature value and gas exposure.

It was shown that the influence of drift can effectively be reduced by merging two data sets, i.e., intentionally including drift in the training phase. However, repeating comprehensive gas profiles takes a lot of time and is often not easily possible. Hence, considerations regarding design of experiment, i.e., what has to be included in the measurement and what can be left out, should be made. Probably, it is sufficient to repeat just certain parts of a gas profile in order to include sensor drift in the training phase by merging two data sets. Design of experiment is not only important for drift compensation by extended training but generally in the training phase, especially for changing background mixtures.

Quantification of VOCs by Partial Least Squares Regression, and Linear Discriminant Analysis indicates a non-linear behavior of the sensor system. Thus, besides these linear algorithms, also non-linear algorithms like Support Vector Machines, SVM, and Support Vector Regression, SVR, should be considered. The necessary software tools have been developed during this $\mathrm{PhD}$ project but have not been applied to a specific application, like VOC detection, yet.

Due to given hardware limitations, the sensor has been operated in the linear region of the transistor which, according to the basics of a gas sensitive FET, offer a lower response as compared to the saturation region. In future studies, the saturation region should be used and temperatures of maximum sensor response should be determined. Especially, if transistors with novel sensing layers and / or insulators are used, the optimal operating temperatures need to be found first.

Another interesting topic is poisoning of SiC-FET gas sensors. For resistive type metal oxides gas sensors it is well known that silicone and sulfur containing substances block reactive sites. There are indications that sensors run in TCO actually get poisoned quicker than constantly heated sensors. Most often the poisoning is irreversible and makes a replacement of the sensor necessary. For SiC-FETs, particularly dynamically operated SiC-FETs, there is only limited knowledge on that.

Finally, the dynamically operated SiC-FETs should be applied in field measurements, e.g., for indoor air quality applications. Measuring under well- 
controlled lab conditions is always the first step. Field measurements are important to demonstrate the suitability of the sensor and the suggested virtual multi-sensor approach for real applications. As an example, the methodology of a hierarchical strategy to compensate for a changing background and a fit of the groups centroids in a LDA model for determining the concentration of a target gas, as presented in this thesis for $\mathrm{NO}_{\mathrm{x}}$, has been used for the development of a $\mathrm{SO}_{2}$ sensor [97]. A SiC-FET was installed in a desulfurization pilot unit in a power plant in Växjö, Sweden. Besides the $\mathrm{SO}_{2}$ concentration, also the background mixture changes continuously, mainly the oxygen and humidity level as well as the hydrogen chloride concentration, which makes a direct determination of the $\mathrm{SO}_{2}$ concentration not possible. Thus, a two-layer hierarchical approach, similar to the $\mathrm{NO}_{\mathrm{x}}$ sensing presented in this thesis, was chosen. In a first step, four background scenarios are defined and discriminated by LDA. Based on this decision, a second LDA together with an exponential fit of the group centroids corresponding to different $\mathrm{SO}_{2}$ concentrations is performed in order to determine the $\mathrm{SO}_{2}$ concentration. Fig. 9.1 shows the output of the model (second LDA and exponential fit) together with the reference instrument.

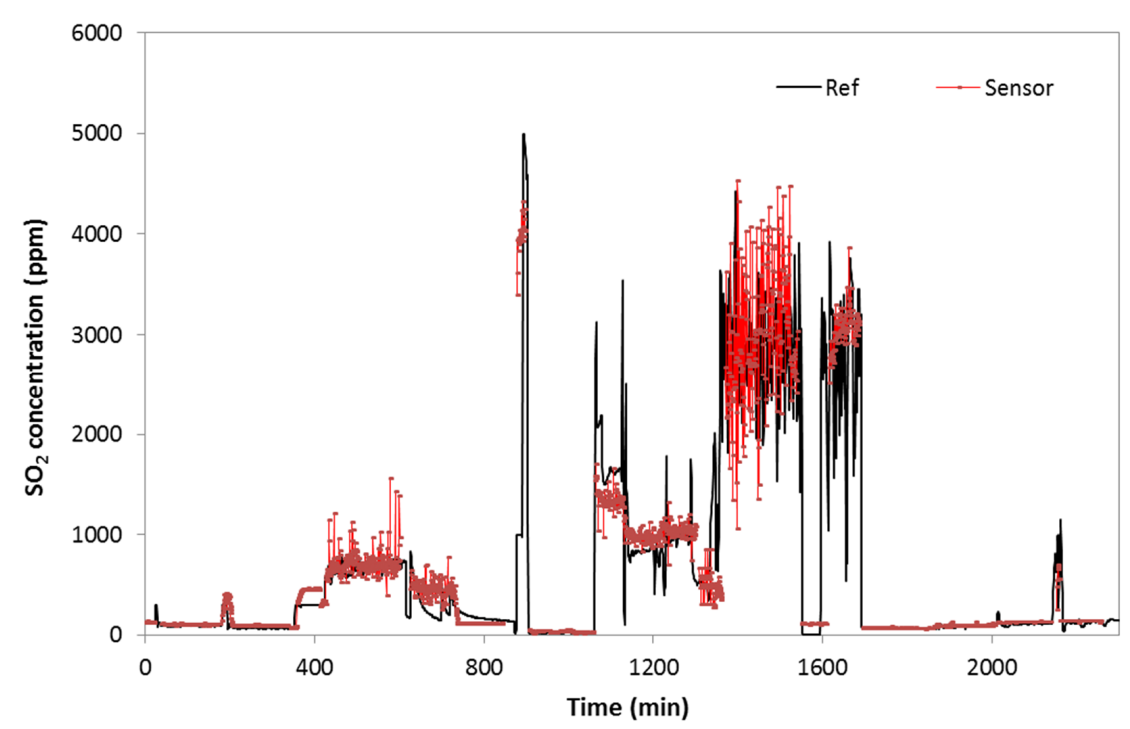

Fig. 9.1 Sulfur dioxide concentration in a pilot plant measurement. The signal from the reference instrument (black solid line) is compared to the sensor signal processed by using exponential fit from a LDA model (red dotted line). Reproduced from [97] with kind permission from Elsevier. 
As can be observed, the SiC-FET sensor follows the reference instrument very well which proves the suitability of a hierarchical approach in the field.

In addition to field measurements, algorithms for online and real time control of, e.g., desulphurization units or ventilation systems, need to be developed. 



\section{References}

[1] AppliedSensor. Freshen your home, save energy, "Indoor Air Quality Modules Freshen Homes, Save Energy (application note)," 2011.

Available: https://www.appliedsensor.com/2011/06/freshen-your-home-saveenergy/ Accessed: (2014, Dec. 17)

[2] T. Tille, "Requirements for gas sensors in automotive air quality applications," in Solid State Gas Sensors - Industrial Application, M. Fleischer and M. Lehmann, Eds. Berlin, Heidelberg: Springer Verlag, 2012, pp. 13-34.

ISBN: 978-3-642-28092-4

[3] M. Blaschke, T. Tille, P. Robertson, S. Mair, U. Weimar, and H. Ulmer, "MEMS gas-sensor array for monitoring the perceived car-cabin air quality," IEEE Sensors J., vol. 6, no. 5, pp. 1298-1308, 2006.

DOI: $10.1109 /$ JSEN.2006.881399

[4] SGX Sensortech. (2014, Dec. 17). Automotive air quality sensors. [Online]. Available: http://www.sgxsensortech.com/automotive-air-quality-sensors/

[5] AppliedSensor. (2014, Dec. 17). ACM air classification module. [Online]. Available:

https://www.appliedsensor.com/products/air_classification_module_acm/

[6] O. Ahmed, "Future building gas sensing applications," in Solid State Gas Sensors - Industrial Applications, M. Fleischer and M. Lehmann, Eds. Berlin, Heidelberg: Springer-Verlag, 2012, pp. 3-12.

ISBN: 978-3-642-28092-4

[7] Zafer Ankara, "Untersuchung zum Einsatz von mikrostrukturierten Halbleitergassensoren in der heutigen Gebäudebrandmeldetechnik," dissertation, Saarland University, Saarbrücken, Germany, 2008, Shaker 
Verlag.

ISBN: 978-3-8322-7310-1

[8] Peter Reimann, "Gasmesssysteme basierend auf Halbleitergassensoren für sicherheitskritische Anwendungen mit dem Ansatz der Sensorselbstüberwachung," dissertation, Saarland University, Saarbrücken, Germany, 2011, Shaker Verlag.

ISBN: 978-3-8440-0232-4

[9] R. Pohle, E. Simon, R. Schneider, M. Fleischer, R. Sollacher, H. Gao, K. Müller, P. Jauch, M. Loepfe, H.-P. Frerichs, and C. Wilbertz, "Fire detection with low power FET gas sensors," Sens. Actuators B, vol. 120, no. 2, pp. 669672, 2007.

DOI: $10.1016 /$ j.snb.2006.03.040

[10] D. Kohl, J. Kelleter, and H. Petig, "Detection of fires by gas sensors," in Sensors update, H. Baltes, J. Hesse, and J.G. Korvink, Eds. Weinheim: WileyVHY, 2001, ch. 2.2, pp. 161-224.

ISBN: 3-527-30360-X

[11] Joseph Wang, "Electrochemical sensing of explosives," Electroanalysis, vol. 19, no. 4, pp. 415-423, 2007.

DOI: $10.1002 /$ elan.200603748

[12] Vamsee K. Pamula, "Detection of explosives," in Handbook of Machine Olfaction, T.C. Pearce et al., Eds. Weinheim: Wiley-VCH, 2003, ch. 23, pp. 547-560.

ISBN: 3-527-30358-8

[13] A. Gramm and A. Schütze, "Sealing testing method for products incorporating a fluid (Verfahren und Vorrichtung zur Dichtheitsprüfung)," patent DE 10316332 B4, Dec. 7, 2006.

[14] A. Gramm, A. Schütze, and R. Gottfried, "Fast leak test based on gas sensors for direct integration in the manufacturing process," in SENSOR 2003, Nuremberg, Germany, May 13-15, 2003.

[15] C. Kübel, "Automotive hydrogen sensors: current and future requirements," in 
Solid State Gas Sensors - Industrial Applications, M. Fleischer and M. Lehmann, Eds. Berlin, Heidelberg: Springer-Verlag, 2012, pp. 35-38.

ISBN: 978-3-642-28092-4

[16] H.T. Nagle, R. Gutierrez-Osuna, B.G. Kermani, and S.S. Schiffman, "Environmental monitoring," in Handbook of Machine Olfaction, T.C. Pearce et al., Eds. Weinheim: Wiley-VCH, 2003, ch. 17, pp. 419-444.

ISBN: 3-527-30358-8

[17] H. Nanto and J.R. Stetter, "Introduction to chemosensors," in Handbook of Machine Olfaction, T.C. Pearce et al., Eds. Weinheim: Wiley-VCH, 2003, ch. 4, pp. 79-104.

ISBN: 3-527-30358-8

[18] J.W. Gardner and P.N. Bartlett, Electronic Noses: Principles and Applications. New York: Oxford University Press, 1999.

ISBN: 978-0198559559

[19] A. Schütze, A. Gramm, and T. Rühl, "Identification of organic solvents by a virtual multisensor system with hierarchical classification," IEEE Sensors J., vol. 4, no. 6, pp. 857-863, 2004.

DOI: 10.1109/JSEN.2004.833514

[20] The Regional Office for Europe of the World Health Organization. Health aspects of air pollution and review of EU policies: the REVIHAAP and HRAPIE projects, "Health aspects of air pollution in Europe," 2013.

Available: $\quad$ http://www.euro.who.int/en/health-topics/environment-andhealth/air-quality/publications/2013/review-of-evidence-on-health-aspects-ofair-pollution-revihaap-project-final-technical-report Accessed: (2014, Dec. 16)

[21] The Regional Office for Europe of the World Health Organization. Health aspects of air pollution and review of EU policies: the REVIHAAP and HRAPIE projects, "Review of evidence on health aspects of air pollution REVIHAAP project," 2013.

Available: $\quad$ http://www.euro.who.int/en/health-topics/environment-andhealth/air-quality/activities/health-aspects-of-air-pollution-and-review-of-eu- 
policies-the-revihaap-and-hrapie-projects Accessed: (2014, Dec. 16)

[22] WHO - World Health Organization, Air quality guidelines for Europe - global update 2005. Copenhagen, Danmark: WHO - The Regional Office for Europe, 2006.

ISBN: 9289021926

[23] WHO - World Health Organization, Air quality guidelines for Europe, 2nd ed. Copenhagen, Denmark: Organization, The Regional Office for Europe of the World Health, 2000.

ISBN: 9289013583

[24] WHO - The World Health Organization. Ambient (outdoor) air quality and health, "Fact sheet $\mathrm{N}^{\circ} 313, " 2014$.

DOI: http://www.who.int/mediacentre/factsheets/fs313/en/\#

Accessed: (2014, Dec. 16)

[25] WHO - The World Health Organization, Health effects of transport-related air pollution. Copenhagen, Danmark: WHO Regional Office for Europe, 2005.

ISBN: 92-890-1375-3

[26] S.C. Sofuoglu, G. Aslan, F. Inal, and A. Sofuoglu, "An assessment of indoor air concentrations and health risks of volatile organic compounds in three primary schools," Int. J. Hyg. Envir. Heal., vol. 214, pp. 36-46, 2011.

DOI: 10.1016/j.ijheh.2010.08.008

[27] M.R. Ashmore and C. Dimitroulopoulou, "Personal exposure of children to air pollution," Atmos. Environ., vol. 43, pp. 128-141, 2009.

DOI: 10.1016/j.atmosenv.2008.09.024

[28] European Union. EUR-LEX, "Regulation (EC) No 715/2007 of the European Parliament and of the Council," Official Journal of the European Union, no. 32007R0715, 2007.

Available:

http://eur-lex.europa.eu/legalcontent/DE/TXT/?uri=CELEX:32007R0715 Accessed: (2014, Dec. 09)

[29] Edward Jobson, "Future challenges in automotive emission control," Top. Catal., vol. 28, no. 1-4, pp. 191-199, 2004. 
DOI: 10.1023/B:TOCA.0000024350.93474.d1

[30] G.A. Bishop and D.H. Stedman, "Emissions of nitrogen dioxide from modern diesel vehicles," WIT Trans. on Ecology and Environment, vol. 116, pp. 247254, 2008.

DOI: 10.2495/AIR080261

[31] D.C. Carslaw, S.D. Beevers, E. Westmoreland, M.L. Williams, J.E. Tate, T. Murrells, J. Stedman, Y. Li, S. Grice, A. Kent, and I. Tsagatakis. Department of Environment, Food \& Rural Affairs, "Trends in NOx and NO2 emissions and ambient measurements in the UK," 2011.

Available: http://uk-air.defra.gov.uk/ Accessed: (2014, Dec. 16)

[32] European Environment Agency. "Air quality in Europe - 2012 report ," 2012. Available: http://www.eea.europa.eu/publications/air-quality-in-europe-2012 Accessed: (2014, Dec. 16)

[33] R. Burch, J.P. Breen, and F.C. Meunier, "A review of the selective reduction of NOx with hydrocarbons under lean-burn conditions with non-zeolitic oxide and platinum group metal catalysts," Appl. Catal. B, vol. 39, pp. 283-303, 2002.

DOI: $10.1016 / \mathrm{S} 0926-3373(02) 00118-2$

[34] M. Koebel, M. Elsener, and M. Kleemann, "Urea-SCR: A promising technique to reduce NOx emissions from automotive diesel engines," Catal. Today, vol. 59, pp. 335-345, 2000.

DOI: 10.1016/S0920-5861(00)00299-6

[35] D.A. Sarigiannis, S.P. Karakitsios, A. Gotti, I.L. Liakos, and A. Katsoyiannis, "Exposure to major volatile organic compounds and carbonyls in European indoor environments and associated health risk," Environ. Int., vol. 37, no. 4, pp. 743-765, 2011.

DOI: 10.1016/j.envint.2011.01.005

[36] H. Salonen, A.-L. Pasanen, S. Lappalainen, H. Riuttala, T. Tuomi, P. Pasanen, B. Bäck, and K. Reijula, "Volatile organic compounds and formaldehyde as explaining factors for sensory irritation in office environments," J. Occup. 
Environ. Hyg., vol. 6, pp. 239-247, 2009.

DOI: $10.1080 / 15459620902735892$

[37] Wilhelm Kirch, Encyclopedia of public health. Berlin, Germany: SpringerVerlag, 2008, p. 1303.

ISBN: 978-1-4020-5613-0

[38] S.J. Emmerich and A.K. Persily, State-of-the-art review of CO2 demand controlled ventilation technology and application.: NIST - National Institute of Standards and Technology, Mar. 2001, NISTIR 6729.

[Online]. http://fire.nist.gov/bfrlpubs/build01/art117.html

[39] J. Hodgkinson and R.P. Tatam, "Optical gas sensing: a review," Meas. Sci. Technol., vol. 24, p. 012004, 2013.

DOI: 10.1088/0957-0233/24/1/012004

[40] J. Zosel, W. Oelßner, M. Decker, G. Gerlach, and U. Guth, "The measurement of dissolved and gaseous carbon dioxide concentration," Meas. Sci. Technol., vol. 22, p. 072001, 2011.

DOI: $10.1088 / 0957-0233 / 22 / 7 / 072001$

[41] Thomas Schmid, "Photoacoustic spectroscopy for process analysis," Anal. Bioanal. Chem., vol. 384, pp. 1071-1086, 2006.

DOI: 10.1007/s00216-005-3281-6

[42] WHO - World Health Organization, WHO guidelines for indoor air quality: selected pollutants, The Regional Office for Europe of the World Health Organization, Ed. Copenhagen, Denmark, 2010.

ISBN: 9789289002134

[43] D.H. Do, H. van Langenhove, C. Walgraeve, S.F. Hyleeyesus, P. de Wispelaere, J. Dewulf, and K. Demeestere, "Volatile organic compounds in an urban environment: A comparison among Belgium, Vietnam, and Ethiopia," Int. J. Environ. Anal. Chem., vol. 93, no. 3, pp. 298-314, 2013.

DOI: $10.1080 / 03067319.2011 .62070$

[44] L. Pérez-Lombard, J. Ortiz, and C. Pout, "A review on buildings energy consumption information," Energy Build., vol. 40, pp. 394-398, 2008. 
DOI: 10.1016/j.enbuild.2007.03.007

[45] SENSIndoor. (2014, Dec. 08). [Online].

Available: http://www.sensindoor.eu/

[46] ANSES-French Agency for Food, Environmental and Occupational Health \& Safety. Indoor Air Quality Guidelines (IAQGs),.

Available: https://www.anses.fr/en/content/indoor-air-quality-guidelines-iaqgs Accessed: (Dec. 2014)

[47] C. Bur, M. Andersson, A. Lloyd Spetz, and A. Schütze, "Detecting volatile organic compounds in the ppb range with gas sensitive platinum gate $\mathrm{SiC}$ field effect transistors," IEEE Sensors J., vol. 14, no. 9, pp. 3221-3228, 2014.

DOI: 10.1109/JSEN.2014.2326693

[48] La ministre de l'écologie, du développement durable, des transports et du logement (France). Legifrance, "Décret n 2011-1727 du 2 décembre 2011 relatif aux valeurs-guides pour l'air intérieur pour le formaldéhyde et le benzène," Journal Officiel De La République Francaise, no. 2011-1727, p. 20529, 2011.

Available:

http://www.legifrance.gouv.fr/affichTexte.do?cidTexte=JORFTEXT00002490 9119\&dateTexte\&categorieLien $=$ id

[49] German Federal Ministry for the Environment, Nature Conservation and Nuclear Safety. Ad-hoc Arbeitsgruppe für Innenraumrichtwerte, "Krebserzeugende Wirkung von Formaldehyd - Änderung des Richtwertes für die Innenraumluft von 0,1 ppm nicht erforderlich," Bundesgesundheitsblatt, vol. 49, p. 1169, 2006.

DOI: $10.1007 / \mathrm{s} 00103-006-0081-\mathrm{x}$

Available: $\quad$ www.umweltbundesamt.de/themen/gesundheit/kommissionenarbeitsgruppen/ad-hoc-arbeitsgruppe-innenraumrichtwerte Accessed: (2014, Dec. 04)

[50] German Federal Ministry for the Environment, Nature Conservation and Nuclear Safety. Ad-Hoc Work. Group Indoor Air Guide Values, "Richtwerte für Naphthalin und Naphthalin-ähnliche Verbindungen in der Innenraumluft," 
Bundesgesundheitsblatt, vol. 56, pp. 1448-1459, 2013.

DOI: 10.1007/s00103-013-1836-9

Available:

http://www.umweltbundesamt.de/themen/gesundheit/kommissionen-

arbeitsgruppen/ad-hoc-arbeitsgruppe-innenraumrichtwerte Accessed: (2014, Dec. 04)

[51] German Federal Ministry for the Environment, Nature Conservation and Nuclear Safety. Ad-hoc-Arbeitsgruppe Innenraumrichtwerte, "Richtwerte für die Innenraumluft: Kohlenmonoxid," Bundesgesundheitsblatt, vol. 11, pp. 425428, 1997.

Available:

http://www.umweltbundesamt.de/themen/gesundheit/kommissionenarbeitsgruppen/ad-hoc-arbeitsgruppe-innenraumrichtwerte Accessed: (2014, Dec. 04)

[52] WHO - World Health Organization, WHO Air quality guidelines for particulate matter, ozone, nitrogen dioxide, and sulfur dioxide. Geneva, Switzerland, 2006, WHO/SDE/PHE/OEH/06.02.

[53] WHO - World Health Organization, WHO handbook on indoor radon. Geneva, Switzerland, 2009.

ISBN: 9789241547673

[54] German Federal Ministry for the Environment, Nature Conservation and Nuclear Safety. Ad-hoc-Arbeitsgruppe Innenraumrichtwerte, "Richtwerte für die Innenraumluft: Toluol," Bundesgesundheitsblatt, vol. 39, pp. 416-421, 1996.

Available:

http://www.umweltbundesamt.de/themen/gesundheit/kommissionenarbeitsgruppen/ad-hoc-arbeitsgruppe-innenraumrichtwerte Accessed: (2014, Dec. 04)

[55] K. Koistinen, D. Kotzias, S. Kephalopoulos, C. Schlitt, P. Carrer, M. Jantunen, S. Kirchner, J. McLaughlin, L. Molhave, E.O. Fernandes, and B. Seifert, "The INDEX project: executive summary of an European Union project on indoor 
air pollutants," Allergy, vol. 63, pp. 810-819, 2008.

DOI: $10.1111 /$ j.1398-9995.2008.01740.x

[56] D. Puglisi, J. Eriksson, C. Bur, A. Schütze, A. Lloyd Spetz, and M. Andersson, "Catalytic metal-gate field effect transistors based on $\mathrm{SiC}$ for indoor air quality control," J. Sens. Sens. Syst., vol. 4, pp. 1-8, 2015.

DOI: $10.5194 /$ jsss-4-1-2015

[57] T. Salthammer, S. Mentese, and R. Marutzky, "Formaldehyde in the indoor environment," Chem. Rev., vol. 110, pp. 2536-2572, 2010.

DOI: $10.1021 / \mathrm{cr} 800399 \mathrm{~g}$

[58] U.S. Government Accountability Office. "Formaldehyde in textiles: While levels in clothing generally appear to be low, allergic contact dermatitis is a health issue for some people," Report to Congressional Committees, no. GAO10-875, 2010.

Available: http://www.gao.gov/products/GAO-10-875 Accessed: (2014, Dec. 04)

[59] JRC - Joint Research Centre of the European Comission, European survey on the release of formaldehyde from textiles. Luxemburg: Office for Official Publications of the European Communities, 2007.

ISBN: 978-92-79-05215-6

[Online]. http://publications.jrc.ec.europa.eu/repository/handle/JRC36150

[60] OEHHA - Office of Environmental Health Harzard Assessment. Air Toxicology and Epidemiology, "Appendix D.1 individual acute, 8-hour, and chronic reference exposure level summaries," Technical support document for the derivation of noncancer reference exposure levels and RELs for six chemicals, 2008, updated 2013 and 2014.

Available: http://oehha.ca.gov/air/hot_spots/rels_dec2008.html Accessed: (2014, Dec. 04)

[61] Clifford P. Weisel, "Benzene exposure: An overview of monitoring methods and their findings," Chem.-Biol. Interact., vol. 184, no. 1-2, pp. 58-66, 2010. DOI: 10.1016/j.cbi.2009.12.030 
[62] R. Preuss, J. Angerer, and H. Drexler, "Naphthalene - an environmental and occupational toxicant," Int. Arch. Occup. Environ. Health, vol. 76, pp. 556576, 2003.

DOI: $10.1007 / \mathrm{s} 00420-003-0458-1$

[63] National Pesticide Information Center. "Naphthalene - technical fact sheet". Available: http://npic.orst.edu/factsheets/naphtech.html Accessed: (2014, Dec. 04)

[64] German Federal Ministry for the Environment, Nature Conservation and Nuclear Safety. Ad-hoc-Arbeitsgruppe Innenraumrichtwerte, "Richtwerte für die Innenraumluft: erste Fortschreibung des Basisschemas," Bundesgesundheitsblatt, vol. 55, pp. 279-290, 2012.

DOI: $10.1007 / \mathrm{s} 00103-011-1420-0$

Available:

http://www.umweltbundesamt.de/themen/gesundheit/kommissionenarbeitsgruppen/ad-hoc-arbeitsgruppe-innenraumrichtwerte Accessed: (2014, Dec. 04)

[65] S.M. Sze, Physics of Semiconductor Devices, 2nd ed. New York, Chichester, Brisbane, Toronto, Singapore: John Wiley \& Sons, 1981.

ISBN: 978-0471098379

[66] D.A. Neamen, Semiconductor Physics and Devices, 4th ed. New York: McGraw-Hill International Edition, 2012.

ISBN: 978-007-108902-9

[67] I. Lundström, A. Van den Berg, B.H. Van der Schoot, H.H. Van der Vlekkert, M. Armgarth, and C.J. Nylander, "Field Effect Chemical Sensors," in Sensors A Comprehensive Survey, W. Göpel, J. Hesse, and J.N. Zemel, Eds. Weinheim: VCH, 1991, ch. 10, pp. 467-528.

ISBN: 3-527-26768-9

[68] M. Andersson, A. Lloyd Spetz, and R. Pearce, "Recent trends in silicon carbide ( $\mathrm{SiC}$ ) and graphene-based gas sensors," in Semiconductor gas sensors, R. Jaaniso and O.K. Tan, Eds. Cambridge: Woodhead Publishing, 2013, ch. 4, pp. 117-158. 
ISBN: 978-0-85709-236-6

[69] U. Tietze and Ch. Schenk, Halbleiterschaltungstechnik, 13th ed. Berlin, Heidelberg: Springer Verlag, 2010.

ISBN: 978-3642016219

[70] P. Horowitz and W. Hill, The Art of Electronics, 2nd ed. Cambridge, New York, Melbourne, Madrid, Cape Town, Singapore, Sao Paulo: Campridge University Press, 1989.

ISBN: 0-521-37095-7

[71] A. Lloyd Spetz, M. Skoglundh, and L. Ojamäe, "FET gas-sensing mechanism, experimental and theoretical studies," in Solid State Gas Sensing, E. Comini, G. Faglia, and G. Sberveglieri, Eds. New York: Springer, 2009, ch. 4, pp. 153179.

ISBN: 978-0-387-09664-3

[72] P. Bergveld, "Development of an ion-sensitive solid-state device for neurophysiological measurements," IEEE Trans. Biomed. Eng., vol. 17, no. 1, pp. 70-71, 1970.

DOI: $10.1109 /$ TBME.1970.4502688

[73] I. Lundström, S. Shivaraman, C. Svensson, and L. Lundkvist, "A hydrogensensitive MOS field-effect transistor," Appl. Phys. Lett., vol. 26, no. 2, pp. 5557, 1975.

DOI: $10.1063 / 1.88053$

[74] I. Lundström, S. Shivaraman, and C. Svensson, "A hydrogen-sensitive Pd-gate MOS transistor," J. Appl. Phys., vol. 46, no. 9, pp. 3876-3881, 1975.

DOI: $10.1063 / 1.322185$

[75] P. Bergveld, "Thirty years of ISFETOLOGY: What happened in the past 30 years and what may happen in the next 30 years," Sens. Actuators B, vol. 88, no. 1, pp. 1-20, 2003.

DOI: $10.1016 / \mathrm{S} 0925-4005(02) 00301-5$

[76] I. Lundström, H. Sundgren, F. Winquist, M. Eriksson, C. Krantz-Rülcker, and A. Lloyd Spetz, "Twenty-five years of field effect gas sensors research in 
Linköping," Sens. Actuators B, vol. 121, pp. 247-262, 2007.

DOI: $10.1016 /$ j.snb.2006.09.046

[77] M. Eriksson and L.-G. Ekedahl, "Hydrogen adsorption states at the PdSiO2 interface and simulation of the response of the response of a Pd metal-oxidesemiconductor hydrogen sensor," J. Appl. Phys., vol. 83, no. 8, pp. 3947-3951, 1998.

DOI: $10.1063 / 1.367150$

[78] M. Wallin, H. Grönbeck, A. Lloyd Spetz, M. Eriksson, and M. Skoglundh, "Vibrational analysis of H2 and D2 adsorption on Pt/SiO2," J. Phys. Chem. B, vol. 109, pp. 9581-9588, 2005.

DOI: $10.1021 / j p 044759 z$

[79] M. Löfdahl, C. Utaiwasin, A. Carlsson, I. Lundström, and M. Eriksson, "Gas response dependence on gate metal morphology of field-effect devices," Sens. Actuators B, vol. 80, no. 3, pp. 183-192, 2001.

DOI: $10.1016 / \mathrm{S} 0925-4005(01) 00886-3$

[80] A. Spetz, M. Armgarth, and I. Lundström, "Hydrogen and ammonia response of metal-silicon dioxide-silicon structures with thin platinum gates," J. Appl. Phys., vol. 64, no. 3, pp. 1274-1283, 1989.

DOI: $10.1063 / 1.341846$

[81] C. Wilbertz, H.-P. Frerichs, I. Freund, and M. Lehmann, "Suspended-Gateand Lundstrom-FET integrated on a CMOS-chip," Sens. Actuators A, vol. 123124, pp. 2-6, 2005.

DOI: $10.1016 /$ j.sna.2005.03.042

[82] A. Lloyd Spetz and S. Savage, "Advances in SiC field effect gas sensors," in Silicon Carbide - Recent major advances, W.J. Choyke, H. Matsunami, and G. Pensl, Eds. Berlin: Springer Verlag, 2003, pp. 869-896.

ISBN: 3-540-40458-9

[83] A. Spetz, A. Arbab, and I. Lundström, "Gas sensors for high temperature operation based on metal oxide silicon carbide (MOSiC) devices," in Proc. Eurosensors VI, San Sebastian, Spain, 1992. 
[84] A. Arbab, A. Spetz, and I. Lundström, "Gas sensors for high temperature operation based on metal oxide silicon carbide (MOSiC) devices," Sens. Actuators B, vol. 15, no. 1-3, pp. 19-23, 1993.

DOI: $10.1016 / 0925-4005(93) 85022-3$

[85] G.W. Hunter, P.G. Nuedeck, G.D. Jefferson, C.G. Madzsar, C.C. Liu, and H.Q. Wu, "The development of hydrogen sensor technology," NASA Techn. Memor., vol. 106 141, 1993.

[86] S.M. Savage, A. Konstantinov, A.M. Saroukan, and C. Harris, "High temperature 4H-SiC FET for gas sensing applications," Mater. Sci. Forum, vol. 338-342, pp. 1431-1434, 2000.

DOI: 10.4028/www.scientific.net/MSF.338-342.1431

[87] F. Ren and S.J. Pearton, "Recent advances in wide bandgap semiconductorbased gas sensors," in Semiconductor Gas Sensors, R. Jaaniso and O.K. Tan, Eds. Cambridge: Woodhead Publishing, 2013, ch. 5, pp. 159-219.

ISBN: 978-0-85709-236-6

[88] A. Lloyd Spetz, A. Baranzahi, P. Tobias, and I. Lundström, "High temperature sensors based on metal-insulator-silicon carbide devices," Phys. Status Solidi A, vol. 162, no. 1, pp. 496-511, 1997.

DOI: $10.1002 / 1521-396 X(199707) 162: 13.0 . C O ; 2-C$

[89] H. Wingbrant, I. Lundström, and A. Lloyd Spetz, "The speed of response of MISiCFET devices," Sens. Actuators B, vol. 93, pp. 286-294, 2003.

DOI: $10.1016 / \mathrm{S} 0925-4005(03) 00228-4$

[90] A. Lloyd Spetz and M. Andersson, "Technology and application opportunities for SiC-FET gas sensors," in Solid State Gas Sensors - Industrial Applications, M. Fleischer and M. Lehmann, Eds. Berlin, Heidelberg: Springer Verlag, 2012, pp. 189-214.

ISBN: 978-3-642-28092-4

[91] H. Wingbrant, H. Svenningstorp, P. Salomonsson, P. Tengström, I. Lundström, and A. Lloyd Spetz, "Using a MISiCFET device as a cold start sensor," Sens. Actuators B, vol. 93, no. 1-3, pp. 295-303, 2003. 
DOI: 10.1016/S0925-4005(03)00227-2

[92] O. Larsson, A. Göras, J. Nytomt, C. Carlsson, A. Lloyd Spetz, T. Artursson, M. Holmberg, I. Lundström, L.G. Ekedahl, and P. Tobias, "Estimation of air fuel ratio of individual cylinders in SI engines by means of MISiC sensor signals in a linear regression model," in SAE 2002, Detroit, USA, 4-7 March 2002, 2002-01-0847, also selected for SAE 2002 Transactions - Journal of Engines.

[93] M. Andersson, P. Ljung, M. Mattson, M. Löfdahl, and A. Lloyd Spetz, "Investigations on the possibilities of a MISiCFET sensor system for OBD and combustion control utilizing different catalytic gate materials," Top. Catal., vol. 30/31, pp. 365-368, 2004.

DOI: 10.1023/B:TOCA.0000029776.18603.74

[94] L. Unéus, T. Artursson, M. Mattsson, P. Ljung, R. Wigren, P. Martensson, M. Holmberg, I. Lundström, and A. Lloyd Spetz, "Evaluation of on-line flue gas measurements by MISiCFET and metal-oxide sensors in boilers," IEEE Sensors J., vol. 5, no. 1, pp. 75-81, 2005.

DOI: $10.1109 /$ JSEN.2004.839132

[95] M. Andersson, L. Everbrand, A. Lloyd Spetz, T. Nyström, M. Nilsson, C. Gauffin, and H. Svensson, "A MISiCFET based gas sensor system for combustion control in small-scale wood fired boilers," in Proc. IEEE Sensors 2007, Atlanta, USA, Oct. 28-31, 2007, pp. 962-965.

DOI: 10.1109/ICSENS.2007.4388563

[96] Z. Darmastuti, C. Bur, P. Möller, R. Rahlin, N. Lindqvist, M. Andersson, A. Schütze, and A. Lloyd Spetz, "SiC - FET based SO2 sensor for power plant emission applications," Sens. Actuators B, vol. 194, pp. 511-520, 2014.

DOI: 10.1016/j.snb.2013.11.089

[97] Z. Darmastuti, C. Bur, N. Lindqvist, M. Andersson, A. Schütze, and A. Lloyd Spetz, "Hierarchical methods to improve the performance of the SiC-FET as SO2 sensors in flue gas desulphurization systems," Sens. Actuators B, vol. 206, pp. 609-616, 2015. 
DOI: $10.1016 /$ j.snb.2014.09.113

[98] H. Wingbrant, H. Svenningstorp, P. Salomonsson, D. Kubinski, J.H. Visser, M. Löfdahl, and A. Lloyd Spetz, "Using a MISiC-FET sensor for detecting NH3 in SCR systems," IEEE Sensors J., vol. 5, no. 5, pp. 1099-1105, 2005.

DOI: 10.1109/JSEN.2005.854489

[99] C. Bur, M. Bastuck, A. Schütze, J. Juuti, A. Lloyd Spetz, and M. Andersson, "Characterization of ash particles with a microheater and gas-sensitive $\mathrm{SiC}$ field-effect transistors," J. Sens. Sens. Syst., vol. 3, pp. 305-313, 2014.

DOI: www.j-sens-sens-syst.net/3/305/2014/

[100] A. Lloyd Spetz, J. Huotari, C. Bur, B. Bjorklund, J. Lappalainen, H. Jantunen, A. Schütze, and M. Andersson, "Chemical sensor systems for emission control from combustions," Sens. Actuators B, vol. 187, pp. 184-190, 2013.

DOI: 10.1016/j.snb.2012.10.078

[101] Jiri Janata, "Chemically sensitive field effect transistor," US Patent 4,411,741, October 25, 1983.

[102] I. Eisele, B. Flietner, and K.-T. Doll, "Verfahren zum spacerfreien, hybriden Aufbau von Luftspalt und Gate von Suspended Gate Feldeffekttransistoren (SGFET) sowie nach dem Verfahren hergestellte Bauelemente," German Patent DE 4239 319.1, October 2, 1996.

[103] H. Lorenz, M. Peschke, H. Riess, J. Janata, and I. Eisele, "New suspended gate FET technology for physical deposition of chemically sensitive layers," Sens. Actuators B, vol. 23, no. 1-3, pp. 1023-1026, 1990.

DOI: 10.1016/0924-4247(90)87082-T

[104] I. Eisele and T. Knittel, "Work function based field effect devices for gas sensing," in Encyclopedia of Sensors, C.A. Grimes, E.C. Dickey, and M.V. Pishko, Eds. Los Angeles: American Scientific Publishers, 2006, ch. 10, pp. 473-490.

ISBN: 1-58883-066-7

[105] C. Senft, P. Iskra, and I. Eisele, "Theory and application of suspended gate FET gas sensors," in Solid State Gas Sensors - Industrial Applications, M. 
Fleischer and M. Lehmann, Eds. Heidelberg: Springer, 2012, pp. 77-112.

ISBN: 978-3-642-28092-4

[106] M. Burgmair, H.-P. Frerichs, M. Zimmer, M. Lehmann, and I. Eisele, "Field effect transducers for work function gas measurements: device improvements and comparison of performance," Sens. Actuators B, vol. 95, no. 1-3, pp. 183$188,2003$.

DOI: $10.1016 / \mathrm{S} 0925-4005(03) 00412-\mathrm{X}$

[107] Z. Gergintschew, P. Kornetzky, and D. Schipanski, "The capacitively controlled field effect transistor (CCFET) as a new low power gas sensor," Sens. Actuators B, vol. 36, no. 1-3, pp. 285-289, 1996.

DOI: $10.1016 / \mathrm{S} 0925-4005(97) 80083-4$

[108] C. Baratto, E. Comini, G. Faglia, and G. Sberveglieri, "The power of nanomaterial approaches in gas sensors," in Solid State Gas Sensors Industrial Applications, M. Fleischer and M. Lehmann, Eds. Heidelberg: Springer, 2012, pp. 53-78.

ISBN: 978-3-642-28092-4

[109] Eduard Llobet, "Gas sensors using carbon nanomaterials: A review," Sens. Actuators B, vol. 179, pp. 32-45, 2013.

DOI: $10.1016 /$ j.snb.2012.11.014

[110] E. Comini, C. Baratto, I. Concina, G. Faglia, M. Falasconi, M. Ferroni, V. Galstyan, E. Gobbi, A. Ponzoni, A. Vomiero, D. Zappa, V. Sberveglieri, and G. Sberveglieri, "Metal oxide nanoscience and nanotechnology for chemical sensors," Sens. Actuators B, vol. 179, pp. 3-20, 2013.

DOI: $10.1016 /$ j.snb.2012.10.027

[111] J. Kong, N.R. Franklin, C. Zhou, M.G. Chapline, S. Peng, K. Cho, and H. Dai, "Nanotube molecular wires as chemical sensors," Science, vol. 287, pp. 622$625,2000$.

DOI: $10.1126 /$ science.287.5453.622

[112] M. Bögner, A. Fuchs, K. Scharnagl, R. Winter, T. Doll, and I. Eisele, "Electrical field impact on the gas adsorptivity of thin metal oxide films," 
Appl. Phys. Lett., vol. 73, p. 2524, 1998.

DOI: $10.1063 / 1.122503$

[113] Z. Fan and J.G. Lu, "Gate-refreshable nanowire chemical sensors," App. Phys. Lett., vol. 86, p. 123510, 2005.

DOI: $10.1063 / 1.1883715$

[114] Noboru Yamazoe, "New approaches for improving semiconductor gas sensors," Sens. Actuators B, vol. 5, no. 1-4, pp. 7-19, 1991.

DOI: 10.1016/0925-4005(91)80213-4

[115] R. Pearce, R. Yakimova, J. Eriksson, L. Hultman, M. Andersson, and A. Lloyd Spetz, "Development of FETs and resistive devices based on epitaxially grown single layer graphene on $\mathrm{SiC}$ for highly sensitive gas detection," Mat. Science Forum, vol. 717-720, pp. 687-690, 2012.

DOI: 10.4028/www.scientific.net/MSF.717-720.687

[116] Y. Paska and H. Haick, "Interactive effect of hysteresis and surface chemistry on gated silicon nanowire gas sensors," CS Appl. Mater. Interfaces, vol. 4, no. 5, pp. 2604-2617, 2012.

DOI: $10.1021 / \mathrm{am} 300288 \mathrm{z}$

[117] Hans Lüth, Solid Surfaces, Interfaces and Thin Films, 4th ed. Berlin, Heidelberg, New York: Springer, 2001.

ISBN: 3-540-42331-1

[118] K. Oura, V.G. Lifshits, A.A. Saranin, A.V. Zotov, and M. Katayama, Surface Science. Berlin, Heidelberg, New York: Springer-Verlag, 2003.

ISBN: 3-540-00545-5

[119] Peter W. Atkins, Physikalische Chemie, 3rd ed. Weinheim, New York, Chichester, Brisbane, Singapore, Toronto: Wiley-VHC, 2001.

ISBN: 3-527-30236-0

[120] I. Chorkendorff and J.W. Niemantsverdriet, Concepts of Modern Catalysis and Kinetics. Weinheim, Germany: WILEY-VCH Verlag GmbH \& Co. KGaA, 2003. 
ISBN: 3-527-30574-2

[121] S. Brunauer, P.H. Emmett, and E. Teller, "Adsorption of gases in multimolecular layers," J. Am. Chem. Soc., vol. 60, no. 2, pp. 309-319, 1938. DOI: $10.1021 / \mathrm{ja} 01269 \mathrm{a} 023$

[122] A. Oprea, N. Bârsan, and U. Weimar, "Work function changes in gas sensitive materials," Sens. Actuators B, vol. 142, pp. 470-493, 2009.

DOI: $10.1016 /$ j.snb.2009.06.043

[123] E. Comini, G. Faglia, and G. Sberveglieri, "Electrical-based gas sensing," in Solid State Gas Sensing, E. Comini, G. Faglia, and G. Sberveglieri, Eds. New York: Springer, 2009, ch. 2, pp. 47-107.

ISBN: 978-0-387-09664-3

[124] J. Fogelberg, M. Eriksson, H. Dannetun, and L.-G. Petersson, "Kinetic modeling of hydrogen adsorption/absorption in thin films on hydrogensensitive field-effect devices: Observation of large hydrogen-induced dipoles at the Pd-SiO2 interface," J. Appl. Phys., vol. 78, pp. 988-996, 1995.

DOI: $10.1063 / 1.360293$

[125] H.M. Dannetun, L.-G. Petersson, D. Söderberg, and I. Lundström, "A hydrogen sensitive Pd-MOS structure working over a wide pressure range," Appl. Surf. Sci. , vol. 17, pp. 259-264, 1984.

DOI: $10.1016 / 0378-5963(84) 90014-X$

[126] M. Eriksson, I. Lundström, and L.-G. Ekedahl, "A model of the Temkin isotherm behavior for hydrogen adsorption at $\mathrm{Pd}-\mathrm{SiO} 2$ interfaces," J. Appl. Phys., vol. 82, pp. 3143-3146, 1997.

DOI: $10.1063 / 1.366158$

[127] A. Salomonsson, M. Eriksson, and H. Dannetun, "Hydrogen interaction with platinum and palladium metal-insulator-semiconductor devices," J. Appl. Phys., vol. 98, p. 014505, 2005.

DOI: $10.1063 / 1.1953866$

[128] L.-G. Ekedahl, M. Eriksson, and I. Lundström, "Hydrogen sensing mechanisms of metal-insulator interfaces," Acc. Chem. Res., vol. 31, pp. 249- 
256, 1998.

DOI: $10.1021 / \operatorname{ar} 970068 s$

[129] J. Schalwig, G. Müller, U. Karrer, M. Eickhoff, O. Ambacher, M. Stutzmann, L. Görgens, and G. Dollinger, "Hydrogen response mechanism of Pt-GaN Schottky diodes ," Appl. Phys. Lett., vol. 80, pp. 1222-1224, 2002.

DOI: $10.1063 / 1.1450044$

[130] O. Weidemann, M. Hermann, G. Steinhoff, H. Wingbrant, A. Lloyd Spetz, M. Stutzmann, and M. Eickhoff, "Influence of surface oxides on hydrogensensitive Pd:GaN Schottky diodes," Appl. Phys. Lett., vol. 83, pp. 773-775, 2003

DOI: $10.1063 / 1.1593794$

[131] M. Eriksson, A. Salomonsson, I. Lundström, D. Briand, and A.E. Åbom, "The influence of insulator surface properties on the hydrogen response of fieldeffect gas sensors," J. Appl. Phys., vol. 98, p. 034903, 2005.

DOI: $10.1063 / 1.1994941$

[132] F. Winquist, A. Spetz, M. Armgarth, C. Nylander, and I. Lundström, "Modified palladium metal-oxide-semiconductor structures with increased ammonia gas sensitivity," Appl. Phys. Lett., vol. 43, pp. 839-841, 1983.

DOI: $10.1063 / 1.94514$

[133] A. Spetz, M. Armgarth, and I. Lundström, "Optimization of ammonia-sensitive metal-oxide-semiconductor structures with platinum gates," Sens. Actuators B, vol. 11, no. 4, pp. 349-365, 1987.

DOI: $10.1016 / 0250-6874(87) 80075-6$

[134] M. Eriksson and L.-G. Ekedahl, "The influence of CO on the response of hydrogen sensitive Pd-MOS devices," Sens. Actuators B, vol. 42, pp. 217-223, 1997.

DOI: $10.1016 / \mathrm{S} 0925-4005(97) 80338-3$

[135] M. Eriksson and L.-G. Petersson, "Spillover of hydrogen, oxygen and carbon monoxide in oxidation reactions on $\mathrm{SiO} 2$ supported Pd," Surf. Sci., vol. 311, no. 1-2, pp. 139-152, 1994. 
DOI: 10.1016/0039-6028(94)90485-5

[136] J. Schalwig, P. Kreisl, S. Ahlers, and G. Müller, "Response mechanism of SiCbased MOS field effect gas sensors," IEEE Sensors J., vol. 2, no. 5, pp. 394402, October 2002.

DOI: 10.1109/JSEN.2002.806214

[137] T. Yamagucchi, T. Kiwa, K. Tsukada, and K. Yokosawa, "Oxygen interference mechanism of platinum-FET hydrogen gas sensor," Sens. Actuators A, vol. 136, no. 1, pp. 244-248, 2007.

DOI: $10.1016 /$ j.sna.2006.11.026

[138] S. Nakagomi, P. Tobias, A. Baranzahi, I. Lundström, P. Mårtensson, and A. Llozd Spetz, "Influence of carbon monoxide, water and oxygen on high temperature catalytic metal-oxide-silicon carbide structures," Sens. Actuators $B$, vol. 45, pp. 183-191, 1997.

DOI: 10.1016/S0925-4005(97)00292-X

[139] E. Becker, M. Andersson, M. Eriksson, A. Lloyd Spetz, and M. Skoglundh, "Study of the sensing mechanism towards carbon monoxide of platinum-based field effect sensors," IEEE Sensors J., vol. 11, no. 7, pp. 1527-1534, 2011. DOI: 10.1109/JSEN.2010.2099652

[140] G. Müller, J. Schalwig, P. Kreisl, A. Helwig, E. Obermeier, O. Weidemann, M. Stutzmann, and M. Eickhoff, "High-temperature operated field-effect gas sensors," in Encyclopedia of Sensors, C.A. Grimes, E.C. Dickey, and V. Pishko, Eds. Los Angeles: American Scientific Publishers, 2006, ch. 4, pp. 431-458.

ISBN: $1-58883-062-4$

[141] M. Andersson, H. Wingbrant, and A. Lloyd Spetz, "Study of the CO response of SiC based field effect gas sensors," in Proc. IEEE Sensors 2005, Irvine, CA, USA, Oct. 30 - Nov. 3, 2005.

DOI: $10.1109 /$ ICSENS.2005.1597647

[142] R. Dagonnier and J. Nuyts, "Oscillating CO oxidation on a Pt surface," $J$. Chem. Phys., vol. 65, pp. 2061-2065, 1976. 
DOI: $10.1063 / 1.433389$

[143] R.E. Lagos, B.C. Sales, and H. Suhl, "Theory of oscillatory oxidation of carbon monoxide over platinum," Surf. Sci., vol. 82, no. 2, pp. 525-539, 1979. DOI: 10.1016/0039-6028(79)90209-7

[144] L. Li, W. Wlodarski, K. Galatsis, and D.A. Powell, "A study of CO sensors with oscillatory response," Sens. Actuators B, vol. 96, pp. 610-614, 2003.

DOI: 10.1016/j.snb.2003.07.003

[145] M.C. Wheeler, R.E. Cavicchi, and S. Semancik, "Tin oxide microsensor arrays as probes for oscillatory $\mathrm{CO}$ oxidation reaction on supported platinum," $J$. Phys.Chem., vol. 111, pp. 3328-3332, 2007.

DOI: $10.1021 / j p 067173 \mathrm{~g}$

[146] M.C. Wheeler, J.E. Tiffany, R.M. Walton, R.E. Cavicchi, and S. Semancik, "Chemical crosstalk between heated gas microsensor elements operating in close proximity," Sens. Actuators B, vol. 77, pp. 167-176, 2001.

DOI: $10.1016 / \mathrm{S} 0925-4005(01) 00689-\mathrm{X}$

[147] M. Andersson and A. Lloyd Spetz, "Tailoring of field effect gas sensors for sensing of non-hydrogen containing substances from mechanistic studies on model systems," in Proc. IEEE Sensors 2009, Christchurch, New Zealand, Oct. 25-28, 2009, pp. 2031-2035.

DOI: 10.1109/ICSENS.2009.5398282

[148] M. Ali, V. Cimalla, V. Lebedev, T. Stauden, G. Ecke, V. Tilak, P. Sandvik, and O. Ambacher, "SiC-based FET for detection of NOx and $\mathrm{O} 2$ using InSnOx as gate material," Sens. Actuators B, vol. 122, pp. 182-186, 2007.

DOI: $10.1016 /$ j.snb.2006.05.021

[149] S. Nakagomi and A. Lloyd Spetz, "Gas sensor device based on catalytic metalmetal oxide-SiC structure," in Encyclopedia of Sensors, C.A. Grimes, E.C. Dickey, and M.V. Pishko, Eds. Los Angeles: American Scientific Publishers, 2006, ch. 4 , pp. $155-170$.

ISBN: $1-58883-060-8$

[150] R. Gutierrez-Osuna, H.T. Nagle, B. Kermani, and S.S. Schiffman, "Signal 
conditioning and preprocessing," in Handbook of Machine Olfaction, T.C. Pearce et al., Eds. Weinheim: Wiley-VCH, 2003, ch. 5, pp. 105-132.

ISBN: 3-527-30358-8

[151] International Union of Pure and Applied Chemistry (IUPAC). Compendium of Chemical Terminology, , vol. 2.3.2, version: 2.3.2, 2012.

Available: http://goldbook.iupac.org/

[152] A. D'Amico and C. Di Natale, "A contribution on some basic definitions of sensors properties," IEEE Sensors J., vol. 1, no. 3, pp. 183-190, 2001.

DOI: 10.1109/JSEN.2001.954831

[153] Jan Mitrovics, "Auswerteverfahren für Gassensorarrays," dissertation, University of Tübingen, Tübingen, Germany, 2004.

[154] W. Göpel and K.-D. Schierbaum, "Definitions and typical examples," in Sensors - A Comprehensice Survey, W. Göpel, J. Hesse, and J.N. Zemel, Eds. Weinheim: VCH Verlagsgesellschaft, 1991, ch. 1, pp. 1-27.

ISBN: 3-527-26768-9

[155] Matthias Otto, Chemometrics, 2nd ed. Weinheim: Wiley-VCH, 2007. ISBN: 978-3-527-31418-8

[156] International Bureau of Weights and Measures. VIM3: International Vocabulary of Metrology, "JCGM 200:2012 - International vocabulary of metrology - basic and general concepts and associated terms (VIM)," 2012. Available: http://www.bipm.org/en/publications/guides/vim.html Accessed: (2015, Jan. 23)

[157] K. Persaud and G. Dodd, "Analysis of discrimination mechanisms in the mammalian olfactory system using a model nose," Nature, vol. 299, pp. 352$355,1982$.

DOI: $10.1038 / 299352 \mathrm{a} 0$

[158] T.C. Pearce et al., Eds., Handbook of Machine Olfaction. Weinheim: WileyVCH, 2003.

ISBN: 3-527-30358-8 
[159] Gardner, J.W.; Bartlett, P.N., "Sensors and sensory sytems for an electronic nose," NATO ASI Series: Applied Science, no. 12, 1992, ISBN: 9780792316930 .

[160] Ingemar Lundström, "Picture the smell," Nature, vol. 406, pp. 682-683, 2000. DOI: $10.1038 / 35021156$

[161] I. Lundström, T. Ederth, H. Kariis, H. Sundgren, A. Spetz, and F. Winquist, "Recent developments in field-effect gas sensors," Sens. Actuators B, vol. 23, pp. 127-133, 1995.

DOI: $10.1016 / 0925-4005(94) 01280-U$

[162] U. Tisch and H. Haick, "Nanomaterials for cross-reactive sensor arrays," MRS Bulletin, vol. 35, pp. 797-803, 2010.

DOI: $10.1557 / \mathrm{mrs} 2010.509$

[163] F. Röck, N. Barsan, and U. Weimar, "Electronic Nose: current status and future trends," Chem. Rev., pp. 705-725, 2008.

DOI: $10.1021 / \mathrm{cr} 068121 \mathrm{q}$

[164] H. Eicker, "Method and apparatus for determining the concentration of one gaseous component in a mixture of gases," US patent no. 4012692, 1977.

[165] A.P. Lee and B.J. Reedy, "Temperature modulation in semiconductor gas sensors," Sens. Actuators B, vol. 60, pp. 35-42, 1999.

DOI: $10.1016 / \mathrm{S} 0925-4005(99) 00241-5$

[166] W.M. Sears, K. Colbow, and F. Consadori, "Algorithms to improve the selectivity of thermally cycled tin oxide gas sensors," Sens. Actuators B, vol. 19, pp. 333-349, 1989.

DOI: 10.1016/0250-6874(89)87084-2

[167] W.M. Sears, K. Colbow, and F. Consadori, "General characteristics of thermally cycled tin oxide gas sensors," Semicond. Sci. Technol., vol. 4, pp. 351-359, 1989.

DOI: $10.1088 / 0268-1242 / 4 / 5 / 004$

[168] Eduard Llobet, "Temperature-modulated semiconductor gas sensors," in 
Encyclopedia of Sensors, C.A. Grimes, E.C. Dickey, and M.V. Pishko, Eds. Los Angeles: American Scientific Publishers, 2006, ch. 10, pp. 131-152.

ISBN: $1-58883-066-7$

[169] M. Baumbach, T. Kammerer, A. Sossong, and A. Schütze, "A new method for fast identification of gases and gas mixtures after sensor power up," in Proc. IEEE Sensors 2004, Vienna, Austria, Oct. 24-27, 2004, p. 1388.

DOI: 10.1109/ICSENS.2004.1426443

[170] A. Vergara, K.D. Benkstein, and S. Semancik, "Thermally-assisted transient analysis for reducing the response time of microhotplate gas sensors," in Proc. IEEE Sensors 2013, Baltimore, MD, USA, Nov. 3-6, 2013.

DOI: 10.1109/ICSENS.2013.6688156

[171] Thomas Kammerer, "Entwurf und Realisierung von Messplattformen zur effizienten Entwicklung intelligenter Gasmesssysteme," dissertation, Saarland University, Saarbrücken, Germany, 2005, Shaker Verlag.

ISBN: 978-3-8322-5000-3

[172] M. Schweizer-Berberich, M. Zdralek, U. Weimar, W. Göpel, T. Viard, D. Martinez, A. Seube, and A. Peyre-Lavigne, "Pulsed mode of operation and artificial neural network evaluation for improving the $\mathrm{CO}$ selectivity of $\mathrm{SnO} 2$ gas sensors," Sens. Actuators B, vol. 65, pp. 91-93, 2000.

DOI: $10.1016 / \mathrm{S} 0925-4005(99) 00333-0$

[173] X. Huang, J. Liu, D. Shao, Z. Pi, and Z. Yu, "Rectangular mode of operation for detecting pesticide residue," Sens. Actuators B, vol. 96, pp. 630-635, 2003. DOI: 10.1016/j.snb.2003.07.006

[174] Z. Ankara, T. Kammerer, and A. Schütze, "Low power virtual sensor array based on a micromachined gas sensor for fast discrimination between $\mathrm{H} 2, \mathrm{CO}$ and relative humidity," Sens. Actuators B, vol. 100, no. 1-3, pp. 240-245, 2004. DOI: $10.1016 /$ j.snb.2003.12.072

[175] S. Nakata, Y. Kaneda, H. Nakamura, and K. Yoshikawa, "Detection and quantification of $\mathrm{CO}$ gas based on the dynamic response of a ceramic sensor," Chem. Lett., vol. 20, no. 9, pp. 1505-1508, 1991. 
[176] S. Nakata and H. Nakamura, "New strategy for the development of a gas sensor based on the dynamic," Sens. Actuators B, vol. 8, pp. 187-189, 1992. DOI: $10.1016 / 0925-4005(92) 80179-2$

[177] S. Nakata, E. Ozaki, and N. Ojima, "Gas sensing based on the dynamic nonlinear responses of a semiconductor gas sensor: dependence on the range and frequency of a cyclic temperature change," Anal. Chim. Acta, vol. 361, pp. 93-100, 1998.

DOI: $10.1016 / \mathrm{S} 0003-2670(98) 00013-0$

[178] A. Vergara, E. Llobet, J. Brezmes, P. Ivanov, C. Cané, I. Gràcia, X. Vilanova, and X. Correig, "Quantitative gas mixture analysis using temperaturemodulated micro-hotplate gas sensors: Selection and validation of the optimal modulating frequencies," Sens. Actuators B, vol. 123, pp. 1002-1016, 2007.

DOI: $10.1016 /$ j.snb.2006.11.010

[179] A. Vergara, J.L. Ramirez, and E. Llobet, "Reducing power consumption via a discontinuous operation of temperature-modulated micro-hotplate gas sensors: Application to the logistics chain of fruit," Sens. Actuators B, vol. 129, pp. 311-318, 2008.

DOI: $10.1016 /$ j.snb.2007.08.029

[180] R. Gutierrez-Osuna, A. Gutierrez-Galvez, and N. Powar, "Transient response analysis for temperature-modulated chemiresistors," Sens. Actuators B, vol. 93, pp. 57-66, 2003.

DOI: 10.1016/S0925-4005(03)00248-X

[181] Y. Hiranaka, T. Abe, and H. Murata, "Gas-dependent response in the temperature transient of $\mathrm{SnO} 2$ gas sensors," Sens. Actuators B, vol. 9, pp. 177$182,1992$.

DOI: 10.1016/0925-4005(92)80213-H

[182] Y. Kato, K. Yoshikawa, and M. Kitora, "Temperature-dependent dynamic response enables the qualification and quantification of gases by a single sensor," Sens. Actuators B, vol. 40, no. 1, pp. 33-37, 1997.

DOI: 10.1016/S0925-4005(97)80196-7 
[183] S. Semancik and R. Cavicchi, "Kinetically controlled chemical sensing using micromachined structures," Acc. Chem. Res., vol. 31, pp. 279-287, 1998. DOI: $10.1021 / \operatorname{ar} 970071 b$

[184] T.A. Kunt, T.J. McAvoy, R.E. Cavicchi, and S. Semancik, "Optimization of temperature programmed sensing for gas identification using micro-hotplate sensors," Sens. Actuators B, vol. 95, pp. 24-43, 1998.

DOI: $10.1016 / \mathrm{S} 0925-4005(98) 00244-5$

[185] K.D. Benkstein, P.H. Rogers, C.B. Montgomery, C. Jin, B. Raman, and S. Semancik, "Analytical capabilities of chemiresistive microsensor arrays in a simulated Martian atmosphere," Sens. Actuators B, vol. 197, pp. 280-291, 2014.

DOI: $10.1016 /$ j.snb.2014.02.088

[186] S. Semancik, R.E. Cavicchi, M.C. Wheeler, J.E. Tiffany, G.E. Poirier, R.M. Walton, J.S. Suehle, B. Panchapakesan, and D.L. DeVoe, "Microhotplate platforms for chemical sensor research," Sens. Actuators B, vol. 77, no. 1-2, pp. 579-591, 2001.

DOI: $10.1016 / \mathrm{S} 0925-4005(01) 00695-5$

[187] T. Eklöv, P. Mårtensson, and I. Lundström, "Enhanced selectivity of MOSFET gas sensors by systematical analysis of transient parameters," Anal. Chim. Acta , vol. 353, pp. 291-300, 1997.

DOI: $10.1016 / \mathrm{S} 0003-2670(97) 87788-4$

[188] D. Briand, H. Wingbrant, H. Sundgren, B. Van der Schoot, L.-G. Ekedahl, I. Lundström, and N.F. de Rooij, "Modulated operating temperature for MOSFET gas sensors: hydrogen recovery time reduction and gas discrimination," Sens. Actuators B, vol. 93, pp. 276-285, 2003.

DOI: $10.1016 / \mathrm{S} 0925-4005(03) 00230-2$

[189] P. Kreisl, A. Helwig, G. Müller, E. Obermeier, and S. Soltier, "Detection of hydrocarbon species using silicon MOS field-effect transistors operated in a non-stationary temperature-pulse mode," Sens. Actuators B, vol. 106, no. 1, pp. 442-449, 2005. 
DOI: $10.1016 /$ j.snb.2004.09.004

[190] P. Kreisl, A. Helwig, A. Friedberger, G. Müller, E. Obermeier, and S. Soltier, "Detection of hydrocarbon species using silicon MOS capacitors operated in a non-stationary temperature pulse mode," Sens. Actuators B, vol. 106, no. 2, pp. 489-497, 2005.

DOI: $10.1016 /$ j.snb.2004.07.030

[191] E. Martinelli, D. Polese, A. Catini, A. D'Amico, and C. Di Natale, "Selfadapted temperature modulation in metal-oxide semiconductor gas sensors," Sens. Actuators B, vol. 161, pp. 534-541, 2012.

DOI: $10.1016 /$ j.snb.2011.10.072

[192] J. Fonollosa, L. Fernández, R. Huerta, A. Gutiérrez-Gálvez, and S. Marco, "Temperature optimization of metal oxide sensor arrays using Mutual Information," Sens. Actuators B, vol. 187, pp. 331-339, 2013.

DOI: $10.1016 /$ j.snb.2012.12.026

[193] R. Gutierrez-Osuna and A. Hierlemann, "Adaptive microsensor systems," Annu. Rev. Anal. Chem., vol. 3, pp. 255-276, 2010.

DOI: 10.1146/annurev.anchem.111808.073620

[194] A. Varpula, S. Novikov, A. Haarahiltunen, and P. Kuivalainen, "Transient characterization techniques for resistive metal-oxide gas sensors," Sens. Actuators B, vol. 159, pp. 12-26, 2011.

DOI: $10.1016 /$ j.snb.2011.05.059

[195] R. Pohle, O. von Sicard, M. Fleischer, H.-P. Frerichs, C. Wilbertz, and I. Freund, "Gate pulsed readout of floating gate FET gas sensors," Proc. Eng., vol. 5, pp. 13-16, 2010.

DOI: $10.1016 /$ j.proeng.2010.09.036

[196] Rohland Pohle, "Transient operation techniques for gas sensor applications," Proc. Eng., vol. 47, pp. 1466-1473, 2012.

DOI: $10.1016 /$ j.proeng.2012.09.432

[197] J. Wöllenstein, M. Jägle, and H. Böttner, "A gas sensitive tin oxide thin-film transistor," in Advanced gas sensing - The electroadsorptive effect and related 
techniques, Theodor Doll, Ed. Norwell, Massachusetts: Kluwer Academic Publishers, 2003, ch. 4, pp. 85-99.

ISBN: $1-4020-7433-6$

[198] W. Kim, A. Javey, O. Vermesh, Q. Wang, Y. Li, and H. Dai, "Hysteresis caused by water molecules in carbon nanotube field-effect transistors," Nano Letters, vol. 3, no. 2, pp. 193-198, 2003.

DOI: $10.1021 / \mathrm{n} 10259232$

[199] A. Robert-Peillard and S.V. Rotkin, "Modeling hysteresis phenomena in nanotube field-effect transistors," IEEE Trans. Nanotechnol., vol. 4, no. 2, pp. $284-288,2005$.

DOI: $10.1109 /$ TNANO.2004.842053

[200] T. Helbling, R. Pohle, L. Durrer, C. Stampfer, C. Roman, A. Jungen, M. Fleischer, and C. Hierold, "Sensing NO2 with individual suspended singlewalled carbon nanotubes," Sens. Actuators B, vol. 132, pp. 491-497, 2008.

DOI: $10.1016 /$ j.snb.2007.11.036

[201] M. Mattmann, T. Helbling, L. Durrer, C. Roman, R. Pohle, M. Fleischer, and C. Hierold, "Hysteresis reduction and measurement range enhancement of carbon nanotube based NO2 gas sensors by pulsed gate voltages," Proc. Chem., vol. 1, pp. 1431-1434, 2009.

DOI: $10.1016 /$ j.proche.2009.07.357

[202] M. Mattmann, D. Bechstein, C. Roman, K. Chikkadi, and C. Hierold, "Reduction of gate hysteresis above ambient temperature via ambipolar pulsed gate sweeps in carbon nanotube field effect transistors for sensor applications," App. Phys. Lett., vol. 97, p. 153103, 2010.

DOI: $10.1063 / 1.3499363$

[203] Y.W. Chang, J.S. Oh, S. H. Yoo, H.H. Choi, and K.-H. Yoo, "Electrically refreshable carbon-nanotube-based gas sensors," Nanotechnology, vol. 18, p. 435504, 2007.

DOI: $10.1088 / 0957-4484 / 18 / 43 / 435504$

[204] S. Fischer, R. Pohle, E. Magori, D. Schönauer-Kamin, M. Fleischer, and R. 
Moos, "Pulsed polarization of platinum electrodes on YSZ," Solid State Ionic, vol. 225, pp. 371-375, 2012.

DOI: $10.1016 /$ j.ssi.2012.03.020

[205] P. Reimann and A. Schütze, "Sensor arrays, virtual multisensors, data fusion, and gas sensor data evaluation," in Gas Sensing Fundamentals, D. Kohl and T. Wager, Eds.: Springer, 2014.

ISBN: 978-3-642-54518-4

[206] Markus Engel, "Untersuchungen zur Kalibrierbarkeit von Gassensoren im temperaturzyklischen Betrieb," dissertation, Saarland University, Saarbrücken, Germany, 2008, in Aktuelle Berichte, Shaker Verlag.

ISBN: 978-3832274467

[207] Stephan Horras, "Adaptierbares Messsystem zur Detektion von Gasen und Gerüchen basierend auf Halbleitergassensoren im temperaturzyklischen Betrieb," dissertation, Saarland University, Saarbrücken, Germany, 2011.

ISBN: 978-3844001013

[208] M. Engel, M. Baumbach, T. Kammerer, and A. Schütze, "Preparation of microstructured pellistors and their application for fast fuel vapor discrimination," in Proc. IEEE Intern. Conf. on MEMS, Maastricht, 2004, pp. 268-271.

DOI: 10.1109/MEMS.2004.1290574

[209] U. Weimar and W. Göpel, "A.C. measurements on tin oxide sensors to improve selectivities and sensitivities," Sens. Actuators B, vol. 26-27, pp. 13$18,1995$.

DOI: 10.1016/0925-4005(94)01547-U

[210] M. Schüler, T. Sauerwald, J. Walter, and A. Schütze, "Low-cost impedance spectroscope for metal oxide gas sensors," in IWIS - Intern. Workshop on Impedance Spectroscopy, Chemnitz, 2013.

[211] P. Reimann, A. Dausend, and A. Schütze, "A self-monitoring and selfdiagnosis strategy for semiconductor gas sensor systems," in Proc. IEEE Sensors 2008, Lecce, Italy, Oct. 27-29, 2008. 
DOI: 10.1109/ICSENS.2008.4716415

[212] M. Schüler, T. Sauerwald, and A. Schütze, "Metal oxide semiconductor gas sensor self-test using Fourier-based impedance spectroscopy," J. Sens. Sens. Syst., vol. 3, pp. 213-221, 2014.

DOI: $10.5194 /$ jsss-3-213-2014

[213] P. Reimann, M. Schüler, S. Darsch, T. Gillen, and A. Schütze, "Hardware concept and feature extraction for low-cost impedance spectroscopy for semiconductor gas sensors," in Lecture notes on impedance spectroscopy, Olaf Kanoun, Ed. London: Taylor \& Francis Group, 2012, pp. 49-61.

ISBN: 978-0-415-69838-2

[214] Svante Wold, "Chemometrics; what do we mean with it, and what do we want from it?," Chemom. Intell. Lab. Syst., vol. 30, no. 1, pp. 109-115, 1995. DOI: 10.1016/0169-7439(95)00042-9

[215] E.L. Hines, P. Boilot, J.W. Gardner, and M.A. Gongora, "Pattern analysis for electronic noses," in Handbook of Machine Olfaction, T.C. Pearce et al., Eds. Weinheim: Wiley-VCH, 2003, ch. 6, pp. 133-160.

ISBN: 3-527-30358-8

[216] Ricardo Gutierrez-Osuna, "Pattern analysis for machine olfaction," IEEE Sensors J., vol. 2, no. 3, pp. 189-202, 2002.

DOI: 10.1109/JSEN.2002.800688

[217] R.E. Shaffer, S. Rose-Pehrsson, and R.A. McGill, "A comparison study of chemical sensor array pattern recognition algorithms," Anal. Chim. Acta, vol. 384, pp. 305-317, 1999.

DOI: 10.1016/S0003-2670(98)00780-6

[218] S. Marco and A. Gutierrez-Galvez, "Signal and data processing for machine olfaction and chemical sensing: A review," IEEE Sensors J., vol. 12, no. 11, pp. 3189-3214, 2012.

DOI: 10.1109/JSEN.2012.2192920

[219] C. Bur, M. Bastuck, A. Lloyd Spetz, M. Andersson, and A. Schütze, "Selectivity enhancement of SiC-FET gas sensors by combining temperature 
and gate bias cycled operation using multivariate statistics," Sens. Actuators B, vol. 193, pp. 931-940, 2014.

DOI: $10.1016 /$ j.snb.2013.12.030

[220] A. Gramm and A. Schütze, "High performance solvent vapour identification with a two sensor array using temperature cycling and pattern classification," Sens. Actuators B, vol. 95, pp. 58-65, 2003.

DOI: $10.1016 / \mathrm{S} 0925-4005(03) 00404-0$

[221] MathWorks. Matlab Documentation, "Curve Fitting Toolbox".

Available: http://de.mathworks.com/help/curvefit/index.html Accessed: (2014, Dec. 04)

[222] MathWorks. Matlab Documentation, "Filtering and Smoothing Data". Available: $\quad$ http://de.mathworks.com/help/curvefit/smoothing-data.html Accessed: (2014, Dec. 04)

[223] Bronstein, Semendjajew, Musiol, and Mühling, Taschenbuch der Mathematik, 6th ed. Frankfurt am Main, Germany: Harri Deutsch, 2005.

ISBN: 3-8171-2006-0

[224] A. Savitzky and M.J.E. Golay, "Smoothing and differentiation of data by simplified least squares procedures," Anal. Chemistry, vol. 36, no. 8, pp. 16271639, 1964.

DOI: $10.1021 / \mathrm{ac} 60214 \mathrm{a} 047$

[225] J. Steiner, Y. Termonia, and J. Deltour, "Smoothing and differentiation of data by simplified least square procedure," Anal. Chemistry, vol. 44, no. 11, pp. 1906-1909, September 1972.

DOI: 10.1021/ac60319a045

[226] H.H. Madden, "Comments on the Savitzky-Golay convolution method for least-squares fit smoothing and differentiation of digital data," Anal. Chemistry, vol. 50, no. 9, pp. 1383-1386, August 1978.

DOI: $10.1021 / \mathrm{ac} 50031 \mathrm{a} 048$

[227] Eduard Leonhardt, "Vergleich von Multisensorsignalverarbeitungsmethoden

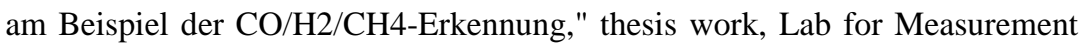


Technology, Saarland University, Saarbrücken, Germany, 2007.

[228] C. Distante, M. Leo, P. Siciliano, and K.C. Persaud, "On the study of feature extraction methods for an electronic nose," Sens. Actuators B, vol. 87, no. 2, pp. 274-288, 2002.

DOI: $10.1016 / \mathrm{S} 0925-4005(02) 00247-2$

[229] George H. Dunteman, Principal Component Analysis. Beverly Hills, USA: Sage University Paper series: quantitative applications in the social sciences, 1989.

ISBN: 978-0803931046

[230] R.O. Duda, P.E. Hart, and D.G. Stork, Pattern Classification, 2nd ed. New York, Chichester, Weinheim, Brisbane, Singapore, Toronto: John Wiley \& Sons, 2000.

ISBN: 978-0471056690

[231] R.A. Fisher, "The use of multiple measurements in taxanomic problems," Annals of Eugenics, vol. 7, no. 2, pp. 179-188, 1936.

DOI: 10.1111/j.1469-1809.1936.tb02137.x

[232] William R. Klecka, Discriminant Analysis. Beverly Hills, USA: Sage University Paper series on quantitative applications in the social sciences, No. 19, 1980.

ISBN: 0-8039-1491-1

[233] A. Heilig, N. Barsan, U. Weimar, M. Schweizer-Berberich, J.W. Gardner, and W. Göpel, "Gas identification by modulating temperatures of $\mathrm{SnO} 2$-based thick film sensors," Sens. Actuators B, vol. 43, no. 1-3, pp. 45-51, 1997. DOI: $10.1016 / \mathrm{S} 0925-4005(97) 00096-8$

[234] R. Ionescu and E. Llobet, "Wavelet transform-based fast feature extraction from temperature modulated semiconductor gas sensors," Sens. Actuators B, vol. 81, no. 2-3, pp. 289-295, 2002.

DOI: 10.1016/S0925-4005(01)00968-6

[235] Wenyi Zhao, "Discriminant analysis of principal components for face recognition," in Proc. of the third IEEE Intern. Conf. on Automatic Face and 
Gesture Recognition 1998, Nara, Japan, Apr 14-161998, pp. 336-341.

DOI: 10.1109/AFGR.1998.670971

[236] W. Zhao, A. Krishnaswamy, R. Chellappa, D.L. Swets, and J. Weng, "Discriminant Analysis of principal components for face recognition," in Face Recognition: From Theory to Applications, H. Wechsler et al., Eds. Berlin: Springer-Verlag, 1998, ch. NATO ASI Series vol. 163, pp. 73-85.

ISBN: 978-3-642-72203-5

[237] Jianke Li, "Face recognition based on PCA and LDA combination feature extraction," in 1st Intern. Conf. on Information Science and Engineering ICISE 2009, Nanjing, Japan, Dez. 26-28, 2009, pp. 1240-1243.

DOI: 10.1109/ICISE.2009.581

[238] A.G. Mignani, L. Ciaccheri, A.A. Mencaglia, B. Gordillo, M.L. GonzalezMiret, F.J. Heredia, and B. Culshaw, "Optical spectroscopy and pattern recognition techniques for discriminating and classifying Scotch whiskies," in Proc. IEEE Sensors 2011, Limerick, Ireland, Oct 28-31 2011, pp. 1946-1949.

DOI: 10.1109/ICSENS.2011.6126980

[239] L. Fernandez, A. Gutierrez-Galvez, and S. Marco, "Robustness to sensor damage of a highly redundant gas sensor array," Proc. Eng., vol. 87, pp. 851854, Sep 7-10 2014, Eurosensors XXVIII 2014.

DOI: $10.1016 /$ j.proeng.2014.11.287

[240] M. Wang, A. Perera, and R. Gutierrez-Osuna, "Principal Discriminants Analysis for small-sample-size problems: application to chemical sensing," in Proc. IEEE Sensors 2004, Vienna, Austria, Oct. 24-27, 2004.

[241] A. Vergara, E. Martinelli, R. Huerta, A. D'Amico, and C. Di Natale, "Orthogonal decomposition of chemo-sensory cues," Sens. Actuators B, vol. 159, pp. 126-134, 2011.

DOI: $10.1016 /$ j.snb.2011.06.060

[242] M.K. Muezzinoglu, A. Vergara, R. Huerta, N. Rulkov, M. Rabinovich, A. Selverston, and H.D.I. Abarbanel, "Acceleration of chemo-sensory information processing using transient features," Sens. Actuators B, vol. 137, pp. 507-512, 
2009.

DOI: $10.1016 /$ j.snb.2008.10.065

[243] A. Vergara, E. Martinelli, E. Llobet, F. Giannini, A. D'Amico, and C. Di Natale, "An alternative global feature extraction of temperature modulated micro-hotplate gas sensors array using an energy vector approach," Sens. Actuators B, vol. 124, no. 2, pp. 352-359, 2007.

DOI: $10.1016 /$ j.snb.2006.12.050

[244] R. Gutierrez-Osuna, H.T. Nagle, and S.S. Schiffman, "Transient response analysis of an electronic nose using multi-exponential models," Sens. Actuators B, vol. 61, pp. 170-182, 1999.

DOI: $10.1016 / \mathrm{S} 0925-4005(99) 00290-7$

[245] Peter Reimann, "Entwicklung von Auswertealgorithmen zur effizienten Branderkennung unter Tage," diploma thesis, Laboratory for Measurement Technology, Saarland University, Saarbrücken, Germany, 2006.

[246] C. Bur, P. Reimann, M. Andersson, A. Schütze, and A. Lloyd Spetz, "Increasing the selectivity of Pt-gate $\mathrm{SiC}$ field effect gas sensors by dynamic temperature modulation," IEEE Sensors J., vol. 12, no. 6, pp. 1906-1913, 2012 .

DOI: 10.1109/JSEN.2011.2179645

[247] N. Helwig, E. Pignanelli, and A. Schütze, "Condition monitoring of a complex hydraulic system using multivariate statistics," in Proc. International Instrumentation and Measurement Technology Conference (I2MTC ), Pisa, Italy, 2014, accepted.

[248] E.L. Hines, E. Llobet, and J.W. Gardner, "Electronic noses: a review of signal processing techniques," IEEE Proc.-Circuits Devices Syst., vol. 146, no. 6, pp. 297-310, 1999.

DOI: 10.1049/ip-cds:19990670

[249] L.A. Zadeh, "Fuzzy sets," Information and Control, vol. 8, no. 3, pp. 338-353, 1965.

DOI: 10.1016/S0019-9958(65)90241-X 
[250] K. Backhaus, B. Erichson, P. Plinke, and R. Weiber, Multivariate Analysemethoden, 13th ed. Berlin, Heidelberg, New York: Verlag, Springer, 2011.

ISBN: 978-3-642-16490-3

[251] T. Hastie, R. Tibshirani, and J. Friedman, The elements of statistical learning, 2nd ed. New York: Springer-Verlag, 2009.

ISBN: 978-0-387-84858-7

[252] Jan Mitrovics, "Linear methods in smart sensor arrays," in Lecture notes ISOCS Winter School on data analysis, robotics and mobile applications of chemical sensors, Kühtai, Austria, 2012.

[253] IBM Corporation, 2011, pp. 282-293, IBM SPSS Statistics 20 Algorithms. [Online].

ftp://public.dhe.ibm.com/software/analytics/spss/documentation/statistics/20.0/ $\underline{\text { en/client/Manuals/ }}$

[254] C.H. Park and H. Park, "A comparison of generalized linear discriminant analysis algorithms," Pattern Recognition, vol. 41, pp. 1083-1097, 2008.

DOI: 10.1016/j.patcog.2007.07.022

[255] R. Gutierrez-Osuna and H.T. Nagle, "A method for evaluating datapreprocessing techniques for odor classification with an array of gas sensors," IEEE Trans Syst Man Cybern B Cybern, vol. 29, no. 5, pp. 626 - 632, 1999. DOI: $10.1109 / 3477.790446$

[256] B. Raman, D.C. Meier, J.K. Evju, and S. Semancik, "Designing and optimizing microsensor arrays for recognizing chemical hazards," Sens. Actuators B, vol. 137, pp. 617-629, 2009.

DOI: 10.1016/j.snb.2008.11.053

[257] IBM Corporation, "Discriminant Analysis," in SPSS Statistics Base 20, 20th ed.: IBM Corporation, 2011, ch. 21, pp. 146-152.

[258] Svante Wold, "Principal Component Analysis," Chemon. Intell. Lab. Syst., vol. 2, pp. 37-52, 1987. 
DOI: $10.1016 / 0169-7439(87) 80084-9$

[259] S. Wold, M. Sjöström, and L. Eriksson, "PLS-regression: a basic tool of chemometrics," Chemom. Intell. Lab. Syst., vol. 58, pp. 109-130, 2001. DOI: 10.1016/S0169-7439(01)00155-1

[260] P. Geladi and R. Kowalski, "Partial least-squares regression: a tutorial," Anal. Chim. Act., vol. 185, pp. 1-17, 1985.

DOI: $10.1016 / 0003-2670(86) 80028-9$

[261] Sijmen de Jong, "SIMPLS: An alternative approach to partial least squares regression," Chemom. Intell. Lab. Syst., vol. 18, no. 3, pp. 251-263, 1993. DOI: 10.1016/0169-7439(93)85002-X

[262] J.P.A. Martins, R.F. Teófilo, and M.M.C. Ferreira, "Computational performance and cross-validation error precision of five PLS algorithms using designed and real data sets," J. Chemom., vol. 20, pp. 320-332, 2010.

DOI: $10.1002 / \mathrm{cem} .1309$

[263] M. Barker and W. Rayens, "Partial least squares for discrimination," $J$. Chemom., vol. 17, no. 3, pp. 166-173, 2003.

DOI: $10.1002 /$ cem.785

[264] A. Bermak, S.B. Belhouari, M. Shi, and D. Martinez, "Patter recogntion techniques for odor discrimination in gas sensor arrays," in Encyclopedia of Sensors, C.A Grimes, E.C. Dickey, and M.V. Pishko, Eds.: American Scientific Publishers, 2006, ch. 7, pp. 349-365.

ISBN: $1-58883-066-7$

[265] Ricado Gutierrez-Osuna. Lecture notes on pattern recognition, , 2014. Available: http://psi.cse.tamu.edu/teaching/lecture_notes/

[266] J.P. Marques de Sa, Pattern Recognition. Berlin, Heidelberg, Germany: Springer, 2001.

ISBN: 3-540-42297-8

[267] Michael Bahners, "Entwurf und Realisierung der Ablaufsteuerung, Datenaufnahme und -Visualisierung einer rechnergesteuerten Testanlage für 
Mikro-Gassensoren mit LabVIEW," diploma thesis, University for Applied Science Niederrhein, Krefeld, Germany, 1999.

[268] Michael Stucky, "Entwurf, Aufbau und Erprobung eines Klimaprüfstandes für Gassensoren," diploma thesis, Lab for Measurement Technology, Saarland University, Saarbrücken, Germany, 2002.

[269] Tim Ettel, "Entwurf, Aufbau und Erprobung einer hard- und softwareseitigen Prüfstandserweiterung für variable Umgebungsbedingungen," diploma thesis, Lab for Measurement Technology, Saarland University, Saarbrücken, Germany, 2003.

[270] N. Helwig, M. Schüler, C. Bur, A. Schütze , and T. Sauerwald, "Gas mixing apparatus for automated gas sensor characterization," Meas. Sci. Technol., vol. $25,2014$.

DOI: $10.1088 / 0957-0233 / 25 / 5 / 055903$

[271] Christian Bur, "Investigations on dynamic temperature modulation of Pt-gate SiC field effect gas sensors," thesis, Lab for Measurement Technology, Saarland University, Saarbrücken, Germany, 2010.

[272] M. Andersson, R. Pearce, and A. Lloyd Spetz, "New generation SiC based field effect transistor gas sensors," Sens. Actuators B, vol. 179, pp. 95-106, 2013.

DOI: 10.1016/j.snb.2012.12.059

[273] Christian Bur, "New method for selectivtiy enhancement of SiC field effect gas sensors for quantification of NOx," diploma thesis, Lab for Measurement Technology, Saarland University, Saarbrücken, Germany, 2011.

[274] MathWorks. Matlab Documentation, "Statistics Toolbox".

Available: http://de.mathworks.com/help/stats/index.html Accessed: (2014, Dec. 04)

[275] Tizian Schneider, "Klassifikation von Gassensordaten mit Support Vector Machines," bachelor thesis, Lab for Measurement Technology, Saarland University, Saarbrücken, Germany, 2013. 
[276] Manuel Bastuck, "Quantification of volatile organic compounds," master thesis, Lab for Measurement Technology, Saarland University, Saarbrücken, Germany, 2014.

[277] Mathworks. Matlab Documentation, "manova1".

Available: http://de.mathworks.com/help/stats/manova1.html Accessed: (2014, Dec. 04)

[278] MathWorks. Matlab Documentation, "pca".

Available: http://de.mathworks.com/help/stats/pca.html Accessed: (2014, Dec. 04)

[279] MathWorks. Matlab Documentation, "cvpartition".

Available: http://de.mathworks.com/help/stats/cvpartition.html Accessed: (2014, Dec. 04)

[280] MathWorks. Matlab Documentation, "datasample".

Available: http://de.mathworks.com/help/stats/datasample.html Accessed: (2014, Dec. 04)

[281] MathWorks. Matlab Documentation, "mahal".

Available: http://de.mathworks.com/help/stats/mahal.html Accessed: (2014, Dec. 04)

[282] MathWorks. Matlab Documentation, "knnsearch".

Available: http://de.mathworks.com/help/stats/knnsearch.html Accessed: (2014, Dec. 04)

[283] C. Bur, P. Reimann, M. Andersson, A. Schütze, and A. Lloyd Spetz, "Increasing the selectivity of Pt-gate $\mathrm{SiC}$ field effect gas sensors by dynamic temperature modulation," in Proc. IEEE Sensors 2010, Waikoloa, HI, USA, Nov. 1-4, 2010, pp. 1267-1272.

DOI: 10.1109/ICSENS.2010.5690598

[284] C. Bur, M. Bastuck, M. Andersson, A. Schütze, and A. Lloyd Spetz, "Influence of a changing gate bias on the sensing properties of $\mathrm{SiC}$ field effect gas sensors," in Proc. IMCS2012 - the 14th International Meeting on Chemical Sensors, Nuremberg, Germany, 2012, pp. 140-143. 
DOI: 10.5162/IMCS2012/1.5.4

[285] Manuel Bastuck, "Einfluss der Variation des Gate-Potentials auf die Sensitivität und Selektivität von GASFETs," bachelor thesis, Lab for Measurement Technology, Saarland University, Saarbrücken, Germany, 2012.

[286] M. Bastuck, C. Bur, A. Lloyd Spetz, M. Andersson, and A Schütze, "Gas identification based on bias induced hysteresis of a gas-sensitive SiC field effect transistor," J. Sens. Sens. Syst., vol. 3, pp. 9-19, 2014.

DOI: $10.5194 /$ jsss-3-9-2014

[287] S. Nakagomi, A. Fukumura, Y. Kokubun, S. Savage, H. Wingbrant, M. Andersson, I. Lundström, M. Löfdahl, and A. Lloyd Spetz, "Influence of gate bias of MISiC-FET gas sensor device on the sensing properties," Sens. Actuators B, vol. 108, pp. 501-507, 2005.

DOI: $10.1016 /$ j.snb.2004.11.057

[288] M. Bastuck, C. Bur, A. Lloyd Spetz, M. Andersson, and A. Schütze, "Identification of ammonia and carbon monoxide based on the hysteresis of a gas-sensitive SiC field effect transistor," in Proc. Transducers 2013 and Eurosensors XXVII, Barcelona, Spain, June 16-20, 2013.

DOI: $10.1109 /$ Transducers.2013.6626749

[289] C. Petit, D. Zander, K. Lmimouni, M. Ternisien, D. Tondelier, S. Lenfant, and D. Vuillaume, "Gate pulse electrical method to characterize hysteresis phenomena in organic field effect transistor," Org. Electron., vol. 9, pp. 979984, 2008.

DOI: 10.1016/j.orgel.2008.07.013

[290] K. Chatty, S. Banerjee, T.P. Chow, and R.J. Gutmann, "Hysteresis in transfer characteristics in 4H-SiC depletion/accumulation-mode MOSFETs," IEEE Electron Device Lett., vol. 23, no. 6, pp. 330-332, 2002.

DOI: $10.1109 /$ LED.2002.1004225

[291] C. Bur, M. Engel, S. Horras, and A. Schütze, "Drift compensation of virtual multisensor systems," in IMCS2014 - the 15th International Meeting on Chemical Sensors, Buenos Aires, Argentina, March 16-19, 2014. 
[292] G. Horner and C. Hierold, "Gas analysis by partial model building," Sens. Actuators B, vol. 2, pp. 173-184, 1990.

DOI: $10.1016 / 0925-4005(90) 85002-G$

[293] T. Conrad, P. Reimann, and A. Schütze, "A hierarchical strategy for underground early fire detection based on a T-cycled semiconductor gas sensor," in Proc. IEEE Sensors 2007, Atlanta, GA, USA, Oct. 28-31, 2007.

DOI: 10.1109/ICSENS.2007.4388629

[294] C. Bur, M. Andersson, A. Lloyd Spetz, and A. Schütze, "Hierarchical strategy for quantification of NOx in a varying background of typical exhaust gases," in Proc. IEEE Sensors 2011, Limerick, Ireland, Oct. 28-31, 2011, pp. 137-140. DOI: 10.1109/ICSENS.2011.6127046

[295] C. Bur, P. Reimann, A. Schütze, M. Andersson, and A. Lloyd Spetz, "New method for selectivity enhancement of $\mathrm{SiC}$ field effect gas sensors for quantification of NOx," in Proc. SPIE 8066 - Smart Sensors, Actuators, and MEMS, Prague, Czech Republic, April 18-20 2011.

DOI: $10.1117 / 12.886431$

[296] C. Bur, P. Reimann, M. Andersson, A. Lloyd Spetz, and A. Schütze, "New method for selectivity enhancement of $\mathrm{SiC}$ field effect gas sensors for quantification of NOx," Microsystem Technologies, vol. 18, no. 7-8, pp. 10151025, 2012.

DOI: $10.1007 / \mathrm{s} 00542-012-1434-\mathrm{z}$

[297] C. Bur, N. Helwig, A. Schütze, A. Lloyd Spetz, and M. Andersson, "Detecting volatile organic compounds in the ppb range with platinum-gate SiC-field effect transistors," in Proc. IEEE Sensors 2013, Balitmore, MD, USA, Nov. 36, 2013, pp. 617-620.

DOI: $10.1109 /$ ICSENS.2013.6688279

[298] C. Bur, M. Bastuck, D. Puglisi, A. Schütze, A. Lloyd Spetz, and M. Andersson, "Discrimination and quantification of volatile organic compounds in the ppb range with gas sensitive SiC-field effect transistors," Proc. Eng., vol. 87, pp. 604-607, Sep 7-10 2014, Eurosensors XXVIII. 
DOI: 10.1016/j.proeng.2014.11.561

[299] C. Bur, M. Bastuck, D. Puglisi, A. Schütze, A. Lloyd Spetz, and M. Andersson, "Discrimination and quantification of volatile organic compounds in the ppb-range with gas sensitive SiC-FETs using multivariate statistics," Sens. Actuators B, 2014, submitted.

[300] D. Puglisi, J. Eriksson, C. Bur, A. Schütze, A. Lloyd Spetz, and M. Andersson, "Silicon-carbide field effect transistors for dection of ultra-low concentrations of hazardous volatile organic compounds," Mater. Sci. Forum, vol. 779-780, pp. 1067-1070, 2014.

DOI: $10.4028 /$ www.scientific.net/MSF.778-780.1067

[301] C. Bur, M. Bastuck, M. Andersson, A. Lloyd Spetz, and A. Schütze, "Combination of temperature cycled and gate bias cycled operation to enhance the selectivity of SiC-FET gas sensors," in Proc. Transducers 2013 and Eurosensors XXVII, Barcelona, Spain, June 16-20, 2013, pp. 2041-2044.

DOI: $10.1109 /$ Transducers.2013.6627199

[302] D.A. Burgard, G.A. Bishop, D.H. Stedman, V.H. Gessner, and C. Daeschlein, "Remote sensing of in-use heavy-duty diesel trucks," Environ. Sci. Technol., vol. 40, no. 22, pp. 6938-6942, 2006.

DOI: 10.1021/es060989a 



\section{Own Publications}

The following articles have been published during my $\mathrm{PhD}$ studies. All necessary permissions for potential reuse of the material in the present thesis have been obtained from the publishers.

\section{Peer-Reviewed Journal Articles}

J1 C. Bur, P. Reimann, M. Andersson, A. Lloyd Spetz, and A. Schütze, "Increasing the selectivity of Pt-gate SiC field effect gas sensors by dynamic temperature modulation," IEEE Sensors J., vol. 12, no. 6, pp. 1906-1913, 2012.

DOI: 10.1109/JSEN.2011.2179645

J2 C. Bur, P. Reimann, M. Andersson, A. Lloyd Spetz, and A. Schütze, "New method for selectivity enhancement of $\mathrm{SiC}$ field effect gas sensors for quantification of $\mathrm{NO}_{\mathrm{x}}$, " Microsystem Techn., vol. 18, iss. 7-8, pp. 1015-1025, 2012.

DOI: $10.1007 / \mathrm{s} 00542-012-1434-\mathrm{z}$

J3 A. Lloyd Spetz, J. Huotari, C. Bur, R. Bjorklund, J. Lappalainen, H. Jantunen, A. Schütze, and M. Andersson, "Chemical sensor systems for emission control from combustions," Sens. Actuators B, vol. 187, pp. 184-190, 2013. DOI: $10.1016 /$ j.snb.2012.10.078

J4 A. Lloyd Spetz, Z. Darmastuti, R. Bjorklund, M. Andersson, C. Bur, A. Schütze, J. Huotari, J. Lappalainen, H. Jantunen, and N. Lindqvist, "Improved chemical sensors track and control emissions," SPIE Newsroom, May 1, 2013.

DOI: $10.1117 / 2.1201304 .004839$ 
J5 Z. Darmastuti，C. Bur，P. Möller， R. Rahlin， N. Lindqvist， M. Andersson, A. Schütze, and A. Lloyd Spetz, "SiC - FET based $\mathrm{SO}_{2}$ sensor for power plant emission applications,“ Sens. Actuators B, vol. 194, pp. 511-520, 2014. DOI: $10.1016 /$ j.snb.2013.11.089

J6

D. Puglisi, J. Eriksson, C. Bur, A. Schütze, A. Lloyd Spetz, and M. Andersson, "Silicon carbide field effect transistors for detection of ultralow concentrations of hazardous volatile organic compounds," Mat. Sci. Forum, vol. 779-780, pp. 1067-1070, 2014.

DOI: 10.4028/www.scientific.net/MSF.778-780.1067

J7 C. Bur, M. Bastuck, A. Lloyd Spetz, M. Andersson, and A. Schütze, "Selectivity enhancement of SiC-FET gas sensors by combining temperature and gate bias cycled operation using multivariate statistics," Sens. Actuators B, vol. 193, pp. 931-940, 2014.

DOI: $10.1016 /$ j.snb.2013.12.030

J8 M. Bastuck, C. Bur, A. Lloyd Spetz, M. Andersson, and A. Schütze, "Gas identification based on bias induced hysteresis of a gas-sensitive SiC field effect transistor,” J. Sens. Sens. Syst. vol. 3, pp. 9-19, 2014.

DOI: $10.5194 /$ jsss-3-9-2014

J9 N. Helwig, M. Schüler, C. Bur, A. Schütze, and T. Sauerwald, "Gas mixing apparatus for automated gas sensor characterization," Meas. Sci. Technol., vol. 25 , no. $5,2014$.

DOI:10.1088/0957-0233/25/5/055903

J10 C. Bur, M. Andersson, A. Lloyd Spetz, and A. Schütze, "Detecting volatile organic compounds in the ppb rang with gas sensitive platinum gate SiC-field effect transistors," IEEE Sensors J., vol. 14, no. 9, pp. 3221-3228, 2014.

DOI: 10.1109/JSEN.2014.2326693

J11 Z. Darmastuti, C. Bur, N. Lindqvist, M. Andersson, A. Schütze, and A. Lloyd Spetz, "Hierarchical methods to improve the performance of the SiC FET as $\mathrm{SO}_{2}$ sensor in flue gas desulphurization systems," Sens. Actuators B, vol. 206, pp. 609-616, 2015.

DOI: $10.1016 /$ j.snb.2014.09.113 
J12 C. Bur, M. Bastuck, A. Schütze, J. Juuti, A. Lloyd Spetz, and M. Andersson, "Characterization of ash particles with a micro heater and gas sensitive $\mathrm{SiC}$ field effect transistors," J. Sens. Sens. Syst., 3, 305-313, 2014.

DOI: $10.5194 /$ jsss-3-305-2014

J13 D. Puglisi, J. Eriksson, C. Bur, A. Schütze, A. Lloyd Spetz, and M. Andersson, "Catalytic metal-gate field effect transistors based on SiC for indoor air quality control,“ J. Sens. Sens. Syst., vol. 4, pp. 1-8, 2015.

DOI: $10.5194 /$ jsss-4-1-2015

J14 C. Bur, M. Bastuck, D. Puglisi, A. Schütze, A. Lloyd Spetz, and M. Andersson, "Discrimination and quantification of volatile organic compounds in the ppb-range with gas sensitive SiC-FETs using multivariate statistics," Sens. Actuators B, submitted 


\section{My Contribution to These Journal Articles}

J1 I performed all the planning and experimental work, including data evaluation as well as the writing. The sensors were developed and processed at Linköping University, and the sensor hardware was supplied by $3 \mathrm{~S} \mathrm{GmbH}$ Sensors, Signal Processing, Systems, Saarbrücken, Germany.

J2 I performed all the planning and experimental work, including data evaluation as well as the writing. The sensors were developed and processed at Linköping University, and the sensor hardware was supplied by $3 S \mathrm{GmbH}$ Sensors, Signal Processing, Systems, Saarbrücken, Germany.

J3 I performed the planning and the measurements concerning particle detection using the example of fly ash samples on a micro heater as well as the corresponding data evaluation.

J4 I contributed the work concerning particle detection using the example of fly ash samples on a micro heater.

J5 I helped by the definition of the sensor operating mode and the evaluation by multivariate statistics.

J6 I helped D. Puglisi by setting up and planning the experiments.

J7 I performed all the planning and experimental work, including data evaluation as well as the writing. The sensors were developed and processed by SenSiC AB, Kista, Sweden and provided by Linköping University, Sweden. The sensor hardware was supplied by $3 S \mathrm{GmbH}$ - Sensors, Signal Processing, Systems, Saarbrücken, Germany.

J8 I supervised M. Bastuck who did the work as part of his bachelor thesis. I was involved in the planning of the experiments and the discussion of the results.

J9 I was involved in the planning and design of the gas mixing system and actively participated in all relevant meetings. I did the measurements with SiC-FETs with the new gas mixing system. 
J10 I performed all the planning and experimental work, including literature review, data evaluation as well as the writing. The sensors were developed and processed by SenSiC AB, Kista, Sweden and provided by Linköping University, Sweden. The sensor hardware was supplied by $3 \mathrm{~S} \mathrm{GmbH} \mathrm{-}$ Sensors, Signal Processing, Systems, Saarbrücken, Germany.

J11 I helped by the definition of the sensor operating mode and the evaluation by multivariate statistics.

J12 I performed all the planning and experimental work, including data evaluation as well as the writing. The sensors were developed and processed by $\mathrm{SenSiC}$ AB, Kista, Sweden and provided by Linköping University, Sweden. The sensor hardware was supplied by $3 S \mathrm{GmbH}$ - Sensors, Signal Processing, Systems, Saarbrücken, Germany.

J13 I helped D. Puglisi by setting up and planning the experiments.

J14 I performed all the planning and experimental work, including data evaluation as well as the writing. The sensors were developed and processed by $\mathrm{SenSiC}$ $\mathrm{AB}$, Kista, Sweden and provided by Linköping University, Sweden. The sensor hardware was supplied by $3 S \mathrm{GmbH}$ - Sensors, Signal Processing, Systems, Saarbrücken, Germany. 


\section{Conference Contributions}

Contributions marked with * have been presented by myself.

C1* C. Bur, P. Reimann, M. Andersson, A. Lloyd Spetz, and A. Schütze, "Increasing the selectivity of Pt-gate $\mathrm{SiC}$ field effect gas sensors by dynamic temperature modulation," Proc. IEEE Sensors 2010, Waikoloa, HI, USA Nov. 1-4, pp. 1267-1272, 2010.

DOI: 10.1109/ICSENS.2010.5690598

C2* C. Bur, E. Pignanelli, M. Barra, and E. Tesfagiorgis, "Music Glove - A novel, versatile human machine interface device for hand- and finger movement detection based on accelerometers and MR-sensors," Proc. 11th Symposium Magnetoresistive Sensors and Magnetic Systems, Wetzlar, Germany, March 29-30, 2011.

C3 C. Bur, P. Reimann, M. Andersson, A. Lloyd Spetz, and A. Schütze, "Temperature cycled operation of $\mathrm{SiC}$ field effect gas sensors: increasing the selectivity for improved sensor systems,“ ISOEN - International Symposium on Olfaction and Electronic Nose 2011, New York, USA, May 02-05, AIP Conf. Proc. 1362, pp. 91-92, 2011.

DOI:10.1063/1.3626317

C4* C. Bur, P. Reimann, A. Schütze, M. Andersson, and A. Lloyd Spetz, "New method for selectivity enhancement of SiC field effect gas sensors for quantification of $\mathrm{NO}_{\mathrm{x}}, "$ Smart Sensors, Actuators, and MEMS, Prague, Czech Rep., April 18-20, Proc. SPIE 8066, 2011.

DOI:10.1117/12.886431

C5* C. Bur, M. Andersson, A. Lloyd Spetz, and A. Schütze, "Hierarchical strategy for quantification of $\mathrm{NO}_{\mathrm{x}}$ in a varying background of typical exhaust gases," Proc. IEEE Sensors 2011, Limerick, Ireland, Oct. 28-31, pp. 137-140, 2011. DOI: 10.1109/ICSENS.2011.6127046

C6* C. Bur, M. Bastuck, M. Andersson, A. Lloyd Spetz, and A. Schütze, "Influence of a changing gate bias on the sensing properties of $\mathrm{SiC}$ field 
effect gas sensors," Proc. IMCS 2012 the 14th International Meeting on Chemical Sensors, Nuremberg, Germany, May 20-23, pp. 140-143, 2012.

DOI: 10.5162/IMCS2012/1.5.4

C7 A. Lloyd Spetz, J. Huotari, C. Bur, J. Lappalainen, A. Schütze, and M. Andersson, "Chemical sensor systems for emission control from combustions," Proc. IMCS 2012, Nuremberg, Germany, May 20-23, pp. 635638, 2012.

DOI: $10.5162 / \mathrm{IMCS} 2012 / 7.4 .2$

C8 A. Lloyd Spetz, Z. Darmastuti, C. Bur, J. Huotari, R. Bjorklund, N. Lindquist, J. Lappalainen, H. Jantunen, A. Schütze, and M. Andersson, "Chemical sensor systems for environmental and emission control," Proc. SPIE 8725, Micro- and Nanotechnology Sensors, Systems, and Applications V, Baltimore, USA, April 29-May 2, 2013.

DOI: $10.1117 / 12.2016795$

C9* C. Bur, M. Bastuck, M. Andersson, A. Lloyd Spetz, and A. Schütze, "Combination of temperature cycled and gate bias cycled operation to enhance the selectivity of SiC-FET gas sensors, “ Proc. Transducers 2013 and Eurosensors XXVII, Barcelona, Spain, June 16-20, pp.2041-2044, 2013.

DOI: 10.1109/Transducers.2013.6627199

Nomination for outstanding poster presentation award

C10 M. Bastuck, C. Bur, A. Lloyd Spetz, M. Andersson, and A. Schütze, "Identification of ammonia and carbon monoxide based on the hysteresis of a gas-sensitive SiC field effect transistor," Proc. Transducers 2013 and Eurosensors XXVII, Barcelona, Spain, June 16-20, pp. 250-253, 2013.

DOI: $10.1109 /$ Transducers.2013.6626749

C11 Z. Darmastuti, C. Bur, P. Möller, N. Lindqvist, M. Andersson, A. Schütze, and A. Lloyd Spetz, "SiC - FET based $\mathrm{SO}_{2}$ sensor for power plant emission applications," Proc. Transducers 2013 and Eurosensors XXVII, Barcelona, Spain, June 16-20, pp. 1150-1153, 2013.

DOI: 10.1109/Transducers.2013.6626976

C12* C. Bur, A. Schütze, J. Eriksson, M. Andersson, A. Lloyd Spetz, and D. Puglisi, "Detection of low concentrations of volatile organic compounds 
with SiC-Field Effect Transistors,“ 1st Intern. Workshop of COST Action TD1105 "EuNetAir", Open Satellite Workshop inside Transducers 2013, Barcelona Spain, June 20, 2013.

C13* A. Lloyd Spetz, C. Bur, J. Houtari, Z. Darmastuti, J. Lappalainen, H. Jantunen, R. Bjorklund, N. Lindqvist, A. Schütze, and M. Andersson, "Emission monitoring and control by gas and particle sensors for clean environment," 1st Intern. Workshop of COST Action TD1105 "EuNetAir", Open Satellite Workshop inside Transducers 2013, Barcelona Spain, June 20, 2013.

C14 Z. Darmastuti, P. Möller, C. Bur, R. Rahlin, N. Lindqvist, M. Andersson, A. Schütze, and A. Lloyd Spetz, "SiC-FET based $\mathrm{SO}_{2}$ sensors for desulphurization units in power generation applications," Proc. ISOEN International Symposium on Olfaction and Electronic Nose 2013, Daegu, South Korea, July 2-5, 2013.

C15* C. Bur, N. Helwig, A. Schütze, A. Lloyd Spetz, and M. Andersson, "Detecting volatile organic compounds in the ppb range with platinum-gate SiC-field effect transistors," Proc. IEEE Sensors 2013, Baltimore, MD, USA, Nov. 3-6, pp. 617-620, 2013.

DOI: 10.1109/ICSENS.2013.6688279

C16 D. Puglisi, J. Eriksson, C. Bur, A. Schütze, A. Lloyd Spetz, and M. Andersson, "Silicon carbide field effect transistors for detection of ultralow concentrations of hazardous volatile organic compounds," ICSCRM The International Conference on Silicon Carbide and Related Materials 2013, Sept. 29 - Oct. 4, Miyazaki, Japan, 2013.

C17 C. Bur, D. Puglisi, J. Eriksson, M. Andersson, A. Lloyd Spetz, and A. Schütze, "Detection of low concentrations of volatile organic compounds with SiC-Field Effect Transistors," poster presentation at the COST Action TD1105 "EuNetAir" WG Meeting, European Environment Agency (EEA), Copenhagen, Denmark, Oct. 3-4, 2013.

C18 Z. Darmastuti，P. Möller，C. Bur， R. Rahlin， N. Lindqvist， M. Andersson, A. Schütze, and A. Lloyd Spetz, "SiC-FET based $\mathrm{SO}_{2}$ sensors for power plant applications," oral presentation at the COST Action TD1105 "EuNetAir" WG 
and MC Meeting, University of Cambridge, Queens' College, Cambridge, United Kingdome, Dez. 18-20, 2013.

C19 C. Bur, M. Engle, S. Horras, and A. Schütze, "Drift compensation of virtual multi-sensor systems based on extended calibration," IMCS 2014 - the 15th International Meeting on Chemical Sensors; Buenos Aires, Argentina, March 16-19, 2014.

C20 D. Puglisi, C. Bur, Y. H. Kang, R. Yakimova, A. Lloyd Spetz, A. Schütze, M. Andersson, and J. Eriksson, "SiC-FET and graphene-based gas sensors for sensitive detection of toxic substances in indoor environments, “IMCS 2014 the 15th International Meeting on Chemical Sensors; Buenos Aires, Argentina, March 16-19, 2014.

C21 C. Bur, M. Andersson, A. Schütze, and A. Lloyd Spetz, "The influence of gate bias on the $\mathrm{CO}$ sensing characteristics of SiC based field effect sensors," IMCS 2014 - the 15th International Meeting on Chemical Sensors; Buenos Aires, Argentina, March 16-19, 2014.

C22 J. Eriksson, D. Puglisi, C. Bur, M. Andersson, A. Lloyd Spetz, and A. Schütze, "Gas sensing with epitaxial graphene on silicon carbide: Performance tuning for air quality," 2nd Intern. Workshop of COST Action TD1105 "EuNetAir", Brindisi, Italy, March 25 - 26, 2014.

C23 D. Puglisi, C. Bur, J. Eriksson, M. Andersson, A. Lloyd Spetz, and A. Schütze, "Detection of low concentrations of volatile organic compounds with SiC-Field Effect Transistors," 2nd Intern. Workshop of COST Action TD1105 "EuNetAir", Brindisi, Italy, March 25 - 26, 2014.

C24 C. Bur, M. Bastuck, A. Schütze, A. Lloyd Spetz, J. Juuti, and M. Andersson, "Characterization of dust and ash particles with a micro heater and gas sensitive SiC field effect transistors," E-MRS Spring Meeting 2014, Lille, France, May 26-30, 2014.

C25 C. Bur, M. Bastuck, A. Schütze, A. Lloyd Spetz, and M. Andersson, "Increasing the selectivity of gas sensitive field effect transistors by dynamic operation,” E-MRS Spring Meeting 2014, Lille, France, May 26-30, 2014. 
C26 D. Puglisi, J. Eriksson, C. Bur, A. Schütze, A. Lloyd Spetz, and M. Andersson, "Catalytic metal-gate field effect transistors based on SiC for indoor air quality control," E-MRS Spring Meeting 2014, Lille, France, May 26-30, 2014.

C27* C. Bur, M. Bastuck, D. Puglisi, M. Andersson, A. Lloyd Spetz, and A. Schütze, "Discrimination and quantification of volatile organic compounds in the ppb-range with gas sensitive SiC-Field Effect Transistors," Eurosensors 2014, Brescia, Italy, Sep. 7-10, 2014.

Proc. Eng. vol. 87, pp. 604-607, 2014.

DOI: 10.1016/j.proeng.2014.11.561

C28 D. Puglisi, J. Eriksson, C. Bur, A. Schütze, A. Lloyd Spetz, and M. Andersson, "Silicon carbide field effect transistors for detection of ultralow concentrations of hazardous volatile organic compounds," AFM2014 Conference on Advanced Functional Materials, Vildmarkshotellet Kolmården, Norrköping, Sweden, Aug. 20-21, 2014.

C29 D. Puglisi, C. Bur, M. Bastuck, A. Schütze, M. Andersson, R. Yakimova, A. Lloyd Spetz, and J. Eriksson, "Mastering VOC detection for better indoor air quality," invited talk at the COST Action TD1105 "EuNetAir" WG and MC Meeting, GEBZE Institute of Technology and Bahcesehir University, Istanbul, Turkey, Dez. 3-5, 2014.

C30 C. Bur, M. Bastuck, A. Schütze, J. Juuti, M. Andersson, and A. Lloyd Spetz, "Characterization of dust and ash particles with a micro heater and gas sensitive SiC field effect transistors," invited talk at the COST Action TD1105 "EuNetAir" WG and MC Meeting, GEBZE Institute of Technology and Bahcesehir University, Istanbul, Turkey, Dez. 3-5, 2014.

C31 A. Lloyd Spetz, C. Bur, M. Sobocinski, H. Jantunen, J. Juuti, A. Schütze, and M. Andersson, "New method for control of nanoparticles in indoor environments," invited talk at the COST Action TD1105 "EuNetAir" WG and MC Meeting, GEBZE Institute of Technology and Bahcesehir University, Istanbul, Turkey, Dez. 3-5, 2014.

C32 M. Bastuck, C. Bur, T. Sauerwald, A. Lloyd Spetz, M. Andersson, and A. Schütze, "Quantification of volatile organic compounds in the ppb-range 
using Partial Least Squares Regression," AMA Congress SENSOR 2015, Nuremberg, Germany. accepted 



\section{Abbreviations and Symbols}

\section{Abbreviations}

\begin{tabular}{ll}
\hline Abbr. & Description \\
\hline ADC & Analog Digital Converter \\
ANN & Artificial Neural Networks \\
ART & Adaptive Resonance Theory \\
BAW & Bulk Acoustic Wave \\
BET & Brunauer, Emmett, and Teller isotherm \\
CA & Clustering Algorithms \\
CAQ & Cabin Air Quality \\
CC-FET & Capacitance Coupled Field Effect Transistor \\
CMOS & Complementary Metal Oxide Semiconductor \\
CNT & Carbon Nano-Tube \\
CP & Conducting Polymer \\
CV & Cross-Validation \\
DAC & Digital Analog Converter \\
DF & Discriminant Function \\
DFA & Discriminant Function Analysis \\
DL & Dipole Layer \\
DPF & Diesel Particle Filter \\
DRIFT & Diffuse Reflectance Infrared Fourier Transform Spectroscopy \\
DWT & Discrete Wavelet Transformation \\
EC & Electrochemical Cell \\
EEA & European Environment Agency \\
\hline
\end{tabular}




\begin{tabular}{|c|c|}
\hline EMA & Exponential Moving Average \\
\hline $\mathbf{E N}$ & Electronic Nose \\
\hline $\mathbf{E V}$ & Energy Vector \\
\hline FCM & Fuzzy c-Means \\
\hline FE & Field Effect (device related) \\
\hline FE & Feature Extraction (signal processing related) \\
\hline FET & Field Effect Transistor \\
\hline FFT & Fast Fourier Transformation \\
\hline FG-FET & Floating Gate Field Effect Transistor \\
\hline FIS & Fuzzy Interference Quantification \\
\hline FNN & Fuzzy Neural Network \\
\hline FTIR & Fourier Transformation InfraRed spectroscopy \\
\hline GA & Generic Algorithms \\
\hline GBCO & Gate Bias Cycled Operation \\
\hline GC-MS & Gas Chromatography - Mass Spectroscopy \\
\hline GUI & Graphical User Interface \\
\hline IAQ & Indoor Air Quality \\
\hline IAQG & Indoor Air Quality Guidelines \\
\hline IR & InfraRed \\
\hline ISFET & Ion Sensitive Field Effect Transistor \\
\hline IUPAC & International Union of Pure Applied Chemistry \\
\hline IV-curve & Current-voltage curve \\
\hline JFET & Junction Field Effect Transistor \\
\hline kNN & k-Nearest Neighbor classifier \\
\hline LDA & Linear Discriminant Analysis \\
\hline LMT & Lab for Measurement Technology \\
\hline LOOCV & Leave-One-Out Cross-Validation \\
\hline LVQ & Learning Vector Quantification \\
\hline MESFET & Metal Semiconductor Field Effect Transistor \\
\hline MFC & Mass Flow Controller \\
\hline MIS & Metal Insulator Semiconductor \\
\hline MISFET & Metal Insulator Semiconductor Field Effect Transistor \\
\hline
\end{tabular}




\begin{tabular}{|c|c|}
\hline MLP & Multi-Layer Perceptron \\
\hline MLR & Multiple Linear Regression \\
\hline MOS & Metal Oxide Semiconductor with respect to silicon \\
\hline MOX & Metal Oxide Semiconductor, for gas sensors MOX refers to a resistive type \\
\hline MSR & Mean Squared Error \\
\hline $\mathbf{m v}$ & Mean value, a kind of feature \\
\hline MWNT & Multi-Wall Nano-Tube \\
\hline Nano-FET & Nano-structured - Field Effect Transistor \\
\hline NDIR & Non-Dispersiv InfraRed \\
\hline NFS & Neuro Fuzzy Systems \\
\hline OLS & Ordinary Least Squares \\
\hline PC & Principal Component \\
\hline PCA & Principal Component Analysis \\
\hline PCR & Principal Component Regression \\
\hline PDA & Principal Discriminants Analysis \\
\hline PLS-DA & Partial Least Squares - Discriminant Analysis \\
\hline PLSR & Partial Least Squares Regression \\
\hline PNN & Probabilistic Neural Network \\
\hline ppb & Parts-per-billion \\
\hline ppm & Parts-per-million \\
\hline QMB & Quartz-crystal Micro Balance \\
\hline r.h. & Relative humidity \\
\hline RBF & Radial Basis Function \\
\hline SAW & Surface Acoustic Waves \\
\hline SCR & Selective Catalytic Reduction \\
\hline sec & Secant, i.e., slope, a kind of feature \\
\hline SEM & Scanning Electron Spectroscopy \\
\hline SG-FET & Suspended Gate Field Effect Transistor \\
\hline SG-filter & Savitzky-Golay convolution filter \\
\hline SNR & Signal-to-Noise Ratio \\
\hline SOM & Self-Organizing Maps \\
\hline SS & Surface States \\
\hline
\end{tabular}




\begin{tabular}{ll}
\hline SubVI & Sub-routine Virtual Instrument \\
SVM & Support Vector Machines \\
SVR & Support Vector Regression \\
SWNT & Single-Wall Nano-Tube \\
TCO & Temperature Cycled Operation \\
TEM & Transmission Electron Microscopy \\
TPS & Temperature Programmed Sensing \\
VFB & Flat-Band Voltage \\
VI & Virtual Instrument \\
VOC & Volatile Organic Compounds \\
Vth & Threshhold Voltage \\
YSZ & Yttria-Stabilized Zirconia \\
\hline
\end{tabular}




\section{Sensor Device Related Symbols}

\begin{tabular}{|c|c|}
\hline Symbol & Description \\
\hline$C_{\mathrm{ox}}^{\prime}$ & Capacitance per unit area \\
\hline$C_{A}$ & Capacitance of the air gap \\
\hline$C_{D R}$ & Capacitance of the depletion region \\
\hline$C_{I}$ & Insulator capacitance \\
\hline$C_{e f f}$ & Effective capacitance \\
\hline$C_{o x}$ & Capacitance of the oxide, i.e., insulator \\
\hline$E_{g}$ & Energy band gap \\
\hline$I_{D}$ & Drain current \\
\hline$N_{A}$ & Acceptor concentration \\
\hline$Q_{M}$ & Charge on the metal surface \\
\hline$Q_{s}$ & Total semiconductor charge \\
\hline$Q_{f}$ & Fixed oxide charge \\
\hline$Q_{i t}$ & Interface trapped charge \\
\hline$Q_{m}$ & Mobile ionic charge \\
\hline$Q_{n}^{\prime}$ & Charge per unit area \\
\hline$Q_{o t}$ & Oxide trapped charge \\
\hline$V_{A}$ & Early voltage \\
\hline$V_{B S}$ & Substrate bias \\
\hline$V_{D S}$ & Drain - source voltage \\
\hline$V_{F B}$ & Flat-band voltage \\
\hline$V_{G S}$ & Gate - source voltage \\
\hline$V_{o x}$ & Voltage drop over oxide, i.e., insulator \\
\hline$V_{t h}$ & Threshold voltage \\
\hline $\boldsymbol{W}^{\prime}$ & Width of the surface depletion region \\
\hline$W_{\max }^{\prime}$ & Maximum width of the surface depletion region \\
\hline$d_{o x}$ & Thickness of the oxide, i.e., insulator \\
\hline$g_{d}$ & Channel conductance \\
\hline
\end{tabular}




\begin{tabular}{|c|c|}
\hline$g_{m}$ & Transconductance \\
\hline $\boldsymbol{k}_{\text {ads }}$ & Adsorption coefficient \\
\hline $\boldsymbol{k}_{\text {des }}$ & Desorption coefficient \\
\hline$\varepsilon_{S}$ & Permittivity of the semiconductor \\
\hline$\varepsilon_{o x}$ & Permittivity of the oxide, i.e., insulator \\
\hline$\mu_{n}$ & Mobility of the charge carriers \\
\hline$\psi_{B}$ & $\begin{array}{l}\text { Potential difference between Fermi-level and intrinsic Fermi-level, also known as } \\
\text { build-in potential }\end{array}$ \\
\hline$\psi_{S}$ & Surface potential \\
\hline$\phi_{m}$ & Metal work function \\
\hline$\phi_{m s}$ & Metal-semiconductor work function difference \\
\hline$\phi_{s}$ & Semiconductor work function \\
\hline $\mathbf{W}$ & Channel width \\
\hline G & Gibb's free energy \\
\hline $\boldsymbol{H}$ & Enthalpy \\
\hline $\boldsymbol{K}$ & Transconductance coefficient \\
\hline $\boldsymbol{L}$ & Length of the channel \\
\hline$L^{\prime}$ & Reduced channel length \\
\hline$S$ & Entropy \\
\hline$T$ & Temperature \\
\hline$c$ & Concentration \\
\hline$f(\theta)$ & Adsorption probability \\
\hline$p$ & Pressure \\
\hline$q$ & Elementary charge \\
\hline$\gamma$ & Body effect coefficient \\
\hline $\boldsymbol{\theta}$ & Adsorption coverage \\
\hline$\lambda$ & Channel length modulation parameter \\
\hline$\chi$ & Semiconductor electron affinity \\
\hline
\end{tabular}




\section{Signal Processing Related Symbols}

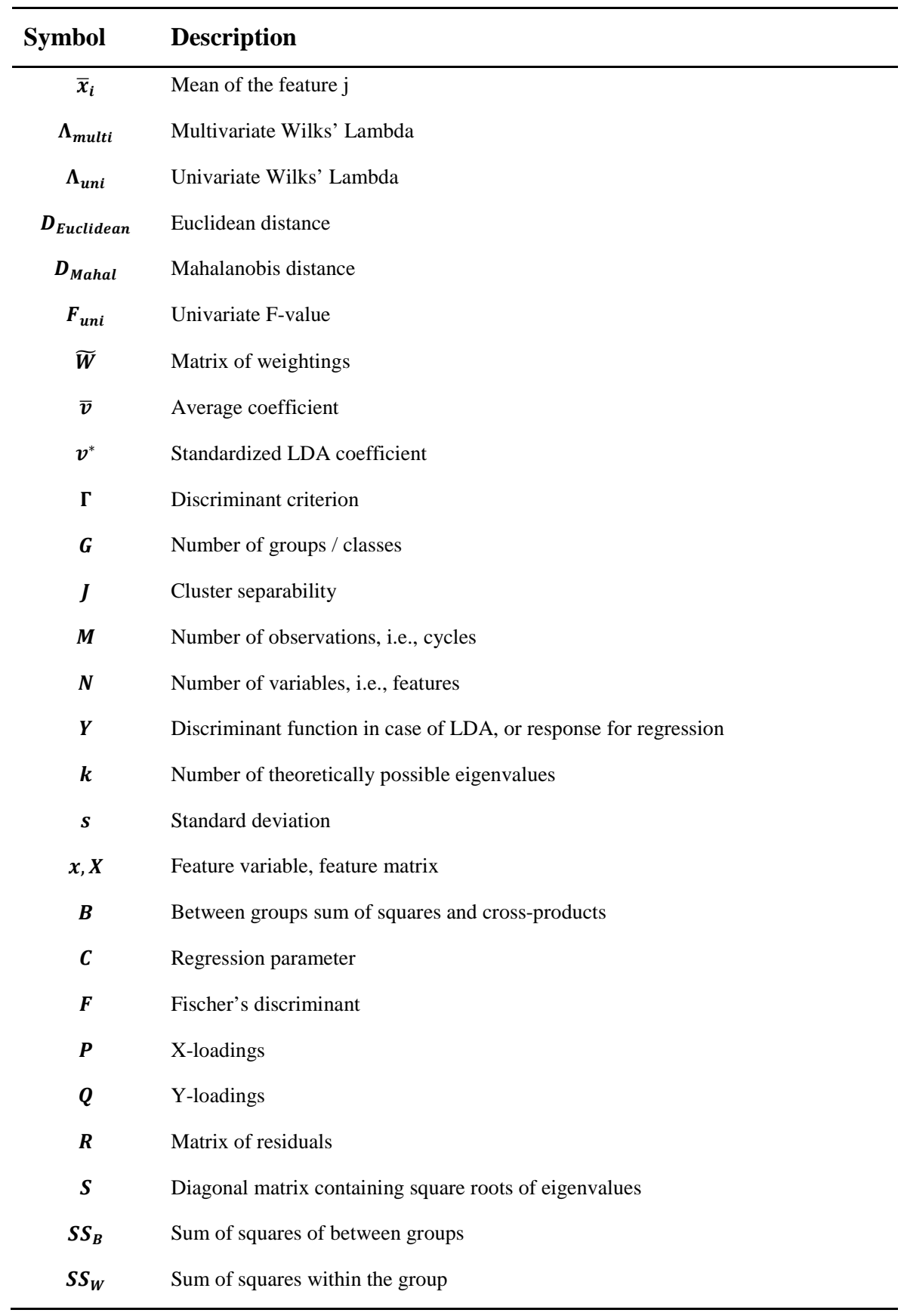


$\boldsymbol{T} \quad$ Total sum of squares and cross-products

U Matrix of eigenvectors of $X \cdot X^{T}$

$\boldsymbol{V} \quad$ Matrix containing eigenvectors, i.e., loadings

$\boldsymbol{W} \quad$ Within-groups sum of squares and cross-products

cov Variance-covariance matrix

$\boldsymbol{t}, \widetilde{\boldsymbol{T}} \quad$ Scores, scores matrix

$\boldsymbol{u} \quad$ Raw LDA coefficients

$\lambda \quad$ Eigenvalue 


\section{Chemical Symbols}

\begin{tabular}{|c|c|}
\hline Abbr. & Description \\
\hline AIN & Aluminum nitride \\
\hline $\mathrm{C}_{2} \mathrm{H}_{4}$ & Ethene \\
\hline $\mathrm{C}_{3} \mathrm{H}_{6}$ & Propene \\
\hline CNT & Carbon Nano-Tube \\
\hline CO & Carbon monoxide \\
\hline $\mathbf{C P}$ & Conducting Polymer \\
\hline GaN & Gallium nitride \\
\hline $\mathbf{H}_{2}$ & Molecular hydrogen \\
\hline $\mathrm{HC}$ & Hydrocarbons \\
\hline Ir & Iridium \\
\hline MgO & Magnesium oxide \\
\hline MWNT & Multi-Wall Nano-Tube \\
\hline $\mathbf{N H}_{3}$ & Ammonia \\
\hline NO & Nitrogen monoxide \\
\hline $\mathrm{NO}_{2}$ & Nitrogen dioxide \\
\hline $\mathrm{NO}_{\mathbf{x}}$ & Mixture of nitrogen monoxide and nitrogen dioxide, here with a ratio 10:1 \\
\hline $\mathbf{O}_{2}$ & Molecular oxygen \\
\hline $\mathbf{O H}$ & Hydroxyl group \\
\hline Pd & Palladium \\
\hline $\mathbf{P t}$ & Platinum \\
\hline $\mathbf{S i}$ & Silicon \\
\hline $\mathrm{SiC}$ & Silicon Carbide \\
\hline $\mathrm{SiO}_{2}$ & Silica, silicon dioxide \\
\hline SWNT & Single-Wall Nano-Tube \\
\hline $\mathrm{ZnO}$ & Zink Oxide \\
\hline
\end{tabular}





\section{Figures}

Fig. 2.1 Structural formula of formaldehyde, benzene, and naphthalene.

Fig. 3.1 (a) Ideal p-type MIS structure. (b) Ideal energy band diagram of an ideal MIS structure under flat band condition. Reproduced from [65] with kind permission from Wiley.

Fig. 3.2 Energy band diagram of an ideal MIS structure under certain bias. (a) When a negative bias, i.e., $\mathrm{V}<0$, (b) when a positive bias, i.e., $\mathrm{V}>0$, and (c) when a large positive bias, i.e., $\mathrm{V}>>0$ is applied. Reproduced from [65] with kind permission from Wiley.

Fig. 3.3 Energy-band diagram at the surface of a p-type semiconductor. Reproduced from [65] with kind permission from Wiley.

Fig. 3.4 Capacitance-voltage curve for an ideal MIS capacitor with p-type substrate. Adapted from [65] and [66].

Fig. 3.5 Overview of different kind of charge. Adapted from [65] ........................................223

Fig. 3.6 High-frequency CV-curve of a MIS structure with effective trapped oxide charge. Adapted from [66].

Fig. 3.7 Schematic diagram of a MISFET. Adapted from [65].

Fig. 3.8 Different types of field effect transistors (first column) with corresponding electrical symbol (second column), adopted from [66]. Third column $I_{D}$ vs. $V_{G S}$ and IV-curves (fourth column), adopted from [69].

Fig. 3.9 Schematic of MISFET under different conditions (left, reproduced from [65] with kind permission from Wiley) and corresponding IV-curve (right). MISFET operated (a) in the linear region, (b) at the onset of saturation, and (c) beyond saturation.

Fig. 3.10 Schematic diagram of different field effect devices. Adopted from [71] .....................32

Fig. 3.11 (a) Schematic view of a suspended gate FET, and (b) a Lundström-FET. Reproduced from [105] with kind permission from Springer Science and Business Media.

Fig. 3.12 (a) Schematic view, and (b) the equivalent electrical circuit of a floating gate FET. Reproduced from [105] with kind permission from Springer Science and Business Media.

Fig. 3.13 Schematic of the potential energy diagram along the reaction coordinate of a molecule $\mathrm{X}_{2}$ approaching a metal surface. Reproduced from [120] with permission from Wiley-VCH. 
Fig. 3.14 Influence of adsorbates on the work function of an n-type semiconductor. (a) Before adsorption, (b) with surface states, SS, (c) with a dipole layer, DL, and (d) with both SS and DL. Reproduced from [122] with kind permission from Elsevier...... 41

Fig. 3.15 Schematic illustration of the sensing mechanism of a FET gas sensor (a) with a dense catalytic gate, and (b) with a porous gate. Adapted from [68].

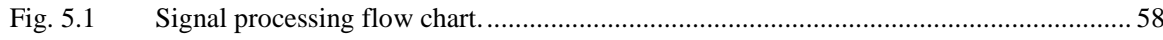

Fig. 5.2 Overview of multivariate pattern analysis techniques. Reproduced from [248] with kind permission from Wiley-VCH.

Fig. 5.3 (a) Scree-plot showing the eigenvalues (blue bars) and cumulative variance (red line) of a PCA. (b) Score plot of the first two principal components discriminating six gases.

Fig. 5.4 (a) Loadings plot of the PCA showing the impact of each feature F. (b) Biplot showing the loadings and the scores in one diagram.

Fig. 5.5 (a) LDA scatter plot showing the discrimination of three classes, (b) territorial plot of a Mahalanobis distance classifier, and (c) territorial plot of a k-Nearest Neighbor classifier with $\mathrm{k}=5$.

Fig. 5.6 LDA scatter plot showing over-fitting. Data set contains 150 observations (cycles) in three groups. (a) Discrimination when 145 features, and (b) only 15 features are used.

Fig. 6.1 Schematic diagram of the gas mixing system used for measurements with typical exhaust gases at the Lab for Measurement Technology.

Fig. 6.2 Schematic diagram of the gas mixing system used for volatile organic compounds measurements. Adapted from [270], [47].

Fig. 6.3 (a) Cross sectional view of a two-terminal depletion type SiC-FET, and (b) picture of the SiC die holding three transistor structures (1-3) and one test structure (4)..........94

Fig. 6.4 Photograph of a SiC-FET mounted on a TO-8 header

Fig. 6.5 (a) Cross sectional view of a two-terminal enhancement type SiC-FET, and (b) picture of the SiC die holding three transistor structures (2-4) and two test structure $(1,5)$

Fig. 6.6 Current-voltage, IV, curve of an enhancement type SiC-FET with iridium gate. 95

Fig. 6.7 (a) Cross sectional view of a three-terminal depletion type SiC-FET, and (b) picture of the $\mathrm{SiC}$ die holding two two-terminal $(1,2)$, two three-terminal transistor

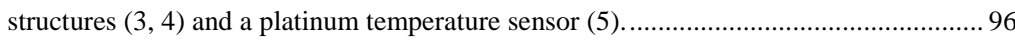

Fig. 6.8 Scanning electron microscopy, SEM, picture of a platinum gate. ................................ 96

Fig. 6.9 Current-voltage, IV, curve of a three-terminal depletion type SiC-FET with platinum gate. Black open symbols mark the response in dry nitrogen with $5 \%$ oxygen and green solid symbols mark the response with additional 500 ppm carbon monoxide. (a) At $100{ }^{\circ} \mathrm{C}$, and (b) at $300{ }^{\circ} \mathrm{C}$.

Fig. 6.10 Exploded view drawing of the sensor chamber. All parts, e.g., sensors, seals, Swagelok fittings, are shown. 
Fig. 6.11 Front panel of the control and data acquisition software tab "general settings". 102

Fig. 6.12 Front panel of the control and data acquisition software tab "temperature cycle".... 102

Fig. 6.13 Front panel of the control and data acquisition software tab "gate / substrate bias cycle".

Fig. 6.14 Front panel of the control and data acquisition software tab "diagrams"..................... 103

Fig. 6.15 Graphical user interface of the LDA-PCA-Tool. .................................................. 106

Fig. 7.1 Schematic explanation of constant drain current operating mode. (a) For a twoterminal device where gate is connected to drain, and (b) for a three terminal device.

Fig. 7.2 Schematic explanation of constant drain-source voltage operating mode. (a) In the linear region, and (b) in saturation region.

Fig. 7.3 Schematic explanation of constant operation mode, respectively constant drain current and constant drain-source voltage by adjusting the gate bias.....

Fig. 7.4 (a) Applied temperature cycle showing the set temperature (red) and actual temperature measured by the additional Pt-100 (brown). Adopted from [283], and (b) dynamic sensor response for $25 \mathrm{ppm} \mathrm{NH}_{3}, 75 \mathrm{ppm} \mathrm{C}_{3} \mathrm{H}_{6}, 100 \mathrm{ppm} \mathrm{NO}$,

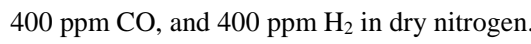

Fig. $7.5 \quad$ (a) Normalized dynamic sensor response when setting the cycle mean to one with feature intervals marked adopted from [283], and (b) difference (normalized) sensor response.

Fig. 7.6 (a) Discrimination of $\mathrm{H}_{2}, \mathrm{CO}, \mathrm{C}_{3} \mathrm{H}_{6}, \mathrm{NO}$, and $\mathrm{NH}_{3}$ in dry nitrogen, and (b) quantification of different concentrations of $\mathrm{NH}_{3}$. The $0 \mathrm{ppm}$ group, i.e., pure nitrogen, has been projected into the scatter plot.

Fig. 7.7 (a) Verification of the TCO approach, and (b) investigation of the stability. Training data are marked by open symbols, projected data by semi-solid symbols. In dry nitrogen.

Fig. 7.8 Applied gate bias sweeps (blue) and corresponding sensor response to $500 \mathrm{ppm}$ ammonia and 500 ppm carbon monoxide in dry synthetic air at three temperatures. Adopted from [286].

Fig. 7.9 Hysteresis curves for $\mathrm{NH}_{3}$ and $\mathrm{CO}$ in dry $\mathrm{N}_{2}$ when sweeping the gate bias from $-3 \mathrm{~V}$ to $+7 \mathrm{~V}$ in $900 \mathrm{~s}$ and back. (a) Absolute signal at $185^{\circ} \mathrm{C}$, (b) difference signal at $185^{\circ} \mathrm{C}$, (c) absolute signal at $265^{\circ} \mathrm{C}$, and (d) difference signal at $265^{\circ} \mathrm{C}$. Adopted from [286].

Fig. 7.10 Difference hysteresis curves for $\mathrm{NH}_{3}$ and $\mathrm{CO}$ at $265^{\circ} \mathrm{C}$ when sweeping the gate bias from $-3 \mathrm{~V}$ to $+7 \mathrm{~V}$ in $900 \mathrm{~s}$ and back again. (a) In dry synthetic air, i.e., $20 \%$ $\mathrm{O}_{2}$ in $\mathrm{N}_{2}$, and (b) in nitrogen with $50 \%$ r.h. at $20{ }^{\circ} \mathrm{C}$. Adopted from [286]

Fig. 7.11 (a) Gate bias cycle, and (b) LDA scatter plot showing the discrimination of $500 \mathrm{ppm} \mathrm{NH}_{3}, 500 \mathrm{ppm} \mathrm{CO}$, and synthetic air under dry condition. Only the hysteresis height, width, and area under curve at both temperatures are used as features. 
Fig. 7.12 LDA scatter plot showing the discrimination of $500 \mathrm{ppm} \mathrm{NH}_{3}, 500 \mathrm{ppm} \mathrm{CO}$, and synthetic air under dry condition. Only the hysteresis height, width, and area under curve at $260{ }^{\circ} \mathrm{C}$ are used as features.

Fig. 7.13 Change of feature value over time for increasing concentration steps. (a) Promising features, and (b) features which represent only noise.

Fig. 7.14 Diagram showing the value of the univariate F-value in descending order for the discrimination shown in Fig. 7.15a.

Fig. 7.15 (a) Discrimination of $\mathrm{H}_{2}, \mathrm{CO}, \mathrm{C}_{3} \mathrm{H}_{6}, \mathrm{NO}$, and $\mathrm{NH}_{3}$, and (b) corresponding loadings plot.

Fig. 7.16 Diagram showing the value of the average coefficient in descending order for the discrimination shown in Fig. 7.15a.

Fig. 7.17 LDA scatter plot showing the discrimination of $\mathrm{H}_{2}, \mathrm{CO}, \mathrm{C}_{3} \mathrm{H}_{6}, \mathrm{NO}$, and $\mathrm{NH}_{3}$ similar to Fig. 7.15a but after feature selection based on (a) the univariate F-value, (b) the average coefficient and the loadings plot, and (c) the stepwise LDA algorithm.

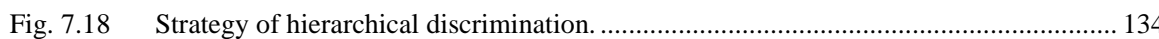

Fig. 7.19 Modified hierarchical strategy for improved stability.

Fig. 8.1 Test gas profile. The ratio between $\mathrm{NO}$ and $\mathrm{NO}_{2}$ is kept constant at 10:1. The concentration range of $\mathrm{NO} / \mathrm{NO}_{2}$ starts at $50 / 5 \mathrm{ppm}$, and increases in $25 / 2.5 \mathrm{ppm}$ steps up to 200/20 ppm. The standard mixture consists of $600 \mathrm{ppm} \mathrm{CO}, 50 \mathrm{ppm}$ $\mathrm{C}_{2} \mathrm{H}_{4}$, and $60 \mathrm{ppm} \mathrm{NH}_{3}$ in an atmosphere of $10 \%$ oxygen in nitrogen.

Fig. 8.2 Difference in the sensor response between a mixture of $600 \mathrm{ppm} \mathrm{CO}, 50 \mathrm{ppm} \mathrm{C}_{2} \mathrm{H}_{4}$, and $60 \mathrm{ppm} \mathrm{NH}_{3}$, and either $100 \mathrm{ppm} \mathrm{NO}$ (yellow triangles) or $10 \mathrm{ppm} \mathrm{NO}_{2}$ (orange diamonds) in a humid atmosphere, i.e., $50 \%$ r.h., of $10 \%$ oxygen in nitrogen. The temperature is increased linearly from $100{ }^{\circ} \mathrm{C}$ up to $400{ }^{\circ} \mathrm{C}$ in $45 \mathrm{~min}$ (red circles). Adopted from [273].

Fig. 8.3 Designed temperature cycle for $\mathrm{NO}_{\mathrm{x}}$ detection (red squares) and sensor signal in pure nitrogen (black triangles). Adopted from [273].

Fig. 8.4 Quasi-static sensor response towards $\mathrm{C}_{2} \mathrm{H}_{4}, \mathrm{CO}, \mathrm{NO}_{2}, \mathrm{NO}$, and $\mathrm{NH}_{3}$ in a humid atmosphere, i.e., $50 \%$ r.h., with $10 \%$ oxygen in nitrogen. Adopted from [273].

Fig. 8.5 Dynamic sensor response of different $\mathrm{NO} / \mathrm{NO}_{2}$ concentrations in a mixture of 600 ppm CO, $50 \mathrm{ppm} \mathrm{C}_{2} \mathrm{H}_{4}$, and $60 \mathrm{ppm} \mathrm{NH}_{3}$ with $50 \%$ r.h. and $10 \% \mathrm{O}_{2}$. (a) Raw signal with an insert showing a detailed view of the response at $187^{\circ} \mathrm{C}$, and (b) difference (normalized) signal. Normalization is done by setting the cycle mean to 1. Nine intervals are marked for feature extraction.

Fig. 8.6 (a) LDA scatter plot showing the discrimination of seven $\mathrm{NO}_{\mathrm{x}}$ concentrations in the standard mixture at $60 \%$ r.h., and (b) second order polynomial fit of the group centroids over their value of DF1.

Fig. 8.7 (a) LDA scatter plot when the LDA is trained with only three $\mathrm{NO}_{\mathrm{x}}$ concentrations, i.e., 50/5, 125/12.5, and 200/20 ppm (solid symbols). "Unknown" concentrations, i.e., $75 / 7.5,100 / 10,150 / 15$, and $175 / 17.5 \mathrm{ppm}$, are projected using the obtained 
LDA coefficients (semi-solid symbols). (b) Fit of the centroids of the training groups over DF1 and projection of "unknown" concentrations.

Fig. 8.8 (a) Regression plot of a PLSR model build by the seven $\mathrm{NO}_{\mathrm{x}}$ concentrations when nine components are used, and (b) when only three concentrations are used for building the model (blue open symbols). Projected "unknown" concentrations are marked with red solid symbols

Fig. 8.9 Hierarchical strategy for $\mathrm{NO}_{\mathrm{x}}$ quantification in a varying background....

Fig. 8.10 (a) LDA scatter plot showing the discrimination of backgrounds. Each group contains four $\mathrm{NO}_{x}$ concentrations, i.e., 0, 50/5, 125/12.5, and 200/20 ppm in two different backgrounds, and (b) territorial plot when a kNN-classifier is used with $\mathrm{k}=5$.

Fig. 8.11 LDA scatter plot showing the discrimination of backgrounds. Projected groups of "unknown" $\mathrm{NO}_{x}$ concentrations, i.e., 50/5, 125/12.5, and 200/20 ppm $\mathrm{NO}_{\mathrm{x}}$ in a background with an increased $\mathrm{NH}_{3}$ concentration, are marked with semi-solid symbols.

Fig. 8.12 LDA scatter plot showing the discrimination of four $\mathrm{NO}_{\mathrm{x}}$ concentrations (open symbols) in a background mixture with deviating two $\mathrm{NH}_{3}$ concentrations, i.e., $25 \mathrm{ppm}$ and $100 \mathrm{ppm}$, from the standard mixture. Projection of groups of "unknown" $\mathrm{NO}_{\mathrm{x}}$ concentrations, i.e., 50/5, 125/12.5, and 200/20 ppm in 75 ppm $\mathrm{NH}_{3}$ are marked with semi-solid symbols.

Fig. 8.13 Applied temperature cycles with set and actual temperatures as well as the sensor signal in synthetic air. (a) Platinum-gate SiC-FET, and (b) iridium-gate SiC-FET

Fig. 8.14 Relative sensor response for naphthalene at two humidity levels and in a mixture with ethanol. (a) Platinum-gate SiC-FET, adapted from [47], and (b) iridium-gate SiC-FET.

Fig. 8.15 (a) Relative sensor response for formaldehyde at two humidity levels and at two temperatures, and (b) sensor response for benzene. Reproduced from [47] with kind permission from IEEE.

Fig. 8.16 Normalized difference signal of a Pt-gate SiC-FET for $100 \mathrm{ppb}$ formaldehyde, $20 \mathrm{ppb}$ naphthalene, and $4.5 \mathrm{ppb}$ benzene with $40 \%$ r.h. at $20^{\circ} \mathrm{C}$. Nine intervals for feature extraction are marked. Adapted from [299].

Fig. 8.17 LDA score plot showing the discrimination of several concentrations of formaldehyde, naphthalene, and benzene with $40 \%$ r.h. when a Pt-gate SiC-FET is used.

Fig. 8.18 (a) LDA scatter plot showing the discrimination of formaldehyde, naphthalene, benzene, and the "below threshold" group when a Pt-gate SiC-FET is used. (b) Territorial plot of the discrimination when a Mahalanobis distance classifier is used. Adopted from [299].

Fig. 8.19 Schematic of a two layer decision process for quantification of VOC 
Fig. 8.20 PLS-DA scatter plots showing the separation of formaldehyde, naphthalene, benzene, and the "below threshold" group with combined humidity levels, i.e., $20 \%$ and $40 \%$ r.h. 10 components are used for the classification. Adopted from [299].

Fig. 8.21 LDA scatter plot showing the discrimination of different concentrations of formaldehyde. (a) With $40 \%$ r.h., and (b) with two combined levels of relative humidity, i.e., $20 \%$ and $40 \%$ r.h.

Fig. 8.22 LDA scatter plot showing the discrimination of different concentrations of naphthalene at $20 \%$ r.h. and the influence of humidity. First, different concentrations at $20 \%$ r.h. are discriminated (open symbols). Based on this, data at $40 \%$ r.h. are projected (semi-solid symbols). Adopted from [47].

Fig. 8.23 Top: LDA scatter plot showing the discrimination of different concentrations of naphthalene independent of the humidity level. Bottom: histogram showing the distribution of the scores, i.e., cycles, along DF1. Adopted from [298], [299]...

Fig. 8.24 Top: Quantification of naphthalene. Training data are marked with open symbols, projected unknown data are marked by semi-solid symbols. Bottom: Plot concentration vs. DF1 value with second order fit of the training data. The projected data are marked by semi-solid symbols. A cutting line is given which can be used as a threshold, e.g., for ventilation control. Adopted from [298], [299].

Fig. 8.25 Result of PLSR for quantification of naphthalene independent of the humidity. (a) When all concentrations are used to build the model, and (b) when only three concentrations are used. Training data are marked by blue open symbols while projected data are marked by red semi-solid symbols. Adopted from [299].

Fig. 8.26 (a) LDA scatter plot showing the discrimination of formaldehyde, naphthalene, and benzene with four concentrations each in three different ethanol backgrounds, and (b) discrimination of the three ethanol backgrounds. The level of r.h. is $20 \%$.

Fig. 8.27 (a) LDA scatter plot showing the discrimination of formaldehyde, naphthalene, and benzene with four concentrations each in $0.5 \mathrm{ppm}$ ethanol, and (b) discrimination of formaldehyde and naphthalene in $1 \mathrm{ppm}$ ethanol. The level of r.h. is $20 \%$.

Fig. 8.28 (a) LDA scatter plot showing the discrimination of different naphthalene concentrations in three different ethanol backgrounds, and (b) discrimination of different formaldehyde concentrations. The level of r.h. is $20 \%$.

Fig. 8.29 LDA scatter plot showing the discrimination of different naphthalene concentrations. Each group contains data with two different background ethanol concentrations, i.e., $0.5 \mathrm{ppm}$ and $1 \mathrm{ppm}$. The level of r.h. is $20 \%$.

Fig. 8.30 (a) Combination of temperature cycle (part A and C) consisting of two plateaus and gate bias cycle combining plateaus and ramps (parts B and D), (b) dynamic sensor response for $800 \mathrm{ppm} \mathrm{CO}, 50 \mathrm{ppm} \mathrm{\textrm {NO } _ { 2 }}$, and $75 \mathrm{ppm} \mathrm{NH}_{3}$ in $\mathrm{N}_{2}$ with $5 \% \mathrm{O}_{2}$, and (c) standardized dynamic sensor response with marked feature intervals. Adopted from [219] 
Fig. 8.31 LDA scatter plots showing the quantification of carbon monoxide when using features from (a) only the temperature change, i.e., parts A and C, (b) only from the bias cycle at $200{ }^{\circ} \mathrm{C}$, i.e., part $\mathrm{D}$, and (c) the temperature change and both gate bias cycles. Adopted from [219].

Fig. 8.32 Exemplary features (here: mean values, mv) and their change over time due to exposure to different test gases and different concentrations. The carrier gas is $5 \%$ oxygen in nitrogen. Reproduced from [219] with kind permission from Elsevier.

Fig. 8.33 (a) LDA showing the discrimination of $\mathrm{CO}, \mathrm{NO}_{2}$, and $\mathrm{NH}_{3}$ with four concentrations each as well as the leave-one-out cross-validation rate, $\mathrm{CV}$, the multivariate Wilks'

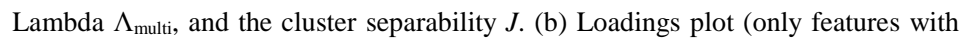
an average coefficient greater than 0.1 are shown), mv: mean value, sec: secant. Reproduced from [219] with kind permission from Elsevier.

Fig. 8.34 Diagram showing the value of the average coefficient for the 18 most significant features, i.e., features with an average coefficient greater than 0.1 .

Fig. 8.35 (a) LDA showing the discrimination of $\mathrm{CO}, \mathrm{NO}_{2}$, and $\mathrm{NH}_{3}$ with four concentrations each when only features with an average coefficient greater than 0.1 were used as well as the leave-one-out cross-validation rate, CV, the multivariate Wilks' Lambda $\Lambda_{\text {multi }}$ and the cluster separability $J$. (b) Feature selection based on the loadings plot. In total eight features were used

Fig. 8.36 Detailed view of the discrimination shown in Fig. 8.35b. (a) For carbon monoxide, (b) for ammonia, and (c) for nitrogen dioxide.

Fig. 8.37 PCA scatter plot showing the discrimination of $\mathrm{CO}, \mathrm{NO}_{2}$, and $\mathrm{NH}_{3}$ with four concentrations each when the sensor data, i.e., all 900 data points per cycle, is used as input for calculating the PCA

Fig. 8.38 (a) LDA scatter plot showing the discrimination of $\mathrm{CO}, \mathrm{NO}_{2}$, and $\mathrm{NH}_{3}$ with four concentrations each when the first 36 principal components are used as features. The PCA is calculated based on sensor data. (b) Corresponding loadings plot. Only features with an average coefficient greater than 0.8 are shown.

Fig. 8.39 (a) Scatter plot of a standardized PCA showing the discrimination of $\mathrm{CO}, \mathrm{NO}_{2}$, and $\mathrm{NH}_{3}$ with four concentrations each when all extracted features are used, and (b) corresponding loadings plot.

Fig. 8.40 (a) LDA scatter plot showing the discrimination of $\mathrm{CO}, \mathrm{NO}_{2}$, and $\mathrm{NH}_{3}$ with four concentrations each when the first 36 principal components are used as features. The PCA was calculated based on extracted features. (b) Corresponding loadings plot. Only features with an average coefficient greater than 0.8 are shown.

Fig. 8.41 LDA scatter plots showing the discrimination of $\mathrm{CO}, \mathrm{NO}_{2}$, and $\mathrm{NH}_{3}$. Each group consists of four concentrations. Training data are marked by open symbols and test data of a second measurement performed five days later by semi-solid symbols. The overall correct classification rate is obtained by a Mahalanobis distance 
classifier. (a) Raw data, (b) normalization by setting cycle mean to 1 , and (c) standardized cycle, i.e., setting cycle mean to 0 and standard deviation to 1 .

Fig. 8.42 LDA scatter plot showing the discrimination of $\mathrm{CO}, \mathrm{NO}_{2}$, and $\mathrm{NH}_{3}$ independent of concentration when the data are standardized first. Training data are combined of two measurements and marked by open symbols. Test data of a third measurement performed five days after the training are marked with semi-solid symbols and are classified by a Mahalanobis distance classifier.

Fig. 9.1 Sulfur dioxide concentration in a pilot plant measurement. The signal from the reference instrument (black solid line) is compared to the sensor signal processed by using exponential fit from a LDA model (red dotted line). Reproduced from [97] with kind permission from Elsevier. 


\section{Tables}

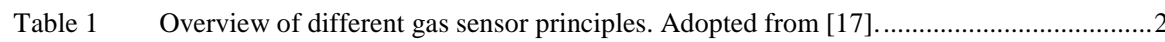

Table 2 Indoor air pollutants of interest with highest relevance and current exposure limit.

Reproduced from [47] with kind permission from IEEE.

Table 3 Result of leave-one-out cross-validation with either Mahalanobis distance or kNearest Neighbor, i.e., $\mathrm{k}=3$, classifier for LDA shown in Fig. 5.6.

Table $4 \quad$ Requirements for control and read-out hardware .........................................................99

Table 5 Overview of software tools at the Lab for Measurement Technology. ............................105

Table 6 Overview of feature selection methods and their suitability for automatization.............131

Table $7 \quad$ Overview of different background mixtures in the extended gas profile. .......................139

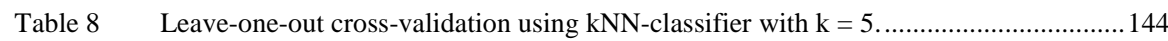

Table 9 Correct classification rate obtained by a $\mathrm{kNN}$-classifier with $\mathrm{k}=5 \ldots \ldots \ldots \ldots \ldots \ldots \ldots \ldots \ldots \ldots . . .149$

Table 10 Leave-one-out cross-validation of the discrimination shown in Fig. 8.18a using a Mahalanobis distance classifier.

Table 11 Leave-one-out cross-validation of the discrimination of different naphthalene concentrations in ethanol, cf. Fig. 8.29, using a Mahalanobis distance classifier. ..........171

Table 12 Parameters describing the quality of the discrimination shown in Fig. 8.31_.................177

Table 13 Features selected by the average coefficient and the loadings plot...............................181 



\section{Acknowledgement}

As a PhD student at two universities, in two different countries, I had the pleasure to meet with many admirable and good-hearted people with whom I am sharing splendid experiences. Without out those people, a scientific thesis like this would just be a summary of the obtained results written on a piece of paper but the last four years are more than this. In fact, the entire project would not have been possible without the help and support of excellent supervisors guiding me, pleasant colleagues always having a sympathetic ear and time to discuss not only about science, and friends and family being there whenever I needed them. Even though, time was not always as easy as it seems, I really enjoyed being a $\mathrm{PhD}$ student and I am thankful for all the experiences I gained. On these last pages, I will take the opportunity to honor all those that have been accompanying me throughout this part of my life.

First of all, I would like to show my appreciation to Prof. Anita Lloyd Spetz and Prof. Andreas Schütze, my outstanding supervisors. I am really glad that I could continue working with both of you and that we managed to do this joint $\mathrm{PhD}$ project together. It was in August 2004 when I started in Andreas' group, at that time as a school student, and worked on the famous Güterbahnhof - my first project with gas sensors. I would like to thank Andreas for this unforgettable, faithful, and successful time (it is more than ten years by now) as well as his support and trust. I also would like to thank Annette and Andreas for their hospitality and organizing the annual garden parties. Then, in autumn 2009 during my ERASMUS exchange, I met Anita. Who would have thought at that time that this was the starting point of such a strong collaboration between both groups? Anita was more than just a supervisor and scientific role model. She is a very warm-hearted person always in a good mood. Even outside the university she helped me a lot and tried her best making me feel like home during my stays in Linköping and Oulu. I am grateful that both Anita and Andreas gave me the opportunity to do this joint $\mathrm{PhD}$. They always supported me and 
gave me the room to work very independently as well as given me the chance to present my work - all over the world - at many international conferences.

First and foremost, I would not have succeeded without the help of my dear friend and supervisor Mike Andersson. Everything has started in autumn 2009 with a small sensor project for a random ERASMUS student. At this time, it never would have come to my mind that based on a project work we would become really good friends. Not only in work related questions, he helped and supported me a lot but also outside university business it was always a pleasure meeting with him. Be it playing innebandy, the dinners at his place, the evenings in town, or all the other things we did together, like the trip to Kuusamo or watching the ice hockey World Championship in Stockholm. He is truly the best supervisor one can wish for and I would like to thank you very much. I am sure we will stay in close contact also after my time as a PhD student no matter where we are going in the future. I promise I will join the legendary ski and hiking trip at least once. At this point, I would like to thank also Mille; I always had fun when she was visiting Linköping. I am looking forward meeting both of you soon.

I would like to thank all my colleagues from the Lab for Measurement Technology for being there and creating a friendly and enjoyable working atmosphere. Not to mention the hiking trips to the Kleinwalsertal and other free time activities we did together. First of all, I would like to show my appreciation to the former $\mathrm{PhD}$ students Peter Reimann, Stephan Horras, René Sallier, Karsten Kühn, and Torsten Bley, who I got to know already during my studies as good mentors, and later on as superb colleagues, too. I am glad that Thomas Fricke returned to the lab after a few years 'break'. It was very good to have someone talking to about data processing but also other things not only work related. Thank you. Also my fellow PhD students Eliseo Pignanelli, Marco Schüler, Bastian Schmitt, Nikolai Helwig, Martin Leidinger, and Marvin Schmidt I would like to thank for the fabulous and inspiring time at work but also outside the university. Especially, I would like to honor Manuel Bastuck who started as a bachelor student of mine and being now a fellow $\mathrm{PhD}$ student. He contributed a lot to my research and it was always good to have someone listening and talking to about field effect devices, Sweden, and weird Swedish traditions, and other random things. I am happy that I managed to bring him to Linköping and that he continues now with a joint $\mathrm{PhD}$, too. I wish you as many 
magnificent experiences as I had as a joint $\mathrm{PhD}$ student and good luck. Besides my fellow PhD students, I would like to thank, particularly, Harald Nagel who keeps the group together and has always a ready ear. I remember very well the first days when I started as a school student and shared the office with him and Anke Kopper. It never would have come to my mind that working at the university is so much fun. Probably, it was one of these moments when I realized that I want to continue at university and, in particular, in this group. I would like to thank also Siv Hocke who is Swedish and tried her best to teach me. It was great to celebrate Swedish traditions also in the group at Saarland University, like having semlor, kanelbullar, or lussekatter. I also would like to thank all the others, Tilman Sauerwald, André Kreiter, the diploma workers, and student assistants I had the fortune to supervise and work together with: Tizian Schneider who introduced Support Vector Machines to our group, Gina Monzon, Marius Rodner, Tobias Baur, Michael Schirrman, Ralf Jung, Nicolas Jäckel, Henrik Lensch, Caroline Schultealbert and everyone I might have forgotten. It makes me feel good to see that the next generation of possible $\mathrm{PhD}$ students and future scientists is ready to continue research at LMT.

I was privileged to work in more than one group as a $\mathrm{PhD}$ student. Therefore, I would like to honor all my friends and colleagues at IFM for making my visits always fabulous and enjoyable. The Division of Applied Sensor Science is more like a big family and even though, I spent only short periods of time in Linköping, I always felt very welcome. Above all, I would like to thank Donatella Puglisi and Jens Eriksson for their hospitality and their efforts for the whole group. I also enjoyed the time when you were visiting Saarbrücken and I hope it was not the last time that we could welcome you. I would like to acknowledge also my fellow $\mathrm{PhD}$ students, Zhafira Darmastuti who used the methodology I suggested in this thesis and showed that it works also in the field, Hossein Fashandi, always very kind, Lida Khajavizadeh, and of course Peter Möller. Especially, I would like to acknowledge Peter, also part time lab engineer, for all the help in the lab and the 'quick and dirty' solution during my visits so that I could start / continue measuring. I also remember the time together in Oulu and other occasions in Linköping. I hope you will have such a fantastic time as I had being a PhD student. Not to forget, I thank Robert 'Bob' Björklund for making me laugh in the lab even if work was frustrating. 
I would like to take the opportunity to show my appreciation to Therese Dannetun for helping me with administration and travel arrangements and Stefan Klintström for his dedication to the scientific area and taking care of me and all the other $\mathrm{PhD}$ students. I also would like to thank 'my' innebandy team at the university. Thank you very much for the chance to practice and play with you. It is a pleasure to play with you guys. As representatives, I would like to mention Prof. Hans Arwin and Prof. Kenneth Järrendahl.

First of all, I would like to thank Cristina and Ove Sollie and the always friendly and anxious staff at cozy Hotell du Nord. I really love staying with you - it feels like home to me. I hope seeing you soon again and maybe you will manage at some point to leave the hotel alone for a week or two and come to visit Saarbrücken, again. You are always very welcome.

First of all, I would like to show my appreciation to Prof. Frank Mücklich and Flavio Soldera the managers of The Joint European Doctoral Programme in Materials Science and Engineering - DocMASE, who kindly agreed that I can participate in this valuable program. Not to forget, I thank all my fellow DocMASE students, especially those, who are also working either at Linköping University or at Saarland University. Despite, there are many issues to cope with, coming along with working in two different countries, I think we had a good time together especially, during the annual summer schools. Although, I have to admit that I have not entirely understood what your research projects are about. Good luck everyone! Furthermore, I would like to acknowledge those, who helped with the organization of the different training schools, as well as being in charge for DocMASE at each university. As a representative, I would like to thank Prof. Magnus Odén at Linköping University.

All my work would not have been possible without the support of the two Spin-off companies, 3S - Sensors, Signal Processing, Systems GmbH and SenSiC AB. First of all, I would like to thank Thorsten Conrad 'TC' and Wolfhard Reimringer from 3S. Especially, I would like to honor Wolfhard who had to struggle with the FET platforms many times and he gave his best to provide a good hardware board so that I can perform my measurements. Thanks a lot. Additionally, I would like to thank Ulf 'the wolf' Thole and Lars Hammarlund from SenSiC for providing the SiC-FET sensors. I also liked meeting you in Linköping and the chatting during lunch breaks or in the lab. 
I was not only involved in the research school DocMASE but also in the meetings of PhD students working in the field of gas sensing in Germany. It was always great meeting the other $\mathrm{PhD}$ students from the different groups, and each time was legendary. As representatives, I would like to mention Isabella Marr, Jens Langejürgen, Philipp Cochems, and Dominik Klaus.

I would like to acknowledge my colleagues at the University of Oulu. I learned that Oulu is located in the middle of Finland and not, as I used to say, in the North but for me it is pretty much north anyway. First of all, I would like to thank Prof. Jyrki Lappalainen, Joni Huotari, Nina Halonen, and Maciej Sobocinski, for the great times in Oulu. Not to forget, I would like to thank Liisa Kangasharju for helping me with the administration, Antti Paavola for technical help, and Antti Uusimäki for introducing me to pesäpallo (Finnish Baseball).

All the work would not have been possible without the support of my good old friends. First of all, I would like to honor Stefan Hartmann, Daniel Hartmann, and Christoph Schweizer. Especially, I would like to thank Christoph for proof reading this thesis. I know it was not easy to read through all this gas sensor stuff but I am happy you did and that you gave valuable comments.

I also would like to say thank you and, at the same time, say sorry to my soccer team SV St. Ingbert who had to play several times without me - their goalkeeper - when I was again on travel.

Last but not least, I am very grateful to my parents, Helmut and Irene, and my brother Sebastian supporting me during my whole studies and the $\mathrm{PhD}$. It was not always guns and roses but with the best support one can wish for I completed this chapter of my life successfully. Especially, I would like to honor my beloved Anna for always supporting me and given me the energy to complete the thesis. Thank you also for proof-reading the thesis. I am looking forward for the next period of our life.

And, I would like to thank all those that should be mentioned here but due to technical issue are not. 



\section{Affidavit /}

\section{Eidesstattliche Versicherung}

I hereby affirm that I wrote this thesis on my own and without assistance from third parties. I used no other aids than the listed and specified sources and resources.

Saarbrücken, February 2, 2015

(Christian Bur)

Hiermit versichere ich an Eides statt, dass ich die vorliegende Arbeit selbstständig und ohne Benutzung anderer als der angegebenen Hilfsmittel angefertigt habe. Die aus anderen Quellen oder indirekt übernommenen Daten und Konzepte sind unter Angabe der Quelle gekennzeichnet. Die Arbeit wurde bisher weder im In- noch im Ausland in gleicher oder ähnlicher Form in einem Verfahren zur Erlangung eines akademischen Grades vorgelegt.

Saarbrücken, 02. Februar 2015

(Christian Bur) 

